\title{
Electrophysiological Analysis of the Synaptic Vesicle Priming Process
}

\author{
Dissertation \\ for the award of the degree \\ "Doctor rerum naturalium" \\ of the Georg-August-Universität Göttingen \\ within the doctoral program Neurosciences \\ of the Georg-August University School of Science (GAUSS)
}

submitted by

Dennis Bernd Nestvogel

born in

Fritzlar, Germany

Göttingen, 2017 

I hereby declare that this thesis was written independently and with no other sources and aids than quoted.

Dennis Nestvogel

Göttingen, $31^{\text {st }}$ of March, 2017 


\section{Table of Content}

Acknowledgements

Abstract

List of Abbreviations

List of Figures

1. Introduction

1.1. Basic Principles of Chemical Synaptic Transmission.......................................................... 6

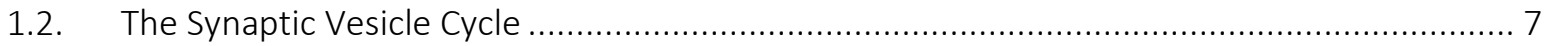

1.2.1. Synaptic Vesicle Fusion is Catalyzed by Members of the SNARE Protein Family ............. 8

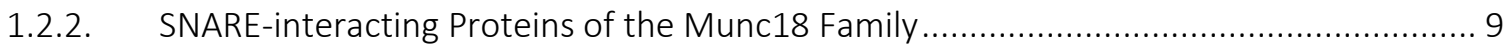

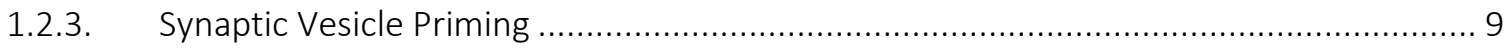

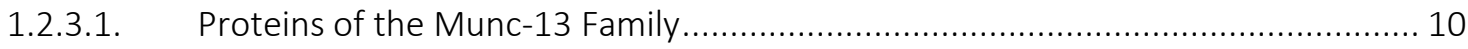

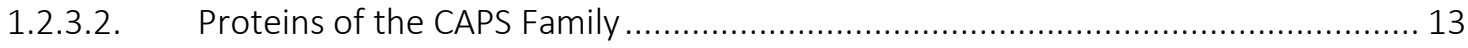

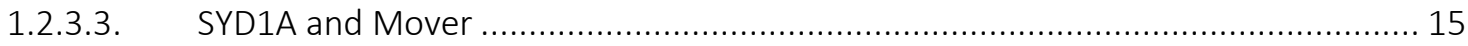

1.3. Relevance of SV priming for synaptic plasticity .......................................................... 15

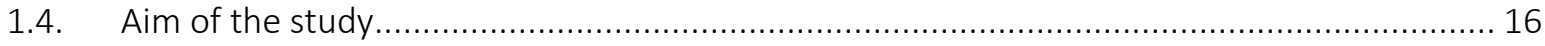

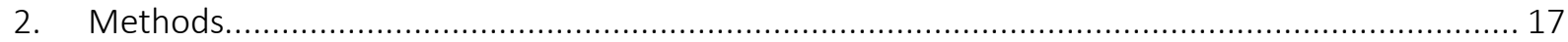

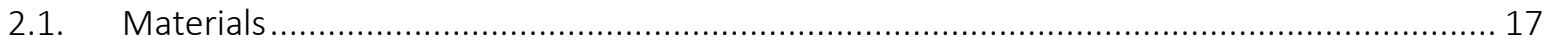

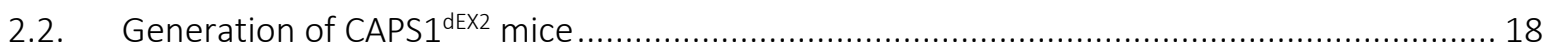

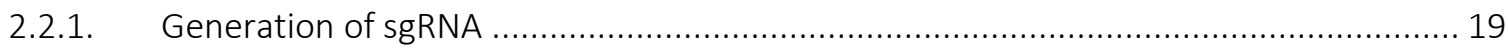

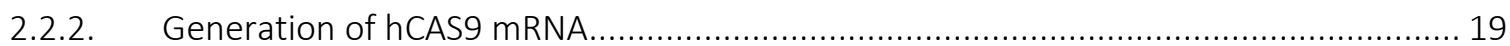

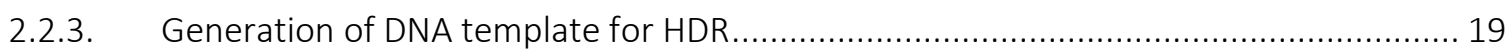

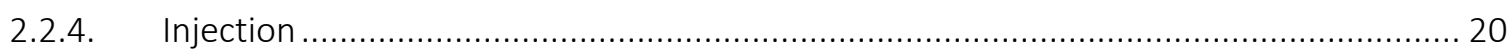

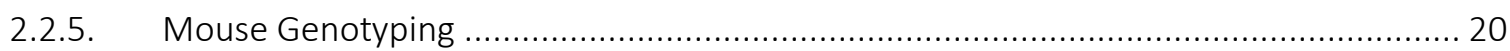

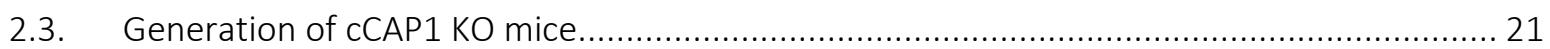

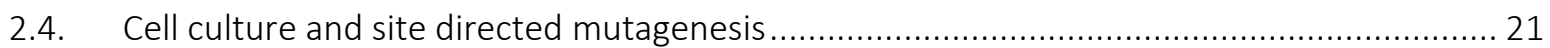

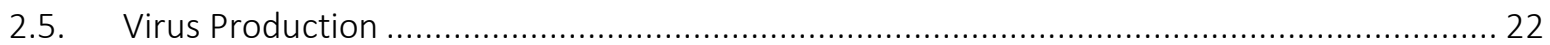

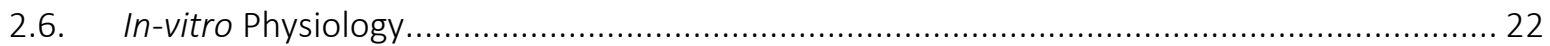

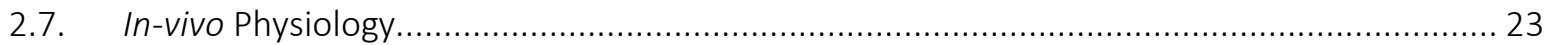

2.7.1. Mouse surgery and in-vivo blind patch clamp recordings......................................... 24

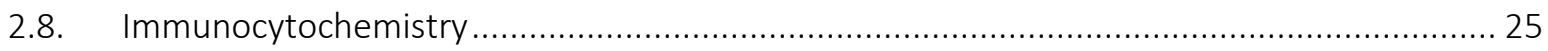

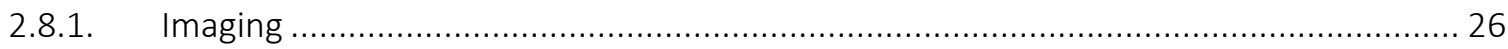

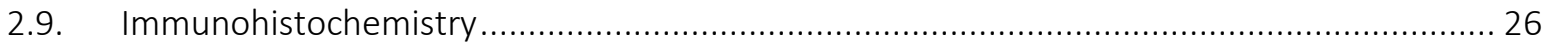


3. Results

3.1. Electrophysiological analysis of the relevance of mSYD1s and Mover in regulating transmitter release in cultured neurons..... . 28

3.1.1. Synaptic transmission is not perturbed in cultured mSYD1A-deficient neurons........... 28

3.1.2. Synaptic transmission is not altered in cultured mSYD1A,B DKO neurons.................. 31

3.1.3. Deletion of Mover does not lead to changes in synaptic transmission in cultured hippocampal neurons.

3.2. Electrophysiological dissection of the functional interplay between CAPSs and Munc13s

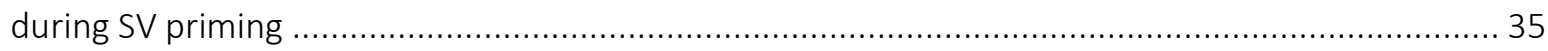

3.2.1. PDBu partially rescues the priming deficit in CAPS DKO neurons .............................. 35

3.2.2. The $\mathrm{C} 1$ domain of Munc13 is required to functionally compensate for the loss of CAPSs

3.2.3. Electrophysiological dissection of the structure function relationship of CAPSs during SV priming

3.2.3.1. The SNARE-binding MUN domain and the DCV binding site of CAPSs are not essential for SV priming

3.2.3.2. The central PH domain of CAPSs is essential for basic transmitter release

3.2.3.3. Deletion of a conserved protein region within the DI-domain of CAPSs does not lead to changes in transmitter release

3.2.3.4. Overexpression of CAPS2e is sufficient to sustain AP-evoked release in CAKI cells 54

3.3. The relevance of CADPS1 for early-onset bipolar disorder 57

3.3.1. The S399L mutation within the C2 domain of CADPS1 leads to less pronounced shortterm depression

3.3.2. Exon 2 deletion in CADPS1 leads to enhanced short-term synaptic depression......

3.4. Development of an experimental paradigm for the study of SV priming factors in the context of neural circuit function

3.4.1. Targeting the deletion of presynaptic proteins to thalamic nuclei 64

3.4.2. In-vivo whole cell recordings allow the monitoring of sensory evoked subthreshold responses

4. Discussion 70

4.1. Overview 70

4.2. Proteins of the mSYD1 family are not involved in modulating transmitter release in cultured autaptic neurons.

4.3. The presynaptic protein Mover does not regulate release probability in cultured autaptic

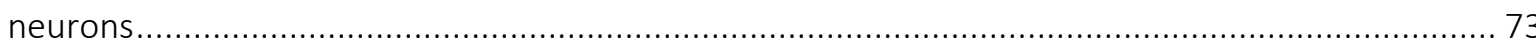

4.4. SV priming in absence of CAPS proteins requires the C1 domain of Munc13.........

4.5. Analysis of the structure function relationship of CAPS proteins reveals key insights into the SV priming process

4.5.1. The C-terminal half of CAPS is not essential to sustain basic synaptic transmission ...... 77 
4.5.2. SV Priming requires the $\mathrm{PIP}_{2}$ binding $\mathrm{PH}$ domain of CAPSS

4.5.3. Deletion of a conserved stretch of amino acids within the DI domain of CAPS2e does not perturb synaptic transmission

4.5.4. BPD associated mutations in CADPS1 change the degree of STD in cultured neurons.. 80

4.6. A hypothetical model of the synaptic vesicle priming process 82

4.7. Future prospects in studying the relevance of presynaptic computation in the context of sensory processing......

4.7.1. Thalamic deletion of presynaptic proteins 85

4.7.2. In-vivo patch clamp recordings allow the monitoring of sensory evoked subthreshold responses.

5. Summary. .86

6. Supplementary. 99

CV. 108 


\section{Acknowledgements}

I would like to express my deepest gratitude to my supervisor Dr. JeongSeop Rhee, who has continuously supported me throughout my PhD studies. I really enjoyed our stimulating discussions and I am thankful for the many lessons that he taught me in science.

In addition, I wish to thank Dr. Nils Brose for his personal support and for his critical feedback on many of the experiments that I performed.

I am thankful for the additional advice that I received for my PhD work from Dr. Erwin Neher and Dr. Reinhard Jahn during and outside of the committee meetings.

I wish to thank Drs. Stephane Jamain, Jens Rettig, Corinna Wentzel and Julio Viotti for fruitful collaborations and I particularly appreciated their constant enthusiasm for science.

Furthermore, I would like to thank Drs. Michael Brecht, Andrea Burgalossi, Patricia Preston, Mickey London and Massimo Scanziani for helping me to establish in-vivo patch clamp recordings in the visual cortex of mice in my host department.

I am grateful for the support that I received from Fritz Benseler during my PhD studies. He significantly contributed to the successful generation of the CAPS1 ${ }^{\mathrm{dEX} 2}$ mouse line and I learned much from him about various methodologies in the field of molecular biology.

Much support came also from the technical assistance provided by Anja Günther, Ines Beulshausen and Sabine Bolte. In addition, I wish to thank Ivonne Thanhäuser, Christiane Harenberg and Dayana Warnecke for the genotyping of mice and DNA Sequencing.

I had the chance of working in an environment with many friendly colleagues. I particularly thank Noa Lipstein for helpful discussions and for proofreading parts of this thesis. Additionally, I am thankful for critical feedback an various aspects of this work that I received from Dilja Krueger-Burg, Ben Cooper, Cordelia Imig, Silvia Ripamonti, Carolina Thomas, Ali Shaib, Albrecht Sigler, Bekir Altas, Hiroshi Kawabe, Matthieu Hammer, Olga Babaev, Ricardo Merino and Rafael Brune.

Further, I am thankful for the personal support that I received from the coordination office of the Neuroscience PhD program, particularly from Sandra Drube and Dr. Michael Hörner.

Special thanks also to the Boehringer Ingelheim Fonds that provided me with a PhD scholarship and enabled me to attend international conferences.

Last, but not least, I wish to thank my family. Words cannot describe the deep gratitude that I feel for my parents, siblings, my wife and our daughter. Thank you so much for all your love and support throughout the years 


\section{Abstract}

Chemical synaptic transmission is the most prominent communication channel employed by neural circuits. It is initiated by the action potential triggered fusion of transmitter-filled synaptic vesicles with the presynaptic plasma membrane. Proteins of the CAPS and Munc13 families are required to prime synaptic vesicles for fusion before these can release their contents into the synaptic cleft. In the present study, I dissected the functional interplay of both protein families during the priming process and furthermore tested the significance of two recently described presynaptic proteins in the modulation of the Munc13- and CAPS dependent priming reaction. The data presented here indicate that CAPSS and Munc13s differentially prime SVs at low versus high levels of presynaptic activity in a lipiddependent manner. Specifically, CAPSs engage in a lipid-sensitive priming step at low intraterminal $\mathrm{Ca}^{2+}$ concentrations, while Munc13-lipid interactions predominate at high $\mathrm{Ca}^{2+}$ levels. This step, in turn, likely precedes the partial assembly of fusogenic SNARE complexes, which is catalyzed by the MUN domain of Munc13s. The two recently described presynaptic proteins, mSYD1A and Mover, did not modulate the priming reactions as assessed in the autaptic hippocampal culture system.

In addition, I studied the relevance of the priming process in the etiology of bipolar disorder, which involved a detailed structure function analysis of disease associated CADPS1 mutations in cultured hippocampal neurons and the generation of a knock-in mouse carrying one such mutation. Two out of eight mutations led to altered forms of short-term synaptic plasticity in cultured neurons, which may indicate that perturbations of the priming process contribute to the etiology of bipolar disorder.

Lastly, I established an experimental system for the future study of the significance of the priming process for short-term synaptic plasticity phenomena in the context of visual information processing invivo. 


\section{List of Abbreviations}

$\begin{array}{ll}\text { AP } & \text { Action potential } \\ \text { BPD } & \text { Bipolar Disorder } \\ \text { DAG } & \text { Diacyl glyerol } \\ \text { DCV } & \text { Dense core vesicle } \\ \text { EPSC } & \text { Excitatory postsynaptic current } \\ \text { HFS } & \text { High frequency stimulation } \\ \text { PH } & \text { pleckstrin homology } \\ \text { PdBu } & \text { phorbol 12,13-dibutyrate } \\ \text { PIP } 2 & \text { Phosphatidylinositol-4,5bisphosphate } \\ \text { PPR } & \text { Paired pulse Ratio } \\ \text { RRP } & \text { Readily Releasable Pool } \\ \text { STD } & \text { Short-term depression } \\ \text { STP } & \text { Short-term plasticity } \\ \text { SV } & \text { Synaptic vesicle } \\ \text { VDCC } & \text { Voltage dependent calcium channel }\end{array}$




\section{List of Figures}

Figure 1: Domain structure of Munc13-1

Figure 2: Hypothetical model of the Munc13 dependent priming process.

Figure 3: Summary of genetic manipulation.

Figure 4: Synaptic transmission is not altered in cultured mSYD1A-deficient neurons.

Figure 5: Synaptic transmission is not altered in cultured mSYD1-A,B DKO neurons

Figure 6: Synaptic transmission is not altered in cultured neurons from Mover KO mice

Figure 7: PDBu partially rescues transmitter release in CAPS deficient neurons.

Figure 8: Morphological analysis of cultured CAKI neurons

Figure 9: Transmitter release is severely perturbed in cultured CAKI neurons

Figure 10: Expression and synaptic localization of Munc13 $3^{\mathrm{H} 567 \mathrm{~K}}$ is not perturbed in CAKI cells.

Figure 11: Transmitter release can neither be rescued by PDBu, nor by trains of APs in CAKI cells in contrast to CAPS DKO neurons.

Figure 12: Domain structure of CAPS2 splice variants.

Figure 13: The C-terminal half of CAPS2 is not essential for basic synaptic transmission.

Figure 14: Short-term synaptic transmission is altered upon deletion of the C-terminal half of CAPS2.

Figure 15: SV priming is partially impaired in CAPS2e expressing neurons at basic levels of presynaptic activity.

Figure 16: CAPS dependent priming requires a functional PH domain.

Figure 17: CAPS dependent priming requires a $\mathrm{PIP}_{2}$ binding site within the $\mathrm{PH}$ Domain.

Figure 18: A highly conserved stretch of amino acids within the DI-domain of CAPS is not essential for SV priming.

Figure 19: Overexpression of MUN domain lacking CAPS2e is sufficient to rescue basic transmitter release in CAKI neurons.

Figure 20: Domain structure of human CADPS1 splice variant 1.

Figure 21: Cultured neurons expressing CADPS-1 $1^{\text {S399L }}$ exhibit less short-term synaptic depression when compared to controls.

Figure 22: The S399L mutation in CADPS1 leads to a higher RRP replenishment during HFS.

Figure 23: Cultured neurons expressing CADPS-1 ${ }^{\mathrm{dEX} 2}$ exhibit more pronounced short-term synaptic depression when compared to controls.

Figure 24: Sequence results of Exon2 deletion in the mouse CAPS1 gene

Figure 25: SERTcre driven expression of YFP-tagged channelrhodopsin. 
Figure 26: In-vivo whole-cell recording from morphologically identified neuron.

Figure 27: Light-evoked responses in a layer 4 neuron of the mouse visual cortex.

Figure 28: Basic electrophysiological analysis of Layer 4 neurons from the visual cortex of CAPS1 ${ }^{\mathrm{fl} / \mathrm{wt}}$, SERTcre mice.

Figure 29: Basic electrophysiological analysis of a Layer 4 neuron from the visual cortex of a CAPS1 $1^{\mathrm{flff}}$, SERTcre mouse

Figure 30: Hypothetical model of the SV priming process. 


\section{Introduction}

Neural circuits serve as relay stations and integration centers for the processing of environmental stimuli and for the orchestration of motor outputs. The structural and functional building blocks of such circuits are nerve cells, or neurons. These receive and transmit electrical signals along their dendritic and axonic processes, respectively, and convey information from one cell to another at specialized contact sites, called synapses.

Information transfer by neurons is either accomplished electrically, via gap junctions, or chemically involving the action of transmitter molecules (Connors and Long, 2004; Sudhof, 2012a). With few exceptions, chemical synaptic transmission is the most prominent communication channel employed by neural circuits. It is initiated upon the arrival of an electrical stimulus at the axon terminal that tightly couples to the release of transmitters and culminates in the detection and translation of these signals by postsynaptic receptors into graded, postsynaptic currents (Wojcik and Brose, 2007). The amplitude of such currents is a measure for the synaptic strength between the sending and the receiving cell that can change in an activity- and time-dependent manner (Del Castillo and Katz, 1954). Changes lasting from milliseconds to seconds are termed short-term plasticity (STP), while longer lasting changes, in the range of hours and days, are referred to as long-term plasticity (LTP) (Regehr, 2012). The capacity of neurons to adjust their synaptic strength to patterns of activity, greatly contributes to the computational power of neural circuits. Such computational power, in turn, is required for the organism to perform learning tasks and to adapt its behavioral response to changes in environmental conditions (Abbott and Regehr, 2004; Kandel, 2001)

\subsection{Basic Principles of Chemical Synaptic Transmission}

Action potential (AP)- triggered release of neurotransmitter occurs within the sub millisecond range and is dependent on the fusion of synaptic vesicles (SVs) with the presynaptic plasma membrane (Sudhof, 2013). Fusion of SVs typically occurs at the active zone, a circumscribed region of the presynaptic membrane where electron-dense material accumulates and opposes the postsynaptic density of the receiving cell that in turn harbors postsynaptic receptor complexes (Ackermann et al., 2015; Sudhof, 2012b). Active zones contain a number of highly conserved protein complexes required for the attachment of SVs to the plasma membrane (Gundelfinger and Fejtova, 2012). In addition, these areas are highly enriched in voltage dependent $\mathrm{Ca}^{2+}$ channels (VDCCs) that open upon arrival of APs at the presynaptic terminal. The opening of such leads to a steep rise in the intracellular $\mathrm{Ca}^{2+}$ concentration 
$\left(\left[\mathrm{Ca}^{2+}\right]\right)$ that ultimately accelerates the fusion rate of SVs by several orders of magnitude (Jahn and Fasshauer, 2012; Neher and Sakaba, 2008). Upon release, neurotransmitter molecules diffuse through the synaptic cleft that separates the sending from the receiving cell and elicit changes in the membrane potential of the postsynaptic cell by binding to receptor complexes at its surface.

\subsection{The Synaptic Vesicle Cycle}

A number of cell biological reactions are required to sustain the high fidelity of neuronal secretion. Before fusion, SVs are transported to the plasma membrane and undergo a process called priming to reach fusion-competence (see section 1.2.2). After fusion, SV membrane is retrieved via endocytosis and SVs are recycled to sustain future demands of transmitter release (Heuser and Reese, 1973; Rizzoli, 2014). The recycling process occurs locally within the presynaptic compartment, thereby circumventing the need for slowly operating axonal transport of de novo synthesized SVs from the soma to the nerve terminal (Kononenko and Haucke, 2015). In addition to its role in replenishing a pool of transmitter loaded SVs, endocytosis of SV membrane serves essential functions in clearing release sites for new rounds of SV fusion and in compensating increases of membrane surface after exocytosis that would otherwise lead to a loosening of membrane tension and distortions of alignment between pre- and postsynaptic specializations (Haucke et al., 2011; Hua et al., 2013; Kononenko and Haucke, 2015; Neher, 2010). Research within the last 40 years has revealed a number of different modes for endocytosis operating at different speeds within the presynaptic terminal, which likely reflects the diversity of functional requirements to sustain neurotransmission (Soykan et al., 2016). These include clathrinmediated endocytosis at low stimulation frequencies and room temperature, activity-dependent bulk endocytosis after strong presynaptic stimulation and a recently discovered ultrafast mode that only occurs at physiological body temperature and is not identical to kiss-and-run endocytosis that in turn operates in neuroendocrine cells, but seemingly not in central synapses (Delvendahl et al., 2016; Soykan et al., 2017; Soykan et al., 2016; Watanabe et al., 2013a; Watanabe et al., 2013b; Watanabe et al., 2014).

Apart from membrane retrieval, the endocytic machinery is also involved in the sorting of SV proteins (Kononenko and Haucke, 2015). This in turn is required to support the high fidelity of SVs composition and identity. However, despite the knowledge that endocytic proteins are involved in sorting SV proteins, the exact molecular pathways by which SVs maintain their molecular composition across several rounds of fusion and recycling are largely unknown. Interestingly, some evidence suggest that endosomes may be involved in supporting SV protein sorting and may thus contribute to the recycling process (Jahne et al., 2015; Rizzoli, 2014). 
Furthermore, SVs have to be reloaded with transmitter molecules by vesicular transporters in order to sustain new rounds of secretion (Bellocchio et al., 2000; Blakely and Edwards, 2012; Farsi et al., 2016; Takamori et al., 2000).

Since priming, fusion and recycling of SVs are followed by each other sequentially, all processes combined form a closed loop commonly referred to as the "synaptic vesicle cycle" (Sudhof, 2004).

\subsubsection{Synaptic Vesicle Fusion is Catalyzed by Members of the SNARE Protein Family}

SV fusion requires the action of a number of highly conserved protein families. The high energy demand of membrane merging during SV fusion (several tenths of $k_{\mathrm{B}} \mathrm{T}$ ) is fueled by the assembly of SNARE (soluble $\underline{N}$-ethylmaleimide-sensitive factor attachment protein receptor) proteins, localized on the two opposing cell compartments, into so-called trans-SNARE complexes (Gao et al., 2012; Hanson et al., 1997a; Hanson et al., 1997b; Li et al., 2007; Oelkers et al., 2016). SNAREs are characterized by a conserved stretch referred to as the SNARE motif consisting of 60-70 amino acids that is unstructured when the proteins are monomeric (reviewed in (Jahn and Scheller, 2006)). Within the SNARE complex, however, four SNARE motifs assemble in parallel fashion into a highly structured coiled coil that consists of a bundle of four alpha helices and proceeds from the $\mathrm{N}$ - to the C-terminal direction (Hanson et al., 1997b; Lin and Scheller, 1997; Sorensen et al., 2006; Sutton et al., 1998). The general architecture of the SNARE complex is highly conserved throughout evolution and members of the SNARE protein family can be classified according to their contribution to the central part of the bundle, which is composed of a stack of layers with largely hydrophobic interaction partners and a single layer of three glutamines (Qa, Qb, Qc) and one alanine (R) (Fasshauer et al., 1998; Jahn and Scheller, 2006; Sutton et al., 1998).

In the presynaptic terminal, SV fusion is carried out by the SV residing SNARE synaptobrevin and the plasma membrane residing SNAREs syntaxin-1 and SNAP-25 (Hanson et al., 1997a; Sollner et al., 1993). Synaptobrevin and syntaxin-1 are classified as R and Qa SNAREs, respectively, while SNAP-25 is a Qbc SNARE that contributes two SNARE motifs to complex formation (Fasshauer et al., 1998). The two SNARE motifs in SNAP-25 are connected to each other via a palmitoylated linker that anchors the protein to the plasma membrane. In case of synaptobrevin and syntaxin-1, C-terminal transmembrane regions mediate direct membrane localization. In addition, syntaxin-1 contains a regulatory region at its $\mathrm{N}$ terminus comprising the $\mathrm{N}$-peptide and $\mathrm{H}_{\mathrm{abc}}$ domain that is known to fold back and to bind the SNARE motif to induce a closed conformational state that inhibits SNARE complex assembly (Dulubova et al., 1999; Sudhof and Rothman, 2009). Several studies have shown that regulation of this conformational 
state plays a crucial role in gating SNARE complex assembly for SV fusion (see section 1.2.2; reviewed in (Rizo and $\mathrm{Xu}, 2015)$ ).

After fusion, cis-SNARE complexes residing at the plasma membrane need to be disassembled by the AAA+ ATPase NSF and its cofactors $\alpha$ SNAP and BSNAP in order to regenerate free SNAREs (Burgalossi et al., 2010; Sollner et al., 1993; Zhao et al., 2015).

\subsubsection{SNARE-interacting Proteins of the Munc18 Family}

A number of accessory proteins support the function of SNAREs during fusion (Sudhof and Rothman, 2009). Among these are Munc18s that belong to the family of Sec1/Munc18-like proteins (Rizo and Sudhof, 2012). Mammals express three different isoforms, of which Munc18-1 was found to be involved in neuronal exocytosis (Han et al., 2010). Deletion of Munc18-1 in mice leads to a complete shutdown in synaptic transmission thereby indicating an essential role of this protein in sustaining secretion (Verhage et al., 2000). Munc18-1 directly interacts with syntaxin-1 by binding to its $\mathrm{N}$-peptide and to its closed conformation (Dulubova et al., 2007; Dulubova et al., 1999; Misura et al., 2000; Rickman et al., 2007). The binding of the closed confirmation is thought to stabilize the inhibitory state and thus prevents SNARE complex assembly (Chen et al., 2008). In addition, functional studies have shown that this interaction is required for the chaperoning of both proteins (Zhou et al., 2013). On the other hand, the interaction between Munc18-1 and the N-peptide was reported to play a crucial role for the fusion process as such (Zhou et al., 2013). Even though the exact molecular mechanism by which this interaction contributes to the fusion reaction is still poorly understood, some evidence suggests that it contributes to the association of Munc18 to the SNARE complex throughout the fusion steps that lead to transmitter release (Rizo and Xu, 2015). The transition of the closed Munc18-syntaxin complex to an open conformation that favors SNARE complex assembly is thought to act as a gate for fusion and to be tightly regulated by the action of priming factors (Ma et al., 2011; Ma et al., 2013; Wang et al., 2017; Yang et al., 2015).

\subsubsection{Synaptic Vesicle Priming}

In order to release their contents into the synaptic cleft, SVs have to reach a fusion-competent state that allows $\mathrm{Ca}^{2+}$ triggering in the sub millisecond range. The exact nature of this state is not entirely known but some evidence suggests that it may involve the partial assembly of SNARE complexes and that it morphologically manifests itself through SV docking (Imig et al., 2014; Walter et al., 2010). Proteins of the Munc13- and CAPSs families have been described in the past to act as SV priming- and 
docking factors, respectively (Imig et al., 2014; Jockusch et al., 2007; Siksou et al., 2009; Varoqueaux et al., 2002). Both families contain a SNARE interacting MUN domain, which in case of Munc13-1 was shown to structurally be similar to protein regions found in a group of multi subunit tethering complexes (MTCs) called CATCHRs (Complex Associated with Tethering Containing ㅂelical Rods) (Li et al., 2011; Pei et al., 2009; Yang et al., 2015). These in turn have been described to bridge the distance between cellular compartments by interacting with SNAREs, regulatory lipids and vesicular components such as Rab GTPases and coat proteins (Yu and Hughson, 2010). Thus, mechanisms by which CAPSs and Munc13s prime SVs may reflect adaptations of an ancient tethering process that has diversified in evolution to meet the needs of various trafficking steps in cells (Pei et al., 2009). In the following sections, I will give an overview on the domain structure and the known functions of these two protein families.

\subsubsection{Proteins of the Munc-13 Family}

Munc13s are large proteins ( $120-200$ kDA) that constitute a family with five known isoforms (Augustin et al., 1999; Betz et al., 1998; Betz et al., 2001; Brose et al., 1995; Koch et al., 2000; Shiratsuchi et al., 1998). All of these isoforms (Munc13-1, ubMunc13-2, bMun13-2, Munc13-3, Munc13-4, BAIAP3) contain a $\mathrm{Ca}^{2+}$-dependent phosphatidylinositol-4,5bisphosphate $\left(\mathrm{PIP}_{2}\right)$ binding $\mathrm{C2}$ domain $(\mathrm{C} 2 \mathrm{~B})$, a central SNARE-binding MUN domain and a C-terminal C2C domain that does not bind $\mathrm{Ca}^{2+}$ (Koch et al., 2000; Pei et al., 2009; Shin et al., 2010). The N-terminal region of Munc13s is variable and in case of Munc13-1, ubMunc13-2, bMunc13-2 and Munc13-3 contains a diacylglycerol (DAG) binding C1 domain and a calmodulin binding region that are absent from Munc13-4 and BAIAP3 (Fig.2) (Betz et al., 1998; Junge et al., 2004; Lipstein et al., 2012; Rodriguez-Castaneda et al., 2010; Shen et al., 2005; Sudhof, 2012b). In addition, Munc13-1 and ubMunc13-2 harbor an N-terminal C2A domain that is known to bind active zone proteins of the RIM family (ㅁab3 interacting molecules) (Betz et al., 2001; Deng et al., 2011; Dulubova et al., 2005).

The three most prominently expressed isoforms in the brain are Munc13-1, ubMunc13-2 and bMunc13-2. Genetic deletion of these leads to a complete abrogation of transmitter release in cultured hippocampal neurons (Varoqueaux et al., 2002). Similarly, synaptic transmission becomes largely perturbed upon deletion of the c.elegans homologue unc13 leading to a severe, uncoordinated motor phenotype in worms (Brenner, 1974; Richmond et al., 1999). 


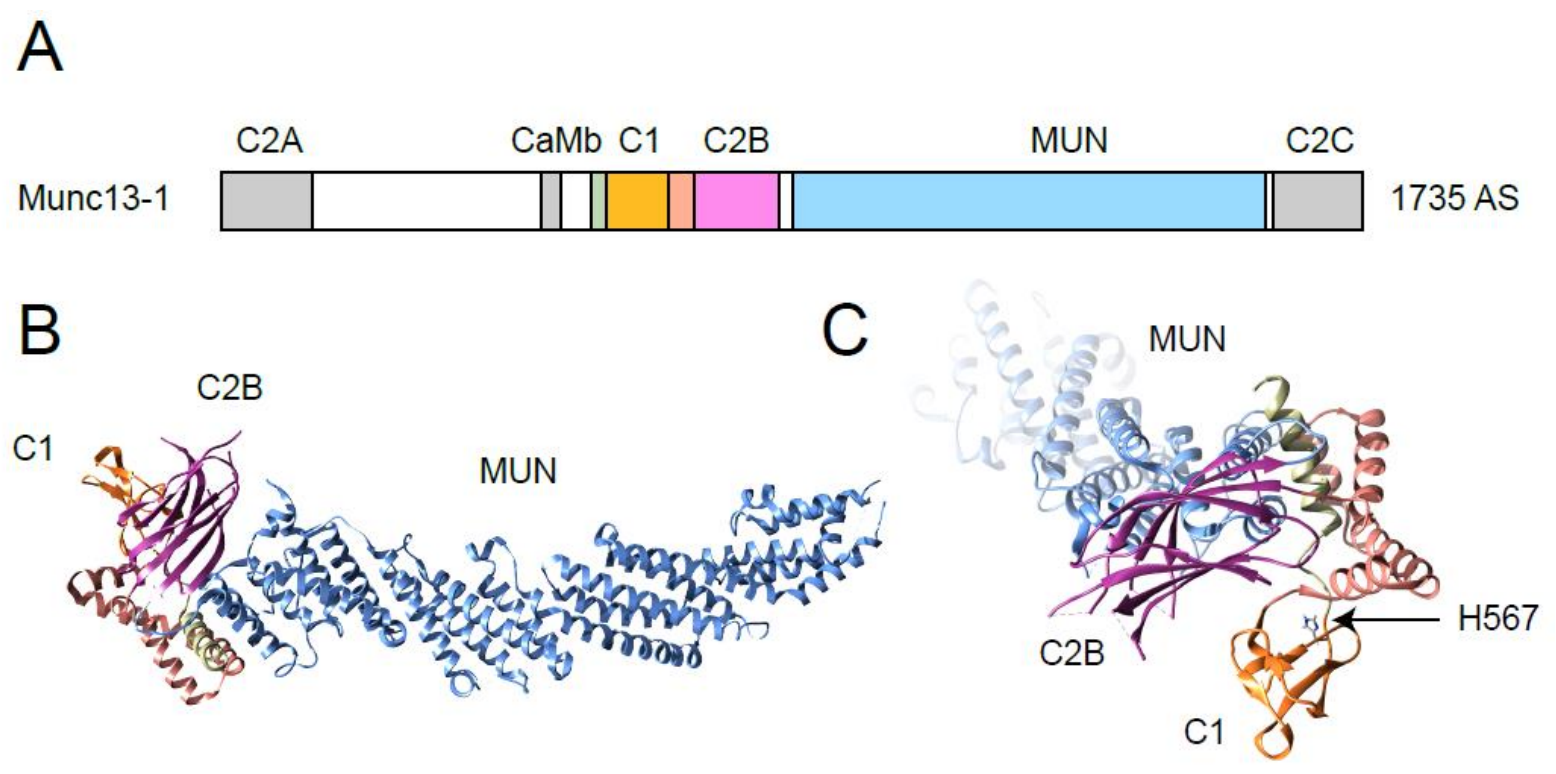

Figure 1: Domain structure of Munc13-1. A) Overview of the Munc13-1 domain organization (adapted from Xu et al., 2017). B) Three dimensional crystal structure of the Munc13-1 C1,C2B, MUN protein region. (PBd 5ue8; structure analysis was performed using MegAlign Pro, DNASTAR Incorp.) (C) Detailed illustration of the C1 and C2B domain structure. The arrow indicates the position of the histidine residue that was exchanged with lysine in several studies to disrupt the interaction between DAG and the C1 domain of Munc13-1 (Basu et al., 2007; Betz et al., 1998; Hu et al., 2015; Rhee et al., 2002)

The first mechanistic insights into the function of Munc13s came from results that demonstrated binding of Munc13s to the N-terminal part of syntaxin (Betz et al., 1997). Other results were published shortly after this discovery that suggested a potential role of Munc13s in inducing changes in the interaction between Munc18 and syntaxin (Sassa et al., 1999). These two initial findings led to the hypothesis that Munc13s may trigger the opening of the closed syntaxin conformation to initiate SNARE complex formation (Brose et al., 2000). First experimental evidence for this model came from a study on unc13 mutant worms, in which expression of a constitutively open syntaxin protein (the so-called LE mutant) restored transmitter release to wild type (WT) control levels (Richmond et al., 2001). Other studies employing biochemical methodologies such as NMR and artificial liposome fusion assays provided further support of the initial hypothesis and suggested an essential involvement of the MUN domain in this (Fig.2) (Basu et al., 2005; Ma et al., 2011; Ma et al., 2013; Yang et al., 2015). Most recent results, obtained from single molecule fluorescence energy transfer (smFRET) experiments suggest that Munc13s induce a conformational change within the linker region of syntaxin, which connects its SNARE motif the N-terminus (Wang et al., 2017). This change in turn is speculated to be required for the opening of syntaxin and to promote SNARE complex assembly (Wang et al., 2017).

Apart from their function in opening syntaxin, Munc13s may mediate other steps that are required for the fusogenecity of SVs. Interestingly, the finding that the LE mutant is sufficient to restore transmitter release in unc13 mutant worms has been challenged by the results of a more recent study (McEwen et 
al., 2006). There, the authors found that stimulus evoked responses remain largely perturbed upon overexpression of the LE-construct, in contrast to what was published previously using the same mutant strain (McEwen et al., 2006). At present it is not clear whether the discrepancy in results arose from differences in recording conditions, but the additional finding that the lethal Munc13-1,2 doubleknockout (DKO) phenotype cannot be rescued by introduction of the LE mutation into mice rather supports the notion that the opening of syntaxin as such may not be sufficient to compensate for a loss of Munc13s (Gerber et al., 2008). Furthermore, while it was initially reported that strong overexpression of a Munc13-1 fragment that only comprises the MUN domain can partially restore transmitter release in cultured Munc13 DKO neurons, observations by others using the same experimental approach contradicted these findings suggesting that the MUN domain on its own is not sufficient to sustain priming (Rhee JS, unpublished results; see also (Liu et al., 2016) for rescue experiments, in which the MUN domain was expressed at physiological levels). Therefore, it is unlikely that opening of syntaxin by the MUN domain may entirely account for the priming process and Munc13s may thus be required for additional steps.

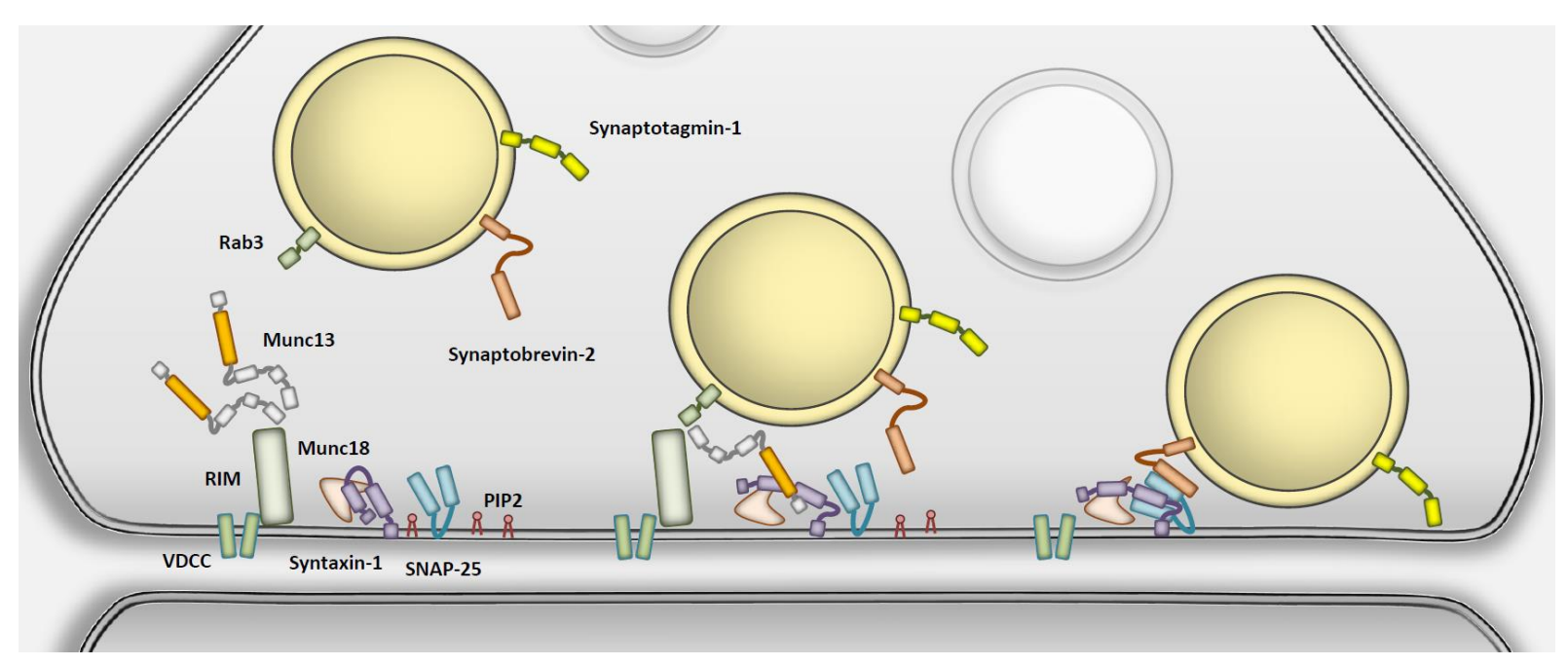

Figure 2: Hypothetical model of the Munc13 dependent priming process. Munc13-1 is recruited to the active zone by RIM proteins via its C2A domain. The MUN domain of Munc13s is thought open the closed Syntaxin/Munc18 complex and may thus facilitate SNARE complex assembly.

Even though little is known about additional functions, results from two recent studies suggest that Munc13s are further involved in bridging the distance between SVs and the plasma membrane (Liu et al., 2016). This bridging presumably occurs at low intraterminal $\left[\mathrm{Ca}^{2+}\right]$ and it is accomplished by the simultaneous binding of the $\mathrm{C} 1, \mathrm{C} 2 \mathrm{~B}$ and $\mathrm{C} 2 \mathrm{C}$ domains to plasma membrane- and SVs lipids. However, the exact interactions that mediate such bridging are still unknown and it is not clear at present whether 
$\mathrm{Ca}^{2+}$ dependent binding of $\mathrm{DAG}$ and $\mathrm{PIP}_{2}$ by the $\mathrm{C} 1$ and $\mathrm{C} 2 \mathrm{~B}$ domains may regulate the bridging or rather modulate downstream processes that are directly linked to SNARE complex assembly. Interestingly, mutations in Munc13s that interfere with the binding of DAG or $\mathrm{PIP}_{2}$ lead to stronger short-term synaptic depression in cultured neurons, indicating that these interactions fulfill regulatory functions rather than being essential for the priming process as such (Rhee et al., 2002; Shin et al., 2010).

\subsubsection{Proteins of the CAPS Family}

Proteins of the CAPS ( $\underline{\mathrm{Ca}}^{2+}$ dependent activator protein for secretion) family comprise two members that share the same domain structure (Speidel et al., 2003; Stevens and Rettig, 2009). The N-terminal half harbors a $\mathrm{C} 2$ domain, which does not bind $\mathrm{Ca}^{2+}$ at physiologically relevant levels, and a pleckstrin homology (PH) domain that interacts with PIP 2 (Ann et al., 1997; Grishanin et al., 2002; James et al., 2008; Martin, 2015). The C-terminal half contains a SNARE-interacting MUN domain and a stretch of amino acids that interacts with acidic phospholipids and targets CAPSs to DCVs (Daily et al., 2010; Grishanin et al., 2002; James and Martin, 2013; Kabachinski et al., 2016; Khodthong et al., 2011). CAPS1 and 2 are differentially expressed in the brain, with CAPS1 being the dominant of the two isoforms (Sadakata et al., 2006; Speidel et al., 2003).

CAPS1 ( 145 kDa) was first identified as a regulator of secretion in a biochemical study in which the authors screened for cytosolic factors that promote $\mathrm{Ca}^{2+}$ dependent exocytosis in permeabilized PC12 cells (Martin and Walent, 1989; Walent et al., 1992). Shortly after this seminal discovery, CAPS1 was found to constitute a homologue of Unc31, which had been described to regulate neural secretion in C.elegans (Ann et al., 1997; Avery et al., 1993; Miller et al., 1996). Together, these early findings led to the hypothesis that CAPS1 acts as a highly conserved component of the release machinery.

In the following years, CAPS1 was speculated to specifically regulate DCV exocytosis (Berwin et al., 1998; Hammarlund et al., 2008; Renden et al., 2001; Speese et al., 2007; Tandon et al., 1998). Strongest support for this came from studies, which showed that CAPS1 localizes to DCV membranes, but not to SVs, and that secretion of glutamate from semi intact synaptosomes is not perturbed upon its removal (Berwin et al., 1998; Tandon et al., 1998). In addition, neurons from mutant unc31 worms were shown to exhibit a pronounced DCV docking deficit, while SV docking was not changed in comparison to control levels (Hammarlund et al., 2008).

Despite these findings, evidence has accumulated in recent years that CAPSs regulate SV exocytosis as well. Notably, one study reported a $50 \%$ reduction in the amplitude of stimulus evoked postsynaptic currents in the neuromuscular junction of unc13 mutant worms (Gracheva et al., 2007). Moreover, synaptic transmission has been found to be almost completely abolished in cultured cells from the 
hippocampus of CAPS1,2 DKO mice, indicating that CAPSs are required to sustain fast glutamatergic transmitter release (Jockusch et al., 2007). This strong perturbation has also been shown to become manifest as a dramatic reduction in the number of docked SVs revealed by 3D electron tomography of high-pressure frozen tissue samples from mutant CAPS DKO mice (Imig et al., 2014). While these phenotypic changes closely resemble those of Munc13 DKO neurons, transmitter release can transiently be restored in CAPS DKO cells by high frequency stimulation (HFS) or by the DAG analog phorbol 12,13dibutyrate (PDBu) in contrast to Munc13 DKO neurons (Jockusch et al., 2007). Thus, strong stimuli, such as depolarizing neurons with high $\mathrm{KCl}$, which is commonly used to study SV recycling, may mask the strong synaptic phenotype of mutant CAPS/Unc31 cells and may accordingly have led to some of the results of earlier studies that did not detect an essential role of CAPS in regulating SV exocytosis (Speese et al., 2007).

The molecular mechanisms by which CAPSs regulate transmitter release are poorly understood. Similar to Munc13s, CAPSs are capable of binding SNAREs via their MUN domain (Daily et al., 2010; James et al., 2009; Khodthong et al., 2011; Parsaud et al., 2013). The finding that the DCV docking deficit in unc31 mutant neurons can be rescued by the syntaxin-LE mutant has led to the hypothesis that CAPSs may prime secretory vesicles by modulating the conformational state of syntaxin (Hammarlund et al., 2008). Interestingly, results from one study indicated that the C-terminal half of the MUN domain of CAPSs binds syntaxin in a mode that is distinct from that of Munc13s and may thus account for the nonredundant functions of these proteins in the priming process (Parsaud et al., 2013). Specifically, CAPS1 was found to bind full length syntaxin in its open conformational state and may thus stabilize it to facilitate SNARE complex assembly (Parsaud et al., 2013). Yet, other studies reported a different binding mode of CAPSs to syntaxin, which involves the N-terminal half of the MUN domain of CAPSs and the Cterminal SNARE motif and membrane linker of syntaxin (Daily et al., 2010; James and Martin, 2013; Khodthong et al., 2011). These studies also detected interactions between CAPSs and the other two SNARE proteins (synaptobrevin and SNAP25) leading to the hypothesis that CAPSs may directly orchestrate SNARE complex assembly by simultaneously binding to all SNAREs (Daily et al., 2010; James and Martin, 2013; Khodthong et al., 2011).

In the light of these differences in findings, it remains unclear how CAPSs primes secretory vesicles. Notably, other domains in CAPSs, have been described to essentially contribute to transmitter release in PC12 cells, including the PIP 2 binding PH domain, the DCV binding site and the $\mathrm{C} 2$ domain, which has been described to regulate the dimerization of CAPSs (Grishanin et al., 2002; James et al., 2008; Petrie et al., 2016). At present it is not clear, whether these domains are solely required to sustain the MUN domain in the priming process, or whether these are distinct functional modules that mediate other steps that lead to transmitter release. 


\subsubsection{SYD1A and Mover}

Apart from CAPSs and Munc13s, other proteins have been described to regulate release probability and SV docking. Among these are two recently identified presynaptic proteins, namely mSYD1A and Mover (Ahmed et al., 2013; Korber et al., 2015; Kremer et al., 2007; Wentzel et al., 2013). mSYD1A was shown to interact with Munc18 and to resemble some distant homology to the C.elegans SYD1 (Wentzel et al., 2013). Interestingly, deletion of mSYD1A leads to a 50\% reduction in the number of docked SVs in neurons from acute hippocampal slices (Wentzel et al., 2013). At present, it is not known, whether this reduction is also accompanied by a decrease in stimulus evoked EPSCs and whether mSYD1A functionally cooperates with Munc13s and CAPSs during the priming process. Mover, in contrast, has not been described to regulate SV docking. However, it was shown to bind calmodulin, similar to Munc13s, and to regulate STP by modulating the $\mathrm{Ca}^{2+}$ sensitivity of release (Korber et al., 2015). Taken together, both of these proteins may functionally cooperate with Munc13s and CAPSs during the SV priming process.

\subsection{Relevance of SV priming for synaptic plasticity}

Functionally, primed vesicles constitute the so-called readily-releasable pool (RRP) whose size can be determined electrophysiologically by applying short and strong pulses that deplete all readily available SVs and measuring the resultant increase in membrane capacitance presynaptically, or the current that is evoked postsynaptically (Neher, 2015; Rosenmund and Stevens, 1996; Scheuss et al., 2002; Thanawala and Regehr, 2013). Even though many studies have treated the RRP as consisting of a homogeneous population of SVs with identical release probabilities, emerging evidence suggests that there are subpopulations of RRP vesicles that differ from one another with regards to their spatial localization to clusters of VDCCs, their degree of fusogenicity and their priming rate (reviewed in (Neher, 2015, 2017)). Some of this heterogeneity has been found to be directly linked to the action of Munc13s and to be of functional significance for STP (Lee et al., 2013; Lipstein et al., 2012; Taschenberger et al., 2016).

STP, in turn, is thought to be involved in many brain functions, such as sensory adaptation (Abbott and Regehr, 2004; Chung et al., 2002; Lampl and Katz, 2017). The importance of STP for normal brain function is particularly demonstrated by the finding that altered forms of STP are contributing to the etiology of psychiatric diseases (Crabtree and Gogos, 2014). Interestingly, a recent study identified a mutation in the human homologue of Munc13-1 in an autistic patient, which leads to more pronounced short-term depression (STD) in cultured neurons when compared to WT-Munc13 expressing cells (Lipstein et al., 2017). This finding indicates that that the synaptic vesicle priming process is important to fine tune synaptic transmission for normal brain function. Other studies have also identified 
mutations in the human homologue of CAPS1, yet it has not been addressed, whether any of these lead to altered STP as well (Sitbon et al., unpublished results) (Sadakata et al., 2013; Sadakata et al., 2007b).

\subsection{Aim of the study}

In the present study, I aimed at contributing to a better understanding of the SV priming process. For this, I studied the function of mSYD1A and Mover in synaptic transmission in cultured neurons from mutant KO mice. From these experiments, I anticipated to gain insights as to whether these proteins are involved in sustaining the CAPS and Munc13 dependent priming process.

Furthermore, I aimed at elucidating the functional interplay between CAPSs and Munc13s. At present, it is not known how these two factors functionally cooperate to render SVs fusion competent. Notably, previous studies indicated that CAPSs and Munc13s may fulfill non redundant functions. Thus, one aim of this study was to shed more light on the distinct contribution of each of these proteins to the priming process. For this, I particularly focused my attention on a detailed structure function study of CAPS in the context of glutamatergic synaptic transmission, which had not been carried out before. Included in this study were a number of mutations that were found in BPD patients. Accordingly, I not only expected to gain greater insights into the priming process, but further I anticipated to contribute to a better understanding of the etiology of the disease.

In the last part of this study, I aimed at establishing an experimental approach that would allow the future study of STP in the context of visual information processing. As described above, STP is thought to be crucially involved in normal brain function. I thus expected that this particular part of my study could help in the future to gain greater insights into the contribution of STP so sensory processing. 


\section{Methods}

\subsection{Materials}

Table1: Chemicals for cell culture and electrophysiology

\begin{tabular}{|c|c|}
\hline Chemical & Company \\
\hline Acetic acid & Merck \\
\hline Adenosine 5'-triphosphate (ATP) magnesium salt & Sigma Aldrich \\
\hline Agarose type Ila, medium & Sigma Aldrich \\
\hline Albumin, bovine & Sigma Aldrich \\
\hline Biocytin & Sigma Aldrich \\
\hline B27 supplement & Gibco \\
\hline Calcium chloride $\left(\mathrm{CaCl}_{2}\right)$ & Merck \\
\hline Cesiummethanesulfonate & Sigma Aldrich \\
\hline Collagen 1 , rat tail & Becton Dickinson GmbH \\
\hline Creatine phosphokinase from rabbit muscle & Sigma Aldrich \\
\hline L-Cysteine & Sigma-Aldrich \\
\hline Dulbecco's Modified Eagle Medium (DMEM) & Gibco \\
\hline DMEM with GlutaMAx & Gibco \\
\hline Ethylenediaminetetraacetate (EDTA) & Merck \\
\hline Fetal bovine serum (FBS) & PAA Laboratories GmbH \\
\hline GlutaMAx & Gibco \\
\hline Guanosine triphosphate (GTP) sodium salt & Sigma Aldrich \\
\hline HEPES & Sigma Aldrich \\
\hline Hydroxyethyl piperazineethanesulfate (HEPES) & Sigma Aldrich \\
\hline Ethylene glycol tetraacetic acid (EGTA) & Merck \\
\hline Magnesium chloride $\left(\mathrm{MgCl}_{2}\right)$ & Merck \\
\hline MITO + serum extender & Becton Dickinson GmbH \\
\hline Neurobasal A & Gibco \\
\hline Papain & Worthington Biomedical Corp. \\
\hline Penicillin / streptomycin & Gibco \\
\hline Phosphocreatine disodium salt hydrate & Sigma Aldrich \\
\hline
\end{tabular}




\begin{tabular}{ll}
\hline Poly-D-lysine & Sigma Aldrich \\
\hline Potassium D-gluconate & Sigma Aldrich \\
\hline Sodium chloride $\left(\mathrm{NaCl}_{2}\right)$ & Merck \\
\hline Sucrose & Merck \\
\hline Tedrodotoxin (TTX) & Tocris Bioscience \\
\hline Tetraethylammonium chloride (TEA-Cl) & Sigma Aldrich \\
\hline Trypsin-EDTA (0.05\% (wt/vol) & Gibco \\
\hline Trypsin inhibitor & Sigma Aldrich \\
\hline
\end{tabular}

\subsection{Generation of CAPS1 ${ }^{\mathrm{dEX} 2}$ mice}

The genetic deletion of exon 2 of CAPS1 and of putative splice acceptor sites ( $\sim 30$ bp of intronic sequence flanking each site of the exon) was carried out by using the novel CRISPR methodology (Cong et al., 2013; Wang et al., 2013). The CRISPR/CAS9 system was initially discovered in prokaryotes, where it mediates adaptive immune responses against viral pathogens (Brouns et al., 2008; Marraffini, 2015). In detail, small parts of the viral genome are integrated into the host genome as so-called spacers that are transcribed into small pre-CRISPR RNAs (crRNAs). These additionally contain short palindromic repeats that are complementary in their sequence to another type of RNA called trans-activating crRNA (tracrRNA). Both of these RNAs form a complex that is processed into short RNA guides that direct DNAses of the CAS9 type to viral DNA for strand breaking. In order to employ this system for genome editing in mammals, human-codon optimized CAS9 (hCAS9) mRNA is injected into cells together with a chimeric fusion construct of crRNA and tracrRNA called single-guide RNA (sgRNAs) (Cong et al., 2013; Ran et al., 2013). Upon double-strand break, DNA can undergo two different repair mechanisms. One mechanism, called non-homologues end joining (NHEJ) is prone to errors and leads often to shifts in the reading frame, while another, called homology-directed repair (HDR), yields high fidelity repair of the DNA sequence (Ran et al., 2013). In order to facilitate HDR and to introduce site specific modifications such as the exchange of bases, DNA templates can be injected into cells together with the CRIPSR components.

For the genetic deletion of exon 2 in CAPS1, I chose a guide-RNA sequence with the help of an online tool provided by the Zhang lab (Massachusetts Institute of Technolgy; crispr.mit.edu). According to the web tool, this sequence had a quality score of 90 indicating a low likelihood of off-target binding.

The following experimental procedures were carried out by Fritz Benseler and his team. 


\subsubsection{Generation of sgRNA}

SgRNA was prepared by extension PCR and T7 in-vitro transcription using the following primers:

\section{Forward}

5'-TTAATACGACTCACTATAGGACTGTCAAAGACCGGTTCCGTTTTAGAGCTAGAAATAGC-3'

\section{Reverse}

5'-AAAAGCACCGACTCGGTGCCACTTTTTCAAGTTGATAACGGACTAGCCTTATTTTAACTTGCTATTTCTAGCTC TAAAAC-3'

The green script in the forward primer highlights the T7 promotor sequence and the blue script indicates the sequence encoding for the guide RNA. The in-vitro transcription of DNA into RNA was carried out based a protocol provided by the manufacturer (Cellscript).

\subsubsection{Generation of hCAS9 mRNA}

In order to obtain hCAS9 mRNA, the following primers were used to amplify DNA sequences from a plasmid encoding hCAS9 (Addgene_42230_pX330-U6-Chimeric_BB-CBh-hSpCas9).

\section{Forward}

5'-TAATACGACTCACTATAGGGAGAATGGACTATAAGGACCACGAC-3'

\section{Reverse}

5'-GATGGCTGGCAACTAGAAGG-3'

The amplified PCR product was subjected to T7 in-vitro RNA transcription and poly-A tailing, as well as $5^{\prime}$ capping were applied using commercially available protocols to obtain mature mRNA.

\subsubsection{Generation of DNA template for HDR}

In order to specifically induce the deletion of exon 2 and putative splice acceptor sites, PCR amplification of genomic DNA in combination with Gibson assembly (NEB) was used to generate a DNA template for HDR spanning the $5^{\prime}$ and $3^{\prime}$ flanking regions next to the putative splice acceptor sites: 
5'-AATCTGAAAAGAGACATGACCCACTTATCTACAGATTCTATCTTACAAGCACTGTGCCTATAAATAACTACAG GCACAAATTAAAGTAAGAAATGAAAAAAATAGCAATTTCTGAATATAACCAAGCATCACTCCCTAAGAAATGTTT TAACAGCCAACTCTTTCAAAAGTTAGAAATAAACAAACAAGCAAACAAACAAACCTACTTGACAGCACTTGCTGA TTTCTGTGGTAACAGAATTCTTGTGTGGTTGTAAAGCAATGTATTAAACTGTGGCTTGTACTATTTTTAGCAATGA ATGTGTTCCATGTGAGTTCAGAAAGTTTGCTCATTTATCAGGCCAGCCCACACTCTGATAGATGGGCATCTTTCT TGTGAAGTAGTCACATCCCTGTTTACATGAA***CCTAGCCTACTGATCTGATAAAGACTGCAGAGGGTGTAGCA CCTTGGCTGATGCAGAGGTGTCAGTAGTCATCTGACAAGGGACTCTATAGTTGTGTTGTAGAGAATGATGCAAA TAGCTCCTGCAGTTGGGCATTGGACTGATGACTCGGTATGCAGAGTGCTTGACACATAAGCCTGAAGACTTGAG TGGAATCCTTAGTTCCACATACAAGTCTGAGTTCAGTGTTGAATGCTTGCAATCCCAGTGCTGGGGAGATGGAG ACAGGAGGGTTCCTGAGGTTCATTGGTGTCTTAGTTAGTGTTCTGTTGCTATGAAGAGACACCATGACCATGGC AACTCTTATAGAGAAAAAGCATTTGATTGTGGCTTACTCAC-3'

*** $=$ deletion-side (exon 2 \& putative splice acceptor sites).

\subsubsection{Injection}

The injections of the CRISPR components into pronuclei of zygotes were carried out by Ursula Fünfschilling. The injection mix consisted of $10 \mathrm{ng} / \mu \mathrm{L}$ hCAS9 mRNA, $5 \mathrm{ng} / \mu \mathrm{L} \mathrm{sgRNA}$ and $20 \mathrm{ng} / \mu \mathrm{L}$ template DNA diluted in $5 \mathrm{mM}$ Tris $\mathrm{HCl}(\mathrm{pH} 7.4)$.

\subsubsection{Mouse Genotyping}

The following primers were used for the genotyping of mice:

\section{Forward ("primer sense")}

5'_AATCTGAAAAGAGACATGACCC_3'

\section{Reverse Primer ("primer antisense")}

5'_GTGAGTAAGCCACAATCAAATGC_3'

The genetic deletion typically yielded DNA bands at $~ 800 \mathrm{bp}$ and a band at $1 \mathrm{~kb}$ was indicative of a wild type allele. 


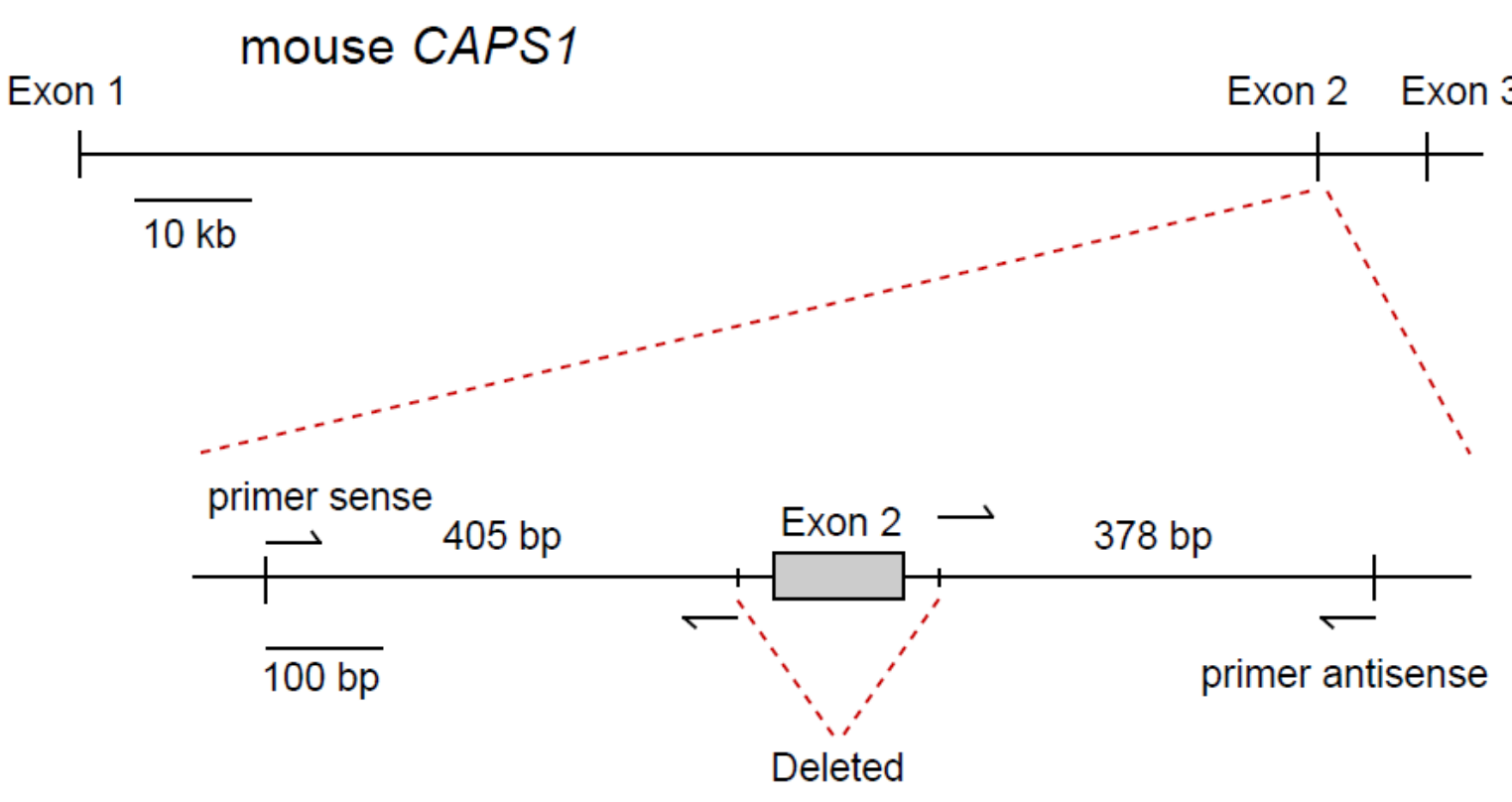

Figure 3: Summary of genetic manipulation. Deletion of exon 2 and putative splice acceptor sites in CAPS1 was performed using the CRISPR/CAS9 system. Primers sense and antisense were used for genotyping.

\subsection{Generation of cCAP1 KO mice}

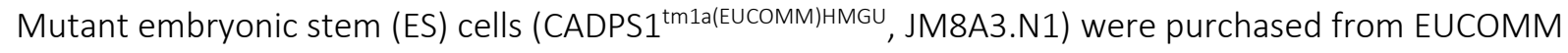
(European Conditional Mouse Mutagenesis Program) in order to generate a conditional CAPS1 KO (cKO) mouse line. These cells contained an L1L2_Bact_P cassette (FRT-EN2,IRES,lacZ,pA-loxPhbactP, neomycin,pA-FRT-loxP) in chromosome 14 upstream of exon 10 of CADPS1 at position 12529023 and an additional loxP site downstream of exon 10 at position 12528182. The preparation of ES cells for blastocyst injections was carried based on a protocol provided by EUCOMM. Chimeric mice were crossed with a mouse strain expressing Flp recombinase for the removal of the neomycin cassette, thereby allowing the conditional excision of exon 10 by cre recombinase.

\subsection{Cell culture and site directed mutagenesis}

Autaptic hippocampal cultures were prepared according to a published protocol (Burgalossi et al., 2012). Neurons were either isolated from E18 embryos or from PO mice and were used for experiments between DIV-10 and DIV-14. The mutagenesis of CAPS2 CDNA was performed using the Quikchange Lightning Site-Directed Mutagenesis kit from Agilant. 


\subsection{Virus Production}

Lentiviruses were prepared as described in the literature (Ripamonti et al., 2017). In brief, HEK293FT cells were grown on $15 \mathrm{~cm}$ Poly-L-Lysine coated dishes at a confluence of 90\%. A transfection mixture consisting of $40 \mu \mathrm{g}$ template-, $16 \mu \mathrm{g}$ pVSVG- and $16 \mu \mathrm{g}$ pCMV R8.9 DNA was prepared in $6 \mathrm{~mL}$ OptiMem medium and incubated for $5 \mathrm{~min}$ at room temperature. Another mixture consisting of $60 \mu \mathrm{L}$ Lipofectamin 2000 (Invitrogen) and $6 \mathrm{~mL}$ OptiMem was added to this and the reaction mixture was incubated for $1 \mathrm{~h}$ at room temperature. The $12 \mathrm{~mL}$ transfection reaction was then transferred dropwise into the cell culture dish and the media was replaced by DMEM (+2\% FCS/ 1\% PenStrep/ $10 \mathrm{mM}$ NaButyrate) after a 6 hour incubation at $37^{\circ}$. The virus was harvested after $48 \mathrm{~h}$ from cell culture medium and concentrated to a volume of $500 \mu \mathrm{L}$ in TBS by centrifugation using $100 \mathrm{kDa}$ Amicon Ultra15 Centrifugal Filter units (Millipore). Aliquots of $50 \mu \mathrm{L}$ were flash frozen in liquid nitrogen and stored at $-80^{\circ} \mathrm{C}$.

Semliki Forest Viruses were prepared according to a published protocol (Ashery et al., 1999). The invitro transcription of cDNA to generate RNA for electroporation into cultured BHK cells was carried out using the mMessage mMachine SP6 kit from Ambion.

\subsection{In-vitro Physiology}

Whole-cell recordings in cultured autaptic hippocampal neurons were carried out as described before (Burgalossi et al., 2012; Nair et al., 2013). In brief, cells were voltage clamped at -70 mV using a Multiclamp 700 B amplifier under the control of the pClamp software (Molecular Devices). The data was sampled at a rate of $10-25 \mathrm{kHz}$ and the series resistance was compensated by $20-70 \%$. Only cells with series resistances $\leq 12 \mathrm{M} \Omega$ were included in the analysis. In order to measure AP-triggered EPSCs, the membrane potential was depolarized for $2 \mathrm{~ms}$ from $-70 \mathrm{mV}$ to $0 \mathrm{mV}$. Cultured neurons were constantly supplied with fresh extracellular bathing solution via a perfusion system. Pharmacological agents were applied using a fast flow application system consisting of valve controlled capillaries and a stepper device (SF-77, Warner Instrument). Spontaneously occurring mEPSCs were measured in presence of 300 $\mathrm{nM}$ tetrodotoxin and the RRP size of neurons was determined by applying $500 \mathrm{mM}$ sucrose solution for 6-8 s as published previously (Basu et al., 2007; Jockusch et al., 2007; Rosenmund and Stevens, 1996). The following solutions were used for experiments:

\section{$\underline{\text { Intracellular solution }}$}

$136 \mathrm{mM} \mathrm{KCl}, 17.8 \mathrm{mM}$ HEPES, $15 \mathrm{mM}$ Phosphocreatine, $1 \mathrm{mM}$ EGTA, $0.6 \mathrm{mM} \mathrm{MgCl}$, $0.3 \mathrm{mM}$ Na-GTP, 4 mM Mg-ATP, $5 \mathrm{U} / \mathrm{mL}$ Creatinephosphokinase (solution adjusted to $\mathrm{pH}$ 7.4; $320 \mathrm{mOsmol} / \mathrm{L}$ ). 
Extracellular solution ("Base+")

$140 \mathrm{mM} \mathrm{NaCl}, 4 \mathrm{mM} \mathrm{CaCl}$, .4 mM KCl, 10 mM HEPES, 24 mM MgCl, 10 mM Glucose (solution adjusted to $\mathrm{pH} 7.3, \sim 310 \mathrm{msOsmol} / \mathrm{L})$

\subsection{In-vivo Physiology}

In order to carry out whole cell recordings from the visual cortex of anaesthetized mice, I built an electrophysiological recording setup that consisted of the following components (Tab.2):

Table 2: Equipment for in-vivo patch clamp recordings

\begin{tabular}{lll}
\hline Component & Application & Company \\
\hline Multiclamp 700 B & Amplifier & Molecular Devices \\
\hline Digidata 1440 & Digitizer & Molecular Devices \\
\hline CV-7B & Headstage & Molecular Devices \\
\hline Unit MRE/MLE & Micromanipulator & Luigs and Neumann \\
\hline Remote Control SM7 & Micromanipulator & Luigs and Neumann \\
\hline Control Box SM7 & Micromanipulator & Luigs and Neumann \\
\hline Round Pillar ø40 mm, magnet & Fixation of & Luigs and Neumann \\
(micromanipulator) & Micromanipulator unto & \\
& table & \\
\hline Vision Isostation & Vibration table & Newport \\
\hline Manuel-Seal-Sucker & Air pressure sensoring & Sigmann Elektronik \\
\hline NIM8306 & (NIM crate for power & CAEN S.p.A. \\
& supply of Seal-Sucker & \\
\hline Stemi 2000 & Binocular & Zeiss \\
\hline Basic Stand 250 mm, inclinable & Flexible arm for binocular & Pulch \& Lorenz \\
\hline KL 300 LED & Lightsource & Schott \\
\hline LED flexible lightguide 1-branch & Light guide & Schott \\
\hline DG Holder System & Fixation of light guide & NOGA / Sartorius \\
& & Werkzeuge GmBH \\
\hline EZB 800 Vaporizer Kit & Anaesthesia control & WPI \\
\hline DC Temperature Controller & Body temperature control & FHC \\
\hline Mini Rectal Thermistor Probe & Body temperature control & FHC \\
\hline 6.5x9.5 cm Heating Pad & Body temperature control & FHC \\
\hline Omnidrill35 & Driller for Craniotomy & WPI \\
\hline Mouse/Neonatal Rat Adaptor & Stereotactic fixation & WPI \\
\hline Optical Mono fiber & Light stimulation & Doric Lenses \\
\hline Connectorized Single LED (LEDC1-B_FC) & Light stimulation & Doric Lenses \\
\hline T-Cube LED Driver (LEDD1B) & Light stimulation & Thorlabs \\
\hline IC light to voltage sensor, 3 PIN (TSL253-R- & Photodiode & Digi Key \\
LF-ND) & & \\
\hline & & \\
\hline
\end{tabular}




\subsubsection{Mouse surgery and in-vivo blind patch clamp recordings}

All procedures were carried out in accordance with the Max-Planck society guidelines and with approval of the federal state of lower Saxony (Niedersächsisches Landesamt für Verbraucherschutz und Lebensmittelsicherheit).

The surgery of mice and blind patch clamp recordings were carried out with modifications according to published methodologies (Crochet, 2012; Margrie et al., 2002; Narayanan et al., 2014). Mice were anaesthetized using isoflurane and positioned unto a heating pad for stereotactic fixation. The depth of anesthesia was monitored throughout surgical- and experimental procedures by assessing reflexes in response to toe pinching and the breathing rate of mice was adjusted to $\sim 100$ breaths per seconds by controlling the concentration of isoflurane via a vaporizer system. The body temperature of mice was held constant at $37^{\circ} \mathrm{C}$ and was monitored with a rectal probe and a heat sensor. Metamizol was applied as analgesic at a concentration of $200 \mathrm{mg} / \mathrm{kg}$ bodyweight and an incision across the midline was made to expose the skull. After cleaning of the skull, a recording chamber was forged using kwik-cast sealing (WPI). In order to perform recordings in the monocular region of the visual cortex of mice, a $0.5 \mathrm{~mm} x$ $0.5 \mathrm{~mm}$ craniotomy was introduced $3 \mathrm{~mm}$ posterior of bregma and $2.5 \mathrm{~mm}$ lateral to the midline by thinning the skull with a dental drill and removing the bone and parts of the dura mater with a surgical needle. The recording chamber was then filled with fresh Locke's solution and patch pipettes (4-6 M $\Omega$ ) were inserted into the brain tissue with a pressure of $\sim 180-200$ mbar under the visual control of a binocular. A test pulse was applied in the voltage clamp mode and changes in the amplitude of the currents recorded by the patch pipette were constantly monitored. The penetration of the pia typically resulted in a transient decrease in the current pulse and was used a reference for the recording depth. Once a depth of $350 \mu \mathrm{m}$ was reached, the pressure was reduced to $30 \mathrm{mbar}$ and further advancement of the pipette was made in 2-3 $\mu$ m steps. A sudden decrease of the current pulse typically indicated contact with a cell and the positive pressure was released for giga seal formation. After successful formation of a giga seal at a holding potential of $-70 \mathrm{mV}$, the pipette capacitance was compensated and the cell membrane was ruptured with short negative pressure pulses. Whole-cell recordings were then carried out in the current clamp mode following bridge balancing. Only cells with a resting membrane potential of $\leq-50 \mathrm{mV}$ and with initial overshooting APs were included in the analysis. Recordings were discontinued once the resting potential increased above $-50 \mathrm{mV}$, or the amplitude of APs dropped below $40 \mathrm{mV}$. The layer 4 recordings were performed at depths between 350-500 $\mu \mathrm{m}$. The membrane potential was not corrected for liquid junction potentials.

In order to measure light evoked potentials, a small LED lamp was positioned $10 \mathrm{~cm}$ from the contralateral eye. The duration and onset of light pulses were controlled with the pClamp recording 
software and were monitored with a photodiode that was placed in close proximity to the LED lamp. The light pulses had a duration of 2 ms and were applied at a fixed intensity.

The following solutions were used for experiments:

Intracellular solution (based on (Crochet and Petersen, 2006)):

$135 \mathrm{mM}$ potassium gluconate, $4 \mathrm{mM} \mathrm{KCl}, 10 \mathrm{mM}$ HEPES, 10 mM Na 2 Phosphocreatine, 4 mM Mg-ATP, $0.3 \mathrm{mM} \mathrm{Na-GTP}$ (solution adjusted to $\mathrm{pH} 7.3$ with $\mathrm{KOH} ;+2 \mathrm{mg} / \mathrm{ml}$ biocytin).

Extracellular solution ("Lockes solution")

$154 \mathrm{mM} \mathrm{NaCl}, 5.58 \mathrm{mM} \mathrm{KCl}, 0.84 \mathrm{mM} \mathrm{NaH}_{2} \mathrm{PO}_{4}, 2.14 \mathrm{mM} \mathrm{Na}_{2} \mathrm{HPO}_{4}$ and $10 \mathrm{mM}$ glucose (solution adjusted to $\mathrm{pH} 7$ with $\mathrm{NaOH}$ )

\subsection{Immunocytochemistry}

Immunocytochemistry of cultured autaptic neurons at DIV14 was carried out based on published protocols (Nair et al., 2013; Ripamonti et al., 2017). In brief, cells were chemically fixed in an ice cold phosphate buffered saline (PBS) solution containing 4\% PFA and 4\% sucrose for 30-40 min. This step was then followed by a 30 min incubation of cells in a PBS-based blocking solution containing $0.3 \%$ Triton-X, $10 \%$ goat serum and $0.1 \%$ fish skin gelatin. Next, neurons were incubated in primary antibodydiluted blocking solution for $2 \mathrm{~h}$ at room temperature and washed three times using PBS, before blocking solution supplemented with secondary antibodies was added to cells. Following an additional washing of cells with PBS, coverslips were mounted and stored overnight at $4^{\circ} \mathrm{C}$.

The following antibodies were used for experiments (Tab.1)

Table 3: List of primary antibodies used for immunocytochemistry.

\begin{tabular}{lllll}
\hline Antibody & Species & Dilution & Company/Manufacturer & Cat. Number \\
\hline Bassoon, 219E monoclonal & Mouse & $1: 1000$ & Synaptic Systems & 141021 \\
\hline MAP2, polyclonal & Rabbit & $1: 1000$ & Millipore & AB5622 \\
\hline Mun13-1, polyclonal & Rabbit & $1: 500$ & Synaptic Systems & 126103 \\
\hline Shank-2, antiserum & Guinea Pig & $1: 1000$ & Synaptic Systems & 162204 \\
\hline Synapsin 1, monoclonal & Mouse & $1: 1000$ & Synaptic Systems & 106021 \\
\hline VGLUT1, polyclonal & Guinea Pig & $1: 1000$ & Synaptic Systems & 135304
\end{tabular}

The antibodies were stored as glycerol stocks ( $1: 2$ dilution) at $-20^{\circ} \mathrm{C}$ 
Table 4: List of secondary antibodies used for immunocytochemistry

\begin{tabular}{llll}
\hline Antibody & Fluorophore & Dilution & Company/Manufacturer \\
\hline Goat $\alpha$ Mouse & 555,633 & $1: 1000$ & Invitrogen \\
\hline Goat $\alpha$ Guinea Pig & 488,633 & $1: 1000$ & Invitrogen \\
\hline Goat $\alpha$ Rabbit & 488,555 & $1: 1000$ & Invitrogen
\end{tabular}

The antibodies were stored as glycerol stocks (1:2 dilution) at $-20^{\circ} \mathrm{C}$

\subsubsection{Imaging}

Microscopy of fluorescently stained neurons was performed using an inverted SP2 Leica confocal microscope equipped with a $63 x$ objective (NA 1.4). For the quantification of synapsin positive puncta, $z$-stack images were taken at $1024 * 1024$ resolution at steps of $0.12 \mu \mathrm{m}$. These image-stacks were then projected at maximal intensity using ImageJ and the number of structures was assessed based on a previously published procedure (Ripamonti et al., 2017; Varoqueaux et al., 2002). In brief, the mean intensity of background fluorescence was removed from projected z-stacks using a custom written ImageJ plugin (provided by Ali Shaib and Ute Becherer) and a manually fixed intensity threshold was applied to synapsin signals of control- and experimental groups followed by watershed filtering and an analysis of the number of particles $\geq 0.17 \mu \mathrm{m}$. In order to quantify the fraction of synapsin puncta that contained postsynaptic shank2 signals, regions of interests (ROIs) at sizes of $50 \times 10 \mu \mathrm{m}$ were selected from single z-plane images and co-localization was studied by intensity thresholding combined with particle size discrimination. In order to study the intensity level of Munc13-1 fluorescence signals in VGLUT1/Bassoon positive puncta, single z-plane images were acquired at $512 \times 512$ resolution with a digital zoom factor of 4 . ROIs of $20 \times 5 \mu \mathrm{m}$ were selected from images and the mean intensity of Munc131 fluorescence was measured in puncta that contained both VGLUT1- and Bassoon signals, after the mean intensity of the background was subtracted.

\subsection{Immunohistochemistry}

Following in-vivo whole cell recordings, a selected number of mice were deeply anaesthetized using ketamine and whole animal PFA perfusion was carried out based on a previously published protocol (Gage et al., 2012). After sucrose cryoprotection, PFA-fixed brain tissue was subjected to cryosectioning and 40-200 $\mu \mathrm{m}$ thick slices were prepared. These were then incubated for 3 hours at room temperature in PBS solution containing 0.3\% Triton, streptavidin conjugated Alexa 555 (diluted at 1:1000) and DAPI $(\sim 1 \mu \mathrm{M})$. In a last step, brain sections were washed three times with PBS and mounted unto glass 
coverslips for microscopy. For immunohistochemistry of brains from SERTcre/Ai32 mice, PFA-fixed tissue was directly mounted unto coverslips after sectioning using a DAPI-supplemented mounting solution. Low magnification images were acquired using a Leica MZ16 binocular and dendritic structures were visualized with a $63 x$ objective (NA 1.4) using a SP2 confocal microscope.

\subsection{Data Analysis}

Electrophysiological data was analyzed using axograph (AxoGraph Scientific) and Matlab (Mathworks, Inc.) software. Statistical analysis was carried out using GraphPad Prism 5 (GraphPad Software, Inc.). A Kolmogoric-Smirnov test was applied to test Gaussian distribution of data. For normally distributed data, significance was determined using the two-tailed student's t-test, or one-way ANOVA followed by post hoc Bonferroni tests. For non-parametric data sets, statistical significance was determined using the Mann-Whitney test, or the Kruskal Wallis test followed by Dunns Multiple Comparison tests. All data are given as mean and standard error of mean (SEM). 


\section{Results}

\subsection{Electrophysiological analysis of the relevance of mSYD1s and Mover in regulating transmitter release in cultured neurons}

The release of transmitter is orchestrated by a large number of proteins. Two such proteins, mSYD1A and Mover, have recently been identified as novel regulators of the release machinery (Korber et al., 2015; Wentzel et al., 2013). Deletion of mSYD1A was shown to lead to strong reductions ( $50 \%)$ in the number of docked vesicles and in the frequency of spontaneously occurring mEPSCs in hippocampal slice preparations (Wentzel et al., 2013). Knock-down of Mover, in contrast, was reported to lead to more subtle changes, namely small increases in AP-evoked EPSC amplitudes and enhanced short-term synaptic depression in the Calyx of Held synapse (Korber et al., 2015). To study the contribution of these proteins in transmitter release in greater detail and to further assess their potential involvement during SV priming, I functionally dissected the consequences of the respective KOs for synaptic transmission in cultured autaptic hippocampal cells. These cells have successfully been applied in many studies for the functional analysis of synaptic proteins, and parameters of synaptic transmission, such as RRP size, vesicular release probability and frequency of MEPSCs, can be studied in isolated neurons in an easily accessible fashion (Bekkers and Stevens, 1991; Burgalossi et al., 2012; Rosenmund and Stevens, 1996).

\subsubsection{Synaptic transmission is not perturbed in cultured mSYD1A- deficient neurons}

Genetic deletion of mSYDA1 does not result in any obvious behavioral abnormalities, and genotyping of mice yielded reliable results as confirmed by western blot analysis (Wentzel et al., 2013) (Fig.4 A). A battery of electrophysiological protocols was carried out to study various aspects of synaptic transmission in cultured hippocampal cells from mSYD1A KO- and wild type (WT) littermate control mice. Included in the analysis was the exogenous application of hyperosmotic sucrose solution, which is a strong stimulus that depletes the entire pool of primed vesicles and thus leads to a pronounced EPSC whose charge is commonly used to assess the RRP size (Rosenmund and Stevens, 1996). Based on previous findings that the RRP size correlates with the number of vesicles docked to the plasma membrane, I speculated that the strong docking deficit reported for mSYD1 KO neurons would become manifest as a pronounced reduction in sucrose evoked EPSC charges (Imig et al., 2014; Murthy et al., 2001; Schikorski and Stevens, 2001; Wentzel et al., 2013). Surprisingly, however, I could not detect any statistically significant differences in AP- and sucrose evoked responses between mSYD1A KO- and WT cells (Fig.4 D-E). Correspondingly, no differences in the vesicular release probability, which is calculated 
by dividing the charge of AP-triggered EPSCs by the charge of sucrose evoked responses, could be detected (Fig 4 F).

I next tested whether deletion of mSYD1A leads to a similar reduction in the frequency of spontaneously occurring mEPSCs as reported in the literature for synaptic transmission in the CA1 region of acute slices prepared from the hippocampus of mSYD1A KO animals (Wentzel et al., 2013). Isolated mEPSCs reflect the spontaneous fusion of single SVs with the plasma membrane and their amplitude - commonly referred to as the quantal size - gives a measure of the postsynaptic current that is evoked by such fusion events (Del Castillo and Katz, 1955; Heuser et al., 1979). Changes in the loading of SVs with transmitter molecules, or in the abundance and composition of postsynaptic receptor complexes may cause differences in the amplitude of miniature currents (Edwards, 2007). The frequency of mEPSCs, on the other hand, is strongly dependent on presynaptic parameters including, but not limited to, the $\mathrm{Ca}^{2+}$ sensitivity and the fusogenicity state of SVs (Basu et al., 2007; Kaeser and Regehr, 2014). Interestingly, recent studies reported that spontaneously fusing vesicles may belong to a pool of SVs that is distinct from the vesicle population involved in AP-evoked release of transmitter (Crawford et al., 2017; Kavalali, 2015; Truckenbrodt and Rizzoli, 2014). Accordingly, one possible scenario in the light of the findings of a reduced mEPSC frequency in hippocampal slices of SYD1A KO mice could be that SYD1A selectively regulates the release of transmitter from spontaneously fusing vesicles. Strikingly, however, I could not detect any statistically significant differences between KO- and WT cells in the amplitude, frequency or time course of mEPSCs measured in the presence of 300 nM TTX (Fig.4 C, G). Taken together, these results indicate that mSYD1A is not involved in regulating AP-evoked or spontaneous transmitter release in cultured hippocampal neurons at basic levels of presynaptic activity. Furthermore, the priming of vesicles was found to be completely intact as assessed by measurements of sucrose evoked responses.

To further test, whether mSYD1A modulates the release machinery or the priming process during enhanced levels of presynaptic activity, I applied trains of APs at frequencies of 2, 10 and $40 \mathrm{~Hz}$ and studied STP characteristics (Fig4 I-K). The paired-pulse ratio (PPR) between two consecutive EPSCs in the beginning of AP trains typically correlates with the initial release probability and was found to not be changed in SYD1A deficient cells, in accordance to previously published results (Wentzel et al., 2013). The steady-state EPSC amplitude during AP stimulation was used as measure for the degree of shortterm synaptic depression. Defects in the activity-dependent priming process often lead to a more pronounced reduction of EPSC amplitudes during stimulation, while less-prominent depression can be caused by an enhanced priming rate (Shin et al., 2010). Interestingly, no difference in the degree of short-term synaptic depression was found between $\mathrm{KO}$ - and control cells at the tested AP frequencies, indicating that the SV priming process is not compromised in absence of SYD1A proteins. 

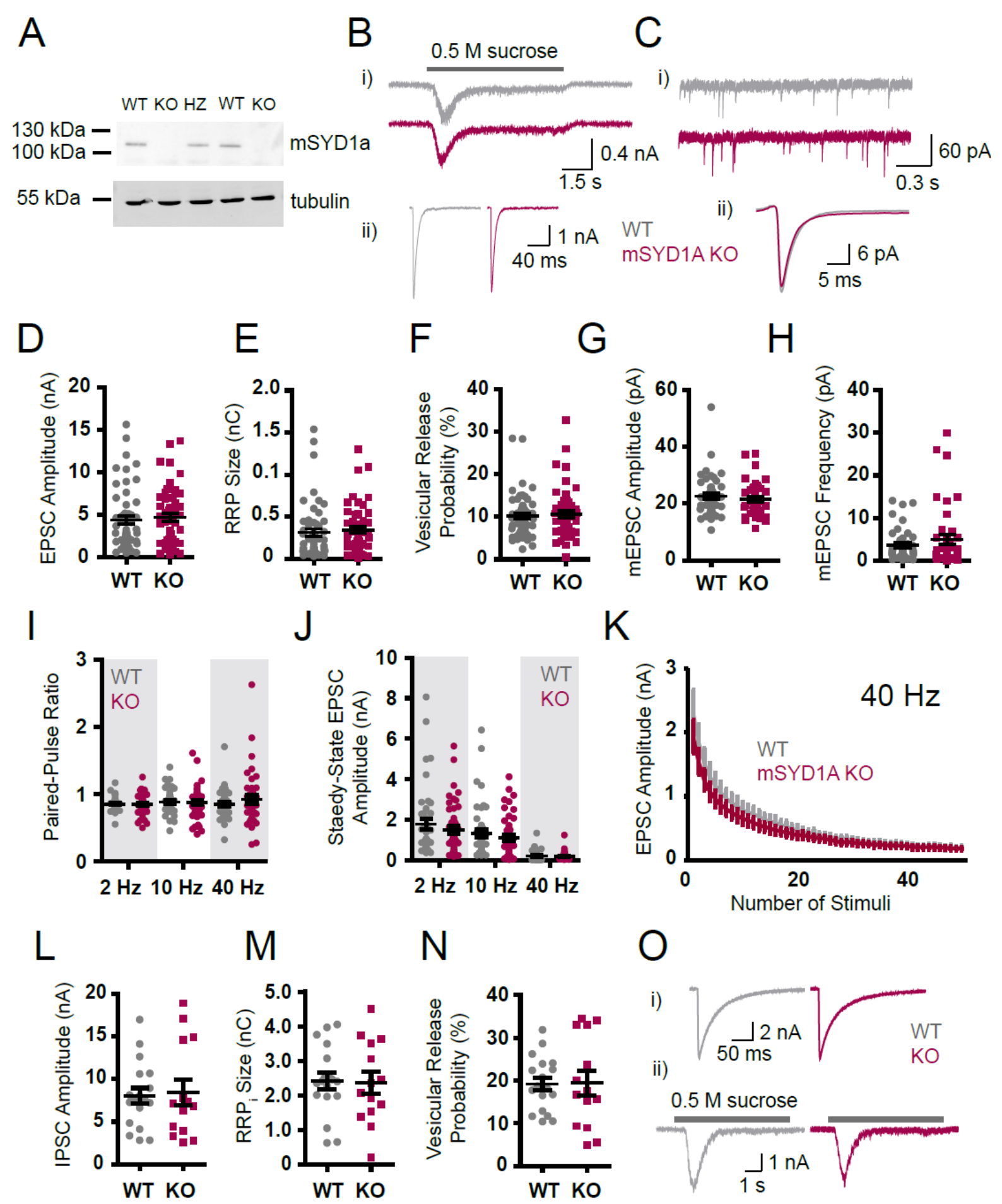

Figure 4: Synaptic transmission is not altered in cultured mSYD1A-deficient neurons. (A) Western blot analysis of brain homogenates from mice with indicated genotypes. $(W T=$ wild type, $H Z=$ heterozygous, $K O=$ knock-out; the primary antibody against mSYD1A was kindly provided by Corinna Wentzel) (B) Representative recordings of EPSCS triggered in cultured autaptic neurons by hyperosmotic sucrose solution (i) or single APs (ii), respectively. (C) Example traces for spontaneously occurring mEPSCs (i) and averaged responses ( $n=42$ and 43 cells) (ii). (D) Mean amplitude of EPSCs evoked by single APs ( $n=57$ and 54 cells). (E) RRP size determined by applying $0.5 \mathrm{M}$ sucrose solution and measuring the resultant charge ( $n=55$ and 50 cells). (F) Mean vesicular release probability calculated by dividing the charge of AP-evoked EPSCs by the charge of sucrose evoked responses ( $n=55$ and 50 cells). (G) and (H) Mean amplitude and frequency of mEPSCs measured in presence of $300 \mathrm{nM}$ TTX for $100 \mathrm{~s}$ ( $n=43$ and 42 cells). (I) Paired-pulse ratio calculated by dividing the second EPSC by the first EPSC response during AP trains at the indicated frequencies ( $n=41-45$ cells). (J) Averaged steady-state EPSC responses during trains of APs at indicated 
frequencies ( $n=41-45$ cells). (K) Mean EPSC responses during a $40 \mathrm{~Hz}$ AP train ( $n=41$ and 45 cells) (L) - (N) Mean values obtained for measurements in cultured hippocampal interneurons in correspondence to measurements depicted in $D-F(n=18$ and 14 cells). (O) Representative traces for AP-and sucrose triggered IPSCs. (Bars in plots depict mean and SEM. $N=4$ cultures).

Taken together, neither an essential, nor a modulatory role of mSYD1A in excitatory synaptic transmission could be established in cultured, autaptic hippocampal neurons in the present study.

In a second set of experiments, I studied the consequences of mSYD1A deletion on basic inhibitory synaptic transmission in cultured, autaptic hippocampal interneurons. The role of mSYD1A in inhibitory synaptic transmission has not yet been addressed in the literature and there are no reports available that describe its expression in interneurons, possibly because of the lack of antibodies that are suitable for immunocytochemistry against mSYD1A.

In correspondence to findings in excitatory neurons, no differences in the amplitude of AP- or sucrose triggered responses, or in the vesicular release probability could be detected (Fig. 4 L-O). Accordingly, mSYD1A is neither essential for excitatory nor for inhibitory synaptic transmission in cultured, autaptic hippocampal neurons.

\subsubsection{Synaptic transmission is not altered in cultured mSYD1A,B DKO neurons}

One scenario that may have caused the discrepancy between previously published results and the data described above is that another mSYD1 isoform compensates the lack of mSYD1A. To test this, I studied synaptic transmission in cultured neurons from SYD1A,B DKO mice. In contrast to mSYD1A, no study has yet been conducted to detect mSYD1B at the protein level and apart from a predicted protein domain structure that resembles features of mSYD1A, no other information is available for this isoform (Wentzel et al., 2013).

The single KO of SYD1B, as well as the DKO of SYD1A and SYD1B do not cause any obvious harmful phenotype in mice (own observations and personal communication with Corinna Wentzel). Furthermore, EPSCS evoked by single APs or sucrose solution, or the amplitude and frequency of spontaneously occurring mEPSCs did not reveal any differences between DKO- and doubleheterozygous- (mSYD1 A +/-, mSYD1 B +/-), or between single mSYD1 A- and mSYD1 B KO cells obtained from mice of the same litter (Fig.5 A-C, F, G; Supplementary Fig. 1). In further similarity to the analysis of single SYD1A KO neurons, no changes in STP characteristics were associated with removal of both isoforms, as revealed by the analysis of PPRs, the steady-state EPSC amplitude and recovery of EPSCs $2 \mathrm{~s}$ 
after strong presynaptic stimulation at $100 \mathrm{~Hz}$ (Fig.5 E, H-J). Accordingly, a compensatory mechanism for a loss of mSYD1A mediated by the only other known mSYD1 isoform, mSYD1B, was not relevant during my experiments, and a previously published role of mSYD1A in regulating transmitter release could not be confirmed by the results of this study using autaptic cultured cells.

A

i)

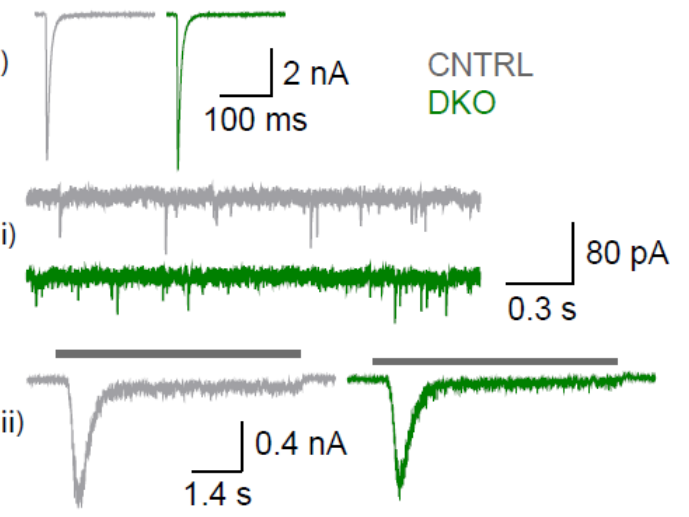

$\mathrm{D}$

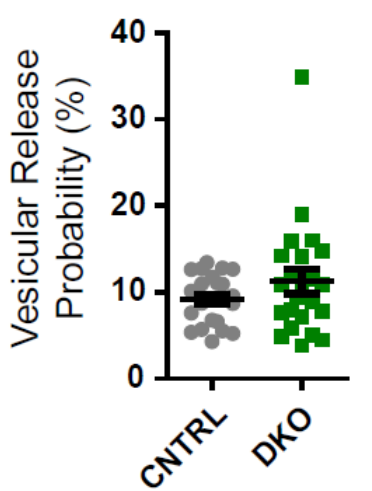

$\mathrm{H}$

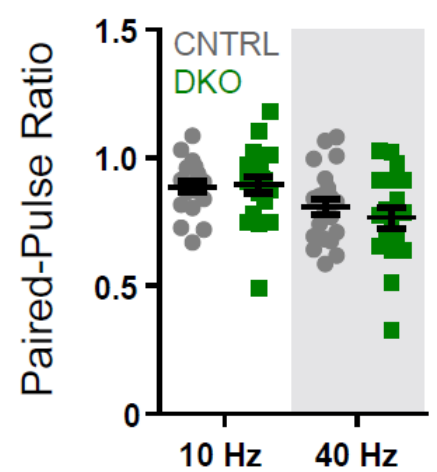

$E$

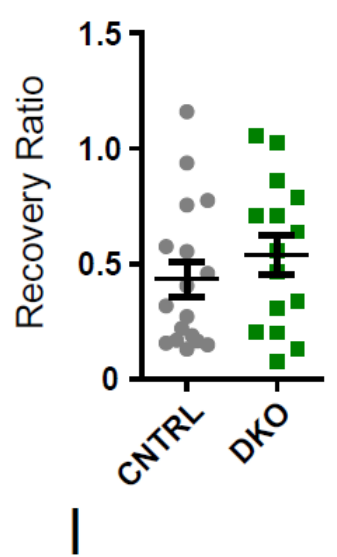

$\mathrm{B}$

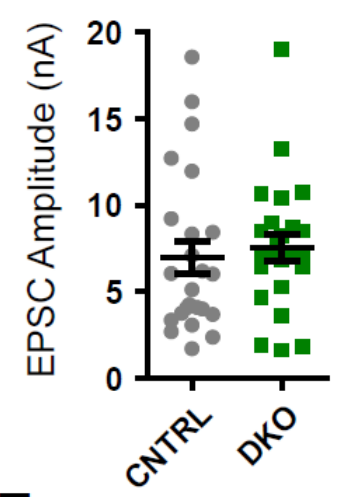

F

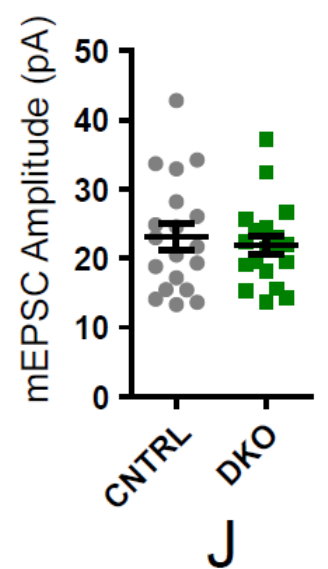

C

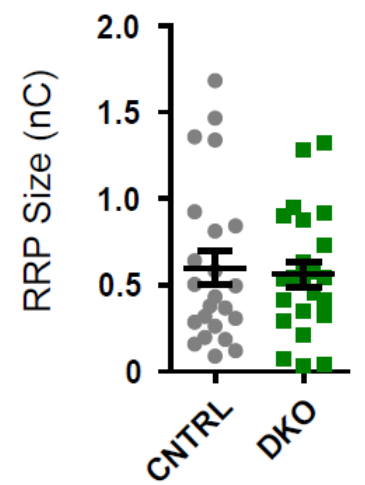

G

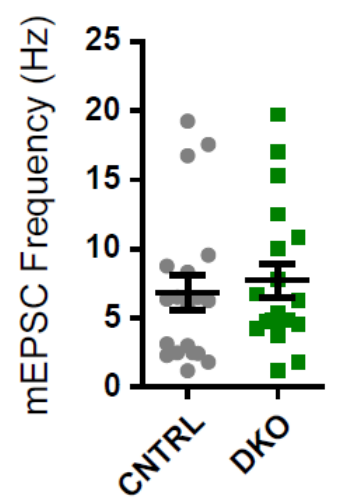

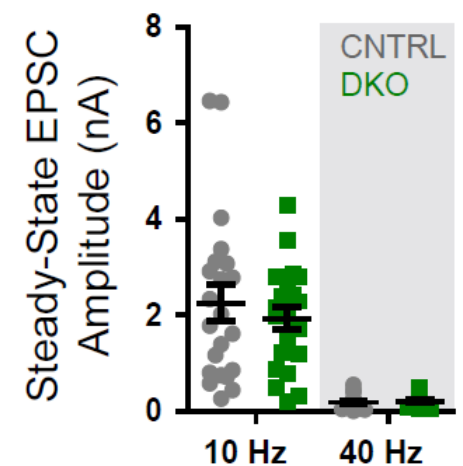

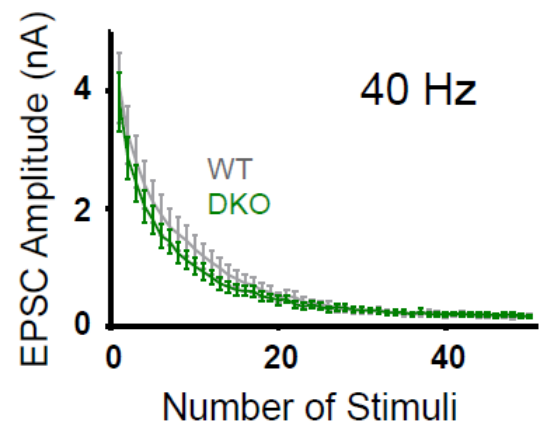

Figure 5: Synaptic transmission is not altered in cultured mSYD1-A,B DKO neurons. (A) Representative traces of APevoked EPSCS (i), spontaneously occurring MEPSCS (ii) and sucrose-triggered responses in cultured autaptic neurons (iii). (B) Mean EPSC amplitude ( $n=24$ cells each). (C) RRP size determined by measuring the charge transfer during application of sucrose solution ( $n=23$ cells each). (D) Mean vesicular release probability calculated by dividing the charge of AP-evoked EPSCs by the charge of sucrose evoked responses ( $n=23$ cells each). (E) EPSC recovery ratio calculated by dividing the amplitude of a single EPSC 2s after a 10s AP train at $100 \mathrm{~Hz}$ by the EPSC response before the train ( $n=17$ and 15 cells). (F) and (G) Mean amplitude and frequency of mEPSCs measured in presence of 300 nM TTX for 100s ( $n=19$ cells each). (H) Paired-pulse ratio calculated by dividing the second EPSC by the first EPSC response during AP trains at the indicated frequencies ( $n=19-22$ cells). (I) Averaged steady-state EPSC responses 
during trains of APs at indicated frequencies ( $n=19-22$ cells). (J) Mean EPSC responses during a train of APs at a frequency of $40 \mathrm{~Hz}(n=21$ and 19 cells). (Bars in plots depict mean and SEM. $N=1$ culture)

\subsubsection{Deletion of Mover does not lead to changes in synaptic transmission in cultured hippocampal neurons}

I next tested the relevance of the presynaptic protein Mover in regulating synaptic transmission in-vitro (Ahmed et al., 2013; Korber et al., 2015; Kremer et al., 2007). For this purpose, I prepared cultured autaptic cells from the hippocampus of Mover KO- and WT littermate control mice. The genotyping of mice and validation of Mover deletion in autaptic cultures by immunocytochemistry were carried out in collaboration with Julio Viotti (data not shown). Based on previous findings from knock-down experiments in the Calyx of Held synapse, in which Mover was reported to act as a negative regulator of the vesicular release probability by decreasing the apparent sensitivity of release to $\mathrm{Ca}^{2+}, \mathrm{I}$ anticipated to measure an increase in the amplitude of AP-evoked EPSCs and an increase in the vesicular release probability, leading to enhanced short-term synaptic depression (Korber et al., 2015).

Surprisingly, however, I could not detect any differences in AP-triggered EPSCs between KO- and control cells (Fig.6 A, B). Furthermore, the number of primed vesicles was not different between mutant and control neurons, as revealed by measuring the size of the RRP via the application of hyperosmotic sucrose solution (Fig. 6 C). Correspondingly, calculation of the vesicular release probability did not yield significantly different values between the two groups (Fig.6 D).

I next tested, whether Mover may be involved in regulating the frequency of mEPSCs. In accordance to previous findings from Mover knock-down experiments in the Calyx of Held synapse, I could not detect any statistically significant differences in the amplitude and frequency of mEPSCs between mutant- and control neurons (Fig.6 E, F). Since responses of cultured neurons to exogenously applied glutamate also revealed no differences, I concluded that basic pre- and postsynaptic function in cultured neurons was not compromised upon deletion of Mover (Fig.6 K).

To further test, whether deletion of Mover results in enhanced synaptic depression, as previously reported for knock-down experiments in the Calyx of Held synapse, I applied trains of APs to cultured neurons at frequencies of 10 and $40 \mathrm{~Hz}$. The amplitudes of EPSCs did not depress more prominently than WT control neurons to the AP trains, and the recovery of EPSC responses after high-frequency stimulation (HFS) remained unchanged in absence of Mover (Fig.6 G-J).

Based on these results, a role of Mover in regulating synaptic transmission in cultured hippocampal neurons could not be deduced, in contrast to published results using a knock-down approach in the Calyx of Held synapse. 


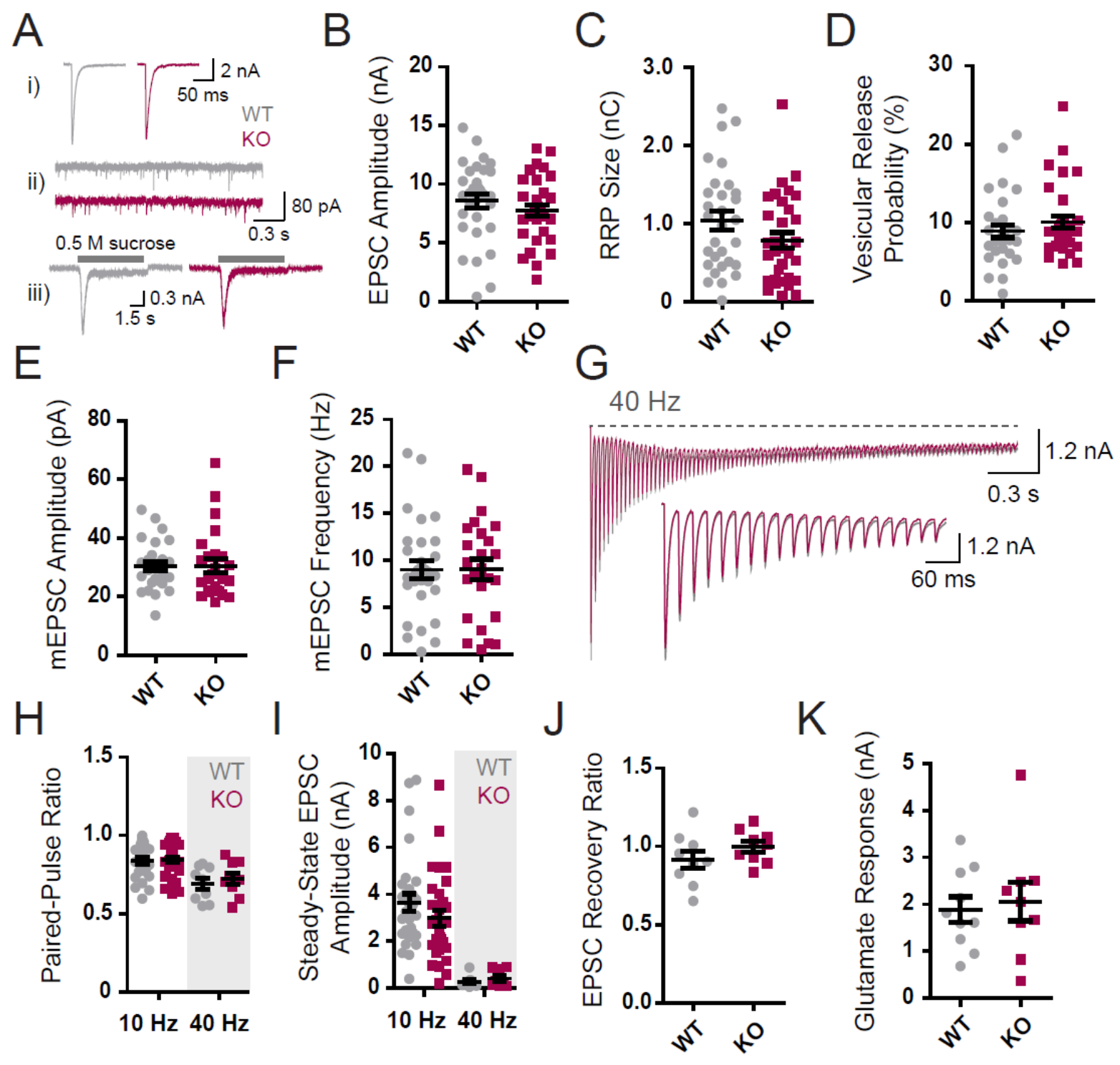

Figure 6: Synaptic transmission is not altered in cultured neurons from Mover KO mice. (A) Sample traces of APevoked EPSCs (i) spontaneously occurring mEPSCs (ii) and sucrose-evoked EPSCs in cultured autaptic neurons. (B) Mean amplitude of AP-triggered EPSCs ( $n=31$ and 32 cells). (C) RRP charge measured in presence of $0.5 \mathrm{M}$ sucrose solution ( $n=30$ and 32 cells) (D) Vesicular release probability calculated by dividing the charge of AP-evoked EPSCS by the charge of sucrose evoked responses ( $n=30$ and 32 cells). (E) and (F) Mean amplitude and frequency of mEPSCS measured in presence of $300 \mathrm{nM}$ TTX for 100s. ( $n=29$ and 25 cells). (G) Averaged EPSC responses during a $40 \mathrm{~Hz}$ AP train ( $n=9$ cells each) $(H)$ and (I) Paired-pulse ratio and steady-state EPSC responses during trains of APs at indicated frequencies ( $n=29$ and 30 cells for $10 \mathrm{~Hz}, n=9$ cells each for $40 \mathrm{~Hz}$ stimulation). (J) Recovery of EPSC amplitudes given as the ratio between the EPSC amplitude after and before a train of $100 \mathrm{APs}$ at $40 \mathrm{~Hz}(n=9$ cells each). (K) Mean responses evoked by application of a $100 \mu \mathrm{M}$ glutamate solution ( $n=10$ and 9 cells). (Bars in plots depict mean and SEM. N = 1-2 cultures). 


\subsection{Electrophysiological dissection of the functional interplay between CAPSs and Munc13s during SV priming}

Proteins of the Munc13 and CAPS families are absolutely required for synaptic transmission and deletion of any of the two factors leads to severe perturbations in transmitter release (Jockusch et al., 2007; Varoqueaux et al., 2002). In contrast to Munc13-deficient cells, however, transmitter release can transiently be restored in CAPS DKO neurons when intracellular calcium ([Ca $\left.\left.{ }^{2+}\right] \mathrm{i}\right)$ has increased after strong presynaptic stimulation, or by phorbol esters, which chemically mimic DAG (Jockusch et al., 2007). Since elevated levels of [ $\left.\mathrm{Ca}^{2+}\right]$ i and application of phorbol esters have been shown to modify and enhance the priming activity of Munc13s, it was speculated that the functional requirement of CAPSs could be by-passed by an activity dependent priming mechanism mediated by Munc13s (Jockusch et al., 2007; Junge et al., 2004; Rhee et al., 2002; Shin et al., 2010). To test this hypothesis and to address a potential, functional interplay between CAPSs and Munc13s during priming, I performed an in-depth analysis of the residual priming activity present in CAPS deficient neurons.

\subsubsection{PDBu partially rescues the priming deficit in CAPS DKO neurons}

Deletion of CAPSs results in a $68 \%$ decrease in the amplitude of AP-evoked EPSCs in cultured hippocampal neurons and a fraction of $39 \%$ of cells fails to release transmitter when single APs are applied (Jockusch et al., 2007). The deficit in AP-evoked responses is rescued upon application of PDBu to cultured DKO neurons resulting in EPSC amplitudes comparable in their sizes to evoked responses in untreated control cells (Fig.7 A) (Jockusch et al., 2007). At the present time it is unknown, whether the potentiation of AP-triggered EPSCs by PDBu is accompanied by an increase in the RRP size in CAPS DKO cells. In order to address this, I studied sucrose evoked EPSCs in the presence- and absence of PDBu in cultured CAPS DKO neurons. Strikingly, application of PDBu led to a statistically significant increase in the size of the RRP in CAPS DKO neurons (Fig.7 B, D), thereby reflecting a possible increase in the residual priming activity.

Another way to detect a potential increase in priming activity, is to subject neurons to trains of APs and to study the steady-state of EPSC amplitudes during the trains. An enhanced priming activity may become manifest as an increase in the steady-state level, while changes in the release probability would lead to an altered PPR (Korber et al., 2015; Shin et al., 2010). In order to study these parameters in untreated- and PDBu treated DKO cells, I first subjected cells to strong HFS consisting of 100 APs at 40 $\mathrm{Hz}$ to generate a pool of newly primed vesicles, and subsequently applied trains of APs at varying frequencies (Fig.7 E). Treatment of cells by PDBu led to a pronounced increase in the normalized steady- 
state EPSC amplitude of DKO cells at frequencies of 2 and $5 \mathrm{~Hz}$, while the PPR was not different between the two groups (Fig.7 F, G). This finding may indicate that the vesicular release probability after HFS is similar in both conditions, while the overall priming activity may be higher in treated cells (see discussion for alternative interpretations). Despite this promoting effect of PDBu on sustained release, however, normalized steady state EPSC amplitudes and PRRs of treated DKO cells did not reach the levels of untreated control cells.

A
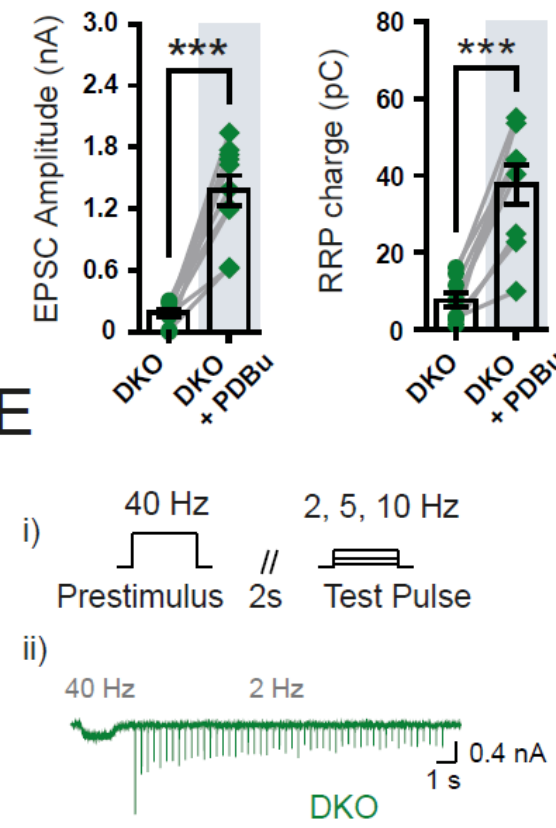

$\mathrm{B}$
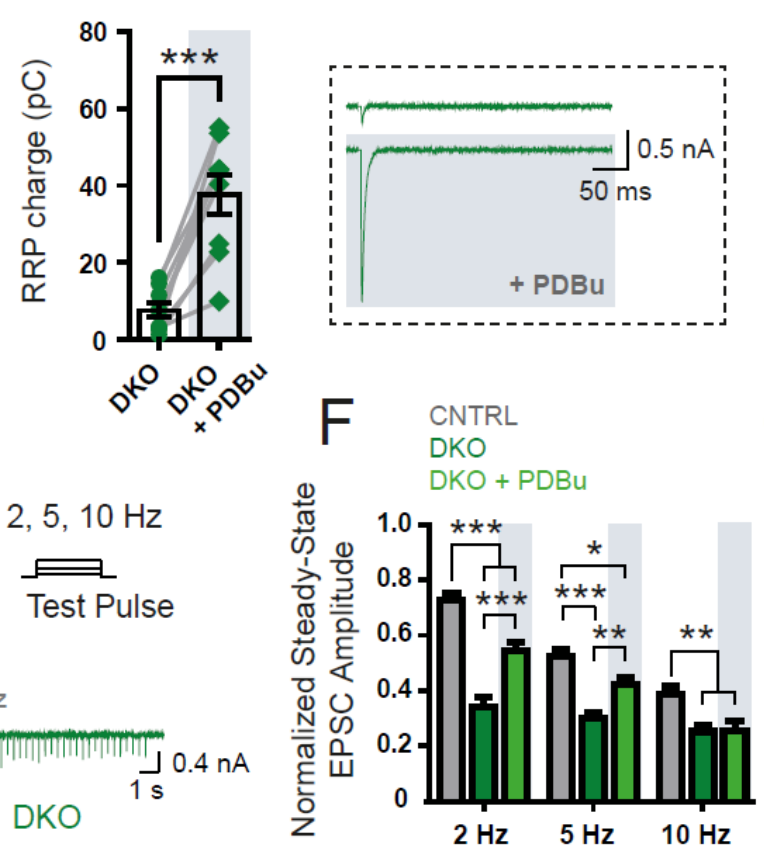
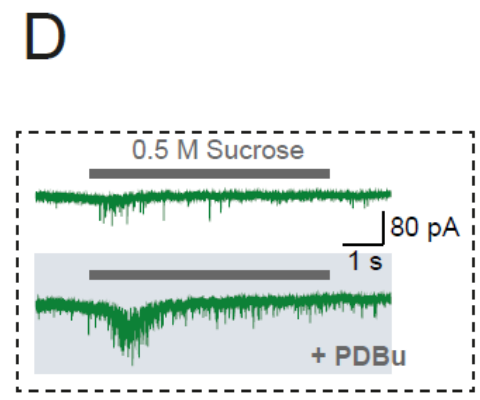

G CNTRL

DKO

$\mathrm{DKO}+\mathrm{PDBu}$

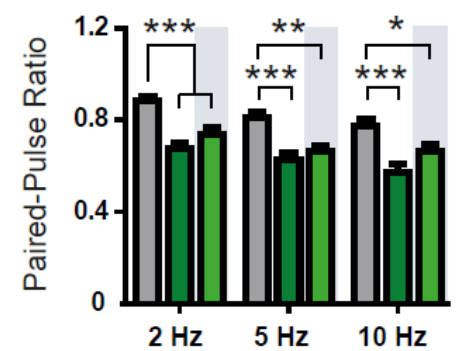

Figure 7: PDBu partially rescues transmitter release in CAPS deficient neurons. (A) AP- and (B) sucrose triggered EPSC amplitudes measured in cultured neurons of CAPS DKO mice in absence and presence of $3 \mu \mathrm{m} P D B U$ ( $N=10$ and 11 cells per genotype; two-tailed t-test; $P<0.0001)$. (C) and (D) representative traces of recordings in $(A)$ and $(B)(E)$ (i) Graphical representation of the experimental paradigm used for ( $F)$ and $(G)$, (ii) depicts a sample recording from a CAPS DKO cell. (F) and (G) Normalized steady-state EPSC amplitude and paired pulse ratio of indicated genotypes ( $n=20$-22 cells from 3 cultures; one-way ANOVA and Bonferroni's Multiple Comparison test, ${ }^{*} p<0.05 ;{ }^{* *} p<0.01$; ${ }^{* * * P}<0.001$ ). (CNTRL= littermate CAPS1 +/- and +/+ on CAPS2 KO background, Bars in scatterplots represent mean and SEM).

Taken together, these results support the previously posed hypothesis that application of PDBu results in the recruitment of an activity dependent priming process that partially compensates for the loss of CAPSs. 


\subsubsection{The $\mathrm{C} 1$ domain of Munc13 is required to functionally compensate for the loss of CAPSs}

Next, I aimed at identifying the unknown priming process that compensates for the loss of CAPSs when HFS, or PDBu is applied. Since Munc13s fulfill the criteria of being capable of enhancing their priming activity upon binding of the second messengers $\mathrm{Ca}^{2+}$, calmodulin and DAG, it has been speculated for a decade now that these proteins may execute the $\mathrm{Ca}^{2+}$ - and PDBu induced priming reaction in CAPSdeficient cells (Jockusch et al., 2007; Junge et al., 2004; Lipstein et al., 2012; Rhee et al., 2002; Shin et al., 2010).

In this study, I specifically focused my analysis on a mutation within the C1 domain of Munc13-1 (H567K) that disrupts its interaction with DAG, prevents potentiation of transmitter release by phorbol esters, reduces $\mathrm{Ca}^{2+}$-dependent Doc2b cotranslocation to the plasma membrane and enhances short-term synaptic depression (Friedrich et al., 2013; Rhee et al., 2002). Notably, it also causes a reduction in the RRP size, in contrast to other mutations in Munc13s that interfere with $\mathrm{Ca}^{2+}$-dependent $\mathrm{PIP}_{2-}$-, or calmodulin binding, thereby indicating that the C1 domain may play a more important role during priming than the other second messenger binding regions (Junge et al., 2004; Lipstein et al., 2012; Rhee et al., 2002; Shin et al., 2010).

Synaptic transmission was studied in CAPS DKO cells that additionally contained the H567K mutation in Munc13-1 and did not express Munc13-2 (in the following referred to as "CAKI"). Heterozygous CAPS1 KO mice carrying either one or two Munc13-1 WT alleles (WT/H567K, or WT/WT, respectively) on a Munc13-2/CAPS2 DKO background were used as littermate controls in the following experiments.

A basic morphological analysis of cultured CAKI- and control neurons using conventional confocal microscopy did not reveal striking differences between the two groups (Fig. 8). The number of synapses, estimated by counting the number of synapsin positive puncta in autaptic neurons, was not significantly changed in CAKI cells $(408.7 \pm 55.87)$ compared to controls (304 \pm 38.84$)$ (Fig.8 C). Since functional synapses require the apposition of pre- and postsynaptic structures, I further quantified the fraction of synapsin puncta that contained fluorescent signals for the postsynaptic protein shank-2, in order to test for a possible perturbation in the assembly of synapses. A statistically significant difference between CAKI- $(0.651 \pm 0.05)$ and control $(0.769 \pm 0.044)$ neurons could not be detected suggesting that synaptic contacts formed normally on the scale detectable by confocal microscopy (Fig.8 D). A subtle, statistically significant decrease of $16 \%$ in the intensity of synapsin puncta in CAKI cells, however, was observed, while the area of these signals was not changed when compared to control neurons (Fig.8 E-F). This subtle morphological phenotype may reflect a decrease in the density of synaptic vesicle clusters that was previously reported for CAPS- and Munc13 deficient cells, respectively (Imig et al., 2014). 

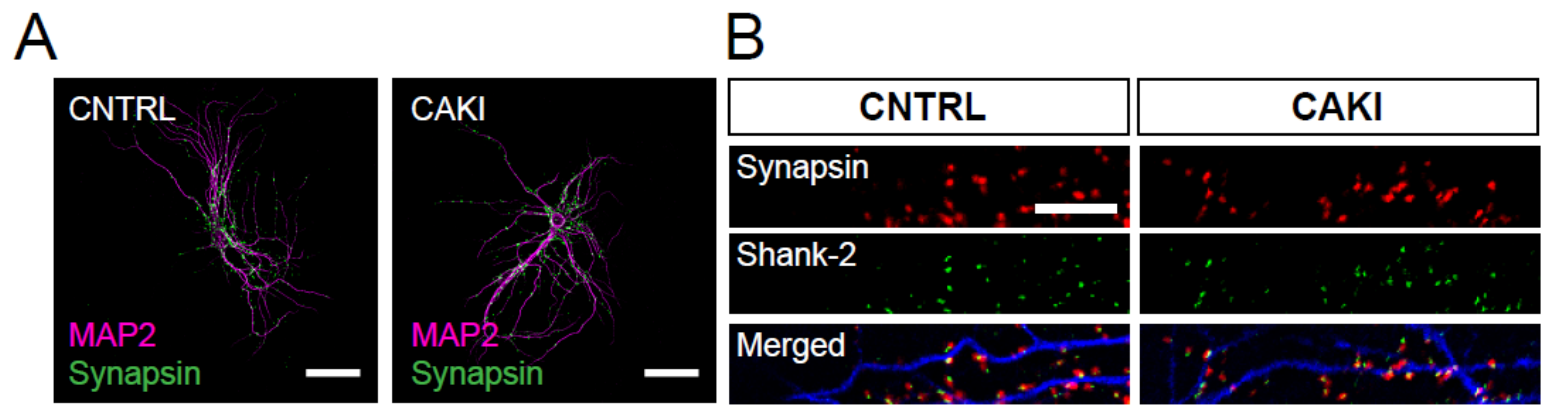

C
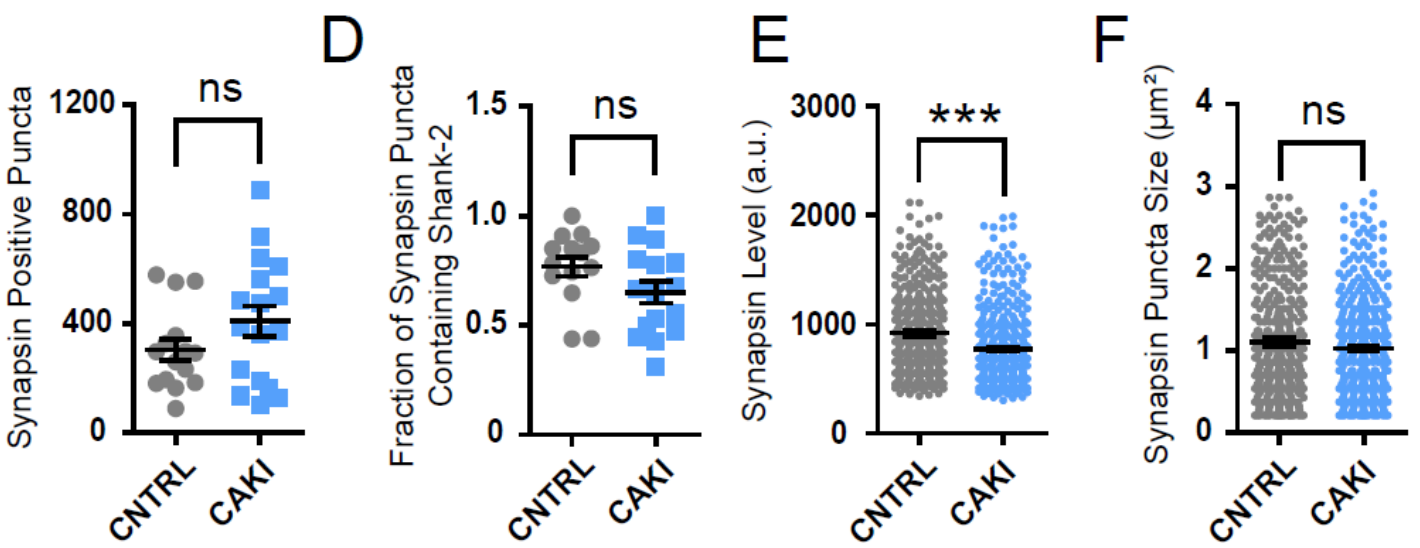

Figure 8: Morphological analysis of cultured CAKI neurons. (A) and (B) Representative confocal images of fluorescently stained autaptic neurons (scale bar, 40 and $10 \mu \mathrm{m}$ ). (C) Number of synapsin puncta per cell ( $n=15$ and 17 cells; two-tailed t-test, $P=0.1442)$. (D) Fraction of synapsin puncta containing shank-2 fluorescent signals and (E) mean intensity, as well as (F) puncta size of synapsin signals ( $n=14$ and $16,(D)$ two-tailed student's t-test, $P=$ 0.0901, (E) and (F) Mann-Whitney test, ${ }^{* * *} P<0.0001$ and $\left.P=0.1895\right)$. (CNTRL= littermate CAPS1 $+/-$, Munc13-1 WT/H567K or Munc13-1 WT/WT on CAPS2- and Munc13-2 DKO background, Bars in scatterplots represent mean and SEM, $N=3$ cultures).

I next carried out electrophysiological recordings in cultured neurons for a basic characterization of synaptic transmission in CAKI cells. Exogenous application of $100 \mu \mathrm{M}$ glutamate revealed a mild decrease ( $20 \%$ ) in postsynaptic responses that possibly reflected a slight perturbation in postsynaptic receptor composition (Fig.9 A, B). Interestingly, glutamate uncaging at spines of Munc13 DKO neurons in organotypic slices has revealed a similar decrease in postsynaptic responses, indicating that dysfunction of the presynaptic priming machinery may also lead to changes in postsynaptic composition (Sigler et al., in press).

Cultured CAKI cells further exhibited severe deficits in AP- and sucrose triggered EPSCs (Fig.9 C,D). Specifically, $43 \%$ of neurons did not exhibit EPSCs evoked by single APs ( $43 \%)$, and several cells that exhibited EPSCs in the beginning of the recordings ( 0-15 s) showed rapid rundown of responses, as reflected by the even greater fraction of cells that did not respond to subsequent sucrose pulses ( 73\%). Thus, the fraction of cells that remained silent in the beginning of recordings is similar in number to the previously reported proportion of CAPS DKO cells (39\%) that were shown to not exhibit AP-evoked release (Jockusch et al., 2007). Despite this similarity, however, the decrease in AP-triggered EPSC 
amplitudes (96\%), as well as in sucrose-evoked responses (99.5\%) of the fraction of CAKI cells that released transmitter in early phases of recordings were more pronounced than what was observed by Jockusch et al. for transmitter releasing CAPS DKO cells (68\% and $85 \%$, respectively). This indicates a more severe perturbation in the presynaptic release apparatus upon introduction of the H567K mutation in Munc13-1 in CAPS-deficient cells (Jockusch et al., 2007).

This more severe perturbation also became apparent, when the occurrence of spontaneous fusion events was quantified. The frequency of mEPSCs was reduced in CAKI neurons to approximately $5 \%$ of the control level, while amplitudes were reduced to 76\% (Fig.9 E-G). In comparison, the frequency and amplitude of mEPSCs was previously reported to be reduced to $24 \%$ and $74 \%$ of control levels in CAPSDKO cells, respectively (Jockusch et al., 2007).

Taken together, the observation of a severe reduction in AP- and sucrose evoked EPSCs, as well as in the number of spontaneously occurring mEPSCs suggest a priming deficit that is even stronger in CAKIthan in CAPS DKO cells.

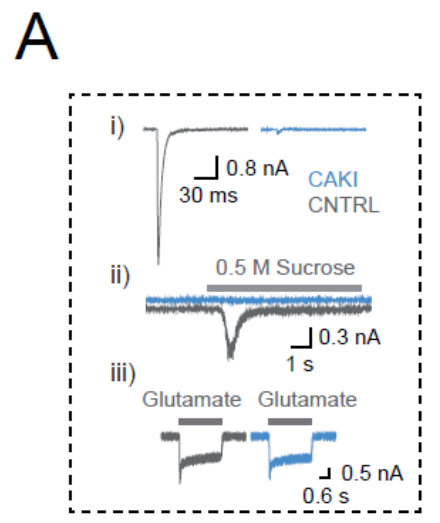

$\mathrm{E}$

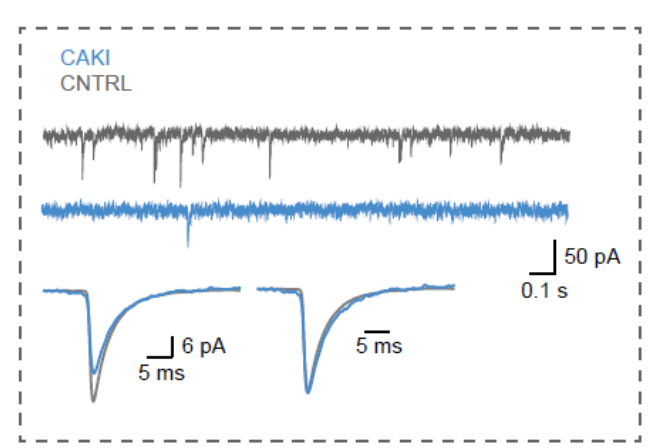

B

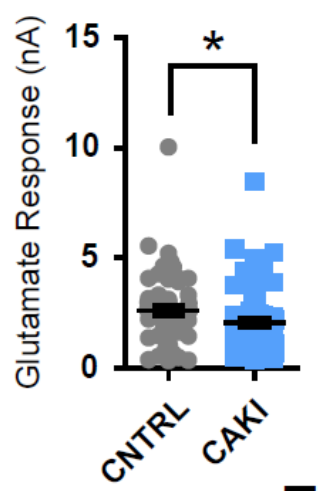

$\mathrm{F}$

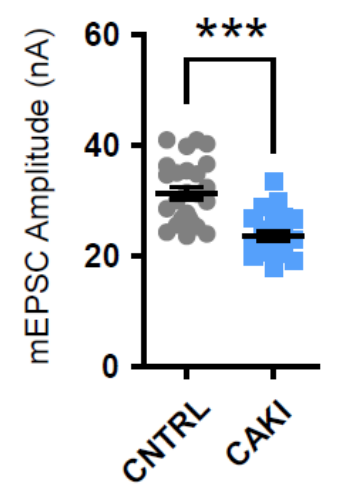

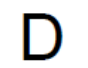
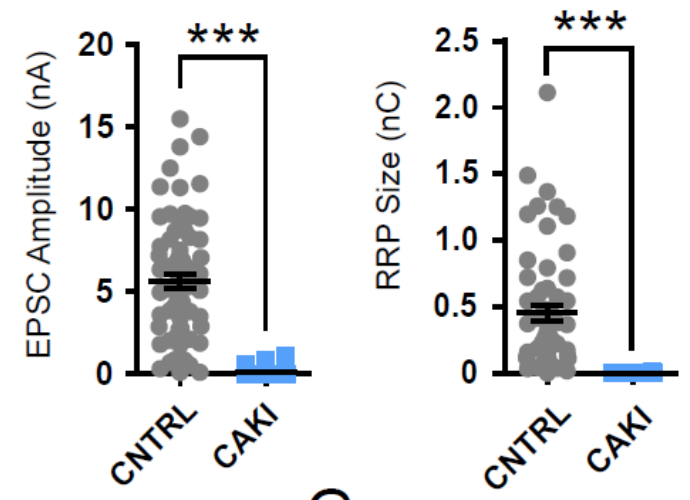

$\mathrm{G}$

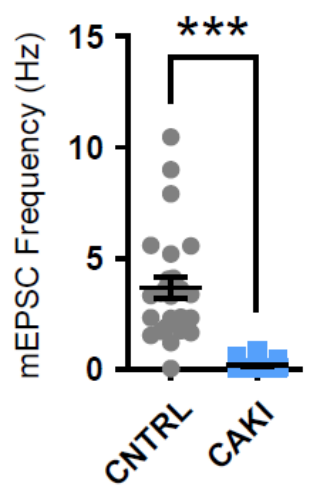

Figure 9: Transmitter release is severely perturbed in cultured CAKI neurons. (A) Example traces of (i) AP- (ii) sucroseand (iii) glutamate evoked postsynaptic currents. (B) Postsynaptic responses induced by $100 \mu \mathrm{M}$ glutamate ( $n=54$ and 57 cells, Mann-Whitney test, ${ }^{*} P=0.0255$ ). (C) EPSC Amplitude triggered by single APs ( $n=75$ and 76 cells, of these 33 CAKI cells failed to exhibit AP-triggered responses, Mann-Whitney test, $\left.{ }^{* * *}<<0.0001\right)$. (D) RRP size measured upon application of $0.5 \mathrm{M}$ sucrose solution ( $n=58$ and 56 cells, of which 41 CAKI cells did not respond to sucrose pulses; Mann-Whitney test, $\left.{ }^{* * *}>0.0001\right)$. (E) Example recordings of spontaneously occurring mEPSCs. 
The absolute and normalized averaged mEPSC response is shown below sample trace ( $n=26$ and 22 cells) (F) and (G) Amplitude and frequency of mEPSCs measured in presence of $100 \mathrm{nM}$ TTX ( $n=26$ and 22 cells, an additional number of 6 CAKI cells exhibited no mEPSCs and were excluded from analysis, Mann-Whitney test, ${ }^{* * *} P<0.0001$ ). (CNTRL= littermate CAPS1 +/-, Munc13-1 WT/H567K or Munc13-1 WT/WT on CAPS2- and Munc13-2 DKO background, Bars in scatterplots represent mean and SEM, $N=2-3$ cultures).

In order to exclude the possibility that the severe deficit in transmitter release was caused by a lowered expression level or mislocalization of mutant Munc13-1 in CAKI cells, I fluorescently stained Munc13-1 in control and mutant neurons and studied its mean intensity and localization relative to Bassoon and VGLUT1 positive puncta (Fig.10). Besides a reduction in the mean intensity of VGLUT1 signals that resembled the reduction observed for the intensity of synapsin staining described above, I did not observe any changes in the intensity of Munc13-1 signals in Bassoon-VGLUT1 positive puncta suggesting that the expression and localization was not different between Munc13-1 ${ }^{\mathrm{H} 567 \mathrm{~K}}$ and Munc13-1 ${ }^{\mathrm{WT}}$, as expected from previous results in the literature (Fig.10 A-D) (Rhee et al., 2002).
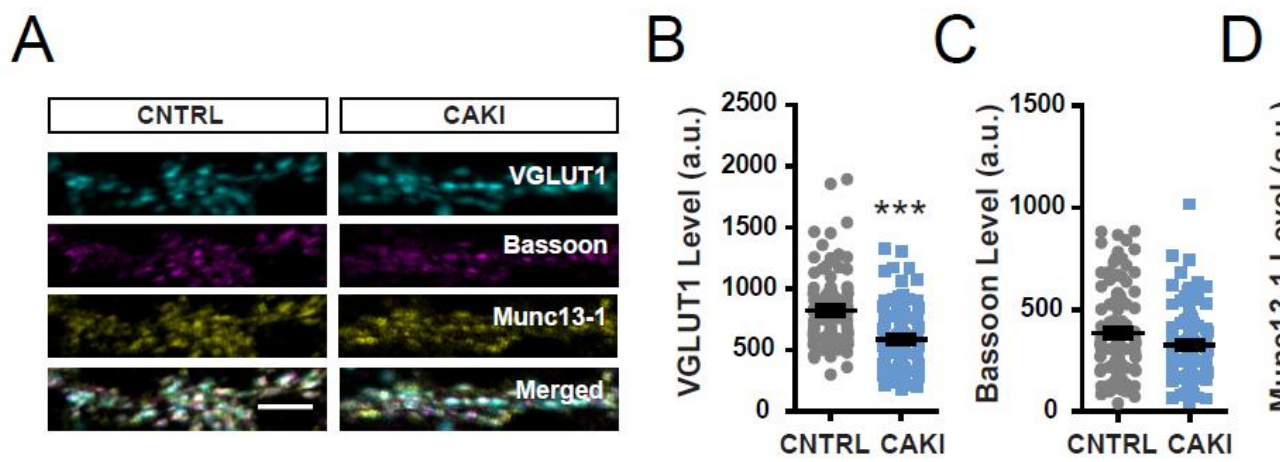

Figure 10: Expression and synaptic localization of Munc13 ${ }^{\mathrm{H} 567 \mathrm{~K}}$ is not perturbed in CAKI cells. (A) Representative confocal images of fluorescently stained autaptic neurons (scale bar, $5 \mu \mathrm{m}$ ). (B) - (D) Intensity of fluorescence signals for VGLUT1 (B), Bassoon (C) and Munc13-1 (D) in VGLUT1/Bassoon positive puncta. $(n=11$ and 10 cells, MannWhitney test, ***P<0.0001). (CNTRL= littermate CAPS1 +/-, Munc13-1 WT/H567K or Munc13-1 WT/WT on CAPS2and Munc13-2 DKO background, Bars in scatterplots represent mean and SEM, $N=1$ culture).

In a last set of experiments, I tested whether transmitter release could be rescued in CAKI cells to a similar extend as in CAPS DKO neurons by application of HFS or PDBu. For this, I applied trains of APs at frequencies of 40 and $10 \mathrm{~Hz}$, respectively, and measured the amplitude of EPSC responses in cells of the different genotypes (Fig.11). As reported previously, CAPS DKO neurons displayed tonic release during HFS and their EPSC responses facilitated during AP trains at $10 \mathrm{~Hz}$ (Fig.11 A, B, G). (Jockusch et al., 2007). In contrast, HFS failed to trigger tonic release in CAKI cells, which further showed no facilitation of their EPSC responses during the $10 \mathrm{~Hz}$ AP trains (Fig.11 C, D). Following the application of HFS or PDBu, CAPS DKO neurons typically exhibited a pronounced augmentation of their EPSC responses (Fig.11 H). (Jockusch et al., 2007). Interestingly, only a few CAKI cells showed moderate augmentation of their EPSC responses $2 \mathrm{~s}$ after HFS and the majority of cells remained either silent (26\%) or released only minute 
levels of transmitter that likely reflected the fusion of one to very few SVs (Fig.11 H). Similarly, application of PDBu failed to potentiate EPSCs in CAKI cells, while controls showed an augmentation of release by a factor of $\sim 1.6$ (Fig.11 H).

Altogether, the results of this section indicate that the profound residual priming activity observed in CAPS DKO neurons after HFS or PDBU potentiation is virtually abolished by introduction of the H567K mutation in Munc13-1. The C1 domain of Munc13s may thus play a crucial role in partially compensating for the loss of CAPS proteins.

A
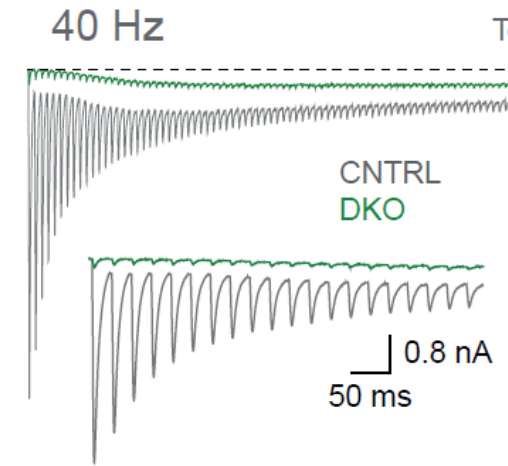

C $40 \mathrm{~Hz}$ $\sqrt{\sqrt{\text { CATRL }}}$

E

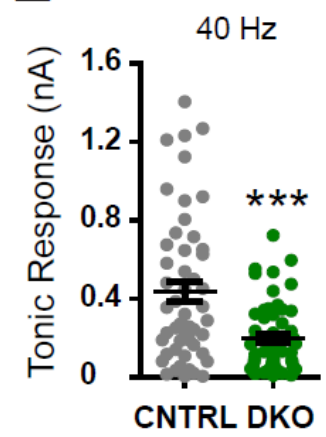

$\mathrm{F}$

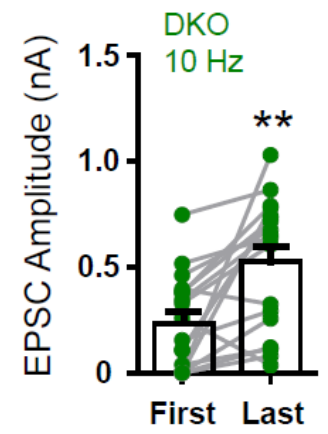

Tonic Response

$$
\begin{array}{l|l} 
& 0.8 \mathrm{nA} \\
0.3 \mathrm{~s} &
\end{array}
$$

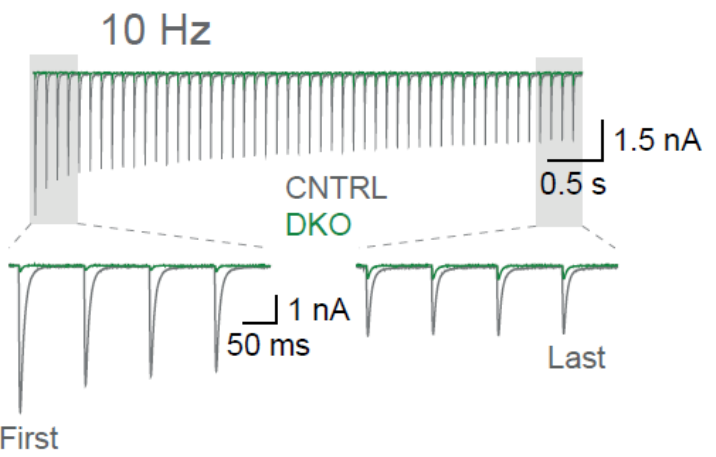

$\mathrm{D}$

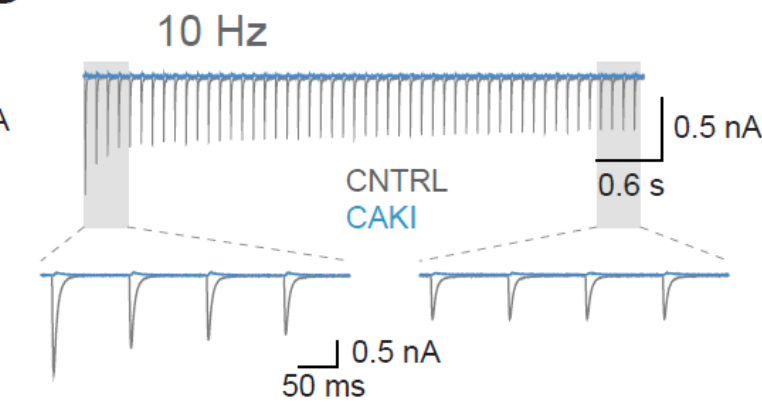

Figure 11: Transmitter release can neither be rescued by PDBu, nor by trains of APs in CAKI cells in contrast to CAPS DKO neurons. (A) Averaged EPSC responses during a train of $100 \mathrm{APs}$ at $40 \mathrm{~Hz}$. The inset depicts a magnification of the first 20 EPSCS and tonic responses used for quantification are highlighted in gray in top traces ( $n=50$ and 54 cells from $N=6$ cultures, Mann-Whitney test, $\left.{ }^{* * *} P=0.0007\right)$. (B) Averaged EPSC responses during a train of 50 APs at $10 \mathrm{~Hz}$. The insets depicts a magnification of the first-and last 4 EPSCS that are highlighted in gray in top traces ( $n=17$ and 18 cells from $N=2$ cultures, Mann-Whitney test, $\left.{ }^{* *} P=0.0075\right)$. (C) and (D) Averaged recordings in 
correspondence to (A) and (B) ( $n=64$ and 70 cells from $N=3$ cultures for (C); Mann-Whitney test, ${ }^{* * *} P<0.0001$, $n=24$ cells each from $N=2$ cultures for (D)). (E) Scatterplot of averaged tonic responses highlighted in gray in (A). (F) Dose-response plot of first- and last EPSC responses of recordings shown in (B). (G) Scatterplot of averaged tonic responses highlighted in gray in (C). (H) EPSC amplitude before and after $40 \mathrm{~Hz} A P$ trains (left) and in absence or presence of $3 \mu \mathrm{M} P D B u$ (right) ((left) $n=64$ of 74 and 19 of 74 CAKI cells from $N=3$ cultures did not show APevoked EPSCs before or after $40 \mathrm{~Hz}$ stimulus application, respectively, and were excluded from analysis, KruskalWallis test, Dunn's multiple comparison test, ${ }^{* * *} P<0.0001$, (right) $N=20$ of 21 and 16 of 21 CAKI cells from $N=2$ cultures did not exhibit AP-evoked responses in absence or presence of PdBu, respectively, and were not included in analysis, two-tailed t-test, $\left.{ }^{*} P=0.0376\right)$. (CNTRL $=$ same is for experiments in Fig. $1+2$, Bars in plots represent mean and SEM).

\subsubsection{Electrophysiological dissection of the structure function relationship of CAPSs during SV priming}

Despite the identification of CAPS proteins as essential priming- and docking factors for the release of transmitter, only little information is available on their structure-function relationship (Imig et al., 2014; Jockusch et al., 2007; Walent et al., 1992). Most of this information is based on data obtained from studies monitoring release from DCVs in permeabilized, or intact PC12 cells, as well as in cultured neuropeptidergic cells from the nematode C.elegans (Grishanin et al., 2002; Grishanin et al., 2004; Kabachinski et al., 2016; Khodthong et al., 2011; Lin et al., 2010; Petrie et al., 2016; Zhou et al., 2007). In recent years, a model of the function of CAPSs during priming has evolved, according to which CAPSs tether secretory vesicles via a C-terminal DCV-binding site and a PH domain that interacts with $\mathrm{PIP}_{2}$ at presynaptic release sites (Grishanin et al., 2002; James et al., 2008; Lin et al., 2010). Following the tethering step, CAPSs are thought to utilize their MUN domain to assemble trans-SNARE complexes required for subsequent fusion (Khodthong et al., 2011; Lin et al., 2010; Parsaud et al., 2013). In order to functionally assess the validity of this model in the context of SV priming, I carried out a structure function study of CAPSs in cultured glutamatergic hippocampal neurons. In collaboration with the research group of Jens Rettig, I focused my analysis particularly on three naturally occurring splice variants that either contained all known protein domains (CAPS2b), contained a deletion within the PH domain (CAPS2C), or lacked the entire C-terminal half of the protein that comprises the MUN domain and the DCV-binding site (CAPS2e) (Fig.12) (Sadakata et al., 2007a). The research group of Jens Rettig studied release from DCVs in cultured adrenal chromaffin cells, while I performed experiments on autaptic neurons, as indicated above. Some results of this study were published in a joint publication (Nguyen*, Nestvogel* et al., 2014). 


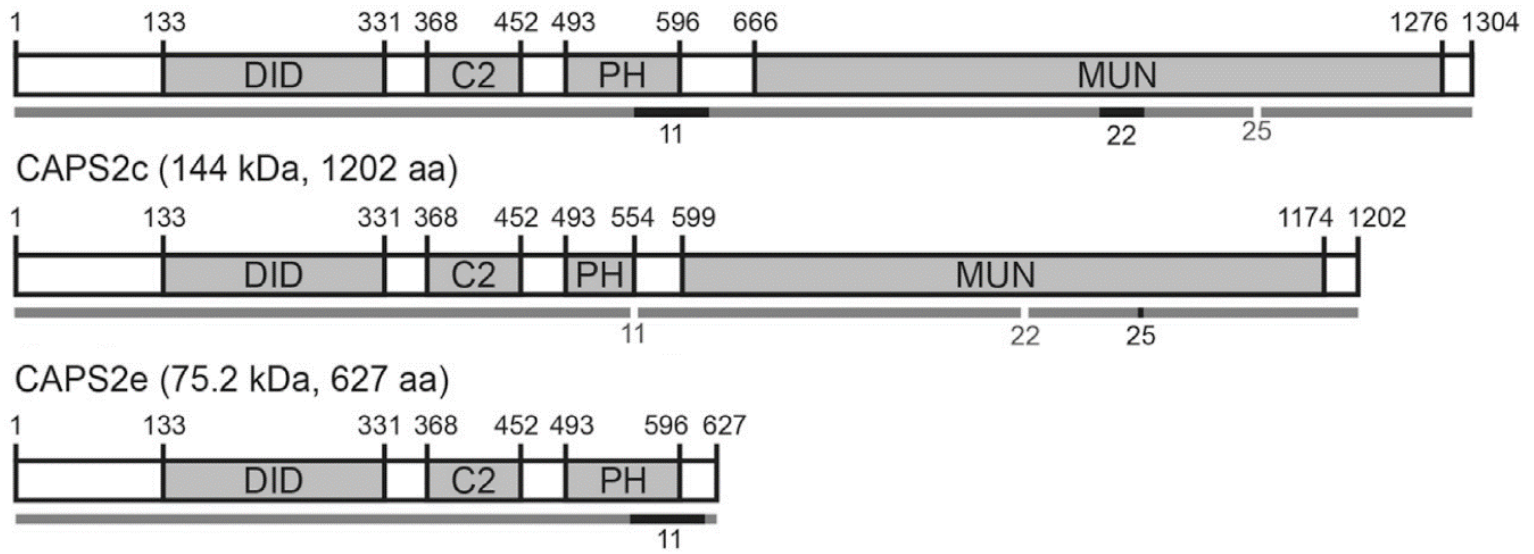

Figure 12: Domain structure of CAPS2 splice variants. Domain boundaries are illustrated by shaded regions and black values indicate amino acids at the respective boundaries. Splicing of exons that results in different protein variants is depicted below domain structures (modified after Nguyen et al., 2014).

\subsubsection{The SNARE-binding MUN domain and the DCV binding site of CAPSs are not essential for SV priming}

First, I studied the functional significance of the C-terminal DCV binding site of CAPSs, as well as of the SNARE-interacting MUN domain during SV priming. For this, I overexpressed the CAPS2e splice variant in cultured CAPS DKO neurons using Semliki Forest viruses and studied synaptic transmission using CAPS2 $b$ expressing cells as controls. Based on previous findings of an essential role of the C-terminal half in peptidergic release by PC12 cells and neurons from C.elegans, I expected to observe a pronounced rescue in CAPS2b expressing DKO cells, while transmitter release would remain perturbed in presence of CAPS2e (Grishanin et al., 2002; Khodthong et al., 2011; Lin et al., 2010; Parsaud et al., 2013). Surprisingly, however, AP-evoked responses were similarly rescued by overexpression of either one of the two splice variants, resulting in significantly higher EPSC amplitudes when compared to CAPSdeficient cells (Fig.13 A, B). Application of sucrose pulses for the measurement of RRP charges further revealed that both splice variants increased the number of primed vesicles, although there was a clear tendency that these charges were twice as large in CAPS2b- than in CAPS2e expressing cells (Fig.13 C). As a direct consequence of this, expression of CAPS2e yielded higher values for the vesicular release probability, indicating that the C-terminal half of CAPSs rather fulfills a modulatory function in transmitter release than being essential for generating a basic pool of SVs that is required for AP-evoked responses at rest (Fig.13 D). 


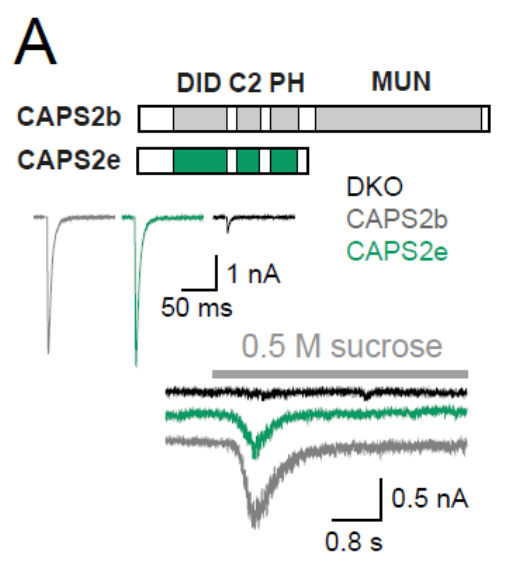

B

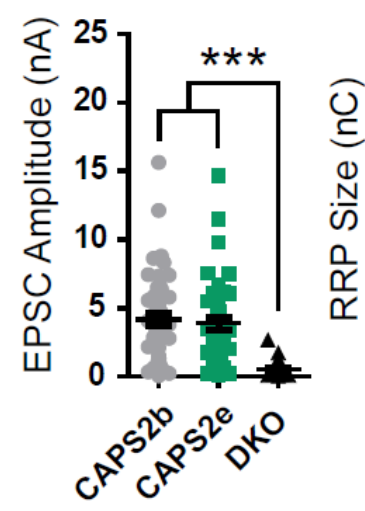

C

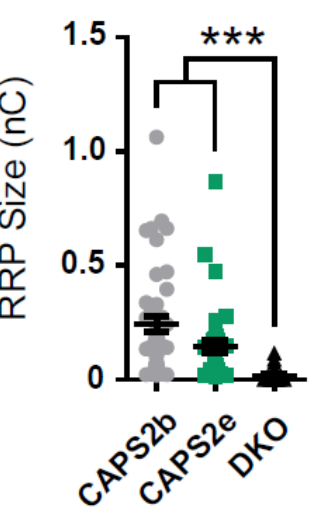

$\mathrm{D}$

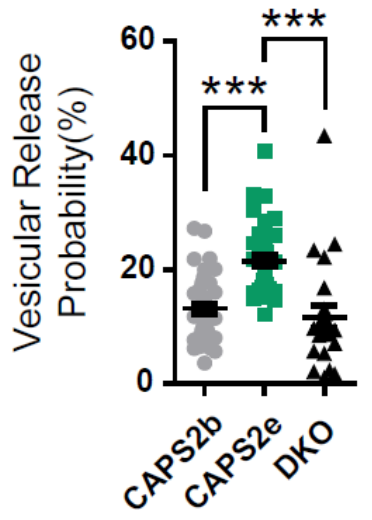

Figure 13: The C-terminal half of CAPS2 is not essential for basic synaptic transmission. (A) Domain structure of CAPS2 splice variants used for Semliki Forest virus-mediated overexpression in cultured CAPS DKO neurons and example recordings of AP- and sucrose triggered EPSCS. (B) Mean amplitude of EPSCS evoked by single APS ( $n=49$, 43 and 45 cells, of which 12 DKO cells showed no AP-triggered release and were excluded from analysis, KruskalWallis test, Dunn's Multiple Comparison Test, ${ }^{* * * P}<0.0001$ ). (C) RRP size determined by applying $0.5 \mathrm{M}$ sucrose solution and measuring the resultant charge $(n=43,36$ and 32 cells, Kruskal-Wallis test, Dunn's Multiple Comparison Test, $\left.{ }^{* * * P}<0.001\right)$. (D) Mean vesicular release probability calculated by dividing the charge of APevoked EPSCs by the charge of sucrose evoked responses ( $n=43,36$ and 23 cells). (Bars in plots depict mean and SEM. $N=8$ cultures, some of the data was published in Nguyen et al., 2014).

Following the characterization of AP- and sucrose evoked release at rest, I studied the functional significance of the C-terminal half of CAPSS in SV priming at elevated levels of presynaptic activity. I speculated that the increased vesicular release probability in CAPS2e expressing neurons would become manifest as an enhancement of short-term synaptic depression (Rhee et al., 2002).

Strikingly, application of AP trains at 10 and $40 \mathrm{~Hz}$, respectively, led to a significantly stronger depression of EPSC amplitudes in CAPSe- than in CAPS2b expressing cells (Fig.14 A-D). This depression was most obvious in the early phases of AP trains, particularly during the first two pulses, as indicated by strong reductions in the PPRs. Specifically, the PRR for two consecutive EPSCs with a time interval of $100 \mathrm{~ms}$ was reduced in presence of CAPS2e by $39 \%$ when compared to CAPS2b expressing cells and by even $46 \%$ at stimulations with a time interval of $25 \mathrm{~ms}$ (Fig.14 C). However, no statistically significant differences could be detected at these stimulation frequencies for EPSC amplitudes at late stages of AP trains, as reflected by the steady state levels of phasic EPSC responses (Fig.14 D). In addition, EPSCS recovered to a similar extend $2 \mathrm{~s}$ after $40 \mathrm{~Hz}$ AP trains in both groups (CAPS2b, $1.217 \pm 0.256$ and CAPS2e, $0.963 \pm 0.077$ ), while uninfected DKO cells showed prominent augmentation of their EPSC amplitudes by a factor of $\sim 7.2$, which is similar to the previously reported value of $\sim 9$ (Fig.14 E) (Jockusch et al., 2007). This particular observation corroborates earlier findings that activity-dependent priming, which has been shown to become boosted by increases in residual $\mathrm{Ca}^{2+}$ during HFS, is not impaired in absence of CAPSs (Hosoi et al., 2007; Jockusch et al., 2007). Indeed, the activity dependent replenishment rate of releasable vesicles was found to be similar between the three groups, as determined by plotting the 
cumulative charge of EPSC responses as a function of time and calculating the slope of linear fittings that were applied to the curves at steady-state stages of release (Fig.15 A,B) (Neher, 2015).

A<smiles>[R16][14CH3]</smiles>

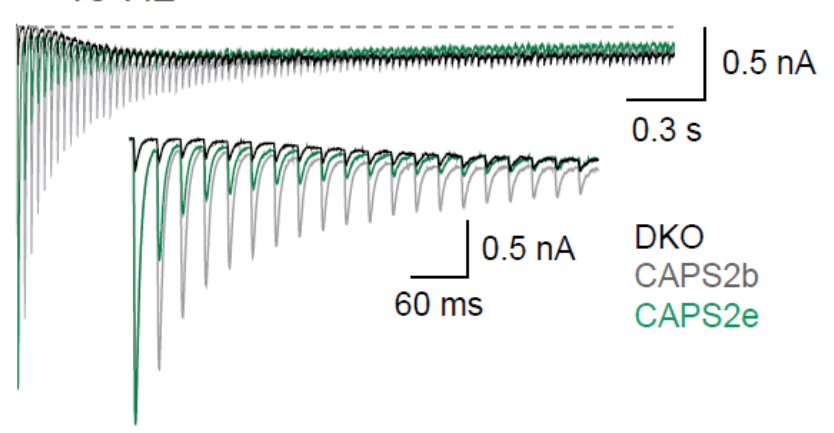

C

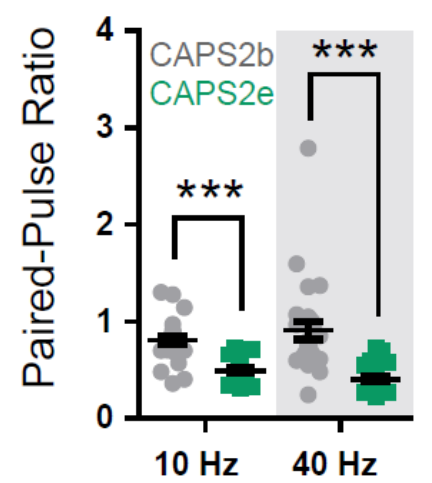

D

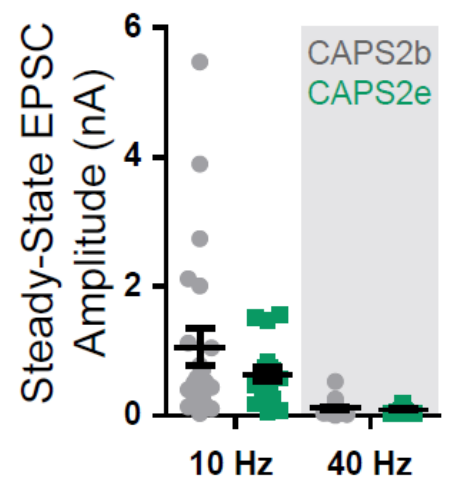

B

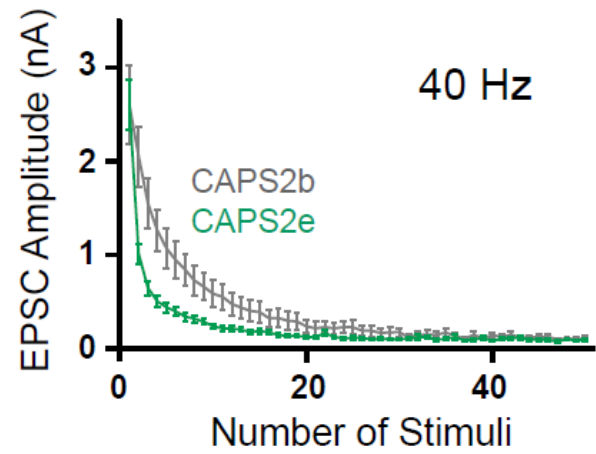

$\mathrm{E}$

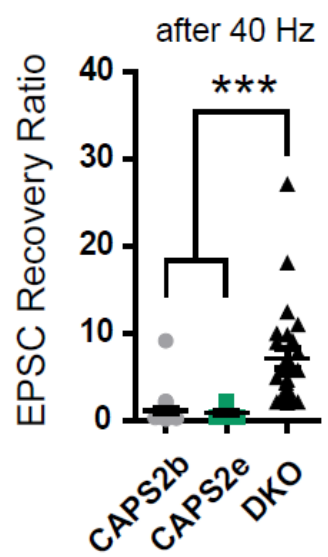

Figure 14: Short-term synaptic transmission is altered upon deletion of the C-terminal half of CAPS2. (A) Averaged EPSC responses evoked by a train of $100 \mathrm{APs}$ at $40 \mathrm{~Hz}$ in cultured DKO cells expressing indicated CAPS2 splice variants ( $n=24,26$ and 22 cells) and (B) mean amplitude of the phasic release component during stimulation. (C) Paired-pulse ratio calculated by dividing the second EPSC by the first EPSC response during AP trains at the indicated frequencies ( $n=23$ and 18 cells for $10 \mathrm{~Hz}$, two-tailed t-test, *** $p<0.001,26$ and 22 cells for $40 \mathrm{~Hz}$, Mann Whitney test, $\left.{ }^{* *} P<0.001\right)$. (D) Averaged steady-state EPSC responses during trains of APs at indicated frequencies $(n=23$ and 18 cells for $10 \mathrm{~Hz}, 26$ and 22 cells for $40 \mathrm{~Hz}$, averaged steady-state was measured between the $40^{\text {th }}$ and $50^{\text {th }}$ stimulus). (E) Recovery of EPSCS after HFS given as the ratio between the EPSC response $2 \mathrm{~s}$ after 100 AP pulses at $40 \mathrm{~Hz}$ and the first EPSC response during the AP train ( $n=34,31$ and 25 cells, Kruskal-Wallis test, Dunn's Multiple Comparison Test, ${ }^{* * *} P<0.001$ ). (Bars in plots depict mean and SEM. $N=4-7$ cultures, some of the data was published in Nguyen et al., 2014).

Next, I tested whether deletion of the C-terminal half of CAPSs would affect the refilling of RRP vesicles at rest. Since CAPSs proteins seem to be dispensable for activity-dependent refilling, as suggested by the results above and by the study of Jockusch et al. (2007), I hypothesized that the tendency of a reduced RRP size in CAPS2e expressing cells may reflect a specific priming impairment at low residual $\mathrm{Ca}^{2+}$ levels. To test this hypothesis, I applied two consecutive hyperosmotic sucrose pulses separated by a time interval of $2 \mathrm{~s}$ and measured the recovery of RRP charges. Since sucrose pulses are thought to 
not lead to significant increases in the residual $\mathrm{Ca}^{2+}$ concentration, in contrast to depletion of RRP vesicles by HFS, the recovery of such responses should reflect the replenishment, or repriming, of releasable vesicles at rest. Strikingly, the mean recovery ratio of the RRP charge was found to be reduced in CAPS2e expressing DKO cells by about $61 \%$ when compared to CAPS2b expressing neurons, indicating a partial impairment of the priming process at low intraterminal $\mathrm{Ca}^{2+}$ levels (Fig.15 C, D).
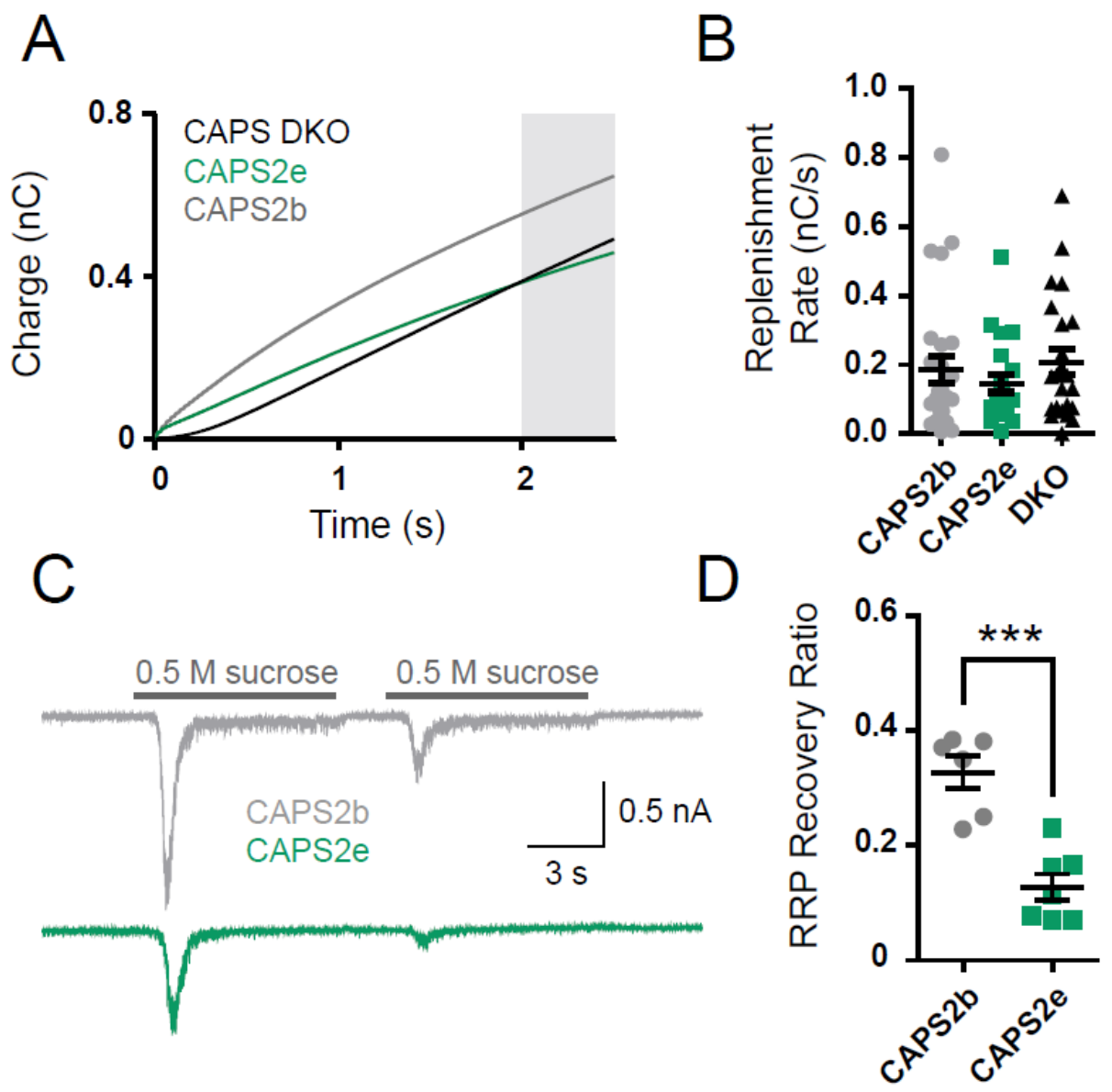

Figure 15: SV priming is partially impaired in CAPS2e expressing neurons at basic levels of presynaptic activity. (A) Averaged cumulative charge of EPSCS responses shown in Fig.4. The shaded box indicates the time interval in which linear fittings were applied to curves for the quantification of activity-dependent RRP replenishment rates that are illustrated in (B) ( $n=24,26$ and 22 cells). (C) Representative traces for double-sucrose pulses that were applied to study the recovery of RRP vesicles at resting conditions. The recovery ratios shown in (D) were calculated by dividing the charge of the $2^{\text {nd }}$ sucrose-evoked EPSC by the charge triggered by the $1^{\text {st }}$ sucrose stimulus ( $n=6$ and 7 cells, respectively). (Bars in plots depict mean and SEM. $N=1-7$ cultures).

Overall, the data presented here suggest that the C-terminal half of CAPSs is not essential to support SV priming for the release of transmitter in response to single APs at rest. However, once a second AP pulse invades the nerve terminal after a short period, transmitter release is impaired in presence of CAPS2e as reflected by a pronounced decrease in the PPR. Based on the findings of a somewhat smaller RRP charge, which yields higher values in the calculation of vesicular release probabilities for CAPS2e- than 
for CAPS2b cells, it is likely that the C-terminal half of CAPSs is involved in establishing an additional number of RRP vesicles that do not significantly contribute to the release of transmitter during single APs (see discussion).

\subsubsection{The central PH domain of CAPSs is essential for basic transmitter release}

CAPS1 has been shown to bind acidic phospholipids via its central PH domain, with a particularly strong stereoselectivity for $\mathrm{PIP}_{2}$ (Grishanin et al., 2002). PIP 2 in turn, was reported to regulate important aspects of transmitter release, including RRP size, STP and frequency of spontaneous fusion events (Di Paolo et al., 2004; Gong et al., 2005; Milosevic et al., 2005). Molecularly, PIP 2 was shown to be the dominant inner-leaflet lipid ( $82 \%$ surface coverage) in syntaxin-1 membrane clusters at release sites that have been described to serve as docking sites for secretory vesicles (Honigmann et al., 2013; van den Bogaart et al., 2011). Besides CAPSs, other proteins of the release machinery have been reported to bind PIP2, such as synaptotagmin-1 and Munc13s (Bai et al., 2004; Park et al., 2015; Schiavo et al., 1996; Shin et al., 2010). Even though synaptotagmin-1 has mechanistically been identified to constitute the molecular factor that mediates $\mathrm{PIP}_{2}$ dependent recruitment of vesicles to syntaxin clusters, it seems unlikely that this interaction is responsible for the function of $\mathrm{PIP}_{2}$ in regulating the RRP size, based on findings that deletion of synaptotagmin-1 in mice does not lead to changes in the number of primed SVS in cultured autaptic neurons and that docking of SVs is not substantially impaired in synaptotagmin-1 KO organotypic slices (Imig et al., 2014; Liu et al., 2009). Similarly, manipulation of the PIP2-binding C2B domain of the priming and docking factor Munc13 was only shown to lead to prominent changes in STP, while the RRP size remained unchanged in cultured neurons (Shin et al., 2010). Thus, interactions between $\mathrm{PIP}_{2}$ with synaptotagmin-1 and Munc13s may modulate certain aspects of release, but the molecular mechanism by which PIP 2 exerts its known function in regulating RRP size remains unknown at the present time. Interestingly, previous results from reconstitution assays using permeabilized PC12 cells as model systems for regulated secretion have shown that acidic phospholipid and $\mathrm{PIP}_{2}$ interactions are essentially required for the activity of CAPSs in transmitter release (Grishanin et al., 2002). Thus, one likely scenario by which $\mathrm{PIP}_{2}$ may regulate the size of the RRP is by regulating the activity of CAPS proteins. To test this hypothesis, I expressed the CAPS2c splice variant that contains a damaged PH domain resulting from a short deletion and tested its rescue capacity in CAPS DKO neurons in comparison to full-length CAPS2b (Fig. 16). The deletion present in the PH domain of CAPS2c led to the removal of a structurally conserved alpha helix and thus likely to a significant perturbation in the capacity of CAPSs to bind PIP 2 and other acidic phospholipids (Fig. 16 A,B). Despite this severe structural perturbation, however, expression of the CAPS2c splice variant in BHK cells yielded protein bands in 
western blot experiments at the expected molecular weight indicating that the protein expressed properly and was not severely misfolded (western blot analysis carried out by members of the Rettig lab, see Nguyen et al., 2014). Since the protein sequence of the PH domain of CAPSs is highly conserved across species for all known isoforms, I expected that findings from this study on CAPS2 could be extrapolated to CAPS1 function (Grishanin et al., 2002).

Interestingly, a basic characterization of CAPS2c expressing DKO neurons revealed a deficit in synaptic transmission, which resembled that of uninfected controls (Fig.16 C-F). In detail, AP- and sucrose triggered EPSCS were not significantly different between CAPS2c- and DKO cells, while CAPS2b expression led to pronounced increases in evoked responses (Fig.16 C, D). As a direct consequence of this, no further differences in the vesicular release probability between these groups could be detected (Fig.16 E). Interestingly, AP-evoked EPSCS were strongly augmented $2 \mathrm{~s}$ after application of HFS in CAPS2c cells, similarly to CAPS DKO neurons indicating that this particular splice variant does not display a dominant negative effect on residual $\mathrm{Ca}^{2+}$-dependent priming activity (Fig. 16 F). Thus, previous reports on an essential role of the PH domain of CAPSs in transmitter release by DCV could also be validated in this study for glutamatergic synaptic transmission.

A

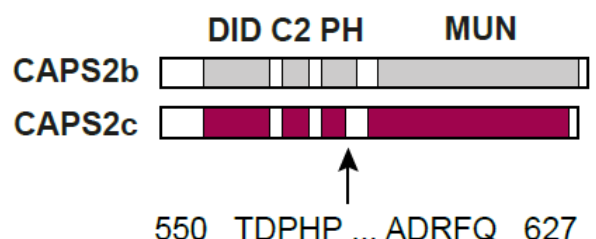

C

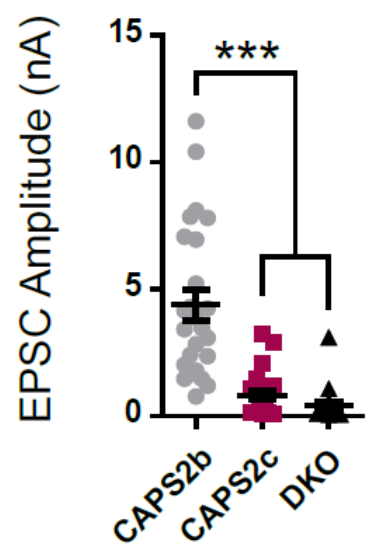

B

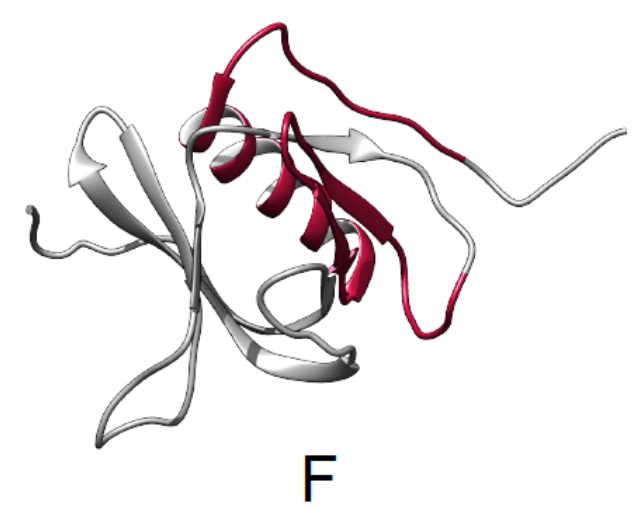

$E$

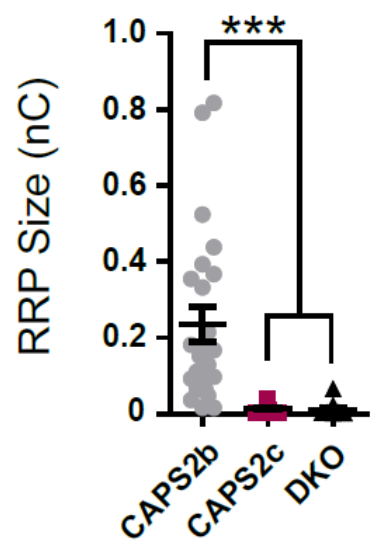

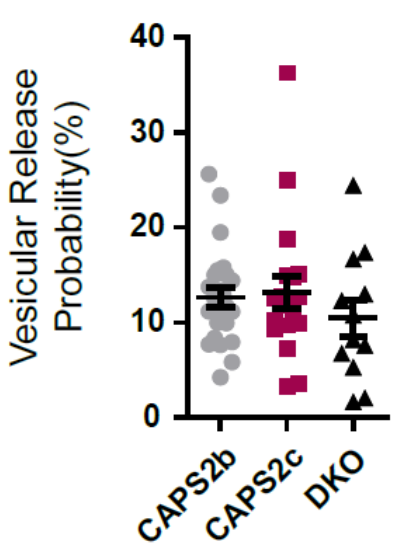

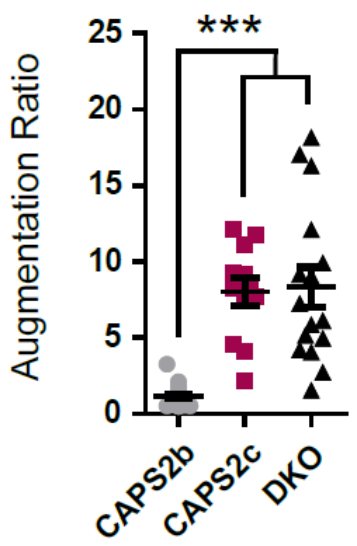

Figure 16: CAPS dependent priming requires a functional PH domain. (A) Domain structure of CAPS2 splice variants used for Semliki Forest virus-mediated overexpression in cultured CAPS DKO neurons. The arrow and amino acid sequence indicate the site of deletion within the PH domain of CAPS2c. (B) Three dimensional solution NMR 
structure of the human CADPS1 PH domain (PDB: 1wi1, chain A \#0.3). The homologous sequence that is deleted in CAPS2C is highlighted in red. Molecular graphics and analyses were performed with UCSF Chimera package (Pettersen et al. 2004). (C) Mean EPSC amplitude evoked by single APs ( $n=25$ cells each, 2 CAPS2c expressing-, and 4 DKO cells showed no AP-triggered EPSCs and were excluded from analysis, Kruskal-Wallis test, Dunn's Multiple Comparison Test, $\left.{ }^{* * *} P<0.001\right)$. (D) RRP size measured by application of $0.5 \mathrm{M}$ sucrose solution $(n=24,22$ and 16 cells, Kruskal-Wallis test, Dunn's Multiple Comparison Test, ***P<0.001). (E) Vesicular release probability $(n=24$, 19 and 12 cells). (H) Augmentation of EPSCs after HFS given as the ratio between the EPSC response $2 \mathrm{~s}$ after 100 AP pulses at $40 \mathrm{~Hz}$ and the first EPSC response during the AP train $(n=22,12$ and 16 cells, Kruskal-Wallis test, Dunn's Multiple Comparison Test, ${ }^{* * *} P<0.001$ ). (Bars in plots depict mean and SEM. $N=4$ cultures, Data published in Nguyen et al., 2014).

Alternatively, the partial deletion within the PH domain of CAPSs may have additionally led to structural changes in other regions of the protein that in turn may not have become apparent during western blot analysis and thus could have possibly confounded the study. In order to address this, I studied the effect of a mutation that selectively impairs lipid binding by the $\mathrm{PH}$ domain. Key residues that are essential for $\mathrm{PIP}_{2}$ interactions are located within the loop between the $\beta 3$ and $\beta 4$ sheets of the domain and are highly conserved throughout the animal kingdom in CAPS1 and CAPS2 (Fig.17 A,B) (Grishanin et al., 2002; James et al., 2008). In a previous study it was shown that charge reversal of these key residues largely abolishes the interaction between CAPSs and $\mathrm{PIP}_{2}$ and furthermore impairs CAPS-dependent acceleration of artificial liposome fusion when $\mathrm{PIP}_{2}$ containing liposomes are used as donors and phosphatidyl choline/phosphatydylserine containing liposomes are used as acceptors (James et al., 2008). By using site-directed mutagenesis, I introduced corresponding mutations into the PH domain of the CAPS2e splice variant that had been shown in previous experiments of this study to be sufficient to establish a basic RRP pool for AP-evoked release in CAPS DKO cells (see above).

Similarly to overexpression experiments in CAPS DKO cells using CAPS2c, expression of the mutated CAPS2e splice variant using semliki forest viruses ("RKK") did neither increase AP, nor sucrose evoked EPSCs (Fig.17 C-E). Accordingly, the specific interaction between CAPSs and PIP 2 is essentially required to sustain synaptic transmission by regulating the RRP size. 
A

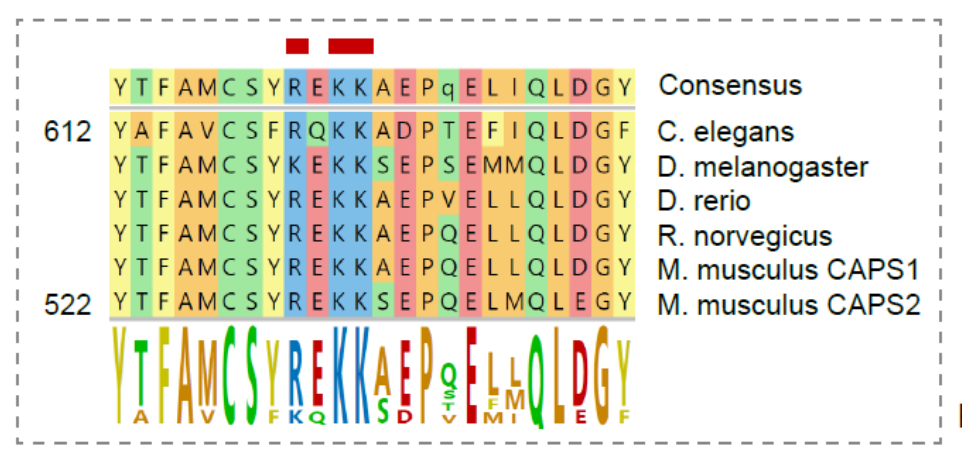

C

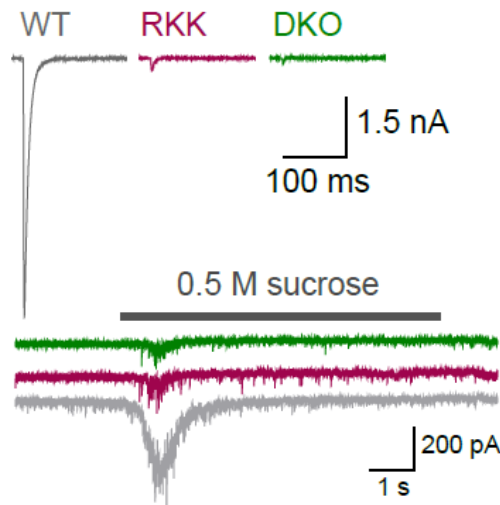

B

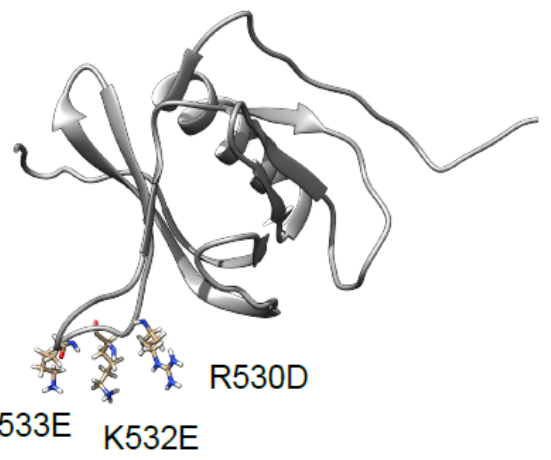

E

Figure 17: CAPS dependent priming requires a PIP 2 binding site within the PH Domain. (A) Positively charged residues (highlighted in blue, red bar) within the PH domain of CAPS are highly conserved across species and were previously reported to be essential for PIP 2 binding by CAPSs (Grishanin et al., 2002; Grishanin et al., 2004, James et al., 2008). Sequence alignment (Clustal Omega) and analyses were performed using MegAlign Pro, DNASTAR Incorp. (B) Three dimensional solution NMR structure of the human CADPS1 PH domain (PDB: 1 wi1, chain A \#0.3). Positively charged residues are positioned in loop between $\beta 3$ - and $\beta 4$ sheets and were mutated to negatively charged residues for charge reversal in correspondence to earlier studies (Grishanin et al., 2002; Grishanin et al., 2004, James et al., 2008). Molecular graphics and analyses were performed with UCSF Chimera package (Pettersen et al. 2004). (C) Example recordings of AP- and sucrose triggered EPSCS. (D) Mean EPSC amplitude evoked by single APs in CAPS2e WT- and CAPS2 $e_{R K K}$ expressing cells ( $n=11,10$ and 10 cells, 1 CAPS2 $e_{R K K}$ expressing-, and 4 DKO cells showed no AP-triggered EPSCS and were excluded from analysis, Kruskal-Wallis test, Dunn's Multiple Comparison Test, ${ }^{* * P}<$ $\left.0.01,{ }^{* *} P<0.001\right)$. (E) RRP size determined by measuring sucrose-evoked EPSCS ( $n=11,10$ and 6 cells, One-way ANOVA, Bonferroni's Multiple Comparison test, ${ }^{* *} p<0.01,{ }^{* * *} p<0.001$ ). (Bars in plots depict mean and SEM. N = 1 culture). 


\subsubsection{Deletion of a conserved protein region within the DI- domain of CAPSs does not lead to changes in transmitter release}

The findings described in previous sections of this study suggest that the principal activity of CAPSs in establishing a basic number of RRP vesicles for AP-triggered release is encoded within the N-terminal half of the protein. Despite the identification of the $\mathrm{PIP}_{2}$ binding $\mathrm{PH}$ domain as a crucial determinant of this activity, it remains largely unknown to what extend other $\mathrm{N}$-terminal regions of the protein contribute to the molecular reactions by which CAPS2e positively modulates the RRP size.

Two other known domains within CAPS2e include the C2 domain, as well as a protein region that was identified in a yeast-two hybrid screen to bind the p150glued subunit of dynactin (DI) and is referred to in the literature as the DI-domain (Sadakata et al., 2007a; Sadakata et al., 2007b). The interaction between dynactin and CAPS2 has been validated by coimmunoprecipitation (Sadakata et al., 2007b). However, due to the significant differences in the cell biological functions of both proteins the physiological relevance of this interaction remains controversial. Specifically, dynactin regulates the trafficking of cell organelles by recruiting the motor protein dynein to microtubules (Urnavicius et al., 2015). Knock-down of its homologue in C.elegans, DNC-1, leads to axonal accumulation of autophagosomes, accompanied by a severe degeneration of motor neurons. On the other hand, a direct role of dynactin in the release of transmitter has not been described in the literature, in contrast to CAPS proteins. Yet, despite this seeming discrepancy, previous studies reported a reduction in the axonal localization of CAPS2 and lower levels of BDNF release by cultured hippocampal neurons and PC12 cells, respectively, upon partial deletion of the DI-domain, which was shown to occur by aberrant splicing in a subset of ASD patients (Sadakata et al., 2012; Sadakata et al., 2007b). Moreover, C.elegans worms that lack a strikingly conserved amino acid stretch within the DI-domain exhibit motor phenotypes resembling those of unc31 null allele worms (Speese et al., 2007). Thus, irrespective of whether the binding of $p 150^{\text {glued }}$ is of physiological relevance, the DI-domain of CAPS2 may play an important role in sustaining transmitter release.

In order to test a direct involvement of the DI-domain in the regulation of the RRP size by CAPS2e, I generated a mutant cDNA construct, in which a short stretch of amino acids with a high degree of conservation across isoforms and species was deleted by site-directed mutagenesis (Fig.18 A). The stretch I chose for my overexpression experiments in autaptic neurons consisted of five amino acids ("WIAKY") and was located within the protein region previously reported to be essential for proper motor function in C.elegans and to be required for BDNF release by cultured cells (Sadakata et al., 2012; Sadakata et al., 2007b; Speese et al., 2007). I chose to introduce a short deletion within this region 
rather than deleting it entirely in order to minimize possible side effects on the structure and folding of other domains in CAPSs, which in turn could have possibly confounded my analysis.

In correspondence to rescue experiments described above, I used Semliki Forest viruses to introduce WT-CAPS2e and mutant CAPS2 $\mathrm{e}^{\text {WIAKY }}$ in cultured autaptic neurons derived from CAPS DKO mice. Application of single APs or sucrose yielded no significant differences in the amplitude of evoked EPSCS between CAPS2 $\mathrm{e}^{\text {WIAKY}}$ - and WT-CAPS2e expressing cells, thus resulting in similar values for the vesicular release probability, as well (Fig.18 C-E). Moreover, the amplitude and frequency of mEPSCs was similarly increased by expression of either one of the two cDNA constructs indicating that deletion of the highly conserved stretch of amino acids within the DI-domain does not compromise the activity of CAPS2e in supplying the neuronal synapse with RRP vesicles for basic synaptic transmission (Fig.18 F, G).

Next, I tested whether expression of CAPS2 $\mathrm{e}^{\text {WIAKY }}$ would lead to altered forms of STP. Interestingly, expression of either CAPS2 $\mathrm{e}^{\text {WIAKY }}$ or CAPS2e WT equally prevented the strong augmentation of transmitter release $2 \mathrm{~s}$ after HFS stimulation in CAPS DKO neurons and no further differences between the two groups could be observed when AP-evoked responses were measured during trains of APs at frequencies of 10 and $40 \mathrm{~Hz}$ (Fig.18 H-J). Collectively, these results argue against a direct role of the conserved stretch of amino acids within the DI-domain in regulating the activity of CAPS2e at basic-and elevated levels of presynaptic activity. However, these results do not exclude the possibility of an indirect involvement of the $\mathrm{DI}$-domain in synaptic transmission by modulating the axonal trafficking of CAPS2, as reported in previous studies (Sadakata et al., 2012; Sadakata et al., 2007b). Accordingly, further experiments in the future are required to address this issue and to additionally dissect the contribution of the C2 domain of CAPSs during synaptic transmission (see discussion). 
A

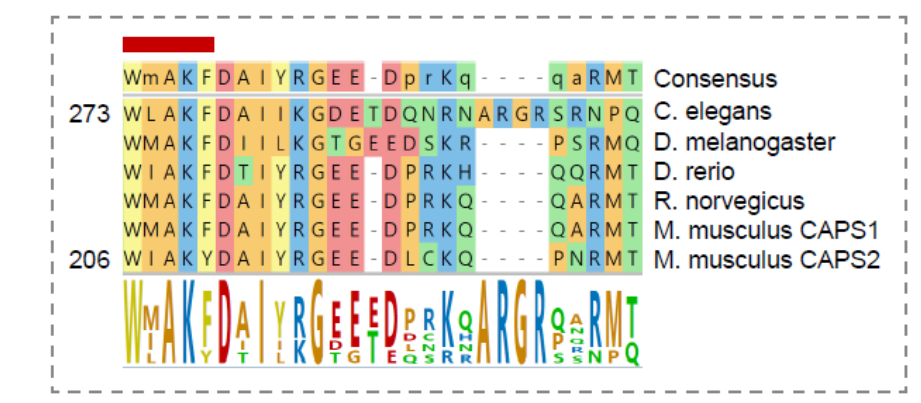

C

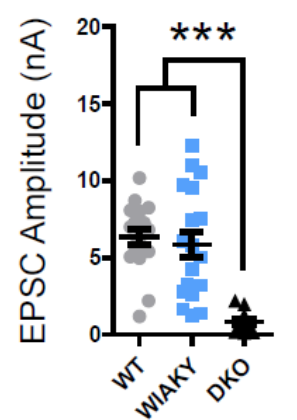

$\mathrm{H}$

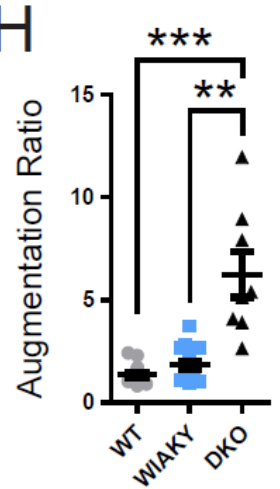

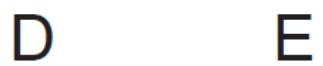

E

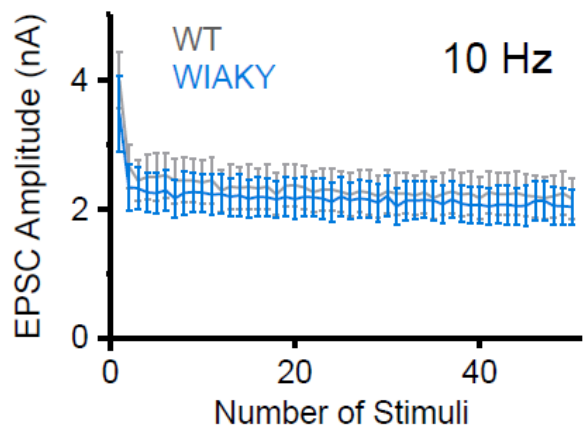

B

i)

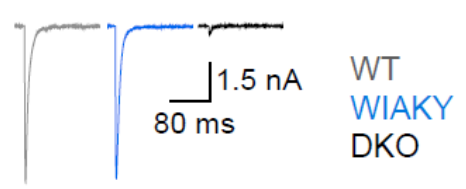

ii)

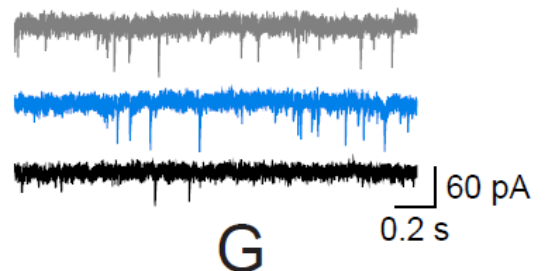

$\mathrm{F}$
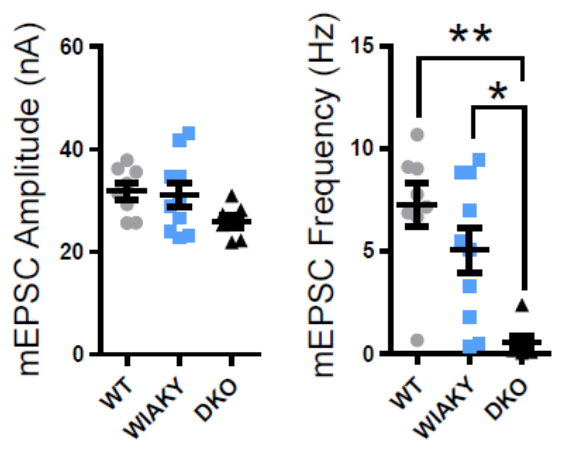

$J$

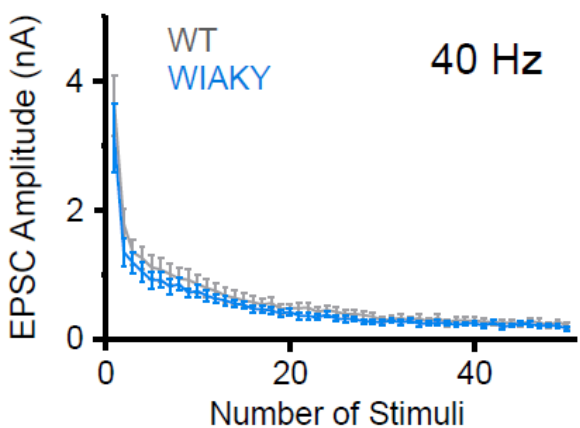

Figure 18: A highly conserved stretch of amino acids within the DI-domain of CAPS is not essential for SV priming. (A) Sequence alignment of a highly conserved region within the DI-domain of CAPS that was previously reported to be essential for locomotion in c.elegans (Speese et al., 2007). A short stretch within this region (red bar) with the highest degree of conservation across species was mutated in this study for functional assessment ("WIAKY"). Sequence alignment (Clustal Omega) and analyses were performed using MegAlign Pro, DNASTAR Incorp. (B) Representative traces of AP-evoked EPSCS (i) and spontaneously occurring mEPSCS (ii). (C) Mean amplitude of APevoked EPSCs ( $n=18,18$ and 16 cells, of which 4 DKO cells exhibited no responses, One-way ANOVA, Bonferroni's Multiple Comparison test, ${ }^{* * *} P<0.001$ ). (D) RRP size determined by applying $0.5 \mathrm{M}$ sucrose solution and measuring the evoked charge ( $n=17,18$ and 7 cells, Kruskal-Wallis test, Dunn's Multiple Comparison Test, ${ }^{*} P<0.05,{ }^{*} P<$ 0.01). (E) Mean vesicular release probability calculated by dividing the charge of AP-evoked EPSCs by the charge of sucrose evoked responses ( $n=17,18$ and 5 cells). (F) and (G) Mean amplitude and frequency of spontaneously occurring mEPSCS ( $n=8,10$ and 7 cells, Kruskal-Wallis test, Dunn's Multiple Comparison Test, ${ }^{*} P<0.05,{ }^{*} P<$ 0.01). (H) Augmentation of EPSCS after HFS determined by calculating the ratio between the EPSC response $2 \mathrm{~s}$ after $100 \mathrm{AP}$ pulses at $40 \mathrm{~Hz}$ and the first EPSC response during the AP train $(n=14,16$ and 8 cells, Kruskal-Wallis test, Dunn's Multiple Comparison Test, ${ }^{* * P}<0.01,{ }^{* * * P}<0.001$ ). (I) and (J) Mean EPSC responses during a train of APs at frequencies of 10 and $40 \mathrm{~Hz}(n=15$ and 16 cells (for $10 \mathrm{~Hz}$ ); $n=14$ and 15 cells (for $40 \mathrm{~Hz}$ ). (Bars in plots depict mean and SEM. $N=3$ cultures). 


\subsubsection{Overexpression of CAPS2e is sufficient to sustain AP- evoked release in CAKI cells}

The findings described above indicate that protein lipid interactions are crucially involved in generating RRP vesicles. In detail, the PIP ${ }_{2}$-binding domain of CAPSs is required to sustain a basic RRP at rest for transmitter release in response to single APs, while the DAG-binding C1 domain regulates $\mathrm{Ca}^{2+}$ dependent priming for release during and after high levels of presynaptic activity (Fig. 11, 16 and 17). By abolishing these protein lipid interactions via deletion of CAPSs and introduction of the H567K mutation into Munc13-1, transmitter release is largely diminished as demonstrated by experiments on cultured CAKI cells. One possible explanation of these findings may be given by postulating the existence of a minimal priming machinery for AP-evoked release consisting of a SNARE-interacting MUN domain of Munc13s, based on previous reports, and further, of a lipid-interacting domain supplied by the PH domain of CAPSs, or the C1 domain of Munc13s (Basu et al., 2005). To test this hypothesis, I prepared cultured cells derived from the hippocampus of CAKI mice and overexpressed the MUN-domain lacking CAPS2e splice variant, whose catalytic activity involves $\mathrm{PIP}_{2}$ binding. Based on my hypothesis, I expected that overexpression of CAPS2e would suffice to establish a basic RRP for AP-evoked release. As a control, I used full-length CAPS2b expressing CAKI cells that I speculated to resemble the previously published features of Munc13-1 ${ }^{\text {H567K }} /$ Munc13-2 double mutant neurons (Rhee et al., 2002).

A basic analysis of synaptic transmission revealed a pronounced rescue of EPSCs triggered by single APs in both CAPS2b- and CAPS2e expressing CAKI cells when compared to uninfected controls (Fig.19 A, B). Even though there was a slight tendency that EPSCs were smaller in CAPS2e containing cells, a statistical analysis revealed no significant differences between the two groups. Furthermore, EPSC responses evoked by application of hyperosmotic sucrose solution were increased upon expression of either one of two splice variants, with a clear tendency of a larger RRP size in CAPS2b- than in CAPS2e cells (Fig.19 C). These results support findings from previous experiments of this study, where both CAPS2e and CAPS $2 b$ were shown to sustain release triggered by single APs equally well, while CAPS $2 b$ may recruit an additional number of vesicles into the RRP for release during prolonged stimulation. In addition, these results suggest that the activity of CAPS2e in promoting a basic pool of primed vesicles does not depend on an intact $\mathrm{C} 1$ domain in Munc13-1. 
A

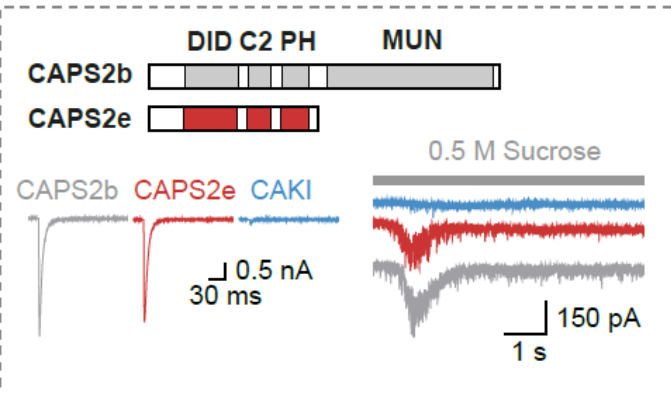

$\mathrm{D}$

$10 \mathrm{~Hz}$

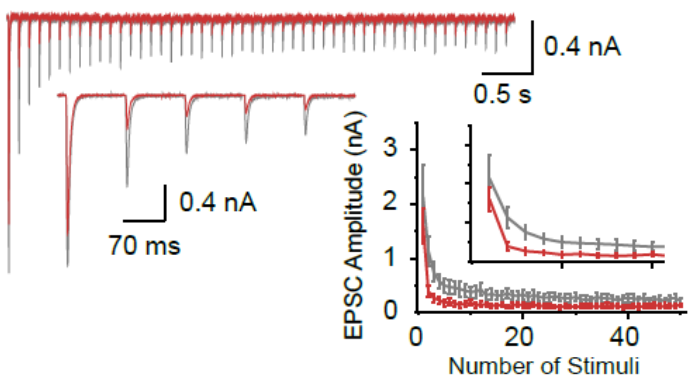

F

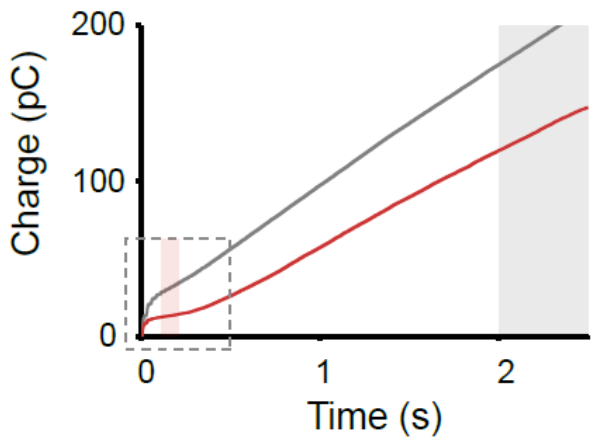

$\mathrm{B}$

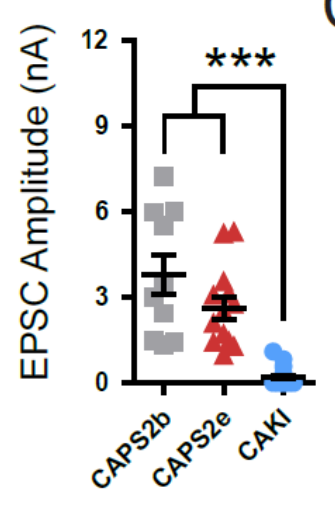

E
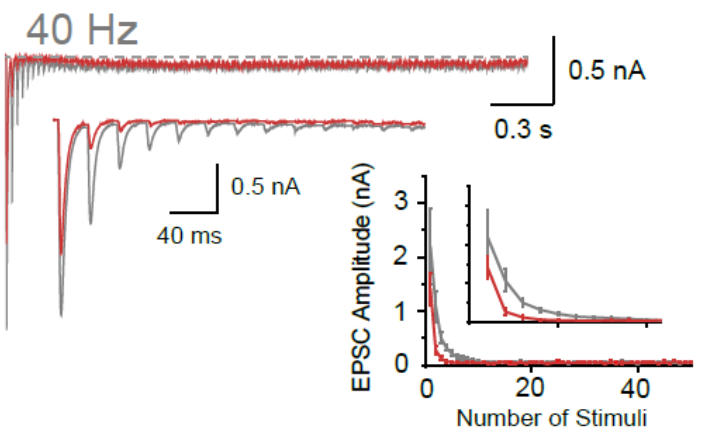

G

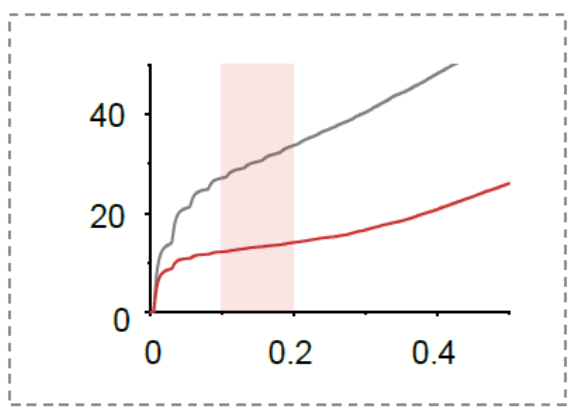

K

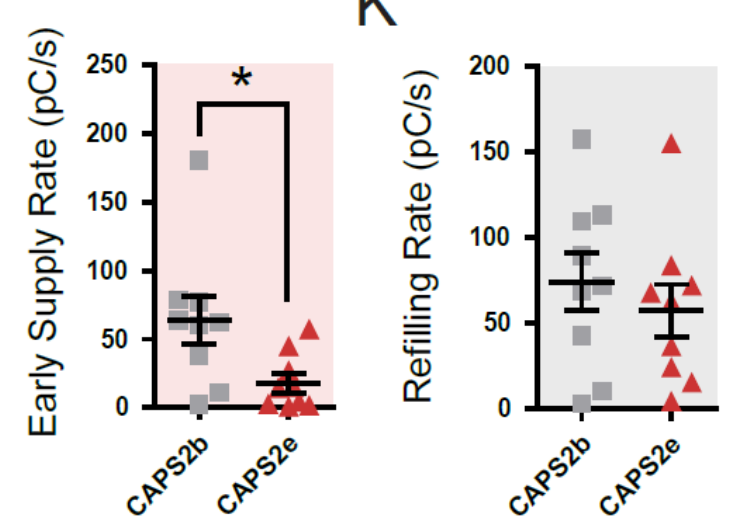

Figure 19: Overexpression of MUN domain lacking CAPS2e is sufficient to rescue basic transmitter release in CAKI neurons. (A) Domain structure of CAPS2 splice variants used for rescue experiments in cultured cells and representative traces for AP- and sucrose evoked EPSCS. (B) and (C) EPSC amplitude and RRP charge measured by applying single APs or $0.5 \mathrm{M}$ sucrose solution, respectively ((B) $n=10,13$ and 19 cells, of which 5 CAKI cells exhibited no AP-triggered release, Kruskal-Wallis test and Dunn's Multiple Comparison test, ${ }^{* * * P}<0.0001$, (C) $N=8,9$ and 15 cells, of which 10 CAKI cells did not show sucrose evoked EPSCS, Kruskal-Wallis test and Dunn's Multiple 
Comparison test, ${ }^{* * *} P=0.0008$ ). (D) and $(E)$ Averaged recordings of EPSCS during AP trains at indicated frequencies: The Insets depict magnifications of trace, as well as graphs, in which the tonic release component was removed (unpaired t-test, $\left.{ }^{* * *} P<0.001\right)$. (F) Cumulative charge of recordings in (E) and (G) magnification of dashed box shown in (F). (H) Paired-pulse ratio for recordings shown in (D) and (E) ( $n=9$ cells, unpaired t-test, $* * P=0.0064$ and $* * P=0.0065)$. (I) Recovery of EPSC amplitudes given as the ratio of EPSCs before and $2 \mathrm{~s}$ after the train of 100 $A P s$ at $40 \mathrm{~Hz}$ (unpaired t-test, $P=0.2685)$. (J) and $(K)$ Slopes of linear fittings in red-and gray shaded regions of curves shown in (F) (Mann-Whitney test, ${ }^{*} P=0.0142$ and unpaired $t$-test, $P=0.4708$ ). (Bars in scatterplots represent mean and SEM, $N=2$ cultures).

I next applied trains of APs to study evoked release during elevated levels of presynaptic activity. As indicated above, CAKI cells fail to exhibit tonic or phasic release during HFS in contrast to CAPS DKO neurons. This in turn may suggest that the $\mathrm{C} 1$ domain of Munc13s is crucially involved in the regulation of $\mathrm{Ca}^{2+}$-dependent priming of SVs. Accordingly, I expected that while AP-evoked release during early phases of AP trains would be increased by expression of CAPS2b- and CAPS2e, respectively, evoked release at late phases of AP trains would largely remain perturbed and would resemble that of previously published Munc13-1 ${ }^{\text {H567K }} /$ Munc13-2 double mutant cells.

Strikingly, EPSC responses depressed very rapidly upon expression of either one of the two CAPS2 splice variants in CAKI cells in response to prolonged stimulation at frequencies of 10 and $40 \mathrm{~Hz}$ (Fig.19 D, E). This depression of amplitudes was even more pronounced in CAPS2e- than in CAPS2b expressing cells as expected from the initial observation of differences in RRP size between the two groups. The cumulative plot of EPSC charges during $40 \mathrm{~Hz}$ AP trains further revealed that RRP vesicles were almost entirely depleted in CAPS2e cells after 3-5 stimuli in contrast to CAPS2b cells suggesting that this population of SVs exhibited high release probabilities and a large proportion of these fused with the plasma membrane at the first stimulus (Fig.19 F, G).

An analysis of late stages of evoked release during prolonged stimulation revealed a small component of tonic release that was present in both rescue conditions (Fig.19 E). Interestingly, a recent study that applied $\mathrm{Ca}^{2+}$ uncaging to the study of synaptic transmission in cultured neurons reported that tonic release stems from the ongoing fusion of newly primed vesicles rather than from pool of vesicles that is primed before HFS (Burgalossi et al., 2010). Accordingly, this may indicate that priming at high activity levels is not completely abolished in CAPS2 expressing CAKI cells in contrast to uninfected controls. This priming activity, in turn, may have reflected the baseline priming rate that operates at rest, or it may have been mediated by a $\mathrm{Ca}^{2+}$ dependent priming activity that does not require the $\mathrm{C} 1$ domain of Munc13s. Since CAPS2e reprimes vesicles more slowly than CAPS2b at rest, a complete shutdown of the $\mathrm{Ca}^{2+}$ dependent priming component should have yielded differences in the magnitude of the tonic release between the two conditions. Such a difference, however, could not be observed. Furthermore, the recovery of EPSC responses after HFS, as well as the priming rate during prolonged stimulation 
should be smaller in CAPS2e than in CAPS2b cells under the assumption of a complete shutdown of $\mathrm{Ca}^{2+}$ dependent priming at high activity.

To address this issue in greater detail, I studied the recovery of EPSC responses after HFS stimulation. Interestingly, EPSC responses recovered to an equal extent $2 \mathrm{~s}$ after HFS in both CAPS2 $\mathrm{b}$ and CAP2e cells, but tended to be smaller when compared to overexpression experiments in CAPS DKO cells, supporting published results indicating that the $\mathrm{C} 1$ domain of Munc13s modulates the recovery of EPSCS after HFS (Fig.19 I)(Rhee et al., 2002). Furthermore, the RRP refilling rate at late stages of release were found to not be different between the two conditions, as revealed by plotting the cumulative charge of EPSC responses as a function of time and calculating the slope of linear fits that were applied to the curves at steady-state stages of release (Fig.19 J,K). These results suggest that $\mathrm{Ca}^{2+}$ dependent refilling of RRP vesicles is similar between CAPS2b-and CAPS2e expressing CAKI cells and it is not entirely diminished compared to uninfected neurons. It therefore is unlikely that the baseline priming activity at rest fully accounts for the tonic release, as well as for the recovery of EPSCs during and after HFS. Rather, it is likely that some unknown $\mathrm{Ca}^{2+}$ dependent priming process that does not require the $\mathrm{C} 1$ domain of Munc13s, while depending on the presence of CAPSs may regulate the tonic release, as well as the recovery of EPSCs during and after HFS in CAPS2 expressing CAKI cells.

Although these results collectively support my hypothesis of a minimal priming machinery (see above), alternative interpretations of the data could not be ruled out completely and are discussed in a different section of this thesis (see discussion).

\subsection{The relevance of CADPS1 for early-onset bipolar disorder}

A number of mutations were recently identified in the human CADPS1 gene in a subset of early-onset BPD patients (Sitbon et al., unpublished results; Fig.20). This finding raised the question, as to whether any of these mutations may lead to an impairment of CAPS1 function and may thus be causally involved in the etiology of the disease.

To address this, I carried out rescue experiments in cultured CAPS1,2 DKO neurons, in which I expressed mutant CADPS1 cDNA constructs using lenti viruses. As described in an earlier section of this thesis, lenti viral expression of cDNA typically yields protein levels that are comparable to those of endogenously expressed proteins and is therefore ideally suited to prevent strong overexpression, which in turn may mask deficiencies in protein function arising from reduced stability, transport or mislocalization. I had already analyzed the effect of some of these mutations on synaptic transmission before I started my PhD project (Master's thesis; N205K, L482I, R959L and N1017I). However, many of the results were preliminary and not conclusive. Furthermore, the protocol for producing lentiviruses did not yield 
reproducible amounts of virus particles at that time and may thus have led to discrepancies during rescue experiments. Accordingly, I reinvestigated the effect of these mutations on synaptic transmission during my PhD project and extended the study to the analysis of the remaining mutations that I had not studied before (Exon2 deletion, R195L, S399L and double L482I/N1017I). All of the data in this study were solely collected during my PhD studies and none of the data from the master studies are included in this thesis.

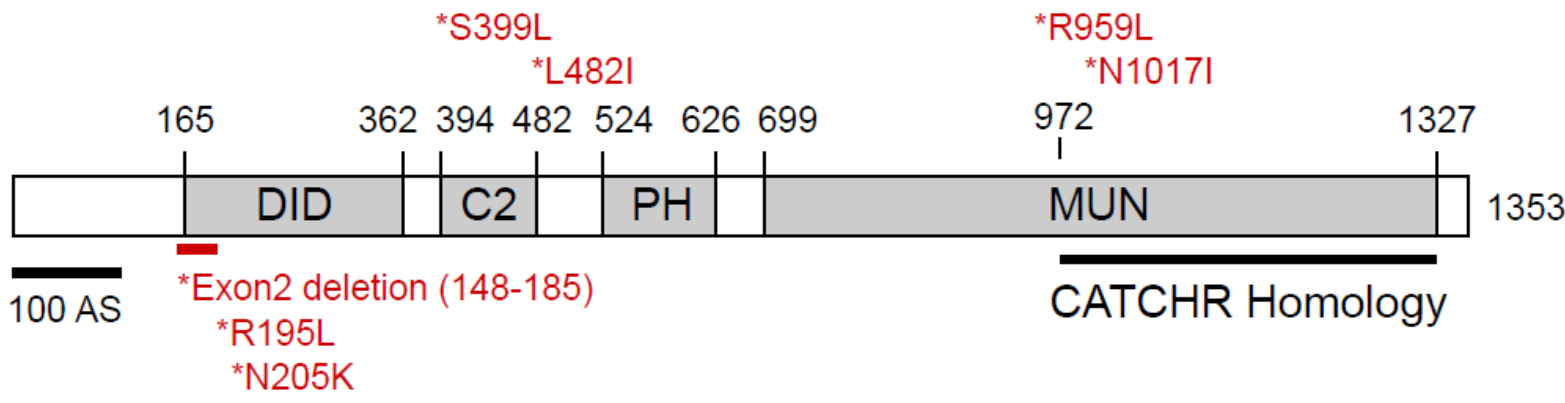

Figure 20: Domain structure of human CADPS1 splice variant 1. Black values indicate domain boundaries (Sadakata et al., 2007, Nguyen et al., 2014). Black bar on right side indicates region with homology to CATCHR-type proteins (Pei et al., 2009). Bipolar disorder associated mutations are highlighted in red.

In correspondence to previous experiments of this study (see sections above), I carried out a battery of electrophysiological protocols to study various aspects of synaptic transmission in cultured CAPS DKO neurons expressing either mutated or WT CADPS1. These protocols included induction of single EPSCS to study AP-evoked release at rest, stimulation of cells by hyperosmotic sucrose solution to measure the RRP size, monitoring the frequency and amplitude of mEPSCs while TTX was applied, and studying STP characteristics at stimulation frequencies of 2, 5, 10 and $40 \mathrm{~Hz}$.

A detailed analysis using these protocols did not reveal any significant differences between WT- and R195L-, N205K-, L482I-, R959L-, N1017I- or L482I/N1018I expressing neurons, respectively (see supplementary figures 2-7). Accordingly, these mutations are not discussed in the following section in greater detail. Strikingly, however, expression of either CADPS1 ${ }^{\text {S399L }}$ or CAPDS1 ${ }^{\text {dEX2 }}$ led to significant changes in STP characteristics when compared to CADPS1 ${ }^{\text {WT }}$-expressing neurons, and these mutations are thus described in more detail below. 


\subsubsection{The S399L mutation within the C2 domain of CADPS1 leads to less pronounced short-term depression}

The S399L mutation is located within the $\mathrm{N}$-terminal region of the C2 domain of CADPS1. It was identified in two BPD patients who shared the same parents (Sitbon et al., unpublished results). Interestingly, one of the parents was initially diagnosed with unipolar depression without carrying any of the indicated CAPDS1 mutations. Thus it is likely that the S399L mutation was passed on to the progeny by the other parent whose DNA was unfortunately not available for sequencing and who was reported to not exhibit any form of depression or other psychiatric disease.

Expression of CADPS1 ${ }^{\text {S399L }}$, or CADPS1 ${ }^{\mathrm{WT}}$ led to a similar increase in AP and sucrose evoked EPSCs in CAPS DKO cells with a tendency that EPSCS were slightly higher in presence of the mutation (Fig.21 A-D). However, no differences in the amplitude and frequency of mEPSCs were discovered between the two conditions, suggesting that basic synaptic transmission was not changed by the S399L mutation (Fig.21 $E, F)$

In contrast, application of AP trains at frequencies of 2,5 and $40 \mathrm{~Hz}$ revealed a statistically significant increase in the paired-pulse ratio, and EPSC amplitudes depressed less to prolonged stimulation during early phases of AP trains (Fig. $21 \mathrm{G}, \mathrm{H}$ ). However, these changes were not associated with a higher steadystate amplitude of phasic release during later stages of AP train (Fig.21 I).

Next, I analyzed the RRP replenishment rate during the $40 \mathrm{~Hz}$ AP train, as described above. Interestingly, CADPS1 ${ }^{\text {S399L }}$ expressing cells exhibited a significantly higher replenishment rate by a factor of 1.6 than CADPS $1^{\mathrm{WT}}$ controls, indicating that the $\mathrm{Ca}^{2+}$ dependent priming activity may have been enhanced by the S399L mutation (Fig.22 A,B). 


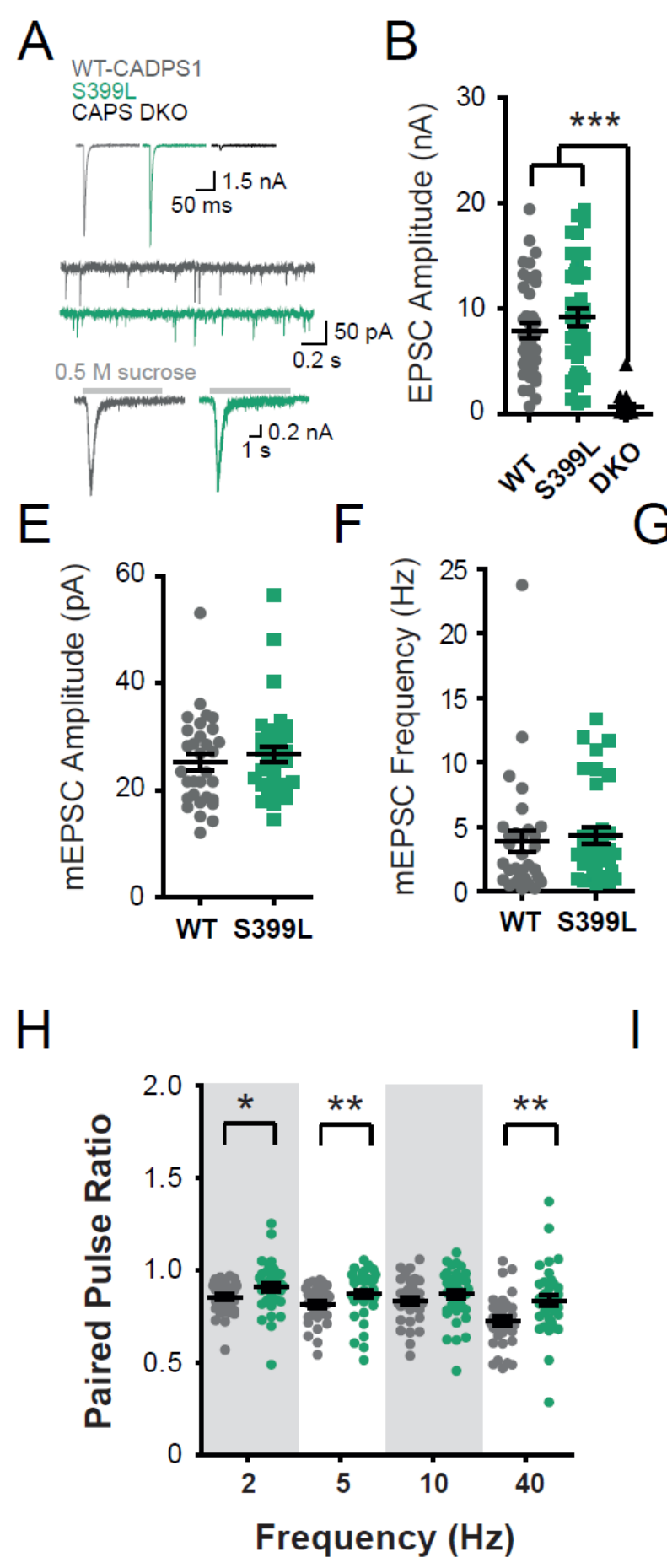

C D
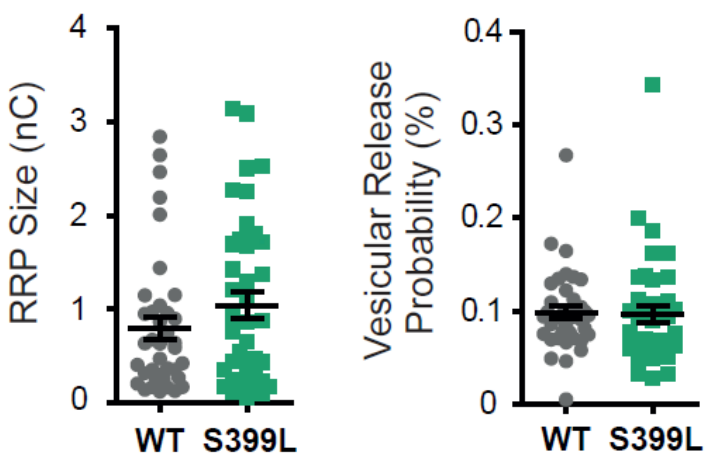

G
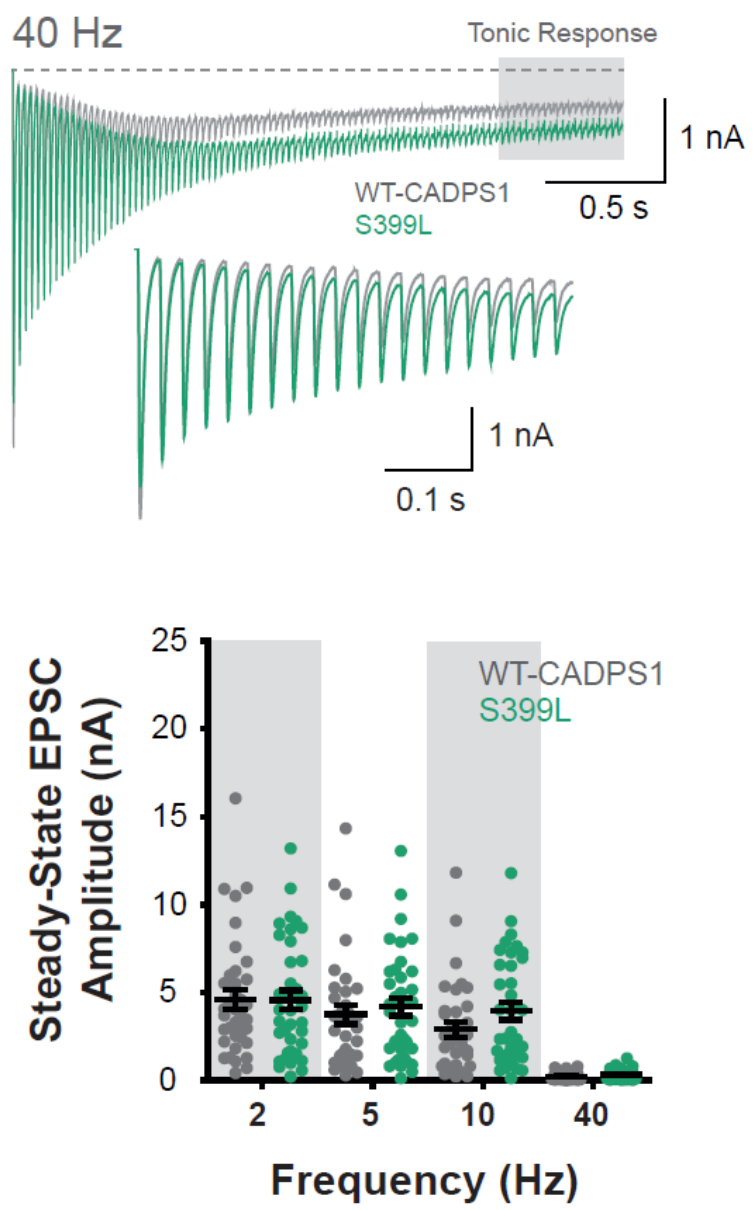

Figure 21: Cultured neurons expressing CADPS-15399L exhibit less short-term synaptic depression when compared to controls. (A) Sample traces of AP-evoked EPSCS (i) spontaneously occurring mEPSCS (ii) and sucrose-evoked EPSCS in cultured autaptic neurons. (B) Mean amplitude of AP-triggered EPSCs ( $n=40,41$ and 28 cells, of which 10 DKO cells did not show any AP-triggered responses, Kruskal-Wallis test, Dunn's Multiple Comparison Test, ${ }^{* * *} P<0.001$ ). (C) RRP charge measured in presence of $0.5 \mathrm{M}$ sucrose solution ( $n=38$ and 40 cells) (D) Vesicular release probability calculated by dividing the charge of AP-evoked EPSCS by the charge of sucrose evoked responses ( $n=38$ and 40 cells). (E) and (F) Mean amplitude and frequency of spontaneously occuring mEPSCs. ( $n=31$ and 34 cells). (G) Averaged EPSC responses during a $40 \mathrm{~Hz} A P$ train $(n=34$ and 36 cells) $(H)$ and (I) Paired-pulse ratio and steadystate EPSC responses during trains of APs at indicated frequencies ( $n=34-37$ cells, Mann Whitney test, ${ }^{*} P=0.0164$, ${ }^{*} P<0.01$ ). (Bars in plots depict mean and SEM. $N=6$ cultures). 
A

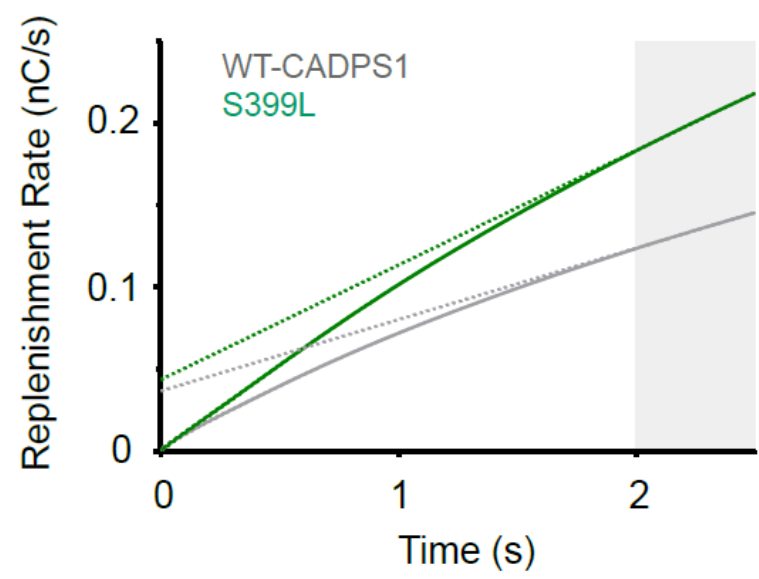

B

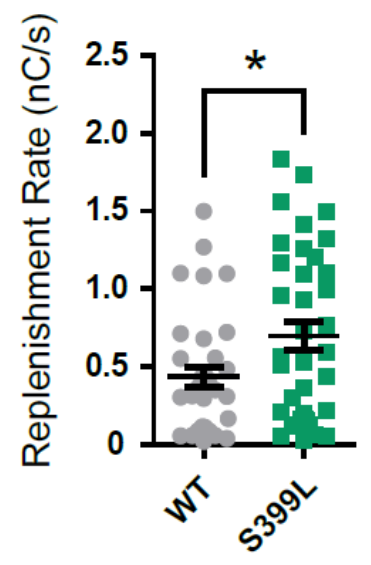

Figure 22: The S399L mutation in CADPS1 leads to a higher RRP replenishment during HFS. (A) Averaged cumulative charge of recordings shown in Fig. $15 \mathrm{G}$. The dashed lines represent the linear fittings that were applied to the gray shaded region of the curves. The slope of these fittings was interpreted as the RRP replenishment rate and corresponding values for each cell are shown in (B). (Bars in plots depict mean and SEM. N =6 cultures).

\subsubsection{Exon 2 deletion in CADPS1 leads to enhanced short-term synaptic depression}

The Exon 2 deletion ( $d E X 2$ ) in CADPS1 was identified in a female BPD patient, who notably was the only patient in the genetic study that had a parent diagnosed with the same disease (Sitbon et al., unpublished results). Unfortunately, however, the DNA of both parents was not available for the genetic study and conclusions on the genetic heritability of the gene deletion can thus not be drawn. The deletion itself leads to the expression of a protein that lacks 37 amino acids within the $\mathrm{N}$-terminal DIdomain. CAPS DKO cells, expressing either this mutated protein or CADPS1 ${ }^{\mathrm{WT}}$ showed similar increases in their AP- evoked responses when compared to uninfected control neurons (Fig.23 A,B). Interestingly, sucrose evoked charges in mutant CADPS1 ${ }^{\mathrm{dEX} 2}$ cells tended to be slightly decreased leading to an overall significant increase in the vesicular release probability compared to CADPS1 ${ }^{\text {WT }}$ neurons (Fig.23 C,D). This increase in vesicular release probability, in turn, was accompanied by a mild, yet significant enhancement of the mEPSC frequency, as well as by a pronounced decrease of PPRs at frequencies of 2,5,10 and $40 \mathrm{~Hz}$ (Fig.23 E-H). Further analysis of the steady state EPSC amplitudes, as well as of the replenishment rate (data not shown) revealed no differences for all of the frequencies tested, suggesting that the mutation only affects release during early phases of AP trains (Fig.23 I). 

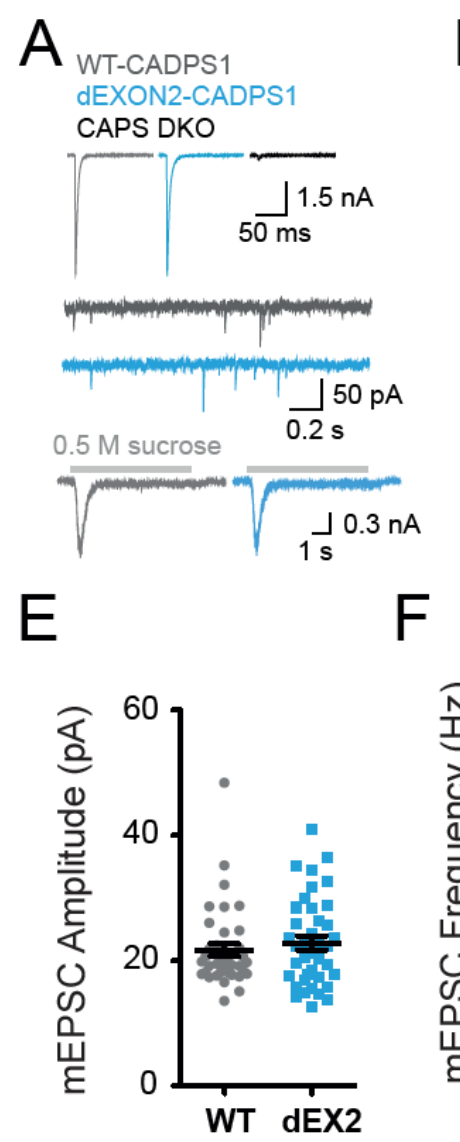

B

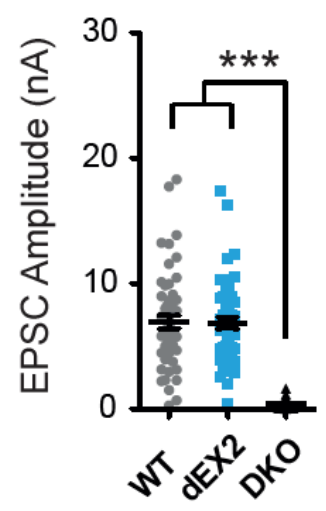

C

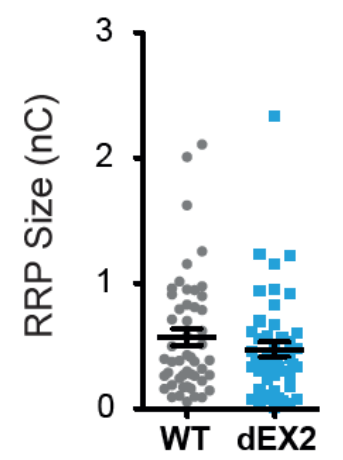

D

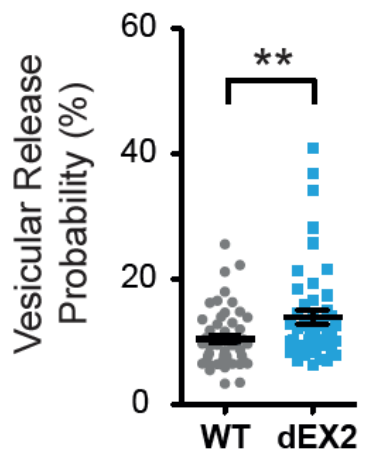

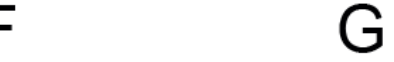

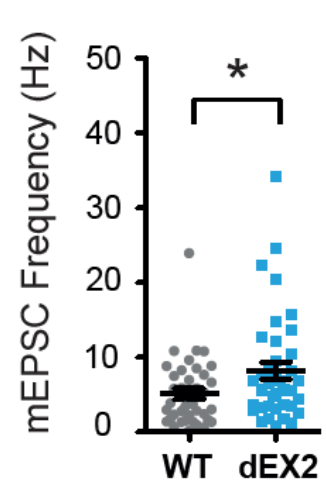

$40 \mathrm{~Hz}$

Tonic Response

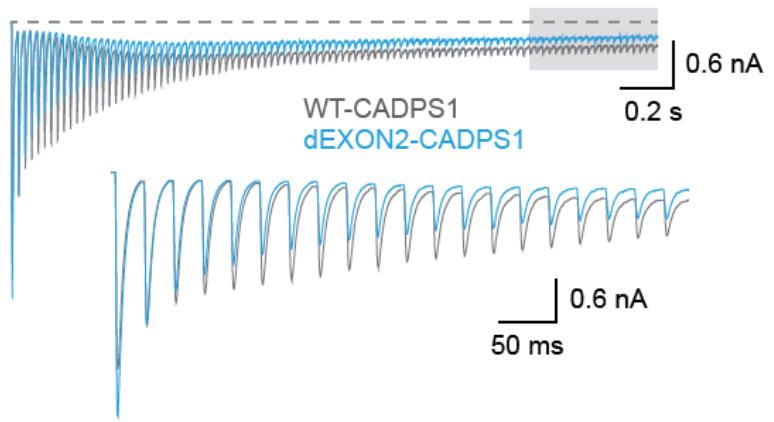

$\mathrm{H}$
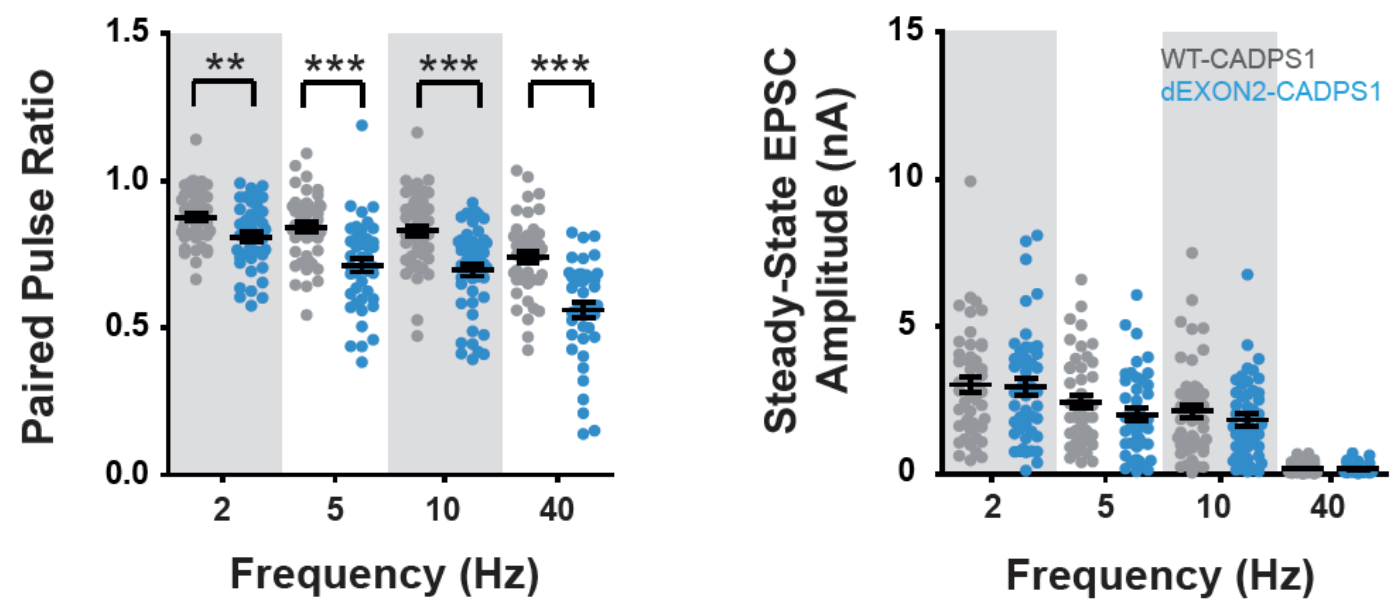

Figure 23: Cultured neurons expressing CADPS-1 ${ }^{d E x 2}$ exhibit more pronounced short-term synaptic depression when compared to controls. (A) Sample traces of AP-evoked EPSCS (i) spontaneously occurring mEPSCs (ii) and sucroseevoked EPSCs in cultured autaptic neurons. (B) Mean amplitude of AP-triggered EPSCs ( $n=53,50$ and 32 cells, of which 6 DKO cells did not show any AP-triggered responses, Kruskal-Wallis test, Dunn's Multiple Comparison Test, ${ }^{* * *} P<0.001$ ). (C) RRP charge measured in presence of $0.5 \mathrm{M}$ sucrose solution ( $n=50$ and 49 cells) (D) Vesicular release probability calculated by dividing the charge of AP-evoked EPSCS by the charge of sucrose evoked responses $(n=50$ and 49 cells). (E) and (F) Mean amplitude and frequency of spontaneously occuring mEPSCs. ( $n=39$ and 39 cells, Mann-Whitney test, $\left.{ }^{*} P=0.0317\right)$. (G) Averaged EPSC responses during a $40 \mathrm{~Hz}$ AP train ( $n=46$ and 41 cells) $(H)$ and (I) Paired-pulse ratio and steady-state EPSC responses during trains of APs at indicated frequencies $(n=41$ 49 cells, two-tailed t-test, ${ }^{* * P}<0.01,{ }^{* *} P<0.001$ ). (Bars in plots depict mean and SEM. $N=6$ cultures). 
In order to address the relevance of this mutation for neural circuit function and behavior in the future, I further collaborated with my colleagues Fritz Benseler and Ursula Fünfschilling to generate a mutant mouse strain carrying this mutation. The desired mutation was introduced genetically into mice using the novel CRISPR technique and it was detected by DNA sequencing in one out of 20 mice that were delivered by 7 female C57/BI6 J animals (Fig.24 and supplementary table 1) (Cong et al., 2013; Wang et al., 2013). We used the mouse carrying the desired mutation as a founder animal for the establishment of a new mouse line and the behavior of homozygous mutant animals is currently under investigation.

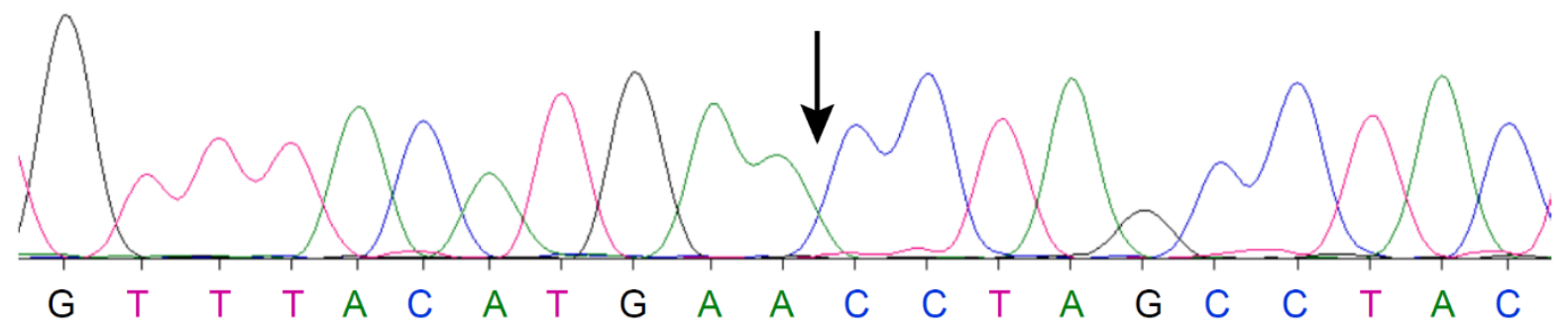

Figure 24: Sequence results of Exon2 deletion in the mouse CAPS1 gene. Sequencing results were obtained from tissue of mutant $d E X 2$ mice. The deletion of exon 2, as well as of putative splice acceptor sites was achieved using the novel CRISRP technique. The black arrow indicates the location of missing DNA bases.

\subsection{Development of an experimental paradigm for the study of SV priming factors in the context of neural circuit function}

Apart from hard wired anatomy, neurons rely on functional and dynamic connections in order to sustain information flow within neural circuits (Haider and McCormick, 2009). The functional connectivity is subject to moment-to-moment changes and can dynamically be adjusted to recent patterns of activity (Abbott and Regehr, 2004). For instance, synaptic responses in L4 cells depress in response to prolonged stimulation, which has been attributed to synaptic depression of the thalamic input (Chung et al., 2002; Kloc and Maffei, 2014; Lampl and Katz, 2017; Reinhold et al., 2015). The adaptation of evoked responses, in turn, is thought to modulate various response properties of $L 4$ neurons to sensory stimuli, such as contrast invariance and cross-orientation suppression, and may thus contribute to the neuronal representation of the external world (Carandini et al., 2002; Priebe and Ferster, 2012). The systematic dissection of the contribution of STP to sensory processing has been hampered by the difficulty to specifically manipulate it, while analyzing neural circuit function in living animals. In order to overcome this, I aimed at developing an experimental paradigm, in which the deletion of presynaptic proteins involved in the modulation of STP can specifically be introduced into selected axonal projections and sensory evoked responses can be measured in target regions. For this, I focused my efforts on the LGN to L4 axonal pathway of the mouse visual system. As a proof of principle, I selectively deleted CAPS-1 
and measured visually evoked subthreshold responses using the in-vivo blind patch clamp technique (Margrie et al., 2002).

\subsubsection{Targeting the deletion of presynaptic proteins to thalamic nuclei}

The conditional deletion of CAPS1 was achieved via the use of the Cre-loxP recombination system (Orban et al., 1992; Tsien, 2016; Tsien et al., 1996). For this, I generated a conditional KO (cKO) mouse strain with the help of ES cells that were purchased from EUCOMM (see methods). In a next step, I crossbred these mice with a SERTcre driver line to enable specific protein deletion in thalamic nuclei, including the LGN that is known to predominantly innervate layer 4 neurons in the visual cortex. Notably, cre expression in these mice was not limited to the thalamus but was also pronounced in other brain regions such as the raphe nucleus, cingulate cortex, CA3 region of the hippocampus and within a sparse population of cortical cells in accordance to what was published previously (Fig.25) (Narboux-Neme et al., 2008). Double-floxed mice on a cre positive genetic background were viable and did not exhibit any obvious phenotypic changes.

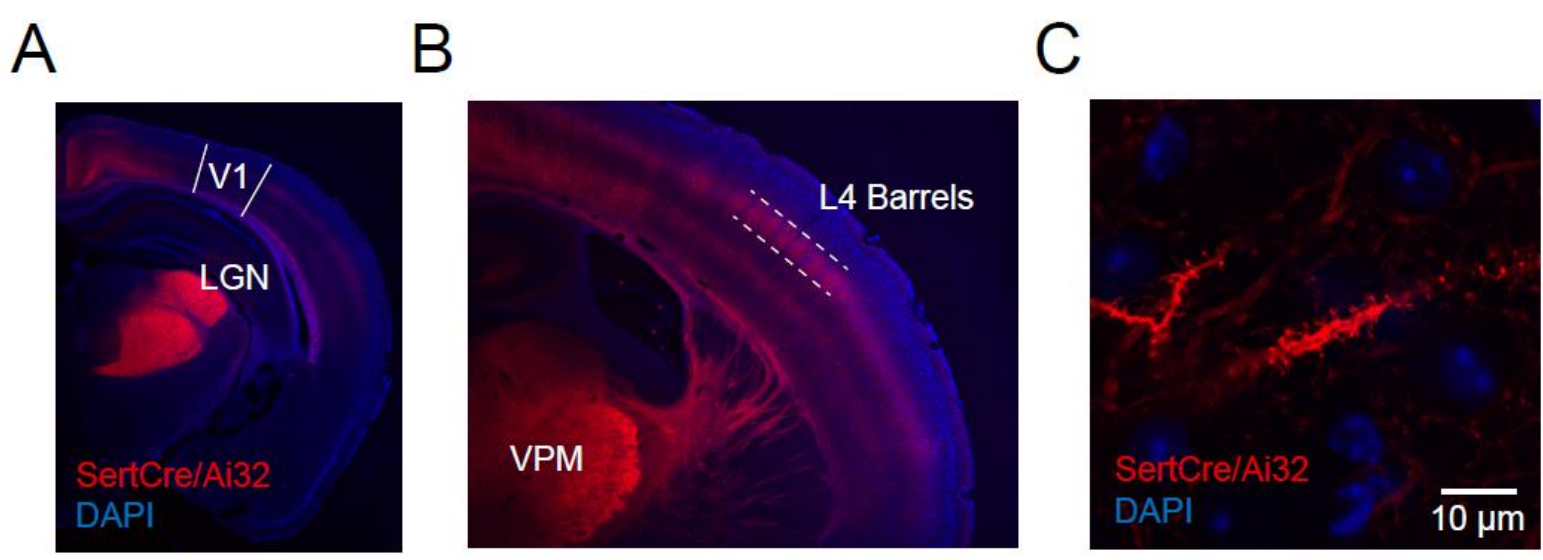

Figure 25: SERTcre driven expression of YFP-tagged channelrhodopsin. (A) and (B) Thalamic nuclei such as LGN and VPM exhibited pronounced expression of YFP-tagged channelrhodopsin in SERTcre/Ai32 mice, as expected from previous studies (Narboux-Neme et al., 2012; Narboux-Neme et al., 2008). Position of V1, as well as of $L 4$ barrels are indicated by white lines. (C) Expression of YFP-tagged channelrhodopsin was not restricted to thalamic-and brainstem nuclei, but was also prominent in a sparse population of putative layer 5 cortical cells that exhibited strong YFP signals in their dendritic processes.

\subsubsection{In-vivo whole cell recordings allow the monitoring of sensory evoked subthreshold responses}

In order to study the adaptation of subthreshold responses in cortical neurons in-vivo, I built an electrophysiological setup to perform blind whole-cell recordings in anaesthetized mice (see methods). I first established recordings in cells of upper cortical layers 2 and 3 that are typically more accessible to 
patch clamp recordings than neurons of deeper layers (Margrie et al., 2002). The recording depth was estimated for each cell by the distance by which the pipette was advanced into the tissue via the micromanipulator (Margrie et al., 2002). As shown in Fig.26 high quality recordings could be obtained as judged by low-noise levels, overshooting APs and successful biocytin labeling for post hoc identification of cells. The membrane potential of recorded cells was characterized by prominent upand-down states, whose occurrence was tightly correlated with the depth of anesthesia (data not shown).

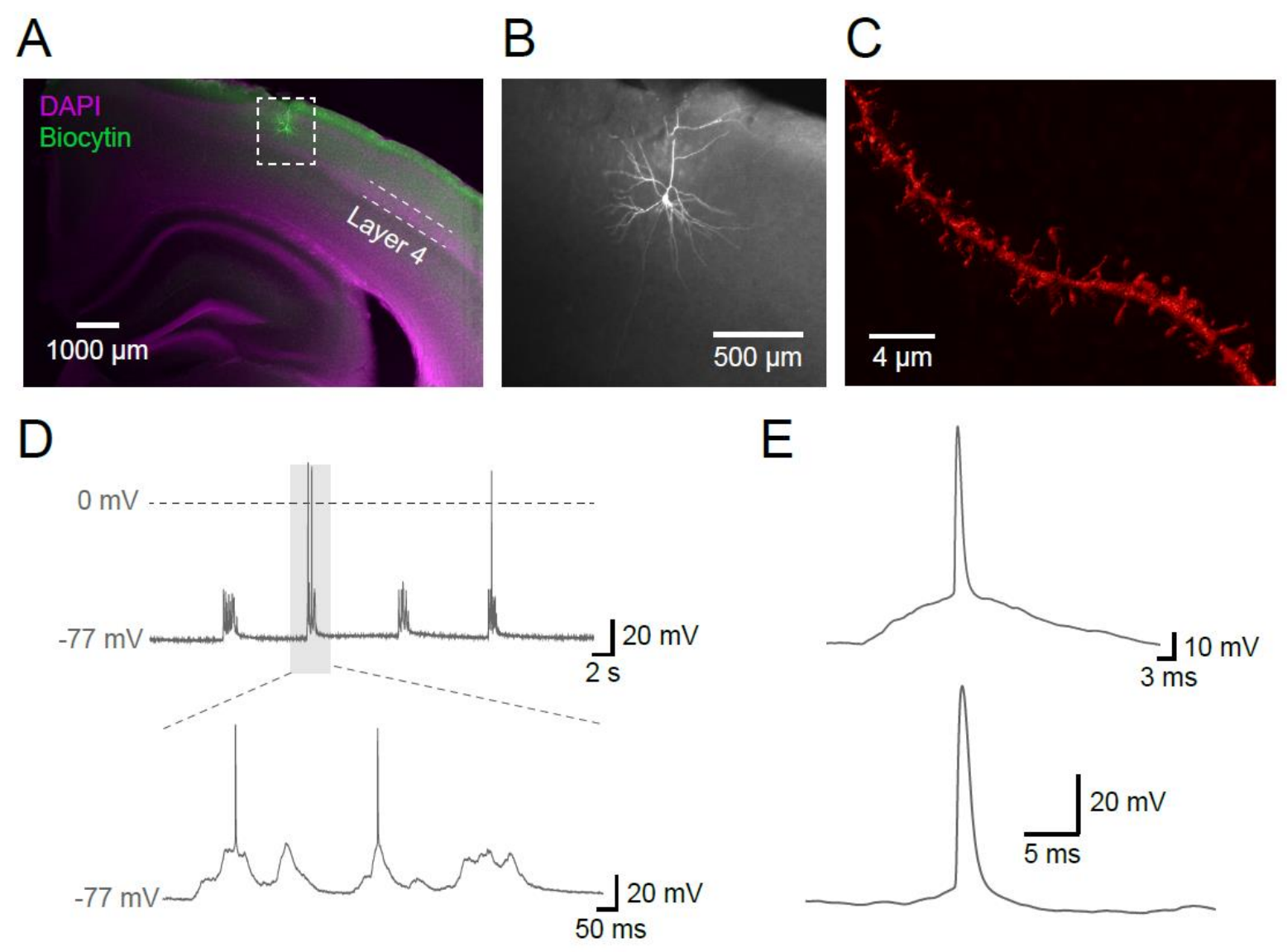

Figure 26: In-vivo whole-cell recording from morphologically identified neuron. (A) In-vivo patch clamp recordings were carried out in the upper layer of the somatosensory cortex of anaesthetized mice and biocytin was introduced for a basic morphological characterization of recorded cells. (B) Magnification of dashed box indicated in (A). The biocytin filled neuron was visualized by fluorescently coupled streptavidin. (C) Surface rendering of a dendritic segment belonging the cell illustrated in (B). The surface rendering was applied to a stack of images obtained by confocal microscopy. (D) Spontaneous activity of cell depicted in (A) and (B). Prominent up-and down states of the membrane potential were observed under isoflurane anesthesia. The gray shaded region of upper trace is magnified below. (E) Magnification of the two APs that occurred during spontaneous activity shown in (D).

Corresponding recordings were also successfully obtained in deeper layers of the cortex, but biocytin filled neurons were not recovered after measurements at these depths. Accordingly, cells were classified in subsequent experiments based on their electrophysiological responses to depolarizing currents that were previously shown to correlate with neuronal morphology (Nowak et al., 2003). 
Next, I carried out recordings in putative layer 4 neurons of the visual cortex and monitored the membrane potential, while applying visual stimuli. The stimuli consisted of repetitive 2 ms light pulses at a frequency of $4 \mathrm{~Hz}$ delivered by a blue LED lamp that was positioned at a distance of $20 \mathrm{~cm}$ to the contralateral eye (Fig.27). Results from previous studies indicated that frequencies of 4 and $4.5 \mathrm{~Hz}$, respectively, are sufficient to monitor prominent depression of synaptic responses in layer 4 cells invivo (Chung et al., 2002; Reinhold et al., 2015).

As shown in Fig.27 light pulses resulted in evoked subthreshold responses that often increased the overall firing rate of the recorded neuron. The latency of the response to the first pulse of the cell shown in Fig. 1 was $\sim 28 \mathrm{~ms}$, which is similar to what has been described in the literature for layer 4 neurons in the visual cortex of primates (Maunsell and Gibson, 1992).

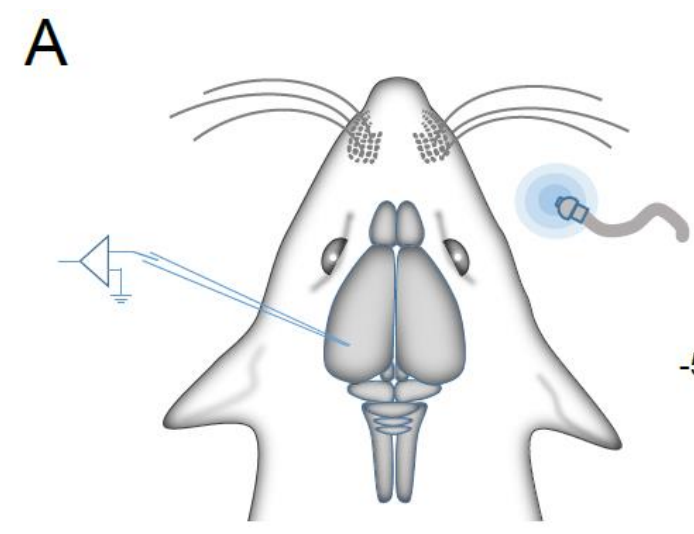

B

C
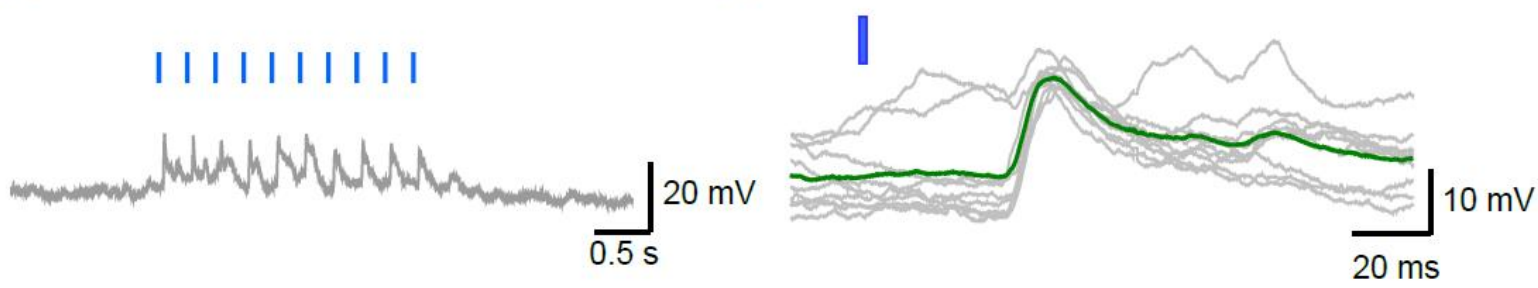

Figure 27: Light-evoked responses in a layer 4 neuron of the mouse visual cortex. (A) In-vivo patch clamp recordings were carried out in layer 4 neurons of the visual cortex of anaesthetized mice and short light pulses (2ms) were applied to the contralateral eye to measure evoked responses that are illustrated in (B). Trials were averaged and are shown in green. The blue bar indicates the stimulation period. (C) Magnification of averaged response with corresponding light pulses (blue). The responses shown in (D) were evoked by the first light stimulus.

Corresponding measurements were also carried out in $\mathrm{cCAPS1}^{\mathrm{fl} / \mathrm{wt}}, \mathrm{SERT} \mathrm{Cr} \mathrm{e}^{+/-}$mice that could potentially be used in the future as a control condition for experiments on cCAPS1 $1^{\mathrm{fl} / f l}, \mathrm{SERTcre}{ }^{+/-}$animals. Two out of three cells during these experiments were identified as regular spiking neurons based on their responses to current injections and may thus have belonged to the morphological class of spiny stellate cells in layer 4 (Fig.28A) (Nowak et al., 2003). These cells were measured in 4-5 week old animals, while 
"Cell 3" that showed very sparse spontaneous firing, as well as single- or irregular spiking upon current injection originated from a 10-week old mouse. Interestingly, light evoked responses in Cell 3 adapted more rapidly when compared to the responses of Cells 1 and 2 (Fig.28 C). Furthermore, the onset of the first response in Cell 3 exhibited a shorter latency to the first light pulse than the responses of the other two cells (Fig.28 D). Whether these variations could be ascribed to the difference of age, or cell type could not be addressed in this study.

By using the same experimental paradigm, I additionally recorded light evoked responses in a putative Layer 4 neuron from the visual cortex of a 10 -week old cCAPS1 $1^{\mathrm{fl} / f l}, \mathrm{SERTCre}^{+/-}$mouse. Based on its response to depolarizing current injections, this particular neuron was identified as a regular spiking cell and may thus have belonged to the class of layer 4 spiny stellate cells, similar to Cells 1 and 2 of the previous experiment (Fig.29 A). Interestingly, evoked responses were reliably elicited by the first light pulse, similar to Cell 3 in Fig. 28 (Fig.29 C,D). However, in contrast to all other recorded cells from CCAPS1 $^{\mathrm{fl} / \mathrm{wt}}$, SERTcre ${ }^{+-}$mice, subsequent pulses did not trigger phasic changes in the membrane potential, which instead exhibited a strikingly asynchronous state (Fig.29, C).

Taken together, these preliminary results suggest that this experimental systems holds the potential to successfully be applied in the future to address the effect of presynaptic perturbation on sensory processing. 
A

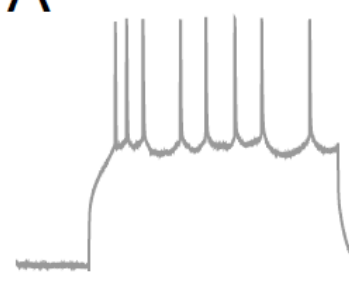

$\frac{\int_{0.1 \mathrm{~s}}}{30 \mathrm{mV}}$

B

Cell 1

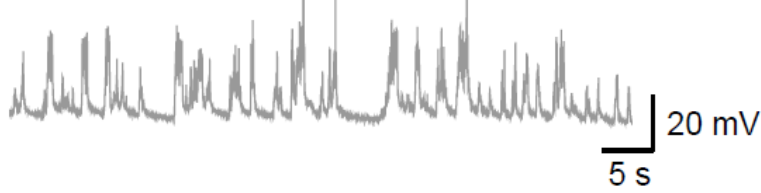

Cell 2

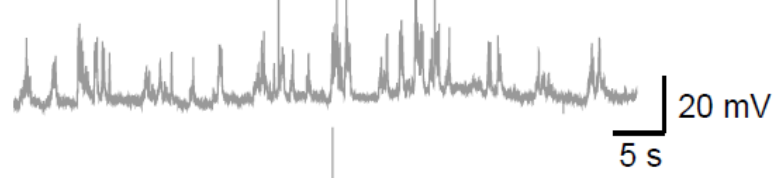

Cell 3

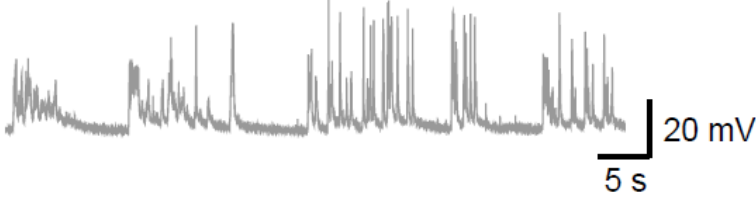

C

D

Cell 1

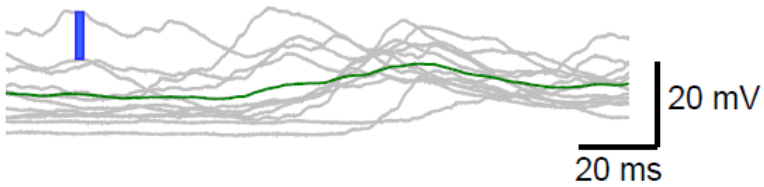

Cell 2

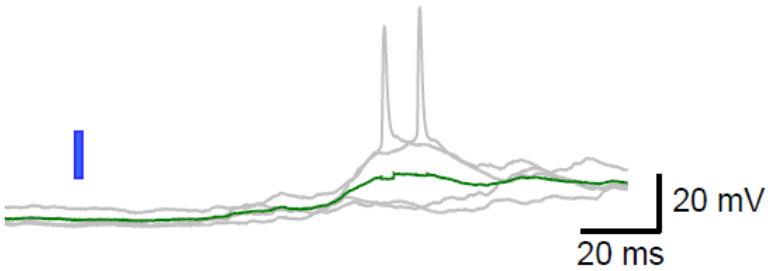

$\underbrace{}_{0.5 \mathrm{~s}} 20 \mathrm{mV}$
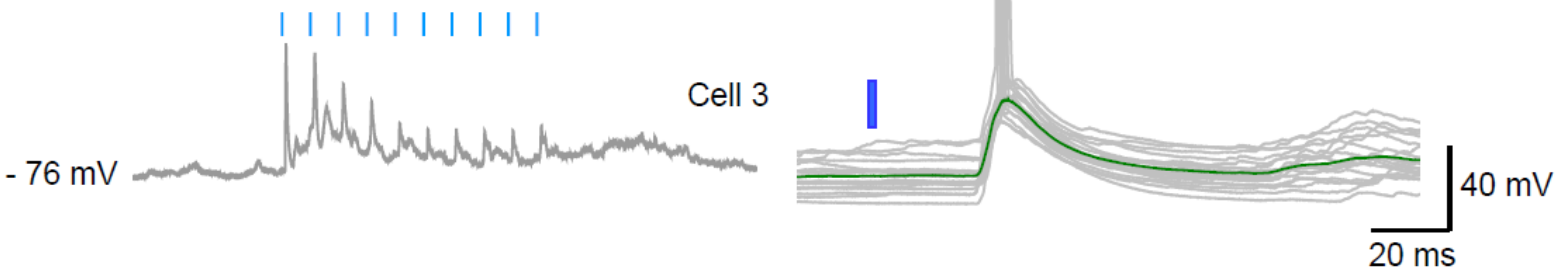

Figure 28: Basic electrophysiological analysis of Layer 4 neurons from the visual cortex of CAPS1 ${ }^{f / w t}$, SERTcre mice. (A) Firing properties of neurons were studied by injecting current pulses of $400 \mathrm{pA}$ and by monitoring the spontaneous activity (B). (C) Averaged light evoked responses of cells shown in (A) and (B). The blue bars indicate time points of light pulses. (D) Raw traces and averaged responses obtained for the first light evoked pulse. 
A

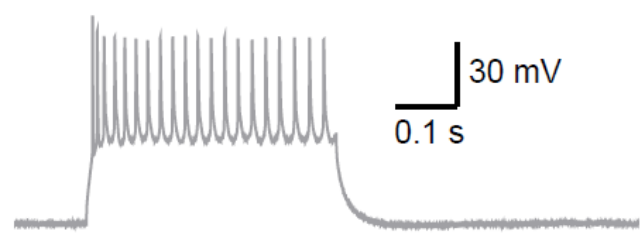

C $\quad \begin{aligned} & \text { cCAPS1 } 1 / f l \\ & \text { Sert Cre }+/-\end{aligned}$
B

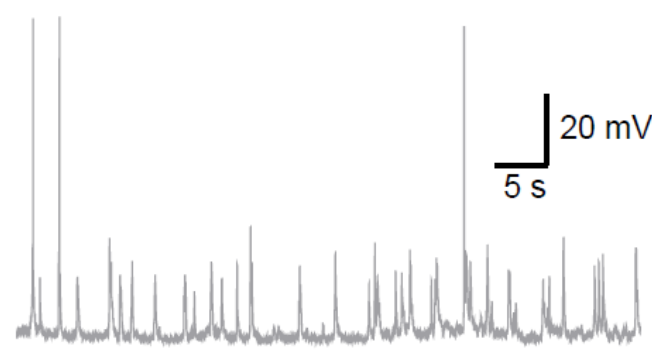

D

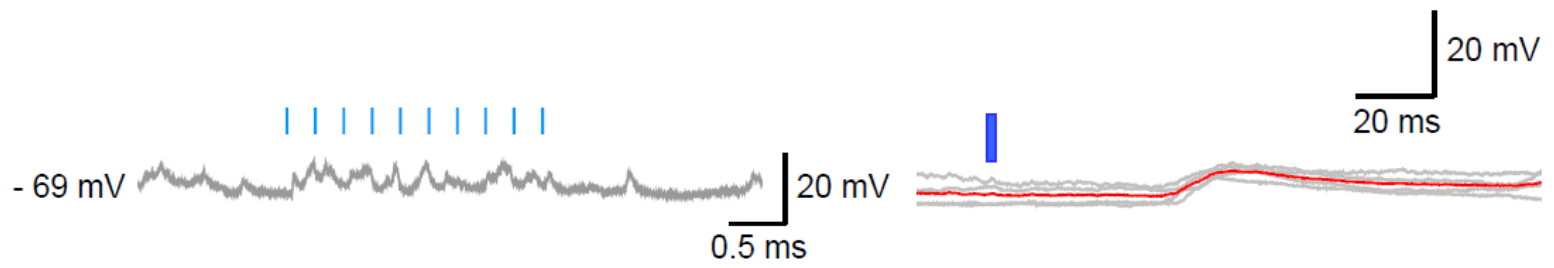

Figure 29: Basic electrophysiological analysis of a Layer 4 neuron from the visual cortex of a CAPS1 ${ }^{\text {flffl}}$, SERTCre mouse. (A) Firing properties of the neuron were studied by injecting a current pulses of $400 \mathrm{pA}$ and by monitoring the spontaneous activity (B). (C) Averaged light evoked responses of cell shown in (A) and (B). The blue bars indicate time points of light pulses. $(D)$ Raw traces and averaged response obtained for the first light evoked pulse. 


\section{Discussion}

\subsection{Overview}

Virtually all brain functions including motor coordination and sensory processing require the temporally precise release of transmitter in response to APs. Before AP-triggered release, SVs are recruited to the plasma membrane and undergo a series of reactions to become fusion competent. These reactions, referred to as priming, are orchestrated by a number of proteins. Some of these are essential and form the core of the presynaptic release machinery, while others are functioning as modulators and fine tuners. Despite much progress in the last two decades in identifying many of the proteins that regulate the fusion competence and release probabilities of SVs, the priming process as such is still poorly understood.

In the present study I aimed at functionally dissecting the SV priming process in greater detail and to establish an experimental system that would allow the future analysis of the significance of this process for STP phenomena in the context of visual information processing.

In a first set of experiments, I addressed the functional role of two recently identified presynaptic proteins in the regulation of the SV priming process. For this, I studied synaptic transmission in cultured neurons from the hippocampus of SYD1- and Mover deficient mice. My results revealed that neither these proteins regulate transmitter release in cultured cells, in contrast to what has been suggested by other studies using acute slice preparations.

In addition, I functionally dissected the interplay between the two known essential priming factors CAPSs and Munc13s. By carrying out a structure function analysis, I found that CAPSs do not require their SNARE-binding MUN domain to establish a pool of primed vesicles for basic transmitter release. Rather, my results suggest that CAPSs prime SVs via their $\mathrm{PIP}_{2}$ binding $\mathrm{PH}$ domain and that this functional requirement can be by-passed by an activity dependent mechanism that involves the DAG-binding C1 domain of Munc13s. Further results of the structure function study revealed that a subset of BPDassociated mutations identified in the human homologue of CAPS1 lead to altered STP, indicating that perturbations in the priming process may contribute to the biogenesis of affective disorders in patients.

In order to establish an experimental system for the future study of STP phenomena in the context of visual information processing, I generated a new mutant mouse strain, in which CAPS1 proteins are deleted in thalamic nuclei and built an electrophysiological setup to record sensory evoked responses in layer 4 neurons of the visual cortex of anaesthetized mice. 


\subsection{Proteins of the mSYD1 family are not involved in modulating transmitter release in cultured autaptic neurons}

A recent study identified mSYD1A as a presynaptic protein that interacts with the active zone components Munc18 and $\alpha$-liprin-2 (Wentzel et al., 2013). Based on its domain structure and protein sequence it was speculated to constitute a distantly related mammalian orthologue of the invertebrate SYD1 protein that was previously shown to modulate synapse assembly and to regulate the shape, size and composition of active zones (Dai et al., 2006; Hallam et al., 2002; Owald et al., 2010; Patel et al., 2006). Genetic removal of mSYD1A in mice was reported to lead to severe reductions in the number of docked vesicles and in the frequency of spontaneous mEPSCs in acute hippocampal slices (Wentzel et al., 2013). Interestingly, these deficits were not accompanied by changes in synapse density, or active zone length indicating that mSYD1A may perform functions in mammals that are distinct from that of SYD1 in invertebrates.

In order to address these functions in greater detail, I studied transmitter release in cultured autaptic cells by measuring postsynaptic currents. Based on previous findings of a reduction in the number of docked vesicles, I expected to find corresponding changes in the number of primed vesicles (Imig et al., 2014; Murthy et al., 2001; Schikorski and Stevens, 2001; Siksou et al., 2009). Surprisingly, however, I neither observed differences in AP- and sucrose evoked responses, nor in the frequency of mEPSCs between mSYD1A KO- and WT neurons. Furthermore, application of trains of APs did not reveal any changes in transmitter release during enhanced presynaptic activity, indicating that synaptic transmission in cultured autaptic neurons is not perturbed upon deletion of mSYD1A.

In order to exclude the possibility of a compensatory mechanism mediated by another mSYD1 isoform, I studied synaptic transmission in mSYD1 A,B DKO neurons. Again, I neither found differences in basic synaptic transmission, nor in the activity dependent modulation of transmitter release, indicating that mSYD1s are dispensable for the proper functioning of the presynaptic release machinery in cultured autaptic neurons.

What are possible explanations for the discrepancy between results of this study and previous findings? First, the use of cultured autaptic cells may have obscured the previously published phenotype. This argument may be supported by a previous study in which deletion of the presynaptic protein synaptotagmin-1 led to a reduction in sucrose triggered EPSCs in neuronal mass cultures, while RRP sizes remained unchanged in single autaptic neurons (Liu et al., 2009). In further support of this hypothesis, KD of mSYD1A in cerebellar mass cultures was reported to lead to a similar reduction in mEPSC frequency as observed for acute brain slices from the hippocampus of KO mice (Wentzel et al., 
2013). Thus, future studies are required to test this hypothesis in more detail by studying synaptic transmission in mass cultures prepared from KO mice.

A second possible explanation for the discrepancy between the documented reduction in the number of docked vesicles and the findings of indifferent RRP sizes may be given by assuming that morphological docking and functional priming may not reflect the same cell biological process (Kaeser and Regehr, 2017). According to this assumption, the application of sucrose pulses may trigger the uniform release of a heterogeneous population of primed SVs that differ from one another in their initial docking state, while exhibiting the same release probabilities in KO- and WT cells. Shifting the balance of the fraction of SVs that are docked in favor of the fraction of vesicles that are undocked, however, should lead to some degree to changes in synaptic transmission, as suggested by previous studies (FernandezBusnadiego et al., 2013; Han et al., 2011; Imig et al., 2014; Siksou et al., 2009; Toonen et al., 2006; Wang et al., 2016). Thus, since no changes in synaptic transmission could be detected, it is unlikely that deficits in the docking of vesicles have occurred under the experimental conditions that were used in this study.

A third possible explanation of the discrepancy relates to the technical approach by which the docking state of SVs was defined in the previous mSYD1A study. Classically, SV docking has been studied by analyzing 2D images obtained by EM on chemically fixed tissue (Jockusch et al., 2007; Schoch et al., 2002; Schoch et al., 2006; Toonen et al., 2006; Varoqueaux et al., 2002; Wentzel et al., 2013). In recent years, however, 3D electron tomography applied to high-pressure frozen tissue samples has proven to yield a much more accurate description of the docked state that closely correlates with RRP measurements in cultured autaptic neurons (Fernandez-Busnadiego et al., 2013; Imig et al., 2014; Siksou et al., 2009). Furthermore, docking can be distinguished in 3D tomography from other cell biological processes such as tethering, thereby enabling a highly differentiated analysis of sub population within the overall SV pool (Fernandez-Busnadiego et al., 2010). Accordingly, by assuming a specific effect of SYD1A on a subset of vesicles it is possible that these may differentially contribute to the docked pool in conventional 2D EM versus 3D tomography. Even though this hypothesis may possibly explain the discrepancy between the previously published docking deficit and the RRP results of this study, it cannot be applied to addressing the different findings of changes in mEPSC frequency (Wentzel et al., 2013).

In conclusion, more studies are required to test the relevance of mSYD1 in synaptic transmission. These studies should include the electrophysiological analysis of synaptic transmission in mass cultured cells and should further apply 3D tomography on high pressure frozen tissue samples to obtain a more differentiated view on the docked state of SVs. 


\subsection{The presynaptic protein Mover does not regulate release probability in cultured autaptic neurons}

A small number of presynaptic proteins have emerged in the course of evolution including synuclein, bassoon and Mover that are uniquely expressed in vertebrates (Ahmed et al., 2013; Kremer et al., 2007). Among these, Mover is the most recently identified protein and the only information available on its function is based on one study in which KD experiments were performed in the calyx of Held synapse (Korber et al., 2015). Results of this particular study suggested that Mover may act as a modulator of transmitter release by regulating the probability by which SVs fuse during APs (Korber et al., 2015). In order to address this function in greater detail, I studied synaptic transmission in cultured autaptic cells derived from the hippocampus of Mover KO mice.

Interestingly, in contrast to the KD study, I neither observed changes in the EPSC amplitude nor in STP characteristics. Furthermore, all of the other parameters analyzed, including RRP size and mEPSC frequency, did not reveal any changes upon deletion of Mover.

At present it remains unclear why Mover KD in the calyx of Held and Mover KO in autaptic hippocampal cells have led to different electrophysiological results. One possible explanation of this may be related to the technical approach by which the protein level was either fully or incompletely depleted by KO or $K D$, respectively. Interestingly, previous studies have shown that KD versus KO strategies in studying the role of presynaptic proteins (e.g. complexins) can lead to different findings (Yang et al., 2013). To test this hypothesis in the future, synaptic transmission could either be studied in Mover KD neurons in autaptic cultures, or in calyx of held synapses from Mover KO mice.

Alternatively, differences in findings between published results and the data of this study may have originated from differences in the model systems that were used to study synaptic transmission. The calyx of Held synapse is a highly specialized synapse with many active zones that is optimized to sustain transmitter release at a high frequencies (Borst and Soria van Hoeve, 2012; Neher, 2017; Schneggenburger and Forsythe, 2006; von Gersdorff and Borst, 2002). In contrast, glutamatergic synapses of cultured hippocampal neurons typically harbor one to very few active zones and the firing of APs in excitatory hippocampal neurons in-vivo does not match the high frequency level present in the calyx of Held synapse (Kandel and Spencer, 1961; Lorteije et al., 2009; Schikorski and Stevens, 1997). Accordingly, Mover may play a modulatory role in the Calyx of Held synapse to sustain transmitter release at high frequency levels, while its function in hippocampal neurons may either not be directly related to transmitter release or its loss may be compensated by other proteins that are not present in the calyx of Held synapse. 


\subsection{SV priming in absence of CAPS proteins requires the C1 domain of Munc13}

Munc13- and CAPS proteins were identified in the past to act as essential SV priming factors (Jockusch et al., 2007; Varoqueaux et al., 2002). Despite their identification, it is largely unknown how these two protein families functionally cooperate to render SV fusion competent. In one study it was shown that the essential requirement of CAPSs for transmitter release can functionally be by-passed by HFS or application of PDBu (Jockusch et al., 2007). Since the activity of Munc13s is known to be regulated by $\mathrm{Ca}^{2+}$ and DAG/PDBu binding, it has been hypothesized that the loss of CAPSs during priming may be compensated by these proteins. In the present study, I tested this hypothesis by characterizing the residual priming activity in CAPS-deficient neurons in greater detail.

In a first set of experiments, I was able to confirm the previously published rescue of AP-evoked EPSCS by PDBu in CAPS DKO neurons (Jockusch et al., 2007). In addition, I observed larger sucrose evoked EPSCS in PDBu treated than in untreated CAPS DKO neurons indicating that PDBu rescues transmitter release by increasing the number of primed vesicles. Interestingly, the capacity of PDBu to increase the number of primed vesicles is discussed controversially in the literature. Some studies have reported an increase in sucrose-triggered EPSCs in WT neurons, while others have only observed prominent changes in AP-evoked EPSCs (Basu et al., 2007; Stevens and Sullivan, 1998). The discrepancy in findings are presently thought to have originated from differences in the mode of sucrose application to cultured cells in these studies for RRP measurements (Basu et al., 2007; Stevens and Sullivan, 1998). Specifically, one study reported that PDBu may lower the energy barrier for fusion resulting in larger currents upon suboptimal sucrose stimulation ( $250 \mathrm{mM}$ ), while under maximal tonicity levels (500 mM sucrose) an equal amount of RRP vesicles can be released from untreated-and treated neurons, respectively (Basu et al., 2007). In the present study, I applied 500 mM sucrose solution using a fast flow application system that in contrast to microinjection devices is thought to not cause significant tonicity gradients along the dendritic tree of stimulated cells (Trimbuch and Rosenmund, 2016). By using this approach, I was able to clearly distinguish between two separate components in sucrose evoked EPSCs that reflected the fusion of RRP vesicles and the fusion of vesicles that were newly recruited during the pulse. Thus, I concluded that the increase in RRP size during my measurements was caused by a higher number of primed vesicles and did not result from changes in the fusogenicity state of the few vesicles that were primed before PDBu application, or from vesicles that were newly recruited during sucrose application. Furthermore, I observed a higher normalized steady-state EPSC amplitude after HFS in treated than in untreated CAPS DKO cells, supporting the hypothesis that PDBu increases the activity level of SV priming in CAPS-deficient cells. 
In order to test, whether activation of Munc13s may underlie the residual priming activity in CAPS DKO neurons, I studied synaptic transmission in CAPS deficient cells that additionally contained the H567K mutation in the DAG/PDBu binding C1 domain of Munc13-1 and did not express Munc13-2 (in previous and following sections referred to as CAKI neurons).

Strikingly, a detailed analysis of synaptic transmission in CAKI cells revealed a strong impairment of transmitter release that even exceeded the previously published CAPS DKO phenotype in the degree of severity (Jockusch et al., 2007). Notably, neither HFS nor PDBu application could rescue transmitter release in these neurons in contrast to what has been reported for CAPS DKO cells.

A number of conclusions can be drawn from these results. First, the H567K mutation in the $\mathrm{C} 1$ domain of Munc13-1 abolishes SV priming in absence of CAPS proteins. Accordingly, Munc13-1 ${ }^{\text {H567K }}$ does unambiguously constitute a loss-of-function mutant protein in the context of CAPS deficiency. This finding in turn offers key insights into functional nature of the H567K mutation, which has been studied extensively in the past years. Initially, the H567K mutant was described to constitute a loss-of-function protein which impairs priming as suggested by a reduction in the RRP size and a failure of PDBu-induced EPSC potentiation in cultured Munc13-1 ${ }^{\mathrm{H} 567 \mathrm{~K}}$ neurons. Later, however, it was reported that sucrose pulses at submaximal tonicity levels lead to larger EPSC charges in mutant than in control neurons suggesting that the energy barrier for fusion is reduced by the mutation (Basu et al., 2005). In addition, some studies reported an increase of the mEPSC frequency in cultured cells and in neurons from organotypic sclices prepared from the hippocampus of Munc13-1 $1^{\mathrm{H} 567 \mathrm{~K}}$ mice, similarly to PDBu treatment, thus corroborating the hypothesis of a lowered energy barrier (Basu et al., 2007; Lou et al., 2008). Collectively, these results led to the hypothesis that Munc13-1 $1^{\mathrm{H} 567 \mathrm{~K}}$ is a gain of function mutant that conformationally mimics the DAG-bound state (Basu et al., 2007). Based on this more recent hypothesis, I initially expected that expression of Munc13-1 ${ }^{\mathrm{H} 567 \mathrm{~K}}$ in CAPS deficient cells would mimic PDBu treatment and would thus rescue EPSC amplitudes. Yet, I observed that transmitter release in CAKI cells was even more perturbed than in CAPS DKO neurons. A possible explanation for this may be given by postulating a dual function of Munc13s in transmitter release, by regulating priming and fusion as recently suggested (Xu et al., 2017). Based on this assumption, introduction of the H567K mutation may on one hand perturb an essential activity dependent priming process, while on the other hand leading to an energetically more favorable fusion state of SVs (Basu et al., 2007; Rhee et al., 2002; Xu et al., 2017). This model would also explain why submaximal sucrose pulses trigger the release of a relatively higher fraction on SVs in Munc13-1 ${ }^{\text {H567K }}$ neurons than in controls, while the overall RRP size is reduced in mutant cells when EPSC charges are measured by sucrose stimuli at maximal tonicity (Basu et al., 2007; Rhee et al., 2002). 
Second, the results support the previously published hypothesis that Munc13s are functionally involved in compensating for a loss of CAPSs at high presynaptic activity. This in turn indicates that CAPSs and Munc13s functionally cooperate during the SV priming process.

Lastly, since other domains of Munc13-1 that are known to bind $\mathrm{Ca}^{2+}, \mathrm{PIP}_{2}$ and calmodulin were not mutated in this study and are therefore expected to have structurally been left intact, it is highly likely that activation of these by the respective second messengers was not sufficient to establish transmitter release in CAKI cells. This in turn indicates that the $\mathrm{C} 1$ domain of Munc13s may play a rather crucial role during activity dependent priming, while other regulatory domains in Munc13s may be involved in modulating different aspects of activity-dependent release. Alternatively, the H567K mutation may not only have affected the function of the C1 domain of Munc13-1, but may in addition have perturbed the function of other protein regions. Interestingly, a recent study reported a functional synergy between the $\mathrm{C} 1, \mathrm{C} 2 \mathrm{~B}$ and $\mathrm{C} 2 \mathrm{C}$ domains in sustaining the action of Munc13s during priming, indicating that dysfunction of one of these domains may negatively affect the combined impact that these domains have on sustaining transmitter release (Liu et al., 2016). Additionally, it was recently mentioned in one study that the H567K mutation may negatively affect the function of the RIM binding C2A domain that is required for the recruitment of Munc13s to the active zone (Betz et al., 2001; Deng et al., 2011; Dulubova et al., 2005; Xu et al., 2017). Even though the authors of that study did not provide results to support their statement, a few prior observations may support their argument. First, removal of the C2A domain was shown before to reduce AP- and sucrose evoked EPSCS in cultured neurons by $50 \%$ when compared to full-length Munc13-1 expressing cells (Liu et al., 2016). In contrast, introduction of the H567K mutation into the $\mathrm{C} 1$ domain of Munc13s only leads to corresponding changes in sucrose evoked charges, while AP-evoked EPSCs remain unchanged (Rhee et al., 2002). Thus, based on the model of a dual effect of the H567K mutation on priming and on regulating the energy barrier for fusion, EPSC amplitudes in H567K mutant expressing cells may reach WT-levels despite C2A domain loss of function due to an enhanced fusogenicity state of SVs whose priming is independent of the C2A domain. To test this hypothesis in the future, $\mathrm{C} 2 \mathrm{~A}$-domain lacking Munc13-1 ${ }^{\mathrm{H} 567 \mathrm{~K}}$ could be used for rescue experiments in cultured Munc13-DKO neurons and AP-evoked EPSCs could be compared to those of C2A-domain lacking Munc13-1 ${ }^{\mathrm{WT}}$ expressing cells.

\subsection{Analysis of the structure function relationship of CAPS proteins reveals key insights into the SV priming process}

Several studies in the past have addressed the structure-function relationship of Munc13s in glutamatergic synaptic transmission yielding key insights into the priming process. Corresponding 
studies, however, have not been carried out for CAPS proteins. An earlier study demonstrated that loss of either one of the two priming factors cannot be compensated by cross-rescue experiments, suggesting that CAPSs and Munc13s either act in series, or jointly in the same molecular pathway (Jockusch et al., 2007). Accordingly, by carrying out a structure function study of CAPS proteins, I not only expected to learn more about the contribution of each of the domains in CAPSs to the priming process, but further, I expected to gain key insights into the non-redundant functions of Munc13s and CAPSS.

Results from rescue experiments in this study on cultured CAPS DKO neurons revealed that CAPS essentially requires its central $\mathrm{PIP}_{2}$ interacting $\mathrm{PH}$ domain in order to promote $\mathrm{SV}$ priming. Furthermore, other domains including the SNARE-interacting MUN domain, the C-terminal DCV binding site and a conserved region within the DI domain were found to be dispensable for the function of CAPS to sustain AP-evoked release at basic activity levels. Key findings of these results are discussed in the following three sections

\subsubsection{The C-terminal half of CAPS is not essential to sustain basic synaptic transmission}

Previous studies suggested an essential role of the MUN domain and the DCV binding site of CAPSs in supporting peptide release from PC12 cells and cultured c.elegans neurons (Grishanin et al., 2002; Khodthong et al., 2011; Lin et al., 2010; Parsaud et al., 2013). In order to test this essential role in glutamatergic synaptic transmission, I overexpressed the CAPS2e splice variant, which lacks the MUN domain and DCV-binding site, and studied its rescue capacity in CAPS DKO neurons, comparing it to fulllength CAPS2b. As shown above, AP-evoked responses were equally rescued by both splice variants indicating that neither the MUN domain nor the DCV binding site are essential to sustain basic synaptic transmission. In contrast, however, RRP sizes tended to be smaller by a factor of 2 in CAPS2e expressing neurons and EPSCs depressed much more rapidly during trains of APs. As briefly described in the results section of this study, I interpreted these findings by postulating a selective role of the C-terminal half of CAPSs in establishing an additional number of primed vesicles that may not fuse in response to single APs, but may rather release their contents into the synaptic cleft during repetitive stimulation. This interpretation is supported by previous studies, which reported that the RRP consists of a heterogeneous population of SVs that differ from one another in their release probabilities (Neher, 2015). The N-terminal half of CAPSs may thus be sufficient to prime a sub pool of RRP vesicles that is characterized by a high release probability and is required to sustain AP-evoked release at basic conditions. Further experimental support of this assumption is discussed in section 4.6. 
Notably, a modulatory rather than an essential role of the C-terminal half of CAPSs in transmitter release was also observed by our collaborator in corresponding overexpression experiments in adrenal chromaffin cells (Nguyen et al., 2014). There, expression of CAPS2e led to a partial rescue of the dramatic RRP deficit in CAPS DKO cells derived from the adrenal gland of mice (Nguyen Truong et al., 2014). Thus, the discrepancy between results of this study and previously published results on the essential role of the MUN domain, as well as of the DCV binding site, cannot be explained by a differential requirement of the C-terminal half for SV-versus DCV priming. Furthermore, it can also not be explained by overexpression artifacts, since all of the previous studies that have addressed the structure-function relationship of CAPSs in neuronal secretion by intact cultured cells strongly expressed CAPS cDNA constructs using overexpression systems as well (Kabachinski et al., 2016; Khodthong et al., 2011; Lin et al., 2010). Rather, differences in the findings may have originated from differences in the sensitivity of assays applied for the monitoring of release or from the usage of primary cell cultures, instead of stable cell lines, such as PC12 cells, or permeabilized ghost cells (Grishanin et al., 2002; Kabachinski et al., 2016; Khodthong et al., 2011; Lin et al., 2010). An essential function of the C-terminal half of CAPS may, however, not be entirely excluded under physiological expression levels. Interestingly, expression of the CAPS2e splice variant via lenti viruses that typically yields expression levels comparable to those of endogenous proteins, did not rescue AP-triggered release in CAPS DKO neurons (supplementary Figure 8). This in turn may be explained by assuming that the C-terminal half of CAPSs may be involved in targeting the protein to release sites, while the $\mathrm{N}$-terminal half harbors the catalytically essential activity required for the priming process. To test this hypothesis, future studies could analyze whether CAPS2e is correctly trafficked to presynaptic structures at physiological expression levels.

\subsection{2. $\quad$ SV Priming requires the $\mathrm{PIP}_{2}$ binding $\mathrm{PH}$ domain of CAPSS}

The $\mathrm{PIP}_{2}$-binding PH domain of CAPSs has been described in the literature to be essential for the release of transmitter from DCVs in permeabilized PC12 cells and to further be required for the acceleration of SNARE-dependent liposome fusion by CAPS (Grishanin et al., 2002; James et al., 2008). In the present study, I was able to additionally confirm a crucial involvement of the $\mathrm{PH}$ domain in transmitter release by glutamatergic neurons. As shown above, expression of CAPS2c, which harbors a deletion within the $\mathrm{PH}$ domain, did not rescue transmitter release in cultured CAPS DKO neurons in contrast to full-length CAPS $2 b$. Furthermore, introduction of a triple-point mutation in the PH domain of CAPS2e, which is expected to abolish $\mathrm{PIP}_{2}$ binding, led to a similar pronounced deficit in transmitter release. Together, these results suggest that PIP 2 binding by the PH domain of CAPSs is essential to sustain SV priming. Specifically, since the mutated CAPS2e protein was strongly overexpressed by Semliki Forest viruses, it 
is unlikely that PIP 2 binding is solely required to recruit CAPSs to release sites. Instead, it is likely catalytically involved in the formation of a primed pool of vesicles. This argument is supported the observation that expression of CAPS2e at physiological levels does not rescue transmitter release in DKO cells while overexpression does, indicating that potential deficits in targeting the protein to release sites can be compensated by strong overexpression. Interestingly, Semliki Forest virus mediated overexpression has before been reported to compensate for deficits in AP-evoked EPSC amplitudes, thus further validating the argument of a catalytically essential function of the PH domain (Basu et al., 2005; Betz et al., 2001).

What may be a potential mechanism for the catalytic activity of the PH domain during priming? Based on previous models that CAPSs may tether vesicles to release sites, $\mathrm{PIP}_{2}$ binding may be required to bridge the distance between SVs and the plasma membrane (Grishanin et al., 2002; Lin et al., 2010). Interestingly, a function of CAPSs in tethering was also recently proposed by another study that employed 3D electron tomography to study SV docking in CAPS- and Munc13 deficient cells, respectively (Imig et al., 2014). There, the authors demonstrated a similarly pronounced docking deficit for both experimental conditions, while SVs were located at a further distance from the active zone in CAPS than in Munc13 deficient cells (Imig et al., 2014). This observation led the authors to propose that CAPSs may be involved in tethering SVs to the active zone before these are docked by downstream processes. However, the applicability of this model to the interpretation of the data presented here is complicated by prior studies, which reported an involvement of the DCV binding site of CAPSs in tethering vesicles to the plasma membrane (Grishanin et al., 2002; Lin et al., 2010). Alternatively, since CAPSs are known to act as dimers, one possible mechanism by which the CAPS2e splice variant may bridge the two membranes could be provided by binding acidic phospholipids on the SV surface and by binding $\mathrm{PIP}_{2}$ at the plasma membrane via two PH domains (Petrie et al., 2016). Although this speculative model may explain some of the key findings described above, experimental evidence is sparse and future studies are required to test its validity.

\subsubsection{Deletion of a conserved stretch of amino acids within the DI domain of CAPS2e does not perturb synaptic transmission}

The results discussed in the previous two sections strongly argue in favor of an essential role of the $\mathrm{N}$ terminal half of CAPS2 in establishing a pool of SVs for basic transmitter release. In addition to its PH domain, the N-terminal half of CAPSs contains a C2- and a DID domain, whose exact functions in glutamatergic synaptic transmission have not been addressed. In the present study, I aimed at studying the function of the DI domain. 
Partial deletion of the DI domain has previously been shown to impair BDNF release by PC12 cells or cultured hippocampal neurons respectively, and to further lead to motor impairments in c.elegans worms (Sadakata et al., 2012; Sadakata et al., 2007b; Speese et al., 2007). Based on these findings, I speculated that the DI domain may be required for the CAPS dependent priming process in glutamatergic neurons. To test this hypothesis, I deleted a conserved stretch of amino acids within the DI domain of CAPS2e ("WIAKY") and tested its effect on synaptic transmission in cultured hippocampal cells. Interestingly, my results did not reveal any differences in transmitter release between mutant CAPS2 $e^{\text {WIAKY }}$ and CAPS2 $e^{\text {WT }}$ expressing DKO cells. Two possible scenarios may explain this surprising outcome of these results. First, other studies in the past that described an essential requirement of the DI domain in BDNF release and locomotion of c.elegans worms analyzed mutant proteins that contained larger deletions (Sadakata et al., 2007a; Speese et al., 2007). Thus, the simplest explanation may be given by speculating that other conserved protein stretches within the DI domain are more important for protein function than the region that I deleted for the experiments described above. Alternatively, strong overexpression of the mutant protein may have masked potential deficits in regulatory functions of the domain during my experiments. While this hypothesis may be applicable to explain why potential deficits in mislocalization of the protein did not become manifest as perturbations in transmitter release during my experiments, it does not explain why others observed a deficit in BDNF release in mutant overexpressing PC12 cells (Sadakata et al., 2007b). Accordingly, future studies are required to further dissect the role of the DI domain of CAPSs in transmitter release. For this, larger deletions could either be introduced into CAPS2e, resembling those of previous studies, or mutant full-length CAPS2 ${ }^{\text {WIAKY }}$ may be expressed at physiological expression levels for rescue experiments in CAPS DKO neurons.

\subsubsection{BPD associated mutations in CADPS1 change the degree of STD in cultured neurons}

Further insights into the structure function relationship of CAPSs were provided by rescue experiments in cultured CAPS DKO neurons using eight different CADPS1 CDNA constructs that carried mutations found in BPD patients. I found that all of the mutant proteins rescued AP- and sucrose evoked EPSCS equally well. A detailed analysis of EPSCs during trains of APs, however, revealed that two of the mutations led to striking changes in the degree by which amplitudes adapt to prolonged stimulation. Specifically, expression of the C2 domain mutant S399L induced less prominent STD in cultured neurons during early phases of AP trains and the activity dependent replenishment rate of RRP vesicles was found to be higher than in control neurons. In contrast, EPSCS of DI domain mutant CADPS1 ${ }^{\text {dEX2 }}$ cells exhibited a more pronounced depression of their amplitude during trains, while the activity-dependent replenishment rate was found to not be different from that of control cells. Furthermore, the frequency 
of mEPSCS as well as the vesicular release probability were found to be increased by the CADPS1 ${ }^{\mathrm{dEX} 2}$ mutation, contrary to CAPDS1 $1^{\text {S399L }}$ which did not induce any of these changes when expressed in cultured neurons. Thus, although both mutations led to changes in the degree by which neurons adapt their responses to prolonged stimulation, the underlying cell biological mechanisms by which these changes were caused may have differed between the two mutations.

In detail, the increase in vesicular release probability, possibly resulting from the insignificantly smaller RRP size in CADPS1 ${ }^{\mathrm{dEX} 2}$ cells, as well as the higher mEPSC frequency may indicate that the CADPS1 ${ }^{\mathrm{dEX} 2}$ mutation may affect the fusion state of RRP vesicles. The mechanism by which CAPSs could regulate the fusion state, however, remains elusive at the present time. One possibility may be given by assuming that CAPSs is involved in stabilizing a subset of primed vesicles (see section 3.6). Interference with this function, in turn, may lead to premature fusion, which becomes manifest as an increased spontaneous fusion rate and an increase in $\mathrm{Ca}^{2+}$ sensitivity for transmitter release. Although this possible scenario may explain the increased mEPSC frequency as well as the more pronounced STD in CADPS1 ${ }^{\mathrm{dEX} 2}$ mutant cells, it is limited by the observation that AP-evoked responses at rest are not increased by the mutation. Such an increase, nonetheless, would be expected as a consequence of a higher $\mathrm{Ca}^{2+}$ sensitivity, as suggested by previously published findings (Korber et al., 2015; Lou et al., 2008). Thus, future experiments may shed more light on the functional mechanism by which the dEX2 mutation exerts its effect on synaptic transmission. In these experiments, the $\mathrm{Ca}^{2+}$ sensitivity of release may be studied by varying extracellular $\mathrm{Ca}^{2+}$ levels and studying its effect on AP-evoked release. Furthermore, sucrose solution with varying tonicity level could be applied to study, whether RRP vesicles are more responsive to suboptimal stimulation in CADPS1 ${ }^{\text {dEX2 }}$ mutant- than in control cells (Basu et al., 2007; Lipstein et al., 2017).

While changes within the fusion state of SVs may explain aspects of the synaptic phenotype of CADPS1 $1^{\mathrm{dEX} 2}$ expressing cells, the less pronounced STD in cultured CADPS1 ${ }^{\text {S399L }}$ neurons may rather be interpreted by an increase in the SV priming rate. This hypothesis is supported by the finding that tonic release, as well as the activity dependent replenishment rate of RRP vesicles were increased by the S399L mutation, while AP and sucrose triggered EPSCs, as well as the frequency of mEPSC were not changed. Whether the increase in priming rate, however, can also account for the higher PRR ratio that was detected in CADPS1 $1^{\mathrm{S} 399 \mathrm{~L}}$ cells remains unclear at the present time. This question may be addressed by future studies, in which the priming rate could be dissected in greater detail and which could further involve theoretical modeling.

Whether the changes observed in STP for these two CADPS1 mutants may have causally been involved in the biogenesis of BPD in patients, in which the mutations were initially detected, cannot conclusively be answered by the results that I obtained from cell culture experiments. Interestingly, one recent study 
observed an increase in the spontaneous AP firing rate of cultured dendate gyrus like neurons derived from induced-pluripotent stem (iPS) cells of BPD patients (Mertens et al., 2015). Whether the slightly increased mEPSC frequency observed in CADPS1 ${ }^{\mathrm{dEX} 2}$ neurons also leads to a higher AP rate was not addressed in the present study. Key insights, on whether CADPS1 ${ }^{\mathrm{dEX} 2}$ mutant proteins negatively affect behavior, or increase the firing rate of neurons in emotionally relevant circuits may come from future studies utilizing animals from the new CAPS1 ${ }^{\operatorname{dex} 2}$ mouse strain that I generated together with Fritz Benseler.

\subsection{A hypothetical model of the synaptic vesicle priming process}

Taken together, the results discussed in previous paragraphs indicate that the function of CAPSs in SV priming relies on their interaction with $\mathrm{PIP}_{2}$. Interestingly, $\mathrm{PIP}_{2}$ was found in previous studies to be the dominant inner leaflet lipid at release sites and to become metabolized to DAG at high levels of presynaptic activity (Kabachinski et al., 2014; van den Bogaart et al., 2011). In the light of findings of an essential role of the DAG-binding C1 domain of Munc13-1 in functionally compensating for a loss of CAPS proteins at high activity, it is intriguing to postulate the existence of a lipid-sensitive priming step that may precede the partial assembly of SNARE complexes. In terms of cell biology, this step may constitute a tethering process mediated either by CAPSs and $\mathrm{PIP}_{2}$ at low intraterminal $\left[\mathrm{Ca}^{2+}\right]$, or by Munc13s and DAG at high activity levels. The potential involvement of CAPSs in tethering has already been hypothesized for more than a decade (see section 4.5.2). On the other hand, the concept that Munc13s may be involved in the tethering of SVs in addition to their well-documented function in docking and SNARE complex assembly is quite novel and was published in three recent studies (Liu et al., 2016; Wickner and Rizo, 2017; Xu et al., 2017). There the authors proposed that Munc13s may bridge the distance between SVs and the plasma membrane via the functional synergy of the $\mathrm{C} 1, \mathrm{C} 2 \mathrm{~B}$ and $\mathrm{C} 2 \mathrm{C}$ domains in a lipid dependent manner (Liu et al., 2016). Following the bridging step by CAPSs and Munc13s, respectively, the MUN domain of Munc13s may orchestrate the partial assembly of SNARE complexes and thereby morphologically dock SVs (Basu et al., 2005; Imig et al., 2014; Ma et al., 2013; Wang et al., 2017; Yang et al., 2015). Accordingly, the SV priming process may consist of two sequential reactions involving a lipid-dependent tethering, or membrane bridging step and the partial assembly of trans-SNARE complexes (Fig.30).

Even though this model is attractive in terms of simplicity and since it can explain the strong phenotype observed in CAKI cells, it may be limited to the description of the priming process of a sub pool of RRP vesicles. Specifically, expression of CAPS2e fully rescued AP-evoked EPSCs, while only a small fraction of RRP vesicles was found to be primed. This may indicate that the lipid-binding $\mathrm{N}$-terminal half of CAPSs 
as well as the DAG-binding-deficient Munc13 ${ }^{\mathrm{H} 567 \mathrm{~K}}$ mutant jointly fulfill the requirements of a minimal priming machinery to sustain a basic RRP for AP-evoked release at rest. Nonetheless, the priming of other sub populations of RRP vesicles with lower release probabilities may in addition require an intact C1 domain of Munc13s as well as a functional MUN domain of CAPSs. The results presented here, do not allow to distinguish between putative roles of the MUN domain of CAPSs in promoting priming, or in stabilizing SVs and protecting these against premature fusion or depriming. However, preliminary results showing that the mEPSC frequency is increased in absence of the MUN domain of CAPS, may rather support a potential role of this domain in stabilizing the primed state of SVs (Supplementary Figure 9). Similarly, the C1 domain of Munc13 may either be required for the priming, or stabilizing of additional SVs. Lastly, the priming model proposed above may not apply to inhibitory cells. Interestingly, HFS in cultured inhibitory cells from the hippocampus of CAKI mice led to pronounced tonic EPSCs at late phases of AP trains that were comparable in their sizes to those of control neurons (Supplementary Figure 10). Furthermore, phasic transmitter release was augmented after such strong stimuli, indicating that the $\mathrm{C} 1$ domain of Munc13s is not absolutely essentially required to establish transmitter release in inhibitory CAPS deficient cells, in contrast to excitatory neurons.

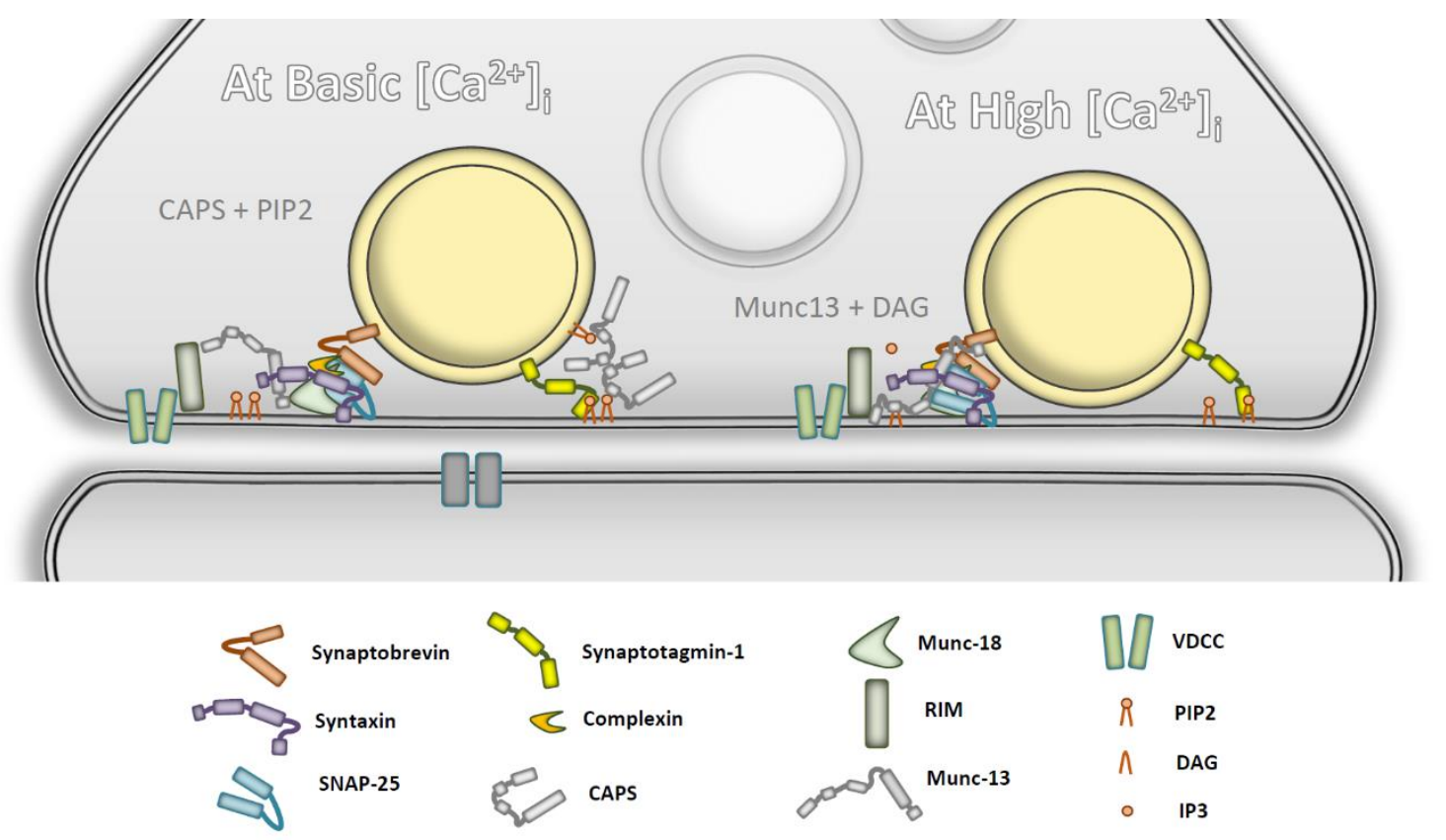

Figure 30: Hypothetical model of the SV priming process. By binding acid phospholipids on the surface of SVs and $P_{P} P_{2}$ at the plasma membrane, respectively, CAPSs dimers may tether vesicles to release sites, before partial SNARE complex assembly is orchestrated by the MUN domain of Munc13s. At high intraterminal [Ca $\left.{ }^{2+}\right]$ Munc13s may carry out the tethering step instead, by binding acidic phospholipids of SVs via their C2C domain (Liu et al., 2016) and by interacting with DAG at the plasma membrane via their C1 domain. 


\subsection{Future prospects in studying the relevance of presynaptic computation in the context of sensory processing}

Aside from the hard wired morphology of dendrites and the differential expression of ion channels, functionally dynamic synaptic connections dictate the computations by which neural circuits encode environmental stimuli (Abbott and Regehr, 2004; Kole and Stuart, 2012; London and Hausser, 2005; Stuart and Spruston, 2015; Sudhof, 2013).

Synapses perform their computations by integrating the recent history of presynaptic activity into transient adjustments of synaptic strength (Neher, 2017; Regehr, 2012; Schneggenburger et al., 2002). Such adjustments are commonly referred to as STP and have been shown to be deeply connected to the action of presynaptic proteins (de Jong and Verhage, 2009). For instance, short-term facilitation, which is thought to underlie the capacity of some synapses to low-pass filter signals, was recently shown to be regulated by the presynaptic protein synaptotagmin-7 (Jackman et al., 2016). Moreover, Munc13s are known to control the $\mathrm{Ca}^{2+}$-dependent recovery of RRP vesicles, which crucially determines the level of synaptic strength during and after HFS (Junge et al., 2004; Lipstein et al., 2013; Rhee et al., 2002; Rosenmund et al., 2002; Shin et al., 2010).

While it is generally thought that STP is crucially involved in modulating many brain functions, its functional impact on computations performed by neural circuits is still poorly understood (CastroAlamancos and Oldford, 2002; Chung et al., 2002; Mongillo et al., 2008; Reinhold et al., 2015). To address this in the future in greater detail, I conceptually and experimentally developed an approach, in which the consequences of altered synaptic strength, or STP, can directly be studied in the context of visual sensory information processing.

A number of reasons make the visual system exquisitely suitable for the systematic dissection of the contribution of STP to the processing of environmental stimuli. First, STP phenomena, such as STD have already been documented for $L 4$ neurons and were described to modulate the extent by which evoked responses adapt to repeated stimulation (Kloc and Maffei, 2014; Reinhold et al., 2015). Second, a number of theoretical models are available that postulate an important contribution of STD to shaping the response properties of $L 4$ neurons of the visual cortex (Carandini et al., 2002; Priebe and Ferster, 2012). Since different visual stimuli can be applied in an easily accessible fashion to systematically dissect different response properties, the validity of some of these models could be tested with my approach. Third and last, the visual system has been studied extensively now for many decades and much is known about its anatomical and functional organization, thereby making it a highly suitable model system for genetic manipulation approaches (Cossell et al., 2015; Harris and Mrsic-Flogel, 2013; Hubel and Wiesel, 1962; Morgan et al., 2016; Niell, 2015; Priebe and Ferster, 2012). 
In general, the experimental approach developed in this study combines the thalamic deletion of a presynaptic protein that is known to regulate synaptic strength or STP, with the electrophysiological study of sensory evoked responses in $L 4$ neurons of the visual cortex of anaesthetized mice. Preliminary results from the experimental realization of this approach are discussed below.

\subsubsection{Thalamic deletion of presynaptic proteins}

In the present study, I chose to delete CAPS1 in LGN neurons by using a previously published SERTcre driver line (Narboux-Neme et al., 2008; Zhuang et al., 2005). Yet, virtually any other component of the presynaptic release apparatus could be deleted by the same approach to manipulate differing aspects of STP in future experiments.

The cre-mediated excision of floxed alleles was confirmed in CCAPS1 1 SERTcre mice by PCR and the animals did not display any overt behavioral deficits. Results from an immunocytochemical analysis of SERTcre mice supported the previously documented expression of cre in thalamic nuclei, as well as in other brain regions (Narboux-Neme et al., 2008). Notably, one earlier study reported SERT expression also in a minor population of retinal ganglion cells (Garcia-Frigola and Herrera, 2010). However, this particular population of cells was found by the same study to only project to ipsilateral LGNs, indicating that axons crossing the midline to project to contralateral LGNs are expected to not be affected by the cre-mediated deletion. Since visual stimuli were applied in the present study to the contralateral eye and measurements were carried out in the monocular region of the visual cortex, it is unlikely that sensory processing upstream of the LGN is compromised by this experimental approach. Alternatively, future studies may make use of stereotactic injection of cre-encoding viruses into the LGN of cKO mice in order to reach a higher specificity of the genetic deletion.

\subsubsection{In-vivo patch clamp recordings allow the monitoring of sensory evoked subthreshold responses}

By monitoring subthreshold responses in layer 4 neurons using the blind in-vivo patch clamp technique, I successfully measured light evoked EPSPs in response to repetitive stimulation at $4 \mathrm{~Hz}$. My data revealed that not all of the recorded cells displayed adaptation of their responses to stimulation. This seemed to specifically apply to neurons that may have belonged to the class of $L 4$ spiny stellate cells, based on their regular firing pattern in response to depolarizing current injections (Nowak et al., 2003). Interestingly, a similar observation was made before in the barrel cortex of rats, in which subthreshold responses of layer 4 pyramidal cells were shown to adapt to whisker stimulation at $10 \mathrm{~Hz}$, while EPSPs 
in spiny stellate cells did not exhibit any depression in their amplitude (Brecht and Sakmann, 2002). Based on theoretical models that have postulated a functional significance of adaptation on various response properties, such as contrast invariance of orientation tuning, future studies may employ more complex visual stimuli to test whether adapting and non-adapting cells differ in their computations (Priebe and Ferster, 2012).

I further succeeded in obtaining a pilot recording of a putative $L 4$ neuron from the visual cortex of a mutant CAPS1 $1^{\mathrm{fl} / \mathrm{fl}}$ mouse. In this cell, subthreshold potentials were only synchronized to the first pulse of the stimulation train, while becoming desynchronized at subsequent stages. Even though it is intriguing to speculate that a pronounced deficit within the synaptic strength of thalamocortical innervation may have caused this striking phenotype, these results should be interpreted with caution, since no other data are available to support the preliminary finding. Thus, future experiments are required to test the functional consequences of the removal of CAPS1 from LGN neurons for visual processing more fully. In the framework of these studies, it could be tested whether low frequency stimulation would be associated with a facilitation of sensory evoked responses, as would be expected from previously published results, in which electrical stimulation was applied to cultured CAPS-deficient cells (Jockusch et al., 2007).

Altogether, these results strongly support the argument that this conceptually and experimentally developed approach holds much potential to successfully be applied in the future to the systematic dissection of the functional significance of STP in visual circuit function.

\section{Summary}

In this study, I found that Mover and mSYD1 are not involved in regulating synaptic transmission in cultured hippocampal neurons. Accordingly, findings from previous studies that reported a role of these proteins in modulating transmitter release, could not be supported by my results (Korber et al., 2015; Wentzel et al., 2013).

Additionally, my results indicate that the essential requirement of CAPSs in SV priming can be by-passed by a DAG-dependent priming mechanism that relies on the C1 domain of Munc13s. Interestingly, further results showed that the catalytic activity of CAPSs during priming involves their $\mathrm{PIP}_{2}$ binding $\mathrm{PH}$ domain and does not rely on their MUN domain as previously suggested by other studies. Thus, the priming process may consist of at least two sequential steps. One step is lipid dependent and requires the PH domain of CAPS at low presynaptic activity levels or the C1 domain of Munc13s at high activity. The 
second step may require the MUN domain of Munc13s and may lead to the partial assembly of SNARE complexes.

Furthermore, I functionally characterized a number of CADPS1 mutations that were found in BPD patients. For two of these mutations, I observed pronounced changes in the degree by which AP evoked EPSC amplitudes depress in response to AP trains. This finding may indicate that altered STP may be involved in the etiology of BPD.

In the last part of my PhD project, I successfully established an experimental system that may be used in the future to study STP phenomena in the context of visual information processing. 


\section{References}

Abbott, L.F., and Regehr, W.G. (2004). Synaptic computation. Nature 431, 796-803.

Ackermann, F., Waites, C.L., and Garner, C.C. (2015). Presynaptic active zones in invertebrates and vertebrates. EMBO Rep 16, 923-938.

Ahmed, S., Wittenmayer, N., Kremer, T., Hoeber, J., Kiran Akula, A., Urlaub, H., Islinger, M., Kirsch, J., Dean, C., and Dresbach, T. (2013). Mover is a homomeric phospho-protein present on synaptic vesicles. PLoS One 8, e63474.

Ann, K., Kowalchyk, J.A., Loyet, K.M., and Martin, T.F. (1997). Novel Ca2+-binding protein (CAPS) related to UNC-31 required for Ca2+-activated exocytosis. J Biol Chem 272, 19637-19640.

Ashery, U., Betz, A., Xu, T., Brose, N., and Rettig, J. (1999). An efficient method for infection of adrenal chromaffin cells using the Semliki Forest virus gene expression system. Eur I Cell Biol 78, 525-532.

Augustin, I., Betz, A., Herrmann, C., Jo, T., and Brose, N. (1999). Differential expression of two novel Munc13 proteins in rat brain. Biochem J 337 ( Pt 3), 363-371.

Avery, L., Bargmann, C.I., and Horvitz, H.R. (1993). The Caenorhabditis elegans unc-31 gene affects multiple nervous system-controlled functions. Genetics 134, 455-464.

Bai, J., Tucker, W.C., and Chapman, E.R. (2004). PIP2 increases the speed of response of synaptotagmin and steers its membrane-penetration activity toward the plasma membrane. Nat Struct Mol Biol 11, 3644.

Basu, J., Betz, A., Brose, N., and Rosenmund, C. (2007). Munc13-1 C1 domain activation lowers the energy barrier for synaptic vesicle fusion. J Neurosci 27, 1200-1210.

Basu, J., Shen, N., Dulubova, I., Lu, J., Guan, R., Guryev, O., Grishin, N.V., Rosenmund, C., and Rizo, J. (2005). A minimal domain responsible for Munc13 activity. Nat Struct Mol Biol 12, 1017-1018.

Bekkers, J.M., and Stevens, C.F. (1991). Excitatory and inhibitory autaptic currents in isolated hippocampal neurons maintained in cell culture. Proc Natl Acad Sci U S A 88, 7834-7838.

Bellocchio, E.E., Reimer, R.J., Fremeau, R.T., Jr., and Edwards, R.H. (2000). Uptake of glutamate into synaptic vesicles by an inorganic phosphate transporter. Science 289, 957-960.

Berwin, B., Floor, E., and Martin, T.F. (1998). CAPS (mammalian UNC-31) protein localizes to membranes involved in dense-core vesicle exocytosis. Neuron 21, 137-145.

Betz, A., Ashery, U., Rickmann, M., Augustin, I., Neher, E., Sudhof, T.C., Rettig, J., and Brose, N. (1998). Munc13-1 is a presynaptic phorbol ester receptor that enhances neurotransmitter release. Neuron 21, 123-136.

Betz, A., Okamoto, M., Benseler, F., and Brose, N. (1997). Direct interaction of the rat unc-13 homologue Munc13-1 with the N terminus of syntaxin. J Biol Chem 272, 2520-2526.

Betz, A., Thakur, P., Junge, H.J., Ashery, U., Rhee, J.S., Scheuss, V., Rosenmund, C., Rettig, J., and Brose, N. (2001). Functional interaction of the active zone proteins Munc13-1 and RIM1 in synaptic vesicle priming. Neuron 30, 183-196.

Blakely, R.D., and Edwards, R.H. (2012). Vesicular and plasma membrane transporters for neurotransmitters. Cold Spring Harb Perspect Biol 4.

Borst, J.G., and Soria van Hoeve, J. (2012). The calyx of Held synapse: from model synapse to auditory relay. Annu Rev Physiol 74, 199-224.

Brecht, M., and Sakmann, B. (2002). Dynamic representation of whisker deflection by synaptic potentials in spiny stellate and pyramidal cells in the barrels and septa of layer 4 rat somatosensory cortex. J Physiol 543, 49-70.

Brenner, S. (1974). The genetics of Caenorhabditis elegans. Genetics 77, 71-94.

Brose, N., Hofmann, K., Hata, Y., and Sudhof, T.C. (1995). Mammalian homologues of Caenorhabditis elegans unc-13 gene define novel family of C2-domain proteins. J Biol Chem 270, 25273-25280.

Brose, N., Rosenmund, C., and Rettig, J. (2000). Regulation of transmitter release by Unc-13 and its homologues. Curr Opin Neurobiol 10, 303-311. 
Brouns, S.J., Jore, M.M., Lundgren, M., Westra, E.R., Slijkhuis, R.J., Snijders, A.P., Dickman, M.J., Makarova, K.S., Koonin, E.V., and van der Oost, J. (2008). Small CRISPR RNAs guide antiviral defense in prokaryotes. Science 321, 960-964.

Burgalossi, A., Jung, S., Man, K.N., Nair, R., Jockusch, W.J., Wojcik, S.M., Brose, N., and Rhee, J.S. (2012). Analysis of neurotransmitter release mechanisms by photolysis of caged $\mathrm{Ca}(2)(+)$ in an autaptic neuron culture system. Nat Protoc 7, 1351-1365.

Burgalossi, A., Jung, S., Meyer, G., Jockusch, W.J., Jahn, O., Taschenberger, H., O'Connor, V.M., Nishiki, T., Takahashi, M., Brose, N., et al. (2010). SNARE protein recycling by alphaSNAP and betaSNAP supports synaptic vesicle priming. Neuron 68, 473-487.

Carandini, M., Heeger, D.J., and Senn, W. (2002). A synaptic explanation of suppression in visual cortex. J Neurosci 22, 10053-10065.

Castro-Alamancos, M.A., and Oldford, E. (2002). Cortical sensory suppression during arousal is due to the activity-dependent depression of thalamocortical synapses. J Physiol 541, 319-331.

Chen, X., Lu, J., Dulubova, I., and Rizo, J. (2008). NMR analysis of the closed conformation of syntaxin-1. J Biomol NMR 41, 43-54.

Chung, S., Li, X., and Nelson, S.B. (2002). Short-term depression at thalamocortical synapses contributes to rapid adaptation of cortical sensory responses in vivo. Neuron 34, 437-446.

Cong, L., Ran, F.A., Cox, D., Lin, S., Barretto, R., Habib, N., Hsu, P.D., Wu, X., Jiang, W., Marraffini, L.A., et al. (2013). Multiplex genome engineering using CRISPR/Cas systems. Science 339, 819-823.

Connors, B.W., and Long, M.A. (2004). Electrical synapses in the mammalian brain. Annu Rev Neurosci 27, 393-418.

Cossell, L., lacaruso, M.F., Muir, D.R., Houlton, R., Sader, E.N., Ko, H., Hofer, S.B., and Mrsic-Flogel, T.D. (2015). Functional organization of excitatory synaptic strength in primary visual cortex. Nature 518, 399403.

Crabtree, G.W., and Gogos, J.A. (2014). Synaptic plasticity, neural circuits, and the emerging role of altered short-term information processing in schizophrenia. Front Synaptic Neurosci 6, 28.

Crawford, D.C., Ramirez, D.M., Trauterman, B., Monteggia, L.M., and Kavalali, E.T. (2017). Selective molecular impairment of spontaneous neurotransmission modulates synaptic efficacy. Nat Commun 8 , 14436.

Crochet, S. (2012). Intracellular Whole-Cell Patch-Clamp Recordings of Cortical Neurons in Awake HeadRestrained Mice. T Fellin, M Halassa (Eds), Neural Network Analysis, Humana Press, New York, pp. 219235.

Crochet, S., and Petersen, C.C. (2006). Correlating whisker behavior with membrane potential in barrel cortex of awake mice. Nat Neurosci 9, 608-610.

Dai, Y., Taru, H., Deken, S.L., Grill, B., Ackley, B., Nonet, M.L., and Jin, Y. (2006). SYD-2 Liprin-alpha organizes presynaptic active zone formation through ELKS. Nat Neurosci 9, 1479-1487.

Daily, N.J., Boswell, K.L., James, D.J., and Martin, T.F. (2010). Novel interactions of CAPS (Ca2+dependent activator protein for secretion) with the three neuronal SNARE proteins required for vesicle fusion. J Biol Chem 285, 35320-35329.

de Jong, A.P., and Verhage, M. (2009). Presynaptic signal transduction pathways that modulate synaptic transmission. Curr Opin Neurobiol 19, 245-253.

Del Castillo, J., and Katz, B. (1954). Statistical factors involved in neuromuscular facilitation and depression. J Physiol 124, 574-585.

Del Castillo, J., and Katz, B. (1955). Local activity at a depolarized nerve-muscle junction. J Physiol 128, 396-411.

Delvendahl, I., Vyleta, N.P., von Gersdorff, H., and Hallermann, S. (2016). Fast, Temperature-Sensitive and Clathrin-Independent Endocytosis at Central Synapses. Neuron 90, 492-498.

Deng, L., Kaeser, P.S., Xu, W., and Sudhof, T.C. (2011). RIM proteins activate vesicle priming by reversing autoinhibitory homodimerization of Munc13. Neuron 69, 317-331.

Di Paolo, G., Moskowitz, H.S., Gipson, K., Wenk, M.R., Voronov, S., Obayashi, M., Flavell, R., Fitzsimonds, R.M., Ryan, T.A., and De Camilli, P. (2004). Impaired PtdIns(4,5)P2 synthesis in nerve terminals produces defects in synaptic vesicle trafficking. Nature 431, 415-422. 
Dulubova, I., Khvotchev, M., Liu, S., Huryeva, I., Sudhof, T.C., and Rizo, J. (2007). Munc18-1 binds directly to the neuronal SNARE complex. Proc Natl Acad Sci U S A 104, 2697-2702.

Dulubova, I., Lou, X., Lu, J., Huryeva, I., Alam, A., Schneggenburger, R., Sudhof, T.C., and Rizo, J. (2005). A Munc13/RIM/Rab3 tripartite complex: from priming to plasticity? EMBO J 24, 2839-2850.

Dulubova, I., Sugita, S., Hill, S., Hosaka, M., Fernandez, I., Sudhof, T.C., and Rizo, J. (1999). A conformational switch in syntaxin during exocytosis: role of munc18. EMBO J 18, 4372-4382.

Edwards, R.H. (2007). The neurotransmitter cycle and quantal size. Neuron 55, 835-858.

Farsi, Z., Preobraschenski, J., van den Bogaart, G., Riedel, D., Jahn, R., and Woehler, A. (2016). Singlevesicle imaging reveals different transport mechanisms between glutamatergic and GABAergic vesicles. Science 351, 981-984.

Fasshauer, D., Sutton, R.B., Brunger, A.T., and Jahn, R. (1998). Conserved structural features of the synaptic fusion complex: SNARE proteins reclassified as Q- and R-SNAREs. Proc Natl Acad Sci U S A 95, 15781-15786.

Fernandez-Busnadiego, R., Asano, S., Oprisoreanu, A.M., Sakata, E., Doengi, M., Kochovski, Z., Zurner, M., Stein, V., Schoch, S., Baumeister, W., et al. (2013). Cryo-electron tomography reveals a critical role of RIM1alpha in synaptic vesicle tethering. J Cell Biol 201, 725-740.

Fernandez-Busnadiego, R., Zuber, B., Maurer, U.E., Cyrklaff, M., Baumeister, W., and Lucic, V. (2010). Quantitative analysis of the native presynaptic cytomatrix by cryoelectron tomography. I Cell Biol 188, 145-156.

Friedrich, R., Gottfried, I., and Ashery, U. (2013). Munc13-1 Translocates to the Plasma Membrane in a Doc2B- and Calcium-Dependent Manner. Front Endocrinol (Lausanne) 4, 119.

Gage, G.J., Kipke, D.R., and Shain, W. (2012). Whole animal perfusion fixation for rodents. J Vis Exp.

Gao, Y., Zorman, S., Gundersen, G., Xi, Z., Ma, L., Sirinakis, G., Rothman, J.E., and Zhang, Y. (2012). Single reconstituted neuronal SNARE complexes zipper in three distinct stages. Science 337, 1340-1343.

Garcia-Frigola, C., and Herrera, E. (2010). Zic2 regulates the expression of Sert to modulate eye-specific refinement at the visual targets. EMBO J 29, 3170-3183.

Gerber, S.H., Rah, J.C., Min, S.W., Liu, X., de Wit, H., Dulubova, I., Meyer, A.C., Rizo, J., Arancillo, M., Hammer, R.E., et al. (2008). Conformational switch of syntaxin-1 controls synaptic vesicle fusion. Science 321, 1507-1510.

Gong, L.W., Di Paolo, G., Diaz, E., Cestra, G., Diaz, M.E., Lindau, M., De Camilli, P., and Toomre, D. (2005). Phosphatidylinositol phosphate kinase type I gamma regulates dynamics of large dense-core vesicle fusion. Proc Natl Acad Sci U S A 102, 5204-5209.

Gracheva, E.O., Burdina, A.O., Touroutine, D., Berthelot-Grosjean, M., Parekh, H., and Richmond, J.E. (2007). Tomosyn negatively regulates CAPS-dependent peptide release at Caenorhabditis elegans synapses. J Neurosci 27, 10176-10184.

Grishanin, R.N., Klenchin, V.A., Loyet, K.M., Kowalchyk, J.A., Ann, K., and Martin, T.F. (2002). Membrane association domains in $\mathrm{Ca2+-dependent} \mathrm{activator} \mathrm{protein} \mathrm{for} \mathrm{secretion} \mathrm{mediate} \mathrm{plasma} \mathrm{membrane} \mathrm{and}$ dense-core vesicle binding required for Ca2+-dependent exocytosis. J Biol Chem 277, 22025-22034.

Grishanin, R.N., Kowalchyk, J.A., Klenchin, V.A., Ann, K., Earles, C.A., Chapman, E.R., Gerona, R.R., and Martin, T.F. (2004). CAPS acts at a prefusion step in dense-core vesicle exocytosis as a PIP2 binding protein. Neuron 43, 551-562.

Gundelfinger, E.D., and Fejtova, A. (2012). Molecular organization and plasticity of the cytomatrix at the active zone. Curr Opin Neurobiol 22, 423-430.

Haider, B., and McCormick, D.A. (2009). Rapid neocortical dynamics: cellular and network mechanisms. Neuron 62, 171-189.

Hallam, S.J., Goncharov, A., McEwen, J., Baran, R., and Jin, Y. (2002). SYD-1, a presynaptic protein with PDZ, C2 and rhoGAP-like domains, specifies axon identity in C. elegans. Nat Neurosci 5, 1137-1146.

Hammarlund, M., Watanabe, S., Schuske, K., and Jorgensen, E.M. (2008). CAPS and syntaxin dock dense core vesicles to the plasma membrane in neurons. J Cell Biol 180, 483-491.

Han, G.A., Malintan, N.T., Collins, B.M., Meunier, F.A., and Sugita, S. (2010). Munc18-1 as a key regulator of neurosecretion. J Neurochem 115, 1-10. 
Han, Y., Kaeser, P.S., Sudhof, T.C., and Schneggenburger, R. (2011). RIM determines Ca(2)+ channel density and vesicle docking at the presynaptic active zone. Neuron 69, 304-316.

Hanson, P.I., Heuser, J.E., and Jahn, R. (1997a). Neurotransmitter release - four years of SNARE complexes. Curr Opin Neurobiol 7, 310-315.

Hanson, P.I., Roth, R., Morisaki, H., Jahn, R., and Heuser, J.E. (1997b). Structure and conformational changes in NSF and its membrane receptor complexes visualized by quick-freeze/deep-etch electron microscopy. Cell 90, 523-535.

Harris, K.D., and Mrsic-Flogel, T.D. (2013). Cortical connectivity and sensory coding. Nature 503, 51-58. Haucke, V., Neher, E., and Sigrist, S.J. (2011). Protein scaffolds in the coupling of synaptic exocytosis and endocytosis. Nat Rev Neurosci 12, 127-138.

Heuser, J.E., and Reese, T.S. (1973). Evidence for recycling of synaptic vesicle membrane during transmitter release at the frog neuromuscular junction. J Cell Biol 57, 315-344.

Heuser, J.E., Reese, T.S., Dennis, M.J., Jan, Y., Jan, L., and Evans, L. (1979). Synaptic vesicle exocytosis captured by quick freezing and correlated with quantal transmitter release. J Cell Biol 81, 275-300.

Honigmann, A., van den Bogaart, G., Iraheta, E., Risselada, H.J., Milovanovic, D., Mueller, V., Mullar, S., Diederichsen, U., Fasshauer, D., Grubmuller, H., et al. (2013). Phosphatidylinositol 4,5-bisphosphate clusters act as molecular beacons for vesicle recruitment. Nat Struct Mol Biol 20, 679-686.

Hosoi, N., Sakaba, T., and Neher, E. (2007). Quantitative analysis of calcium-dependent vesicle recruitment and its functional role at the calyx of Held synapse. J Neurosci 27, 14286-14298.

Hu, Z., Vashlishan-Murray, A.B., and Kaplan, J.M. (2015). NLP-12 engages different UNC-13 proteins to potentiate tonic and evoked release. J Neurosci 35, 1038-1042.

Hua, Y., Woehler, A., Kahms, M., Haucke, V., Neher, E., and Klingauf, J. (2013). Blocking endocytosis enhances short-term synaptic depression under conditions of normal availability of vesicles. Neuron 80 , 343-349.

Hubel, D.H., and Wiesel, T.N. (1962). Receptive fields, binocular interaction and functional architecture in the cat's visual cortex. J Physiol 160, 106-154.

Imig, C., Min, S.W., Krinner, S., Arancillo, M., Rosenmund, C., Sudhof, T.C., Rhee, J., Brose, N., and Cooper, B.H. (2014). The morphological and molecular nature of synaptic vesicle priming at presynaptic active zones. Neuron 84, 416-431.

Jackman, S.L., Turecek, J., Belinsky, J.E., and Regehr, W.G. (2016). The calcium sensor synaptotagmin 7 is required for synaptic facilitation. Nature 529, 88-91.

Jahn, R., and Fasshauer, D. (2012). Molecular machines governing exocytosis of synaptic vesicles. Nature 490, 201-207.

Jahn, R., and Scheller, R.H. (2006). SNAREs--engines for membrane fusion. Nat Rev Mol Cell Biol 7, 631643.

Jahne, S., Rizzoli, S.O., and Helm, M.S. (2015). The structure and function of presynaptic endosomes. Exp Cell Res 335, 172-179.

James, D.J., Khodthong, C., Kowalchyk, J.A., and Martin, T.F. (2008). Phosphatidylinositol 4,5bisphosphate regulates SNARE-dependent membrane fusion. J Cell Biol 182, 355-366.

James, D.J., Kowalchyk, J., Daily, N., Petrie, M., and Martin, T.F. (2009). CAPS drives trans-SNARE complex formation and membrane fusion through syntaxin interactions. Proc Natl Acad Sci U S A 106, 1730817313.

James, D.J., and Martin, T.F. (2013). CAPS and Munc13: CATCHRs that SNARE Vesicles. Front Endocrinol (Lausanne) 4, 187.

Jockusch, W.J., Speidel, D., Sigler, A., Sorensen, J.B., Varoqueaux, F., Rhee, J.S., and Brose, N. (2007). CAPS- 1 and CAPS-2 are essential synaptic vesicle priming proteins. Cell 131, 796-808.

Junge, H.J., Rhee, J.S., Jahn, O., Varoqueaux, F., Spiess, J., Waxham, M.N., Rosenmund, C., and Brose, N. (2004). Calmodulin and Munc13 form a Ca2+ sensor/effector complex that controls short-term synaptic plasticity. Cell 118, 389-401.

Kabachinski, G., Kielar-Grevstad, D.M., Zhang, X., James, D.J., and Martin, T.F. (2016). Resident CAPS on dense-core vesicles docks and primes vesicles for fusion. Mol Biol Cell 27, 654-668. 
Kabachinski, G., Yamaga, M., Kielar-Grevstad, D.M., Bruinsma, S., and Martin, T.F. (2014). CAPS and Munc13 utilize distinct PIP2-linked mechanisms to promote vesicle exocytosis. Mol Biol Cell 25, 508521.

Kaeser, P.S., and Regehr, W.G. (2014). Molecular mechanisms for synchronous, asynchronous, and spontaneous neurotransmitter release. Annu Rev Physiol 76, 333-363.

Kaeser, P.S., and Regehr, W.G. (2017). The readily releasable pool of synaptic vesicles. Curr Opin Neurobiol 43, 63-70.

Kandel, E.R. (2001). The molecular biology of memory storage: a dialogue between genes and synapses. Science 294, 1030-1038.

Kandel, E.R., and Spencer, W.A. (1961). Electrophysiology of hippocampal neurons. II. After-potentials and repetitive firing. J Neurophysiol 24, 243-259.

Kavalali, E.T. (2015). The mechanisms and functions of spontaneous neurotransmitter release. Nat Rev Neurosci 16, 5-16.

Khodthong, C., Kabachinski, G., James, D.J., and Martin, T.F. (2011). Munc13 homology domain-1 in CAPS/UNC31 mediates SNARE binding required for priming vesicle exocytosis. Cell Metab 14, 254-263.

Kloc, M., and Maffei, A. (2014). Target-specific properties of thalamocortical synapses onto layer 4 of mouse primary visual cortex. J Neurosci 34, 15455-15465.

Koch, H., Hofmann, K., and Brose, N. (2000). Definition of Munc13-homology-domains and characterization of a novel ubiquitously expressed Munc13 isoform. Biochem J 349, 247-253.

Kole, M.H., and Stuart, G.J. (2012). Signal processing in the axon initial segment. Neuron 73, 235-247.

Kononenko, N.L., and Haucke, V. (2015). Molecular mechanisms of presynaptic membrane retrieval and synaptic vesicle reformation. Neuron 85, 484-496.

Korber, C., Horstmann, H., Venkataramani, V., Herrmannsdorfer, F., Kremer, T., Kaiser, M., Schwenger, D.B., Ahmed, S., Dean, C., Dresbach, T., et al. (2015). Modulation of Presynaptic Release Probability by the Vertebrate-Specific Protein Mover. Neuron 87, 521-533.

Kremer, T., Kempf, C., Wittenmayer, N., Nawrotzki, R., Kuner, T., Kirsch, J., and Dresbach, T. (2007). Mover is a novel vertebrate-specific presynaptic protein with differential distribution at subsets of CNS synapses. FEBS Lett 581, 4727-4733.

Lampl, I., and Katz, Y. (2017). Neuronal adaptation in the somatosensory system of rodents. Neuroscience 343, 66-76.

Lee, J.S., Ho, W.K., Neher, E., and Lee, S.H. (2013). Superpriming of synaptic vesicles after their recruitment to the readily releasable pool. Proc Natl Acad Sci U S A 110, 15079-15084.

Li, F., Pincet, F., Perez, E., Eng, W.S., Melia, T.J., Rothman, J.E., and Tareste, D. (2007). Energetics and dynamics of SNAREpin folding across lipid bilayers. Nat Struct Mol Biol 14, 890-896.

Li, W., Ma, C., Guan, R., Xu, Y., Tomchick, D.R., and Rizo, J. (2011). The crystal structure of a Munc13 Cterminal module exhibits a remarkable similarity to vesicle tethering factors. Structure 19, 1443-1455.

Lin, R.C., and Scheller, R.H. (1997). Structural organization of the synaptic exocytosis core complex. Neuron 19, 1087-1094.

Lin, X.G., Ming, M., Chen, M.R., Niu, W.P., Zhang, Y.D., Liu, B., Jiu, Y.M., Yu, J.W., Xu, T., and Wu, Z.X. (2010). UNC-31/CAPS docks and primes dense core vesicles in C. elegans neurons. Biochem Biophys Res Commun 397, 526-531.

Lipstein, N., Sakaba, T., Cooper, B.H., Lin, K.H., Strenzke, N., Ashery, U., Rhee, J.S., Taschenberger, H., Neher, E., and Brose, N. (2013). Dynamic control of synaptic vesicle replenishment and short-term plasticity by $\mathrm{Ca}(2+)$-calmodulin-Munc13-1 signaling. Neuron 79, 82-96.

Lipstein, N., Schaks, S., Dimova, K., Kalkhof, S., Ihling, C., Kolbel, K., Ashery, U., Rhee, J., Brose, N., Sinz, A., et al. (2012). Nonconserved $\mathrm{Ca}(2+) /$ calmodulin binding sites in Munc13s differentially control synaptic short-term plasticity. Mol Cell Biol 32, 4628-4641.

Lipstein, N., Verhoeven-Duif, N.M., Michelassi, F.E., Calloway, N., van Hasselt, P.M., Pienkowska, K., van Haaften, G., van Haelst, M.M., van Empelen, R., Cuppen, I., et al. (2017). Synaptic UNC13A protein variant causes increased neurotransmission and dyskinetic movement disorder. J Clin Invest 127, 10051018. 
Liu, H., Dean, C., Arthur, C.P., Dong, M., and Chapman, E.R. (2009). Autapses and networks of hippocampal neurons exhibit distinct synaptic transmission phenotypes in the absence of synaptotagmin I. J Neurosci 29, 7395-7403.

Liu, X., Seven, A.B., Camacho, M., Esser, V., Xu, J., Trimbuch, T., Quade, B., Su, L., Ma, C., Rosenmund, C., et al. (2016). Functional synergy between the Munc13 C-terminal C1 and C2 domains. Elife 5.

London, M., and Hausser, M. (2005). Dendritic computation. Annu Rev Neurosci 28, 503-532.

Lorteije, J.A., Rusu, S.I., Kushmerick, C., and Borst, J.G. (2009). Reliability and precision of the mouse calyx of Held synapse. J Neurosci 29, 13770-13784.

Lou, X., Korogod, N., Brose, N., and Schneggenburger, R. (2008). Phorbol esters modulate spontaneous and $\mathrm{Ca} 2+-$-evoked transmitter release via acting on both Munc13 and protein kinase C. J Neurosci 28, 8257-8267.

Ma, C., Li, W., Xu, Y., and Rizo, J. (2011). Munc13 mediates the transition from the closed syntaxinMunc18 complex to the SNARE complex. Nat Struct Mol Biol 18, 542-549.

Ma, C., Su, L., Seven, A.B., Xu, Y., and Rizo, J. (2013). Reconstitution of the vital functions of Munc18 and Munc13 in neurotransmitter release. Science 339, 421-425.

Margrie, T.W., Brecht, M., and Sakmann, B. (2002). In vivo, low-resistance, whole-cell recordings from neurons in the anaesthetized and awake mammalian brain. Pflugers Arch 444, 491-498.

Marraffini, L.A. (2015). CRISPR-Cas immunity in prokaryotes. Nature 526, 55-61.

Martin, T.F. (2015). PI(4,5)P(2)-binding effector proteins for vesicle exocytosis. Biochim Biophys Acta 1851, 785-793.

Martin, T.F., and Walent, J.H. (1989). A new method for cell permeabilization reveals a cytosolic protein requirement for Ca2+-activated secretion in GH3 pituitary cells. J Biol Chem 264, 10299-10308.

Maunsell, J.H., and Gibson, J.R. (1992). Visual response latencies in striate cortex of the macaque monkey. J Neurophysiol 68, 1332-1344.

McEwen, J.M., Madison, J.M., Dybbs, M., and Kaplan, J.M. (2006). Antagonistic regulation of synaptic vesicle priming by Tomosyn and UNC-13. Neuron 51, 303-315.

Mertens, J., Wang, Q.W., Kim, Y., Yu, D.X., Pham, S., Yang, B., Zheng, Y., Diffenderfer, K.E., Zhang, J., Soltani, S., et al. (2015). Differential responses to lithium in hyperexcitable neurons from patients with bipolar disorder. Nature 527, 95-99.

Miller, K.G., Alfonso, A., Nguyen, M., Crowell, J.A., Johnson, C.D., and Rand, J.B. (1996). A genetic selection for Caenorhabditis elegans synaptic transmission mutants. Proc Natl Acad Sci U S A 93, 1259312598.

Milosevic, I., Sorensen, J.B., Lang, T., Krauss, M., Nagy, G., Haucke, V., Jahn, R., and Neher, E. (2005). Plasmalemmal phosphatidylinositol-4,5-bisphosphate level regulates the releasable vesicle pool size in chromaffin cells. J Neurosci 25, 2557-2565.

Misura, K.M., Scheller, R.H., and Weis, W.I. (2000). Three-dimensional structure of the neuronal-Sec1syntaxin 1a complex. Nature 404, 355-362.

Mongillo, G., Barak, O., and Tsodyks, M. (2008). Synaptic theory of working memory. Science 319, 15431546.

Morgan, J.L., Berger, D.R., Wetzel, A.W., and Lichtman, J.W. (2016). The Fuzzy Logic of Network Connectivity in Mouse Visual Thalamus. Cell 165, 192-206.

Murthy, V.N., Schikorski, T., Stevens, C.F., and Zhu, Y. (2001). Inactivity produces increases in neurotransmitter release and synapse size. Neuron 32, 673-682.

Nair, R., Lauks, J., Jung, S., Cooke, N.E., de Wit, H., Brose, N., Kilimann, M.W., Verhage, M., and Rhee, J. (2013). Neurobeachin regulates neurotransmitter receptor trafficking to synapses. J Cell Biol 200, 6180.

Narayanan, R.T., Mohan, H., Broersen, R., de Haan, R., Pieneman, A.W., and de Kock, C.P. (2014). Juxtasomal biocytin labeling to study the structure-function relationship of individual cortical neurons. $\mathrm{J}$ Vis Exp, e51359.

Narboux-Neme, N., Evrard, A., Ferezou, I., Erzurumlu, R.S., Kaeser, P.S., Laine, J., Rossier, J., Ropert, N., Sudhof, T.C., and Gaspar, P. (2012). Neurotransmitter release at the thalamocortical synapse instructs barrel formation but not axon patterning in the somatosensory cortex. J Neurosci 32, 6183-6196. 
Narboux-Neme, N., Pavone, L.M., Avallone, L., Zhuang, X., and Gaspar, P. (2008). Serotonin transporter transgenic (SERTcre) mouse line reveals developmental targets of serotonin specific reuptake inhibitors (SSRIs). Neuropharmacology 55, 994-1005.

Neher, E. (2010). What is Rate-Limiting during Sustained Synaptic Activity: Vesicle Supply or the Availability of Release Sites. Front Synaptic Neurosci 2, 144.

Neher, E. (2015). Merits and Limitations of Vesicle Pool Models in View of Heterogeneous Populations of Synaptic Vesicles. Neuron 87, 1131-1142.

Neher, E. (2017). Some Subtle Lessons from the Calyx of Held Synapse. Biophys J 112, 215-223.

Neher, E., and Sakaba, T. (2008). Multiple roles of calcium ions in the regulation of neurotransmitter release. Neuron 59, 861-872.

Nguyen Truong, C.Q., Nestvogel, D., Ratai, O., Schirra, C., Stevens, D.R., Brose, N., Rhee, J., and Rettig, J. (2014). Secretory vesicle priming by CAPS is independent of its SNARE-binding MUN domain. Cell Rep 9, 902-909.

Niell, C.M. (2015). Cell types, circuits, and receptive fields in the mouse visual cortex. Annu Rev Neurosci 38, 413-431.

Nowak, L.G., Azouz, R., Sanchez-Vives, M.V., Gray, C.M., and McCormick, D.A. (2003). Electrophysiological classes of cat primary visual cortical neurons in vivo as revealed by quantitative analyses. J Neurophysiol 89, 1541-1566.

Oelkers, M., Witt, H., Halder, P., Jahn, R., and Janshoff, A. (2016). SNARE-mediated membrane fusion trajectories derived from force-clamp experiments. Proc Natl Acad Sci U S A 113, 13051-13056.

Orban, P.C., Chui, D., and Marth, J.D. (1992). Tissue- and site-specific DNA recombination in transgenic mice. Proc Natl Acad Sci U S A 89, 6861-6865.

Owald, D., Fouquet, W., Schmidt, M., Wichmann, C., Mertel, S., Depner, H., Christiansen, F., Zube, C., Quentin, C., Korner, J., et al. (2010). A Syd-1 homologue regulates pre- and postsynaptic maturation in Drosophila. J Cell Biol 188, 565-579.

Park, Y., Seo, J.B., Fraind, A., Perez-Lara, A., Yavuz, H., Han, K., Jung, S.R., Kattan, I., Walla, P.J., Choi, M., et al. (2015). Synaptotagmin-1 binds to PIP(2)-containing membrane but not to SNAREs at physiological ionic strength. Nat Struct Mol Biol 22, 815-823.

Parsaud, L., Li, L., Jung, C.H., Park, S., Saw, N.M., Park, S., Kim, M.Y., and Sugita, S. (2013). Calciumdependent activator protein for secretion 1 (CAPS1) binds to syntaxin-1 in a distinct mode from Munc131. J Biol Chem 288, 23050-23063.

Patel, M.R., Lehrman, E.K., Poon, V.Y., Crump, J.G., Zhen, M., Bargmann, C.I., and Shen, K. (2006). Hierarchical assembly of presynaptic components in defined C. elegans synapses. Nat Neurosci 9, 14881498.

Pei, J., Ma, C., Rizo, J., and Grishin, N.V. (2009). Remote homology between Munc13 MUN domain and vesicle tethering complexes. J Mol Biol 391, 509-517.

Petrie, M., Esquibel, J., Kabachinski, G., Maciuba, S., Takahashi, H., Edwardson, J.M., and Martin, T.F. (2016). The Vesicle Priming Factor CAPS Functions as a Homodimer via C2 Domain Interactions to Promote Regulated Vesicle Exocytosis. J Biol Chem 291, 21257-21270.

Priebe, N.J., and Ferster, D. (2012). Mechanisms of neuronal computation in mammalian visual cortex. Neuron 75, 194-208.

Ran, F.A., Hsu, P.D., Wright, J., Agarwala, V., Scott, D.A., and Zhang, F. (2013). Genome engineering using the CRISPR-Cas9 system. Nat Protoc 8, 2281-2308.

Regehr, W.G. (2012). Short-term presynaptic plasticity. Cold Spring Harb Perspect Biol 4, a005702.

Reinhold, K., Lien, A.D., and Scanziani, M. (2015). Distinct recurrent versus afferent dynamics in cortical visual processing. Nat Neurosci 18, 1789-1797.

Renden, R., Berwin, B., Davis, W., Ann, K., Chin, C.T., Kreber, R., Ganetzky, B., Martin, T.F., and Broadie, K. (2001). Drosophila CAPS is an essential gene that regulates dense-core vesicle release and synaptic vesicle fusion. Neuron 31, 421-437.

Rhee, J.S., Betz, A., Pyott, S., Reim, K., Varoqueaux, F., Augustin, I., Hesse, D., Sudhof, T.C., Takahashi, M., Rosenmund, C., et al. (2002). Beta phorbol ester- and diacylglycerol-induced augmentation of transmitter release is mediated by Munc13s and not by PKCs. Cell 108, 121-133. 
Richmond, J.E., Davis, W.S., and Jorgensen, E.M. (1999). UNC-13 is required for synaptic vesicle fusion in C. elegans. Nat Neurosci 2, 959-964.

Richmond, J.E., Weimer, R.M., and Jorgensen, E.M. (2001). An open form of syntaxin bypasses the requirement for UNC-13 in vesicle priming. Nature 412, 338-341.

Rickman, C., Medine, C.N., Bergmann, A., and Duncan, R.R. (2007). Functionally and spatially distinct modes of munc18-syntaxin 1 interaction. J Biol Chem 282, 12097-12103.

Ripamonti, S., Ambrozkiewicz, M.C., Guzzi, F., Gravati, M., Biella, G., Bormuth, I., Hammer, M., Tuffy, L.P., Sigler, A., Kawabe, H., et al. (2017). Transient oxytocin signaling primes the development and function of excitatory hippocampal neurons. Elife 6.

Rizo, J., and Sudhof, T.C. (2012). The membrane fusion enigma: SNAREs, Sec1/Munc18 proteins, and their accomplices--guilty as charged? Annu Rev Cell Dev Biol 28, 279-308.

Rizo, J., and Xu, J. (2015). The Synaptic Vesicle Release Machinery. Annu Rev Biophys 44, 339-367.

Rizzoli, S.O. (2014). Synaptic vesicle recycling: steps and principles. EMBO J 33, 788-822.

Rodriguez-Castaneda, F., Maestre-Martinez, M., Coudevylle, N., Dimova, K., Junge, H., Lipstein, N., Lee, D., Becker, S., Brose, N., Jahn, O., et al. (2010). Modular architecture of Munc13/calmodulin complexes: dual regulation by Ca2+ and possible function in short-term synaptic plasticity. EMBO J 29, 680-691.

Rosenmund, C., Sigler, A., Augustin, I., Reim, K., Brose, N., and Rhee, J.S. (2002). Differential control of vesicle priming and short-term plasticity by Munc13 isoforms. Neuron 33, 411-424.

Rosenmund, C., and Stevens, C.F. (1996). Definition of the readily releasable pool of vesicles at hippocampal synapses. Neuron 16, 1197-1207.

Sadakata, T., Itakura, M., Kozaki, S., Sekine, Y., Takahashi, M., and Furuichi, T. (2006). Differential distributions of the $\mathrm{Ca} 2+$-dependent activator protein for secretion family proteins (CAPS2 and CAPS1) in the mouse brain. J Comp Neurol 495, 735-753.

Sadakata, T., Shinoda, Y., Oka, M., Sekine, Y., Sato, Y., Saruta, C., Miwa, H., Tanaka, M., Itohara, S., and Furuichi, T. (2012). Reduced axonal localization of a Caps2 splice variant impairs axonal release of BDNF and causes autistic-like behavior in mice. Proc Natl Acad Sci U S A 109, 21104-21109.

Sadakata, T., Shinoda, Y., Sato, A., Iguchi, H., Ishii, C., Matsuo, M., Yamaga, R., and Furuichi, T. (2013). Mouse models of mutations and variations in autism spectrum disorder-associated genes: mice expressing Caps2/Cadps2 copy number and alternative splicing variants. Int J Environ Res Public Health 10, 6335-6353.

Sadakata, T., Washida, M., and Furuichi, T. (2007a). Alternative splicing variations in mouse CAPS2: differential expression and functional properties of splicing variants. BMC Neurosci 8, 25.

Sadakata, T., Washida, M., Iwayama, Y., Shoji, S., Sato, Y., Ohkura, T., Katoh-Semba, R., Nakajima, M., Sekine, Y., Tanaka, M., et al. (2007b). Autistic-like phenotypes in Cadps2-knockout mice and aberrant CADPS2 splicing in autistic patients. J Clin Invest 117, 931-943.

Sassa, T., Harada, S., Ogawa, H., Rand, J.B., Maruyama, I.N., and Hosono, R. (1999). Regulation of the UNC-18-Caenorhabditis elegans syntaxin complex by UNC-13. J Neurosci 19, 4772-4777.

Scheuss, V., Schneggenburger, R., and Neher, E. (2002). Separation of presynaptic and postsynaptic contributions to depression by covariance analysis of successive EPSCs at the calyx of Held synapse. J Neurosci 22, 728-739.

Schiavo, G., Gu, Q.M., Prestwich, G.D., Sollner, T.H., and Rothman, J.E. (1996). Calcium-dependent switching of the specificity of phosphoinositide binding to synaptotagmin. Proc Natl Acad Sci U S A 93, 13327-13332.

Schikorski, T., and Stevens, C.F. (1997). Quantitative ultrastructural analysis of hippocampal excitatory synapses. J Neurosci 17, 5858-5867.

Schikorski, T., and Stevens, C.F. (2001). Morphological correlates of functionally defined synaptic vesicle populations. Nat Neurosci 4, 391-395.

Schneggenburger, R., and Forsythe, I.D. (2006). The calyx of Held. Cell Tissue Res 326, 311-337.

Schneggenburger, R., Sakaba, T., and Neher, E. (2002). Vesicle pools and short-term synaptic depression: lessons from a large synapse. Trends Neurosci 25, 206-212. 
Schoch, S., Castillo, P.E., Jo, T., Mukherjee, K., Geppert, M., Wang, Y., Schmitz, F., Malenka, R.C., and Sudhof, T.C. (2002). RIM1alpha forms a protein scaffold for regulating neurotransmitter release at the active zone. Nature 415, 321-326.

Schoch, S., Mittelstaedt, T., Kaeser, P.S., Padgett, D., Feldmann, N., Chevaleyre, V., Castillo, P.E., Hammer, R.E., Han, W., Schmitz, F., et al. (2006). Redundant functions of RIM1alpha and RIM2alpha in $\mathrm{Ca}(2+)$-triggered neurotransmitter release. EMBO J 25, 5852-5863.

Shen, N., Guryev, O., and Rizo, J. (2005). Intramolecular occlusion of the diacylglycerol-binding site in the C1 domain of munc13-1. Biochemistry 44, 1089-1096.

Shin, O.H., Lu, J., Rhee, J.S., Tomchick, D.R., Pang, Z.P., Wojcik, S.M., Camacho-Perez, M., Brose, N., Machius, M., Rizo, J., et al. (2010). Munc13 C2B domain is an activity-dependent Ca2+ regulator of synaptic exocytosis. Nat Struct Mol Biol 17, 280-288.

Shiratsuchi, T., Oda, K., Nishimori, H., Suzuki, M., Takahashi, E., Tokino, T., and Nakamura, Y. (1998). Cloning and characterization of BAP3 (BAl-associated protein 3), a C2 domain-containing protein that interacts with BAl1. Biochem Biophys Res Commun 251, 158-165.

Siksou, L., Varoqueaux, F., Pascual, O., Triller, A., Brose, N., and Marty, S. (2009). A common molecular basis for membrane docking and functional priming of synaptic vesicles. Eur J Neurosci 30, 49-56.

Sollner, T., Whiteheart, S.W., Brunner, M., Erdjument-Bromage, H., Geromanos, S., Tempst, P., and Rothman, J.E. (1993). SNAP receptors implicated in vesicle targeting and fusion. Nature 362, 318-324.

Sorensen, J.B., Wiederhold, K., Muller, E.M., Milosevic, I., Nagy, G., de Groot, B.L., Grubmuller, H., and Fasshauer, D. (2006). Sequential N- to C-terminal SNARE complex assembly drives priming and fusion of secretory vesicles. EMBO J 25, 955-966.

Soykan, T., Kaempf, N., Sakaba, T., Vollweiter, D., Goerdeler, F., Puchkov, D., Kononenko, N.L., and Haucke, V. (2017). Synaptic Vesicle Endocytosis Occurs on Multiple Timescales and Is Mediated by Formin-Dependent Actin Assembly. Neuron 93, 854-866 e854.

Soykan, T., Maritzen, T., and Haucke, V. (2016). Modes and mechanisms of synaptic vesicle recycling. Curr Opin Neurobiol 39, 17-23.

Speese, S., Petrie, M., Schuske, K., Ailion, M., Ann, K., Iwasaki, K., Jorgensen, E.M., and Martin, T.F. (2007). UNC-31 (CAPS) is required for dense-core vesicle but not synaptic vesicle exocytosis in Caenorhabditis elegans. J Neurosci 27, 6150-6162.

Speidel, D., Varoqueaux, F., Enk, C., Nojiri, M., Grishanin, R.N., Martin, T.F., Hofmann, K., Brose, N., and Reim, K. (2003). A family of Ca2+-dependent activator proteins for secretion: comparative analysis of structure, expression, localization, and function. J Biol Chem 278, 52802-52809.

Stevens, C.F., and Sullivan, J.M. (1998). Regulation of the readily releasable vesicle pool by protein kinase C. Neuron 21, 885-893.

Stevens, D.R., and Rettig, J. (2009). The Ca(2+)-dependent activator protein for secretion CAPS: do I dock or do I prime? Mol Neurobiol 39, 62-72.

Stuart, G.J., and Spruston, N. (2015). Dendritic integration: 60 years of progress. Nat Neurosci 18, 17131721.

Sudhof, T.C. (2004). The synaptic vesicle cycle. Annu Rev Neurosci 27, 509-547.

Sudhof, T.C. (2012a). Calcium control of neurotransmitter release. Cold Spring Harb Perspect Biol 4, a011353.

Sudhof, T.C. (2012b). The presynaptic active zone. Neuron 75, 11-25.

Sudhof, T.C. (2013). Neurotransmitter release: the last millisecond in the life of a synaptic vesicle. Neuron 80, 675-690.

Sudhof, T.C., and Rothman, J.E. (2009). Membrane fusion: grappling with SNARE and SM proteins. Science 323, 474-477.

Sutton, R.B., Fasshauer, D., Jahn, R., and Brunger, A.T. (1998). Crystal structure of a SNARE complex involved in synaptic exocytosis at 2.4 A resolution. Nature 395, 347-353.

Takamori, S., Rhee, J.S., Rosenmund, C., and Jahn, R. (2000). Identification of a vesicular glutamate transporter that defines a glutamatergic phenotype in neurons. Nature 407, 189-194.

Tandon, A., Bannykh, S., Kowalchyk, J.A., Banerjee, A., Martin, T.F., and Balch, W.E. (1998). Differential regulation of exocytosis by calcium and CAPS in semi-intact synaptosomes. Neuron 21, 147-154. 
Taschenberger, H., Woehler, A., and Neher, E. (2016). Superpriming of synaptic vesicles as a common basis for intersynapse variability and modulation of synaptic strength. Proc Natl Acad Sci U S A 113, E4548-4557.

Thanawala, M.S., and Regehr, W.G. (2013). Presynaptic calcium influx controls neurotransmitter release in part by regulating the effective size of the readily releasable pool. J Neurosci 33, 4625-4633.

Toonen, R.F., Wierda, K., Sons, M.S., de Wit, H., Cornelisse, L.N., Brussaard, A., Plomp, J.J., and Verhage, M. (2006). Munc18-1 expression levels control synapse recovery by regulating readily releasable pool size. Proc Natl Acad Sci U S A 103, 18332-18337.

Trimbuch, T., and Rosenmund, C. (2016). Should I stop or should I go? The role of complexin in neurotransmitter release. Nat Rev Neurosci 17, 118-125.

Truckenbrodt, S., and Rizzoli, S.O. (2014). Spontaneous vesicle recycling in the synaptic bouton. Front Cell Neurosci 8, 409.

Tsien, J.Z. (2016). Cre-Lox Neurogenetics: 20 Years of Versatile Applications in Brain Research and Counting. Front Genet 7, 19.

Tsien, J.Z., Chen, D.F., Gerber, D., Tom, C., Mercer, E.H., Anderson, D.J., Mayford, M., Kandel, E.R., and Tonegawa, S. (1996). Subregion- and cell type-restricted gene knockout in mouse brain. Cell 87, 13171326.

Urnavicius, L., Zhang, K., Diamant, A.G., Motz, C., Schlager, M.A., Yu, M., Patel, N.A., Robinson, C.V., and Carter, A.P. (2015). The structure of the dynactin complex and its interaction with dynein. Science 347, 1441-1446.

van den Bogaart, G., Meyenberg, K., Risselada, H.J., Amin, H., Willig, K.I., Hubrich, B.E., Dier, M., Hell, S.W., Grubmuller, H., Diederichsen, U., et al. (2011). Membrane protein sequestering by ionic proteinlipid interactions. Nature 479, 552-555.

Varoqueaux, F., Sigler, A., Rhee, J.S., Brose, N., Enk, C., Reim, K., and Rosenmund, C. (2002). Total arrest of spontaneous and evoked synaptic transmission but normal synaptogenesis in the absence of Munc13-mediated vesicle priming. Proc Natl Acad Sci U S A 99, 9037-9042.

Verhage, M., Maia, A.S., Plomp, J.J., Brussaard, A.B., Heeroma, J.H., Vermeer, H., Toonen, R.F., Hammer, R.E., van den Berg, T.K., Missler, M., et al. (2000). Synaptic assembly of the brain in the absence of neurotransmitter secretion. Science 287, 864-869.

von Gersdorff, H., and Borst, J.G. (2002). Short-term plasticity at the calyx of Held. Nat Rev Neurosci 3, 53-64.

Walent, J.H., Porter, B.W., and Martin, T.F. (1992). A novel 145 kd brain cytosolic protein reconstitutes $\mathrm{Ca}(2+)$-regulated secretion in permeable neuroendocrine cells. Cell 70, 765-775.

Walter, A.M., Wiederhold, K., Bruns, D., Fasshauer, D., and Sorensen, J.B. (2010). Synaptobrevin Nterminally bound to syntaxin-SNAP-25 defines the primed vesicle state in regulated exocytosis. J Cell Biol 188, 401-413.

Wang, H., Yang, H., Shivalila, C.S., Dawlaty, M.M., Cheng, A.W., Zhang, F., and Jaenisch, R. (2013). Onestep generation of mice carrying mutations in multiple genes by CRISPR/Cas-mediated genome engineering. Cell 153, 910-918.

Wang, S., Choi, U.B., Gong, J., Yang, X., Li, Y., Wang, A.L., Yang, X., Brunger, A.T., and Ma, C. (2017). Conformational change of syntaxin linker region induced by Munc13s initiates SNARE complex formation in synaptic exocytosis. EMBO J 36, 816-829.

Wang, S.S., Held, R.G., Wong, M.Y., Liu, C., Karakhanyan, A., and Kaeser, P.S. (2016). Fusion Competent Synaptic Vesicles Persist upon Active Zone Disruption and Loss of Vesicle Docking. Neuron 91, 777-791. Watanabe, S., Liu, Q., Davis, M.W., Hollopeter, G., Thomas, N., Jorgensen, N.B., and Jorgensen, E.M. (2013a). Ultrafast endocytosis at Caenorhabditis elegans neuromuscular junctions. Elife 2, e00723.

Watanabe, S., Rost, B.R., Camacho-Perez, M., Davis, M.W., Sohl-Kielczynski, B., Rosenmund, C., and Jorgensen, E.M. (2013b). Ultrafast endocytosis at mouse hippocampal synapses. Nature 504, 242-247. Watanabe, S., Trimbuch, T., Camacho-Perez, M., Rost, B.R., Brokowski, B., Sohl-Kielczynski, B., Felies, A., Davis, M.W., Rosenmund, C., and Jorgensen, E.M. (2014). Clathrin regenerates synaptic vesicles from endosomes. Nature 515, 228-233. 
Wentzel, C., Sommer, J.E., Nair, R., Stiefvater, A., Sibarita, J.B., and Scheiffele, P. (2013). mSYD1A, a mammalian synapse-defective-1 protein, regulates synaptogenic signaling and vesicle docking. Neuron 78, 1012-1023.

Wickner, W., and Rizo, J. (2017). A cascade of multiple proteins and lipids catalyzes membrane fusion. Mol Biol Cell 28, 707-711.

Wojcik, S.M., and Brose, N. (2007). Regulation of membrane fusion in synaptic excitation-secretion coupling: speed and accuracy matter. Neuron 55, 11-24.

Xu, J., Camacho, M., Xu, Y., Esser, V., Liu, X., Trimbuch, T., Pan, Y.Z., Ma, C., Tomchick, D.R., Rosenmund, C., et al. (2017). Mechanistic insights into neurotransmitter release and presynaptic plasticity from the crystal structure of Munc13-1 C1C2BMUN. Elife 6.

Yang, X., Cao, P., and Sudhof, T.C. (2013). Deconstructing complexin function in activating and clamping Ca2+-triggered exocytosis by comparing knockout and knockdown phenotypes. Proc Natl Acad Sci U S A 110, 20777-20782.

Yang, X., Wang, S., Sheng, Y., Zhang, M., Zou, W., Wu, L., Kang, L., Rizo, J., Zhang, R., Xu, T., et al. (2015). Syntaxin opening by the MUN domain underlies the function of Munc13 in synaptic-vesicle priming. Nat Struct Mol Biol 22, 547-554.

Yu, I.M., and Hughson, F.M. (2010). Tethering factors as organizers of intracellular vesicular traffic. Annu Rev Cell Dev Biol 26, 137-156.

Zhao, M., Wu, S., Zhou, Q., Vivona, S., Cipriano, D.J., Cheng, Y., and Brunger, A.T. (2015). Mechanistic insights into the recycling machine of the SNARE complex. Nature 518, 61-67.

Zhou, K.M., Dong, Y.M., Ge, Q., Zhu, D., Zhou, W., Lin, X.G., Liang, T., Wu, Z.X., and Xu, T. (2007). PKA activation bypasses the requirement for UNC-31 in the docking of dense core vesicles from $C$. elegans neurons. Neuron 56, 657-669.

Zhou, P., Pang, Z.P., Yang, X., Zhang, Y., Rosenmund, C., Bacaj, T., and Sudhof, T.C. (2013). Syntaxin-1 Npeptide and Habc-domain perform distinct essential functions in synaptic vesicle fusion. EMBO J 32, 159-171.

Zhuang, X., Masson, J., Gingrich, J.A., Rayport, S., and Hen, R. (2005). Targeted gene expression in dopamine and serotonin neurons of the mouse brain. J Neurosci Methods 143, $27-32$. 


\section{Supplementary}

A

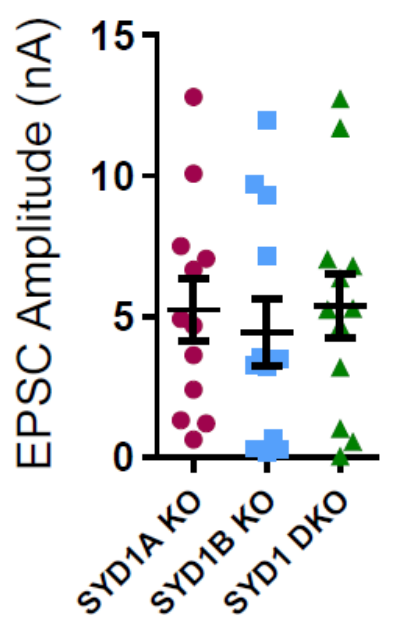

$\mathrm{D}$

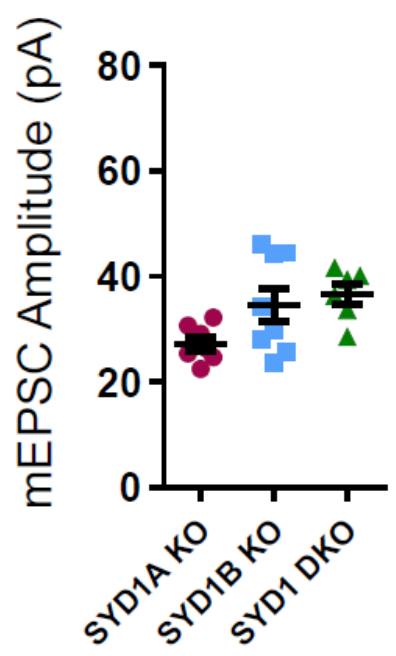

B

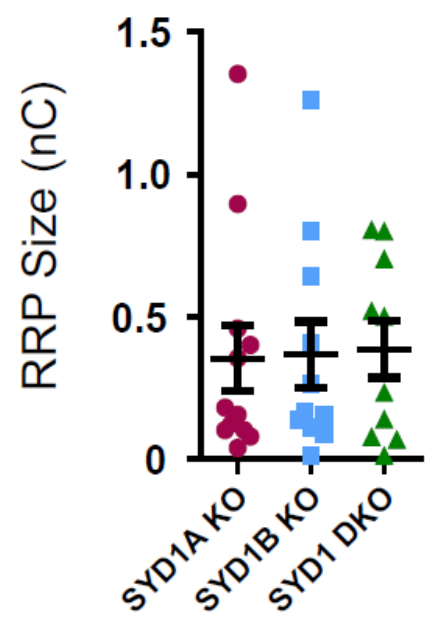

$\mathrm{E}$

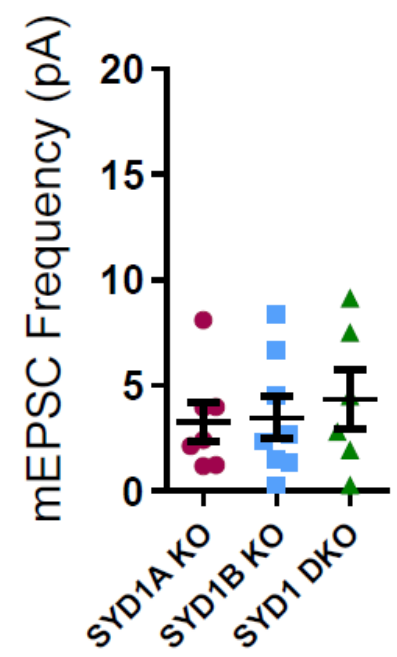

C

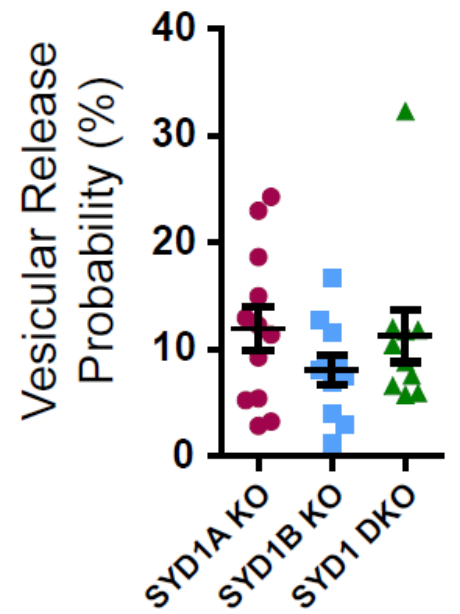

$\mathrm{F}$

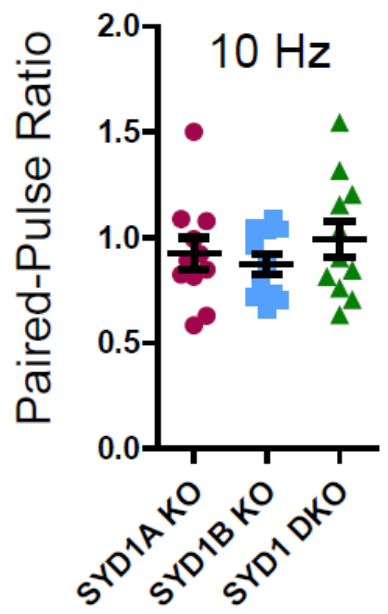

Supplementary Figure 1: Synaptic transmission is not altered in cultured neurons from mSYD1B or mSYD1-A,B DKO mice, respectively. (A) Mean EPSC amplitude of cultured neurons with indicated genotypes ( $n=12$ cells each). (B) RRP size and (C) Vesicular release probability $(n=12,11$ and 10 cells). (D) and (E) Mean amplitude and frequency of $\mathrm{mEPSCS}(n=7,8$ and 6 cells). (F) Paired-pulse ratio between two consecutive EPSCs at a time interval of $100 \mathrm{~ms}$ $(n=11$ cells each). (Bars in plots depict mean and SEM. $N=1$ culture). 


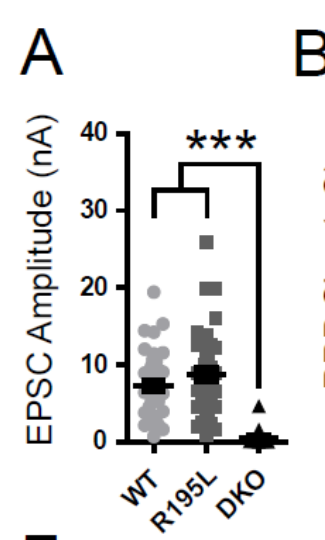

$\mathrm{F}$

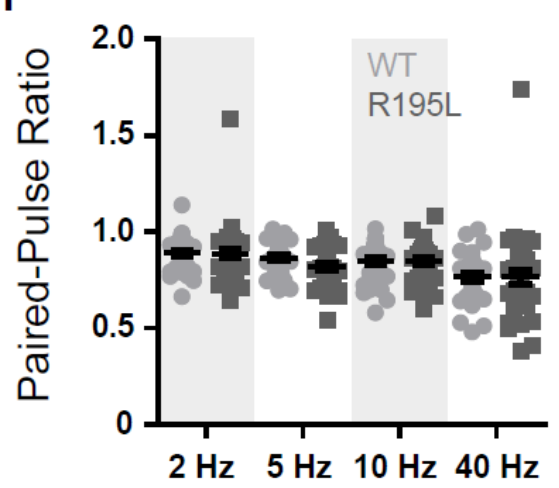

C

D

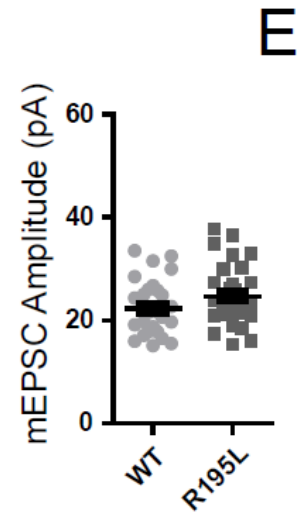

$\mathrm{E}$
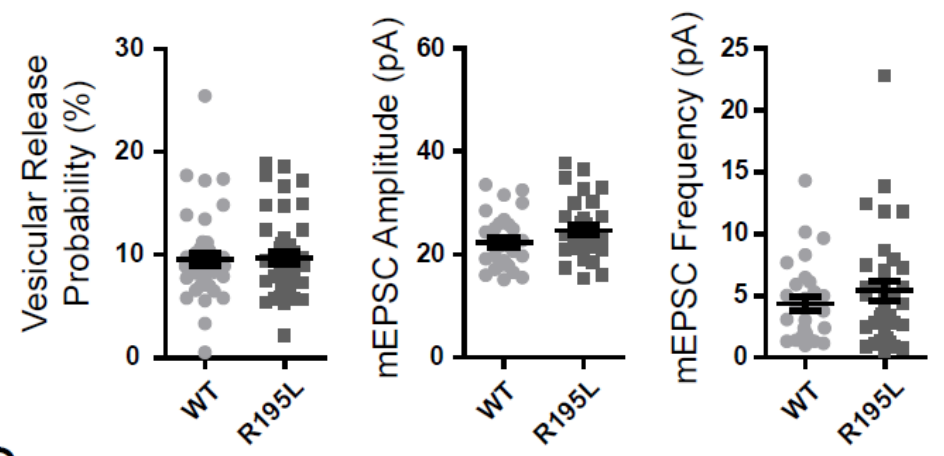

G

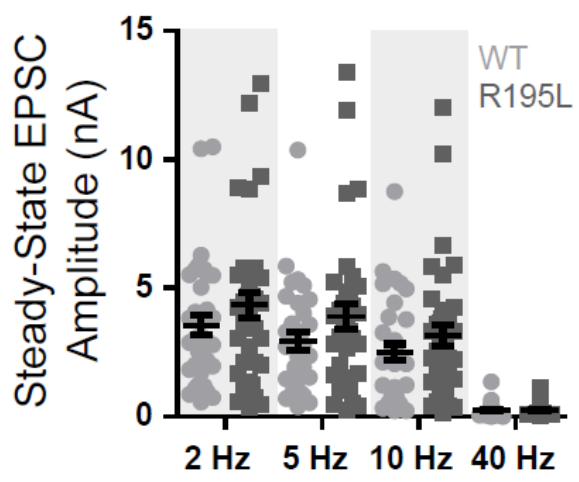

Supplementary Figure 2: Synaptic transmission is fully rescued in cultured CAPS DKO neurons expressing CADPS- ${ }^{R 195 L}$. (A) Mean EPSC response triggered by single APS ( $n=43,43$ and 28 cells, of which 4 DKO cells showed no AP-evoked responses, Kruskal-Wallis test, Dunn's Multiple Comparison Test, $\left.{ }^{* * * P}<0.001\right)$. (B) RRP size determined by measuring the charge of sucrose-evoked responses ( $n=43$ cells each). (C) Mean vesicular release probability calculated by dividing the charge of AP-evoked EPSCS by the charge of sucrose evoked responses ( $n=43$ cells each). (D) and (E) Amplitude and frequency of mEPSCS measured during TTX application ( $n=31$ and 34 cells). (F) Pairedpulse ratio during AP trains at the indicated frequencies ( $n=32-38$ cells). (J) Averaged steady-state EPSC responses during trains of APs at indicated frequencies ( $n=32-38$ cells). (Bars in plots depict mean and SEM. $N=6$ cultures). 


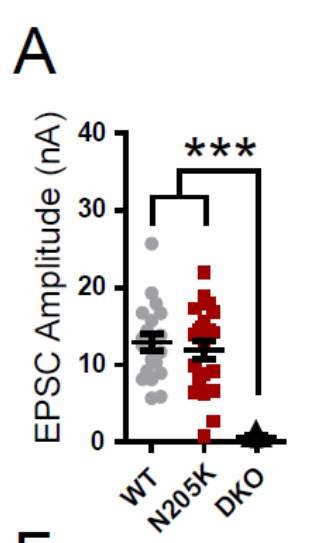

$\mathrm{F}$

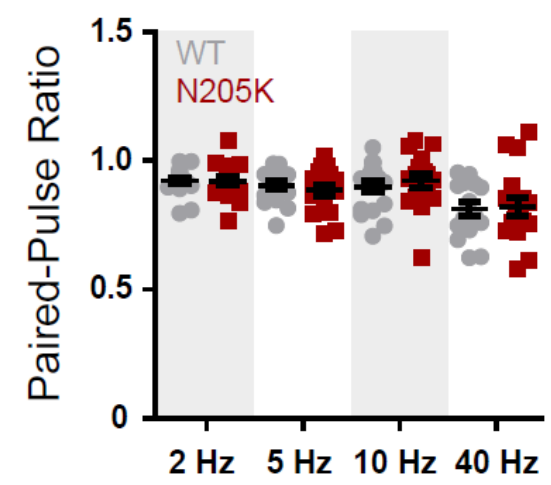

C

D

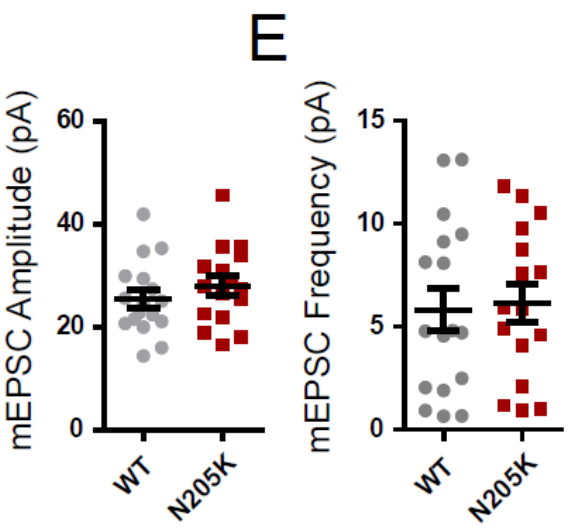

G

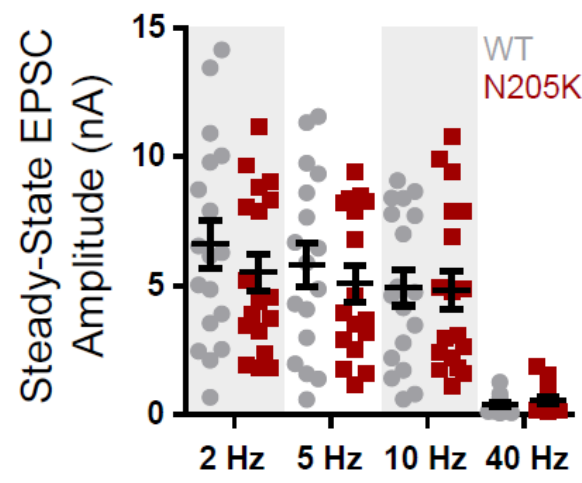

Supplementary Figure 3: Synaptic transmission is fully rescued in cultured CAPS DKO neurons expressing CADPS- ${ }^{\text {N205K }}$. (A) Mean amplitude of AP-triggered EPSCS $(n=20,21$ and 12 cells, of which 1 DKO cell showed no AP-evoked responses, Kruskal-Wallis test, Dunn's Multiple Comparison Test, $\left.{ }^{* * *} P<0.001\right)$. (B) RRP size determined by sucrose solution application ( $n=19$ and 21 cells). (C) Mean vesicular release probability calculated by dividing the charge of AP-evoked EPSCs by the charge of sucrose evoked responses ( $n=19$ and 21 cells). (D) and (E) Amplitude and frequency of spontaneously occurring mEPSCS ( $n=17$ and 16 cells). (F) Paired-pulse ratio during AP trains at the indicated frequencies ( $n=17-18$ cells). (J) Averaged steady-state EPSC responses during trains of APs at indicated frequencies ( $n=17-18$ cells). (Bars in plots depict mean and SEM. $N=3$ cultures). 


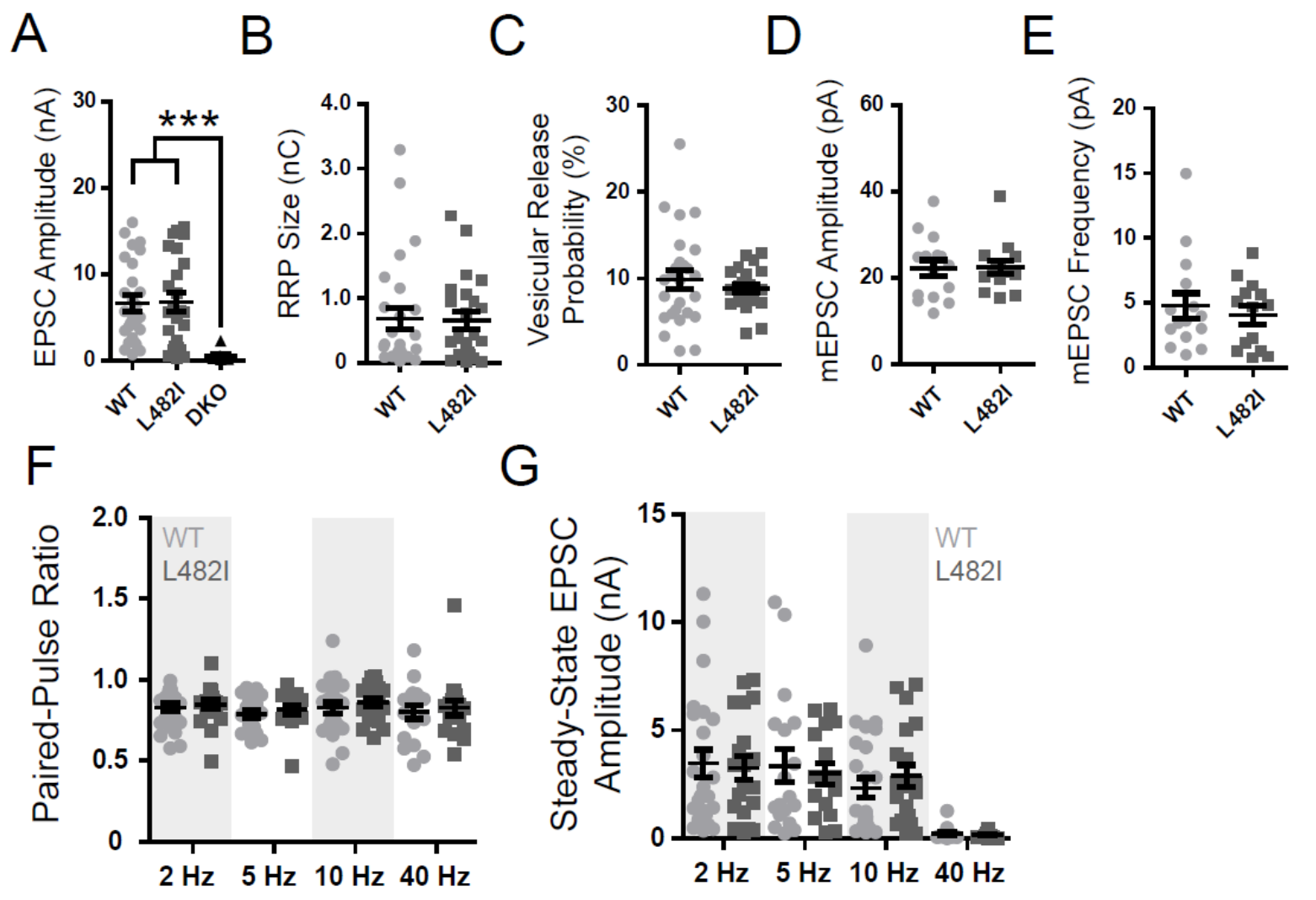

Supplementary Figure 4: Synaptic transmission is fully rescued in cultured CAPS DKO neurons expressing CADPS-1 ${ }^{14821}$. (A) Mean amplitude of AP-evoked EPSCS ( $n=27,25$ and 15 cells, of which 5 DKO cells showed no AP-evoked responses, Kruskal-Wallis test, Dunn's Multiple Comparison Test, $\left.{ }^{* * *}<<0.001\right)$. (B) RRP size determined by sucrose solution application ( $n=26$ and 23 cells). (C) Mean vesicular release probability calculated by dividing the charge of AP-evoked EPSCs by the charge of sucrose evoked responses ( $n=26$ and 23 cells). (D) and (E) Amplitude and frequency of spontaneously occurring mEPSCs ( $n=15$ and 14 cells). (F) Paired-pulse ratio during AP trains at the indicated frequencies ( $n=16-25$ cells). (J) Averaged steady-state EPSC responses during trains of APs at indicated frequencies ( $n=16-25$ cells). (Bars in plots depict mean and SEM. $N=4$ cultures). 


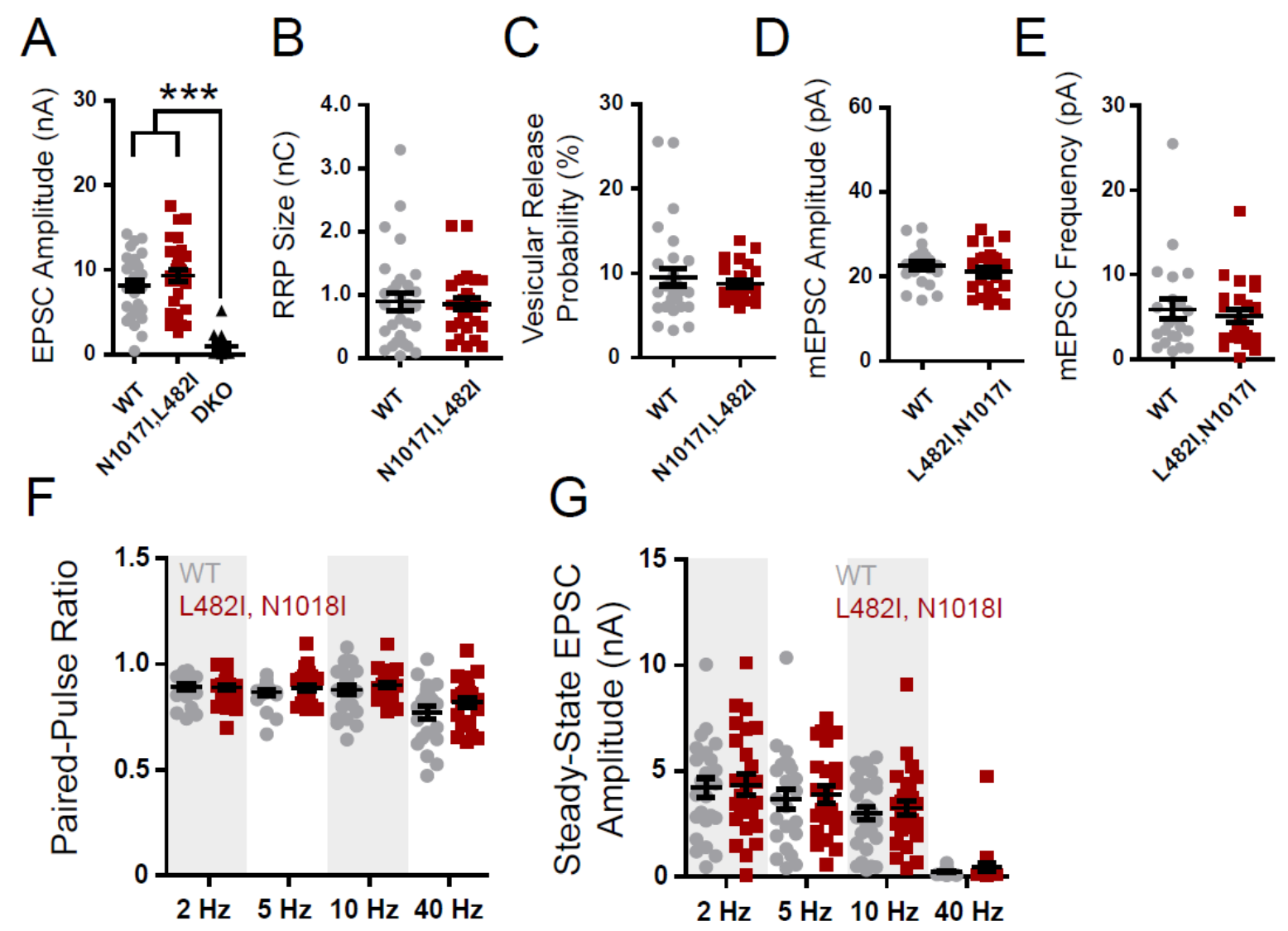

Supplementary Figure 5: Synaptic transmission is fully rescued in cultured CAPS DKO neurons expressing CADPS-14821, ${ }^{N 10171}$. (A) Mean amplitude of AP-triggered EPSCS ( $n=31,30$ and 16 cells, of which 1 DKO cell showed no AP-evoked responses, Kruskal-Wallis test, Dunn's Multiple Comparison Test, $\left.{ }^{* * * P}<0.001\right)$. (B) RRP size determined by sucrose solution application ( $n=30$ and 28 cells). (C) Mean vesicular release probability calculated by dividing the charge of AP-evoked EPSCs by the charge of sucrose evoked responses ( $n=30$ and 28 cells). (D) and (E) Amplitude and frequency of spontaneously occurring mEPSCs ( $n=22$ and 24 cells). (F) Paired-pulse ratio during AP trains at the indicated frequencies ( $n=23-28$ cells). (J) Averaged steady-state EPSC responses during trains of APs at indicated frequencies ( $n=23-28$ cells). (Bars in plots depict mean and SEM. $N=4$ cultures). 

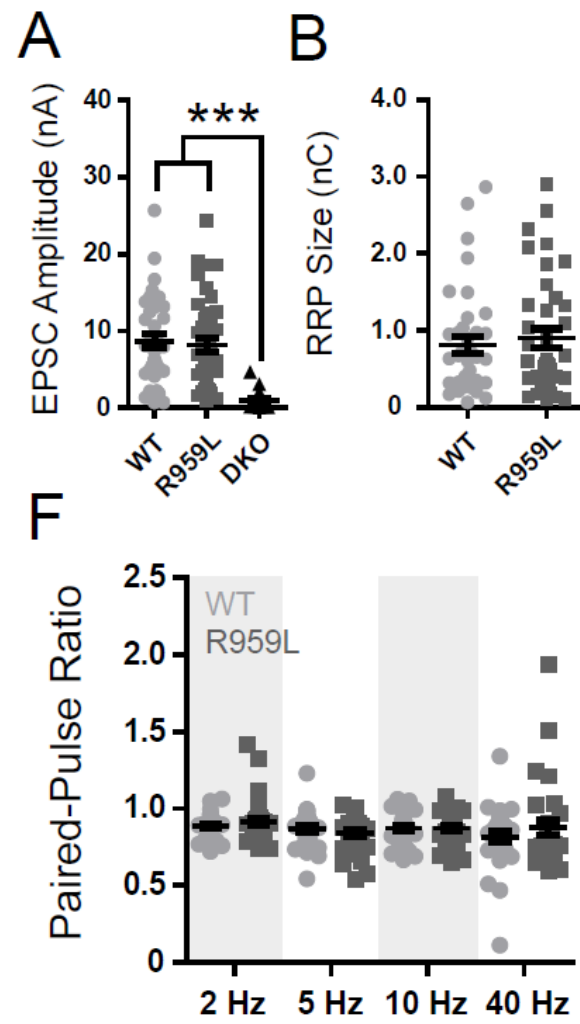

C

$\mathrm{D}$

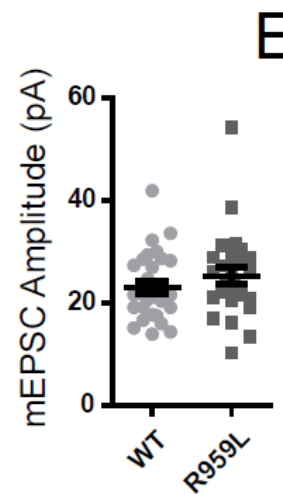

G

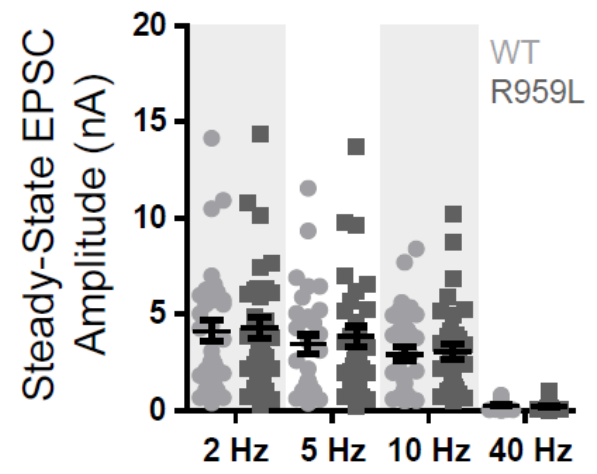

Supplementary Figure 6: Synaptic transmission is fully rescued in cultured CAPS DKO neurons expressing CADPS-1 ${ }^{\text {R959L. }}$. (A) Mean amplitude of AP-triggered EPSCS ( $n=39,42$ and 25 cells, of which 6 DKO cells showed no AP-evoked responses, Kruskal-Wallis test, Dunn's Multiple Comparison Test, $\left.{ }^{* * *}<0.001\right)$. (B) RRP size determined by sucrose solution application ( $n=37$ and 39 cells). (C) Mean vesicular release probability calculated by dividing the charge of AP-evoked EPSCs by the charge of sucrose evoked responses ( $n=37$ and 39 cells). (D) and (E) Amplitude and frequency of spontaneously occurring mEPSCS ( $n=30$ and 26 cells). (F) Paired-pulse ratio during AP trains at the indicated frequencies ( $n=30-36$ cells). (J) Averaged steady-state EPSC responses during trains of APs at indicated frequencies ( $n=30-36$ cells). (Bars in plots depict mean and SEM. $N=6$ cultures). 


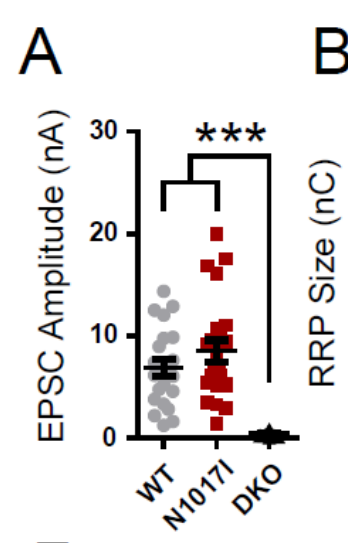

$\mathrm{F}$

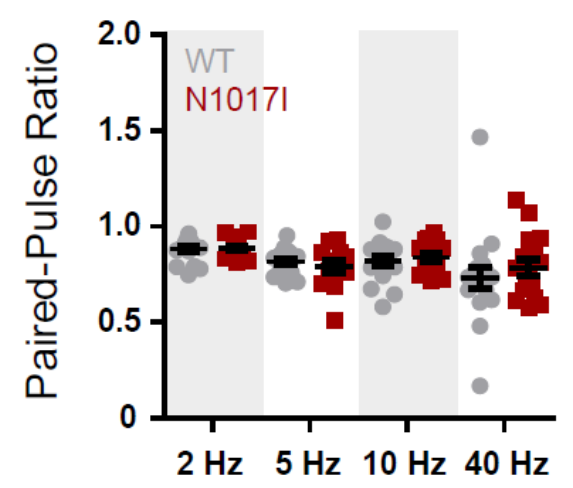

C

D

$E$
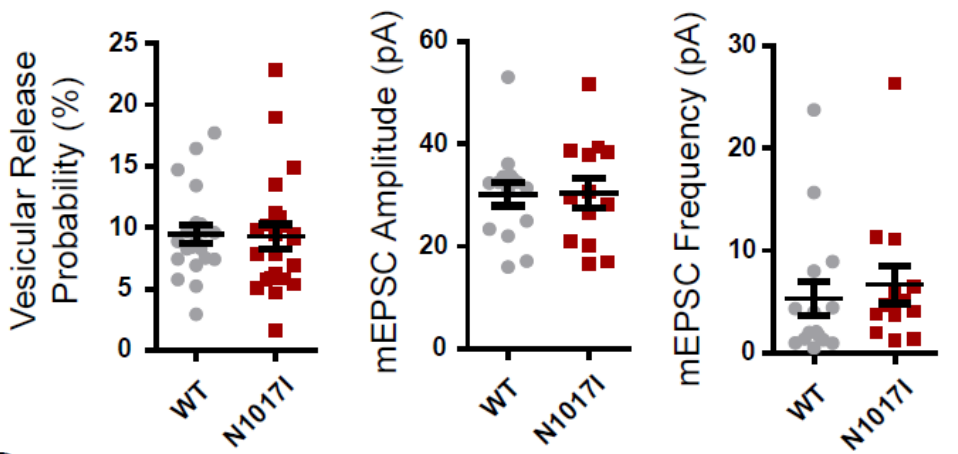

G

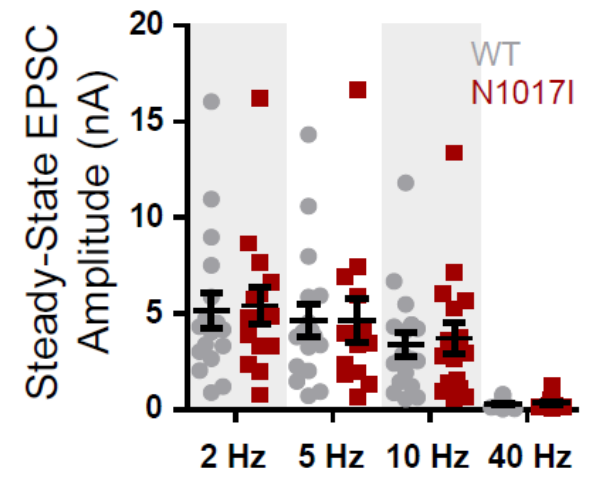

Supplementary Figure 7: Synaptic transmission is fully rescued in cultured CAPS DKO neurons expressing CADPS$1^{\mathrm{N} 10171}$. (A) Mean amplitude of AP-triggered EPSCs ( $n=23,23$ and 17 cells, of which 2 DKO cells showed no APevoked responses, One-Way ANOVA, Bonferroni's Multiple Comparison test, ${ }^{* *} P<0.001$ ). (B) RRP size determined by sucrose solution application ( $n=22$ and 23 cells). (C) Mean vesicular release probability calculated by dividing the charge of AP-evoked EPSCS by the charge of sucrose evoked responses ( $n=22$ and 23 cells). (D) and (E) Amplitude and frequency of spontaneously occurring mEPSCs ( $n=15$ and 13 cells). (F) Paired-pulse ratio during AP trains at the indicated frequencies ( $n=13-19$ cells). (J) Averaged steady-state EPSC responses during trains of APS at indicated frequencies ( $n=13-19$ cells). (Bars in plots depict mean and SEM. $N=4$ cultures). 
Supplementary table 1: Brief summary of the genetic status of mice that were born after CRISPR targeting.

\begin{tabular}{|l|l|}
\hline Number of animals & Genetic status \\
\hline 11 & No genomic modifications detected \\
\hline 5 & Mosaic for frame shift mutations caused by NHEJ \\
\hline 1 & Mosaic for exon2 deletion and deletion of R157,F158 \\
\hline 3 & No information available \\
\hline
\end{tabular}
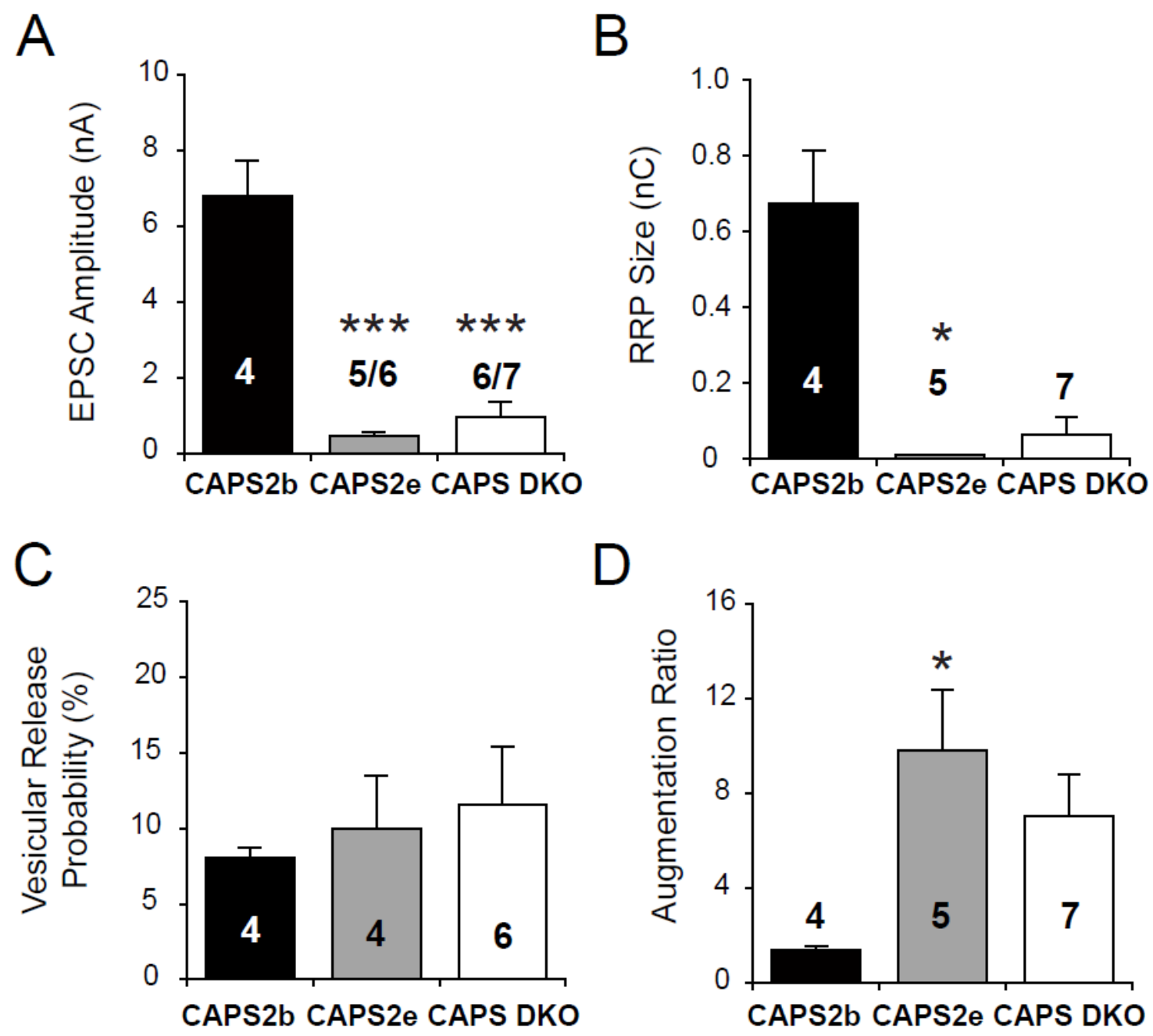

\footnotetext{
Supplementary Figure 8: CAPS2e does not rescue transmitter release in cultured CAPS DKO when expressed by lenti viruses (A) Mean amplitude of AP-triggered EPSCs, (One-Way ANOVA, Bonferroni's Multiple Comparison test, ***P $<0.001$ ). (B) RRP size determined by sucrose solution application (Kruskal-Wallis test, Dunn's Multiple Comparison Test, ${ }^{*} P<0.05$ (). (C) Mean vesicular release probability calculated by dividing the charge of AP-evoked EPSCS by the charge of sucrose evoked responses.(D) Augmentation ratio between the EPSC response $2 \mathrm{~s}$ after 100 AP pulses at $40 \mathrm{~Hz}$ and the first EPSC response during the AP train (One-Way ANOVA, Bonferroni's Multiple Comparison test, ***P $<0.001$ ). (The inset in bars indicates number of cells. Bars in plots depict mean and SEM. $N=1$ culture)
} 

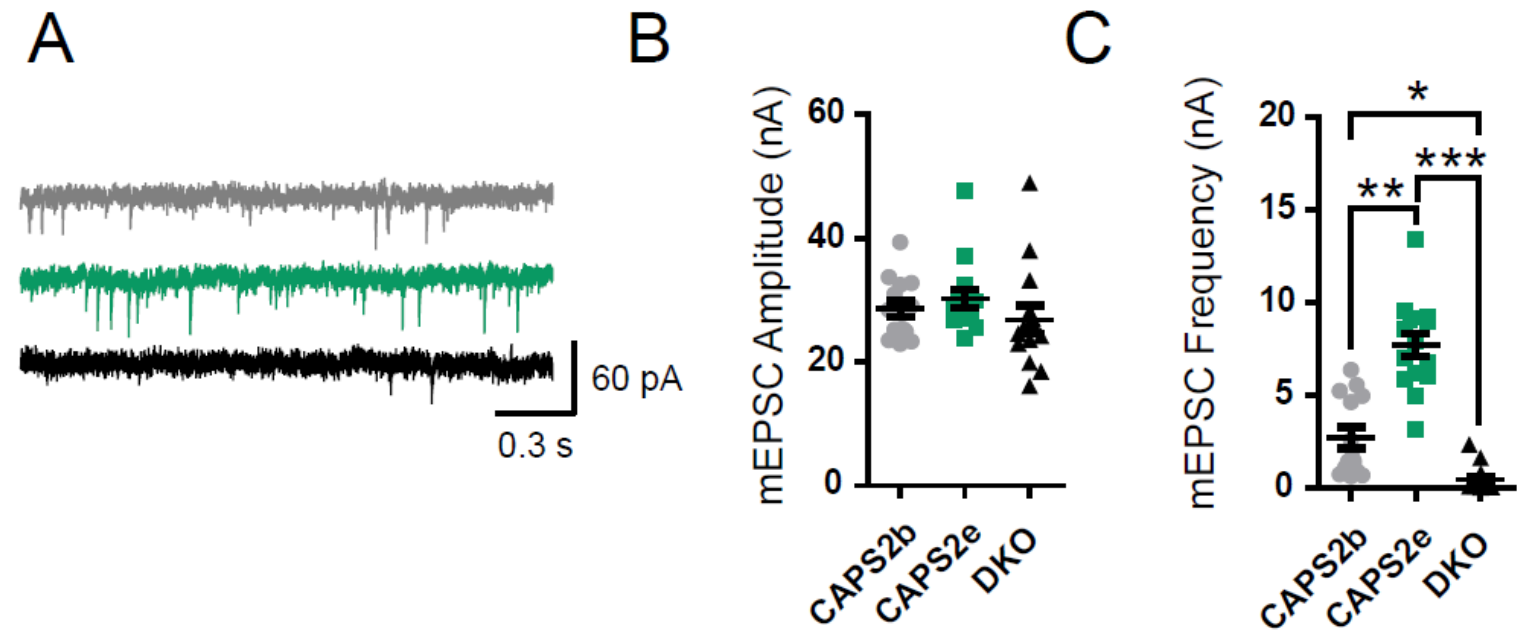

Supplementary Figure 9: The MUN domain of CAPSs may be required to stabilize the primed state of SVs. (A) Representative recordings of spontaneously occurring mEPSCS (B) and (C) Mean amplitude and frequency of mEPSCs measured in presence of 300 nM TTX for 100s ( $n=14,15$ and 14 cells, Kruskal-Wallis test, Dunn's Multiple Comparison Test, ${ }^{*} P<0.05,{ }^{*} p<0.01,{ }^{* *} P<0.001$ ). (Bars in plots depict mean and SEM. $N=3$ cultures).

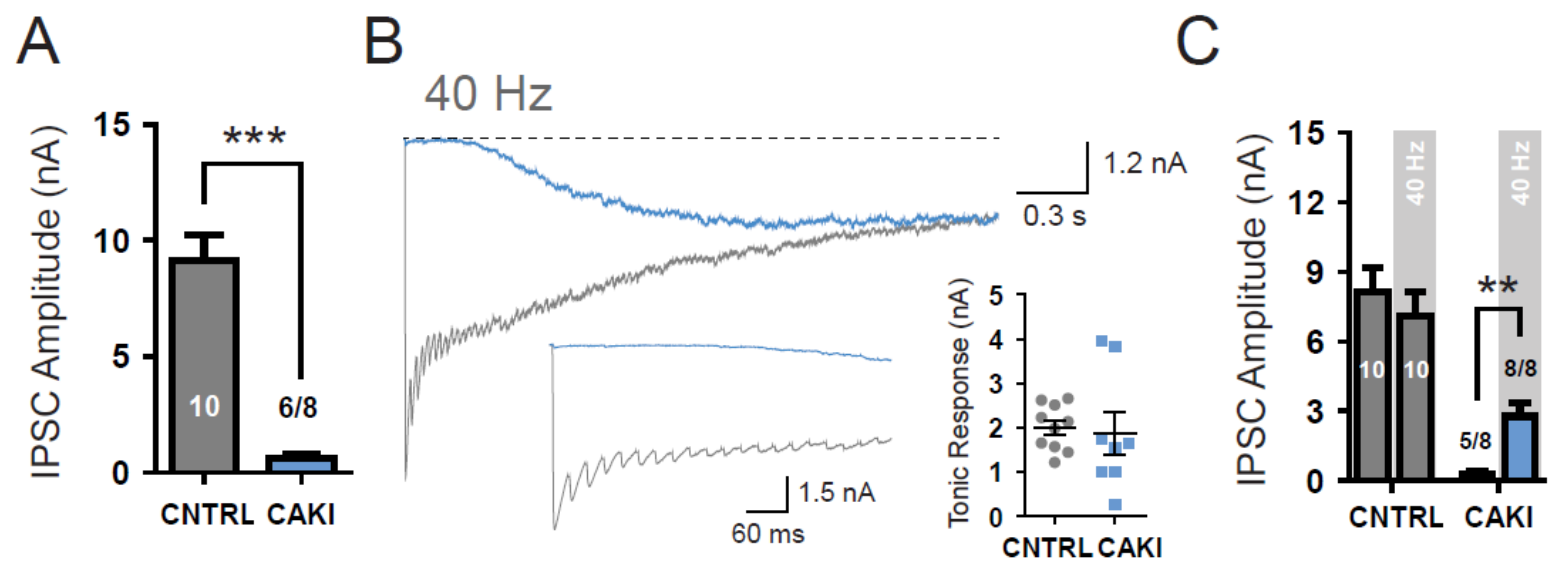

Supplementary Figure 10: Basic electrophysiological characterization of cultured inhibitory neurons from the hippocampus of CAKI mice. (A) Mean amplitude of AP-evoked IPSCS measured in control-and CAKI neurons, respectively ( $n=10$ and 8 cells, of which 2 CAKI cells did not show IPSCs and were excluded from analysis, Student's t-test, $P<0.001)$ (B) Averaged EPSC responses during a train of $100 \mathrm{APs}$ at $40 \mathrm{~Hz}$. The inset depicts a magnification of the first 20 EPSCS and tonic responses used for quantification were measured during the last $0.5 \mathrm{~s}$ of the stimulus ( $n=10$ and 8 cells). (C) EPSC amplitude before and after $40 \mathrm{~Hz}$ AP trains ( $n=10$ and 8 cells, Student's $t$-test $P<0.01$ ). (Bars in plots depict mean and SEM. N = 3 cultures). 


\section{CURRICULUM VITAE}

\section{EDUCATION 04/2013 - present}

PhD studies

Max Planck Institute for Experimental Medicine

Department of Molecular Neurobiology (Dr. Nils Brose)

09/2011-03/2013

Master studies (M.Sc.)

International Max Planck Research School for

Neurosciences, University of Göttingen, Germany

10/2008 - 09/2011

Undergraduate studies in Biology (B.Sc.)

University of Bremen, Germany

\section{RESEARCH EXPERIENCES}

\section{Graduate Studies (2012-present)}

Max Planck Institute of Experimental Medicine

(supervision by Drs. JeongSeop Rhee and Nils Brose), Thesis Committee

Members: Erwin Neher, Reinhard Jahn

\section{Summer school and courses}

ELSC International School for "In-vivo Intracellular Recordings" Eilat, Israel (07/2013-08/2013)

Course on Matlab in Biopsychology and Neuroscience, German Primate Center, Göttingen (05/14 - 06/14)

Visiting scientist, Scanziani lab, UCSF, USA

(07/2016-08/2016)

Undergraduate Studies (2011)

University of Bremen, Centre for Biomolecular Interactions, Dr. Sörge Kelm Title of thesis „Myelin-Associated Glycoprotein induces the Expression of Cytokines in Astrocytes"

\section{SCHOLARSHIPS/ AWARDS}

\section{4-2016}

Boehringer Ingelheim Fonds PhD Fellowship (highly competitive stipend with a success rate of $7 \%$ )

Awarded travel grants by the Boehringer Ingelheim Fonds:

$113^{\text {th }}$ International Titisee Conference "Building Tools for quantifying brain and behavior", participation by invitation only (04/2016)

Gordon Research Conference on "Modulation of Neural Circuits and Behavior" Hong Kong, China, poster presentation (08/2015)

Gordon Research Seminar and Conference on "Synaptic Transmission" Waterville Valley, USA, poster presentation (08/2014) 
PUBLICATIONS

\section{TEACHING}

Nguyen Truong CQ*, Nestvogel D*, Ratai O, Schirra C, Stevens DR, Brose N, Rhee J, Rettig J. 2014. Secretory vesicle priming by CAPS is independent of its SNARE-binding MUN domain. Cell Reports 9:902-909.

*Co-first author

Kawabe, H., Mitkovski, M., Kaeser, P.S., Hirrlinger, J., Opazo, F., Nestvogel, D., Kalla, S., Fejtova, A., Verrier, S.E., Bungers, S.R., et al. (2017). ELKS1 localizes the synaptic vesicle priming protein bMunc13-2 to a specific subset of active zones. J Cell Biol 216, 1143-1161

\section{5-2016}

Tutor for lectures on neurodevelopment, synaptic function and techniques in electrophysiology

2015-2016

Supervisor of two master students

2012-2015

Teaching assistant for laboratory courses in patch clamp physiology

$01 / 2011-02 / 2011$

Teaching assistant for 1st year undergraduate students carrying out a basic methods course in cell biology

\section{SERVICE AND LEADERSHIP}

$11 / 2013-05 / 2016$

Representative for PhD students of the International Max

Planck Research School for Neuroscience, Göttingen

\section{5/2013 \& 05/2015}

Member of organizing committees for the $5^{\text {th }}$ and $6^{\text {th }}$ biennial Neuroscience Conference "Neurizons", Göttingen

09/2014

Member of organizing committee for a joint retreat between PhD students of the Helmholtz International Research School "Molecular Neurobiology", Berlin and the International Max Planck Research School for Neuroscience, Göttingen

$11 / 2012$

Organizer of a public, introductory course for patch clamp recordings in cultured cells at a local science event ("Nacht des Wissens", Göttingen)

$10 / 2011-10 / 2012$

Representative for Master's students and member of the program committee of the Max Planck Research School for Neuroscience, Göttingen 